FHWA/IN/JTRP-2008/26

Final Report

VALIDATION OF NCAT STRUCTURAL TEST TRACK EXPERIMENT USING INDOT APT FACILITY

\author{
Eyal Levenberg \\ Rebecca S. McDaniel
}

September 2009 
Final Report

FHWA/IN/JTRP-2008/26

\title{
VALIDATION OF NCAT STRUCTURAL TEST TRACK EQUIPMENT USING INDOT APT FACILITY
}

\author{
By \\ Eyal Levenberg \\ Postdoctoral Researcher \\ Rebecca S. McDaniel \\ Technical Director \\ and \\ Jan Olek \\ Professor of Civil Engineering and Director \\ North Central Superpave Center \\ School of Civil Engineering \\ Purdue University \\ Joint Transportation Research Program \\ Project No. C-36-31R \\ File No. 2-11-18 \\ SPR-2813 \\ Conducted in Cooperation with the \\ Indiana Department of Transportation and the \\ Federal Highway Administration \\ U.S. Department of Transportation
}

The contents of this report reflect the views of the authors who are responsible for the facts and accuracy of the data presented herein. The contents do not necessarily reflect the official views or policies of the Indiana Department of Transportation and Federal Highway Administration. This report does not constitute a standard, specification, or regulation.

Purdue University

West Lafayette, Indiana

September 2009 
TECHNICAL REPORT STANDARD TITLE PAGE

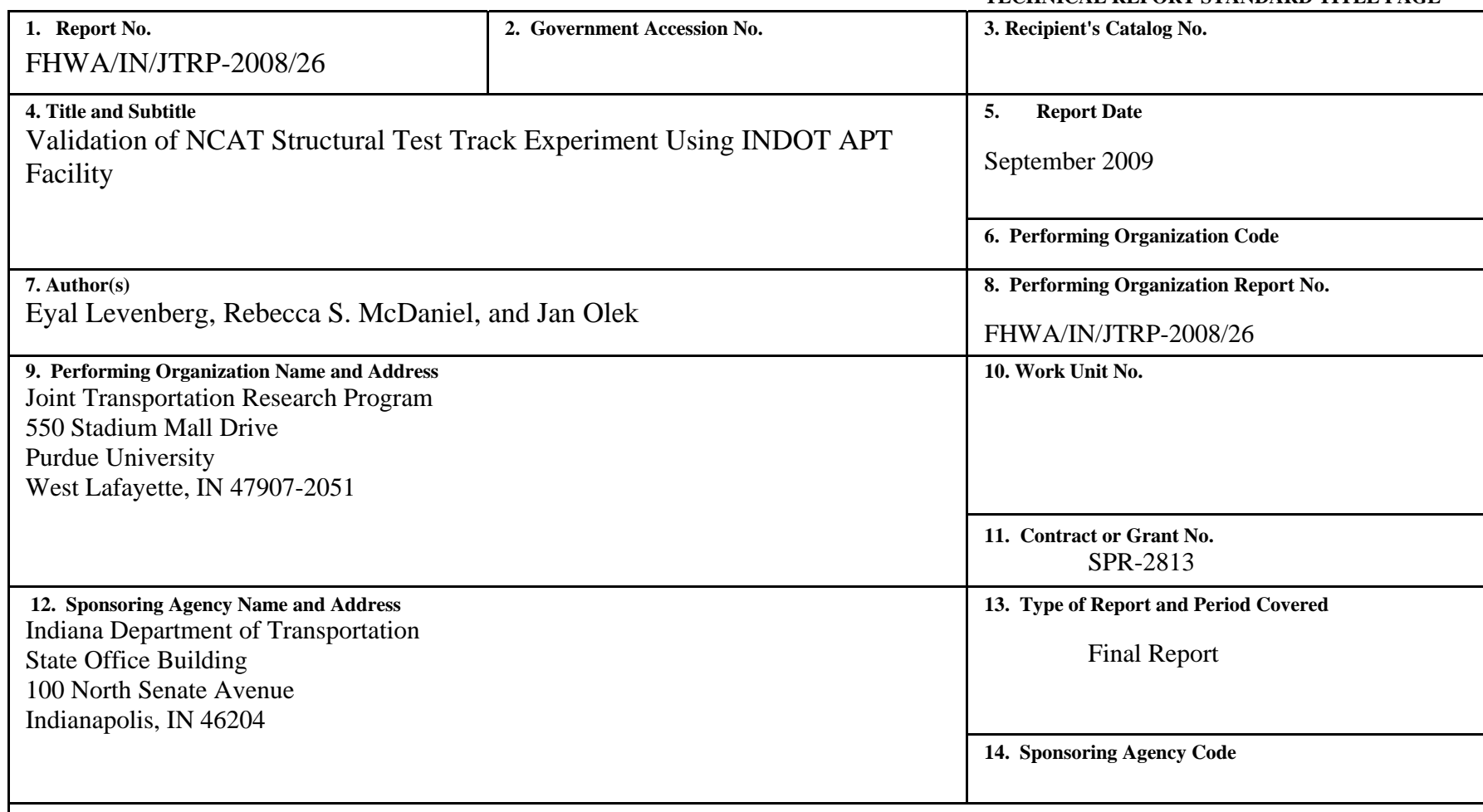

15. Supplementary Notes

Prepared in cooperation with the Indiana Department of Transportation and Federal Highway Administration.

16. Abstract

The National Center for Asphalt Technology (NCAT) operates a full-scale test road for studying the response and performance of asphalt pavements. During the 2003 - 2005 testing phase, NCAT instrumented eight of their test sections with stress and strain gauges. Two of the test sections were later replicated, along with embedded instrumentation, for subsequent testing in the accelerated pavement testing (APT) facility operated by the Indiana Department of Transportation. The availability of similarly constructed and instrumented pavement systems loaded in different conditions offered a unique opportunity to develop and test the forecastability of pavement models. Exploring this aspect is the topic of the present work, in which an attempt is made to use the APT experiment in conjunction with laboratory test results, and forecast resilient responses obtained at NCAT that were generated under completely different loading and environmental conditions. The modeling and analysis methodologies are outlined in detail and the calculation results are compared with NCAT measurements. Findings are discussed and recommendations for future research are given. .

17. Key Words

asphalt pavements, accelerated pavement testing, embedded instrumentation, resilient response, forecastability, inverse analysis, elasticity, viscoelasticity, transverse isotropy.
18. Distribution Statement

No restrictions. This document is available to the public through the National Technical Information Service, Springfield, VA 22161

\section{Security Classif. (of this report) Unclassified}

\section{Security Classif. (of this page)}

Unclassified

21. No. of Pages
173

22. Price 


\section{ACKNOWLEDGMENTS}

The principal author is thankful to various individuals for their assistance and involvement during the completion of the report. First, thanks are due to the members of the Study Advisory Committee (SAC): Tommy Nantung, Dave Andrewski, and Kurt Sommer from the Indiana Department of Transportation; Gerald Huber from Heritage Research; Lee Gallivan from the Federal Highway Administration (FHWA) Indiana Division; Lloyd Bandy from the Asphalt Pavement Association of Indiana; John Haddock from Purdue University; and Dudley Bonte from Rieth-Riley Construction.

A sincere appreciation goes to Robert "Buzz" Powell, manager of the NCAT Test Track at Opelika, Alabama, and to David H. Timm from Auburn University for their collaboration and cooperation in developing the instrumentation installation procedures and for sharing the response data recorded at the Track.

Special thanks are owed to Tom Robertson and Calvin Reck from Purdue University for their collaboration in all aspects of the APT experiment starting with construction and instrumentation, and ending with loading and data collection.

I would like to take this opportunity and also thank Terhi Pellinen, the original Principal Investigator (PI) of this project along with her research assistants Jose Llenín and Greg Webster who jointly designed and overlooked the APT experiments and left me with well organized notes and photographs to work with.

Last but not least, and on a more personal level, would like to thank the people of the North Central Superpave Center (NCSC), especially: Jan Olek, Becky McDaniel, and Ayesha Shah, for inviting me to join the NCSC 'family', for giving me the opportunity to work on this project, and for placing their trust in me to see it through to completion. 


\section{TABLE OF CONTENTS}

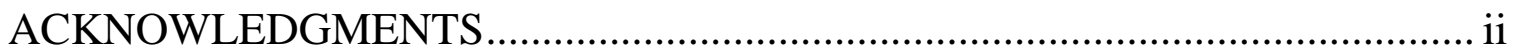

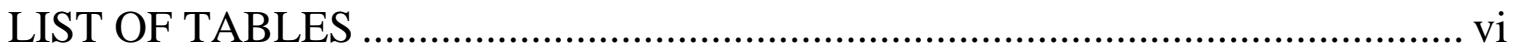

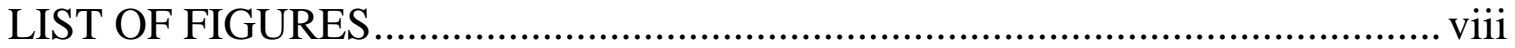

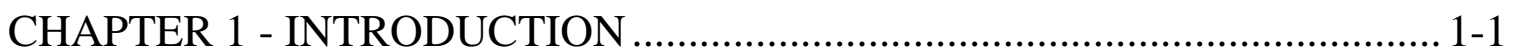

1.1 BACKGROUND AND MOTIVATION..................................................................... 1-1

1.2 PROBLEM STATEMENT, STUDY OBJECTIVES AND SCOPE ……………………..... 1-2

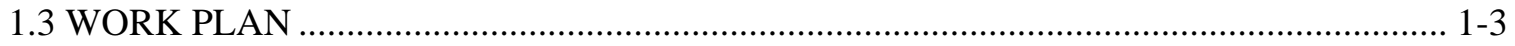

1.4 STRUCTURE OF THE REPORT ……………………………………………….... 1-4

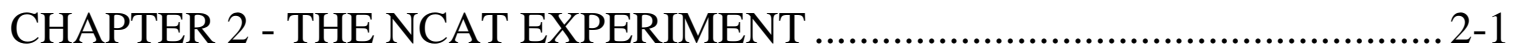

2.1 FACILITY DESCRIPTION.................................................................................... 2-1

2.2 CONSTRUCTION OF TEST SECTIONS ……………………………………….... 2-3

2.2.1 Pavement Structures.................................................................................. 2-3

2.2.2 Subgrade........................................................................................... 2-4

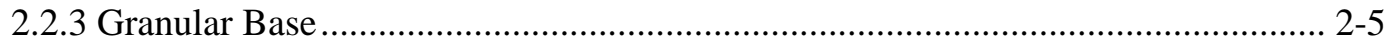

2.2.4 Hot Mix Asphalt.......................................................................................... 2-6

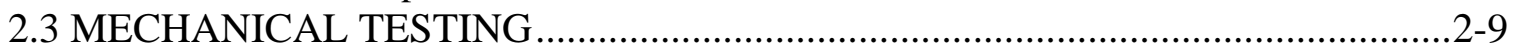

2.3.1 Resilient Modulus of Subgrade and Base Materials.............................................. 2-9

2.3.2 HMA Complex Modulus.................................................................................... 2-12

2.3.3 Falling Weight Deflections ....................................................................... 2-18

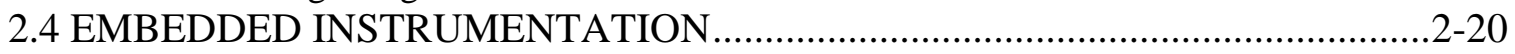

2.4.1 Environmental Monitoring ……………………………………………….... 2-20

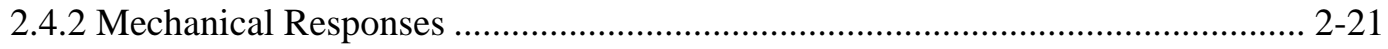

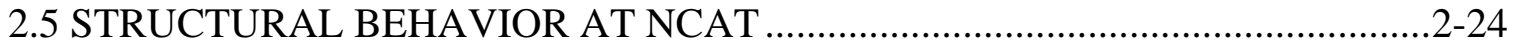

2.5.1 Resilient Response .................................................................................... 2-24

2.5.2 Cracking and Rutting Performance ……………………………………………. 2-30

CHAPTER 3 - THE APT EXPERIMENT .................................................... 3-1

3.1 FACILITY DESCRIPTION ……………………………………………………... 3-1

3.2 COMPOSITION OF TEST SECTIONS .................................................................... 3-5

3.3 MECHANICAL TESTING AND PRELIMINARY ANALYSIS ………………………….. 3-7

3.3.1 Resilient Modulus of Unbound Materials ............................................................. 3-7

3.3.2 HMA Complex Modulus.................................................................................... 3-10

3.3.3 Falling Weight Deflections ………………………………………………... 3-16

3.4 LOADING HISTORY AND ANALYSIS DATASET ...................................................... 3-17

3.4.1 Application of Load Passes .............................................................................. 3-17

3.4.2 Identification of Dataset for Structural Investigation ............................................. 3-21

3.5 STRUCTURAL BEHAVIOR ........................................................................... 3-22

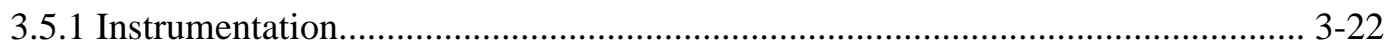

3.5.2 Resilient Response ......................................................................................... 3-25

3.5.3 Rutting and Cracking Performance ...................................................................... 3-29

3.6 DIRECT COMPARISON WITH NCAT RESULTS ............................................................ 3-38 
CHAPTER 4 - BASIC MECHANISTIC ANALYSIS ..................................... 4-1

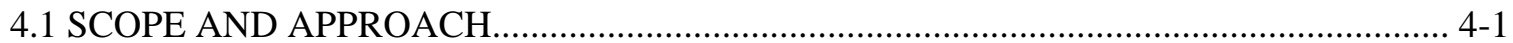

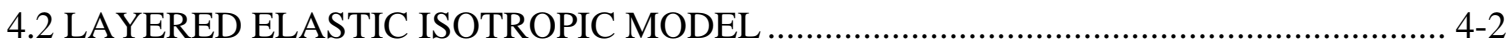

4.2.1 Theory and Computational Implementation.......................................................... 4-2

4.2.2 Calibration to APT Conditions........................................................................... 4-7

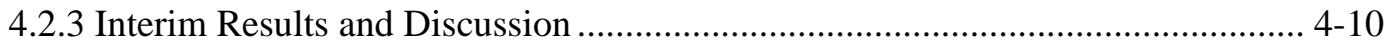

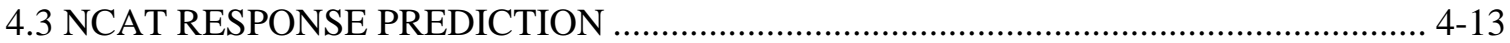

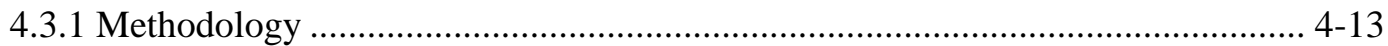

4.3.2 Falling Weight Deflections .............................................................................. 4-17

4.3.3 Traffic Induced Stresses and Strains ............................................................... 4-19

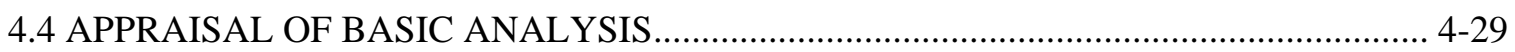

CHAPTER 5 - ADVANCED MECHANISTIC METHODS ............................. 5-1

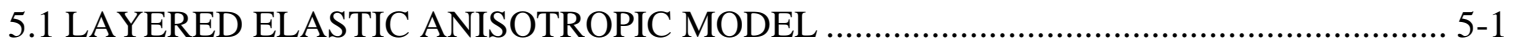

5.1.1 Theory and Computational Implementation...................................................... 5-1

5.1.2 Calibration to APT Conditions ……................................................................. 5-9

5.1.3 NCAT Response Prediction .............................................................................. 5-12

5.2 LAYERED VISCOELASTIC ISOTROPIC MODEL ................................................... 5-18

5.2.1 Theory and Computational Implementation...................................................... 5-18

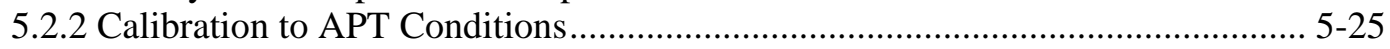

5.2.3 NCAT Response Prediction ............................................................................ 5-30

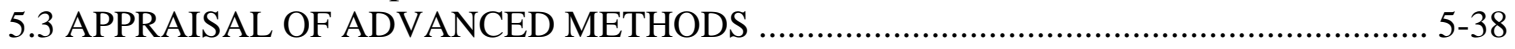

CHAPTER 6 - CONCLUSION ............................................................. 6-1

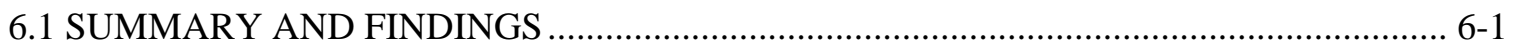

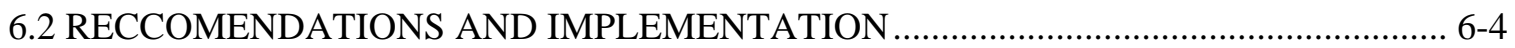

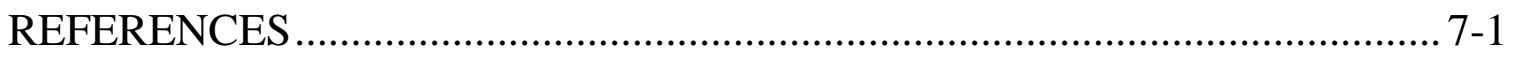

APPENDICES (DVD Available Upon Request) ......................................... 8-1

APPENDIX A: RAW EXPERIMENTAL DATA …................................................. 8-1

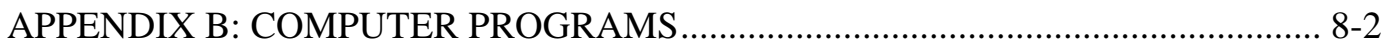

APPENDIX C: REPORTS AND CORRESPONDANCE ......................................... 8-2 


\section{LIST OF TABLES}

Table 2.1.1: Breakdown of axle properties for an 'average' NCAT truck (Priest and Timm, 2006)

Table 2.2.1: HMA design parameters for mixes 1 to 4 (Timm and Priest, 2006) $2-8$

Table 2.3.1: Resilient modulus of subgrade soil with average compaction level of $96 \%$. Raw test results from laboratory reports $2-10$

Table 2.3.2: Resilient modulus of aggregate base with average compaction level of 93\%. Raw test results from laboratory reports

Table 2.3.3: Average complex modulus test results for Mix 1 (Barde and Cardone, 2004).

Table 2.3.4: Average complex modulus test results for Mix 2 (Barde and Cardone, 2004).

Table 2.3.5: Average complex modulus test results for Mix 3 (Barde and Cardone, 2004).

Table 2.3.6: Average complex modulus test results for Mix 4 (Barde and Cardone, 2004).

Table 2.3.7: FWD deflections at N1 section (location according to NCAT database: station 2 inside the wheel path)

Table 2.3.8: FWD deflections at N2 section (location according to NCAT database: station 2 inside the wheel path)

Table 2.5.1: Temperature profile in Section N1 for analysis of resilient response data $2-24$

Table 2.5.2: Tabulated progression of N1 and N2 rutting levels vs. number of applied ESALs.

Table 3.3.1: Resilient modulus of unbound materials (calibrated equation 3.3.2 parameters)

Table 3.3.2: Complex modulus analysis results for a reference temperature of $15.5^{\circ} \mathrm{C}$ based on the approach in Levenberg and Shah (2008)

Table 3.3.3: Peak FWD deflections measured in the center of sections $\mathrm{n} 1$ and $\mathrm{n} 2$

Table 3.4.1: APT pass application log for sections $n 1$ and n2 (original structure) 3-18

Table 3.4.2: APT pass application log for Section n2 (rehabilitated structure) 3-19

Table 3.4.3: APT pass application log for Section $n 1$ (rehabilitated structure) $3-20$

Table 3.5.1: Location of APT instrumentation in Section n1 (relate to Figure 3.5.1) 3-25

Table 4.2.1: Backcalculated layer moduli for pass \#5,000 and pass \#80,000 . 4-10

Table 4.3.1: Combined complex modulus properties for APT n1 / NCAT N1 (based on equations 4.3 .1 to 4.3 .4 )

Table 4.3.2: Matching errors between isotropic LET predictions and NCAT measured responses. Errors are in percent after normalization using the corresponding peak to peak response shown in brackets (in microstrains) 
Table 5.1.1: Backcalculated anisotropic layer moduli for pass \#5,000 ..................................... 5-10

Table 5.1.2: Adjusted anisotropic HMA moduli for FWD response prediction ....................... 5-13

Table 5.2.1: Backcalculated material properties for the layered viscoelastic model

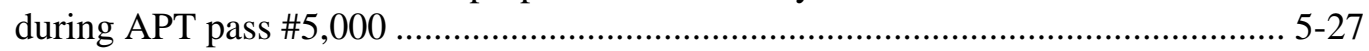

Table 5.2.2: Relative improvement in response predictions for the isotropic LVT compared to the isotropic LET case given in Table 4.3.2 ........................................ 5-32 


\section{LIST OF FIGURES}

Figure 2.1.1: Schematic layout of the 46 test sections at NCAT (Phase II) experiment.............. 2-2

Figure 2.1.2: Photograph of a typical NCAT truck (Priest and Timm, 2006)............................. 2-3

Figure 2.2.1: Structural layers for sections N1 to N8 (Priest and Timm, 2006).......................... 2-4

Figure 2.2.2: Final gradation of upper subgrade soil at N1 and N2 sections (Timm and

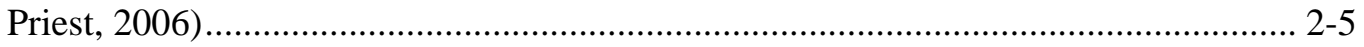

Figure 2.2.3: Gradation of base material at N1 and N2 sections (Timm and Priest,

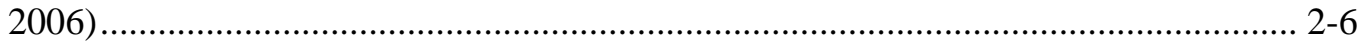

Figure 2.2.4: Sub-layering of HMA in test sections N1 to N8 (Timm and Priest, 2006)............. 2-7

Figure 2.2.5: Design gradation of different mix types used in the NCAT 'structural study' (Timm and Priest, 2006) ................................................................................. 2-8

Figure 2.4.1: Photograph and dimensions (in inches) of an asphalt strain gauge (Timm

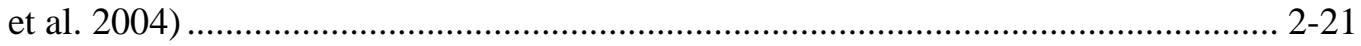

Figure 2.4.2: Photograph of Geokon Earth Pressure cell Model 3500..................................... 2-22

Figure 2.4.3: Sensor layout for section N1 (based on Timm et al., 2004)................................. 2-23

Figure 2.5.1: Vertical stresses (i.e. stress in Z) on top of the base course (upper chart) and on top of the subgrade (lower chart) as a result of one truck pass. Gauges positioned along the Y-axis in Figure 2.4.3.

Figure 2.5.2: Horizontal strains in the loading direction (i.e., strain in Y) at the bottom of the HMA course as a result of one truck pass. Gauges positioned along the Y-axis in Figure 2.4.3

Figure 2.5.3: Horizontal strains in the loading direction (i.e., strain in Y) at the bottom of the HMA course as a result of one truck pass. Gauges offset by $24 \mathrm{in}$. (610 mm) compared to the $\mathrm{Y}$-axis in Figure 2.4.3

Figure 2.5.4: Horizontal strains in the transverse direction (i.e., strain in X) at the bottom of the HMA course as a result of one truck pass. Gauges positioned along Y-axis in Figure 2.4.3.

Figure 2.5.5: Fatigued sections N1 (left photo) and N2 (right photo)...................................... 2-30

Figure 2.5.6: Graphical progression of N1 and N2 rutting levels vs. number of applied ESALs at the Track. 2-32

Figure 3.1.1: Schematic floor plan of INDOT APT facility....................................................... 3-2

Figure 3.1.2: Picture of empty test pit and APT loading system................................................. 3-3

Figure 3.2.1: Composition of APT test pavements $n 1$ and $n 2$.................................................... 3-5

Figure 3.3.1: Resilient modulus of unbound materials - a cross plot of calibrated equation 3.3.2 values and test data 3-10

Figure 3.3.2: Mix 1 dynamic modulus and phase angle master curves @ $15.5^{\circ} \mathrm{C}$...................... 3-13

Figure 3.3.3: Mix 2 dynamic modulus and phase angle master curves @ $15.5^{\circ} \mathrm{C}$..................... 3-14

Figure 3.3.4: Mix 3 dynamic modulus and phase angle master curves @ $15.5^{\circ} \mathrm{C}$...................... 3-14 
Figure 3.3.5: Mix 4 dynamic modulus and phase angle master curves @ $15.5^{\circ} \mathrm{C}$.

Figure 3.3.6: Superimposed dynamic modulus master curves @ $15.5^{\circ} \mathrm{C}$ for mixes 1 to

Figure 3.3.7: Superimposed phase angle master curves @ $15.5^{\circ} \mathrm{C}$ for mixes 1 to 4

Figure 3.3.8: Peak FWD deflections measured in the center of sections n1 and n2

Figure 3.5.1: Plan of embedded instrumentation in APT lane 1 (Section n1).

Figure 3.5.2: Measured vertical stresses in Section $n 1$ on top of the base and on top of the subgrade during pass \#5,000 (solid line) and pass \#80,000 (dashed line)

Figure 3.5.3 Measured horizontal strains at the bottom of the HMA in the direction of loading during pass \#5,000 (solid line) and pass \#80,000 (dashed line)

Figure 3.5.4: Measured horizontal strains at the bottom of the HMA in the transverse direction to the loading during pass \#5,000 (solid line) and pass \#80,000 (dashed line)

Figure 3.5.5: Rutting development in Section $\mathrm{n} 1$ at the central cross section during the first 90,000 load passes (applied without wheel wander)

Figure 3.5.6: Contour plot of Section n1 rutting after 100 passes.

Figure 3.5.7: Contour plot of Section n1 rutting after 500 passes.

Figure 3.5.8: Contour plot of Section $n 1$ rutting after 1,000 passes

Figure 3.5.9: Contour plot of Section $n 1$ rutting after 5,000 passes 3-33

Figure 3.5.10: Contour plot of Section n1 rutting after 10,000 passes. 3-34

Figure 3.5.11: Contour plot of Section n1 rutting after 20,000 passes. 3-34

Figure 3.5.12: Contour plot of Section n1 rutting after 30,000 passes. 3-35

Figure 3.5.13: Contour plot of Section n1 rutting after 40,000 passes. 3-35

Figure 3.5.14: Contour plot of Section n1 rutting after 50,000 passes. 3-36

Figure 3.5.15: Contour plot of Section n1 rutting after 60,000 passes. 3-36

Figure 3.5.16: Contour plot of Section n1 rutting after 70,000 passes. 3-37

Figure 3.5.17: Contour plot of Section n1 rutting after 80,000 passes. 3-37

Figure 3.5.18: Contour plot of Section n1 rutting after 90,000 passes. 3-38

Figure 4.2.1: User interface of the isotropic LET program ELLEA1 (see Appendix B) 4-6

Figure 4.2.2: Resilient responses during APT pass \#5,000. Both measured (solid markers) and model generated (solid line) are shown

Figure 4.2.3: Resilient responses during APT pass \#80,000. Both measured (solid markers) and model generated (solid line) are shown

Figure 4.3.1: Combined HMA dynamic modulus and phase angle master curves for a reference temperature of $15.5^{\circ} \mathrm{C}$ (based on Table 4.3.1) 4-15

Figure 4.3.2: Combined HMA time-temperature shifting for a reference temperature of $15.5^{\circ} \mathrm{C}$ (based on Table 4.3.1) 4-17

Figure 4.3.3: Measured and projected peak FWD deflections at NCAT N1. 4-18 
Figure 4.3.4: Layout of N1 gauge array (refer to Figure 2.4.3) and travel path positioning of the center point of the rightmost truck tire (connecting arrows)

Figure 4.3.5: Travel paths of center of rightmost truck wheels over the N1 gauge array at NCAT for the different axles in Table 2.1.1.

Figure 4.3.6: Calculated and measured N1 responses - right side of steering axle (1S)

Figure 4.3.7: Calculated and measured N1 responses - right side of drive axle (1D and 2D)

Figure 4.3.8: Calculated and measured N1 responses - right side of first trailer axle (1T)

Figure 4.3.9: Calculated and measured N1 responses - right side of third trailer axle (3T)

Figure 4.3.10: Calculated and measured N1 responses - right side of last trailer axle (5T) $4-28$

Figure 5.1.1: User interface of the anisotropic LET program ELLEA2 $5-8$

Figure 5.1.2: ELLEA2 display of property and algorithm restriction for the example shown in Figure 5.1.1

Figure 5.1.3: Comparison of measured resilient responses in the APT during pass \#5,000 with responses computed using the anisotropic layered model (isotropic case is reproduced from Figure 4.2.2)

Figure 5.1.4: Comparison of measured peak FWD deflections at NCAT N1 with projected peak deflections using anisotropic LET (isotropic case reproduced from Figure 4.3.3).

Figure 5.1.5: Comparison of anisotropic LET projections with measured N1 responses - right side of steering axle (1S). Isotropic case reproduced from Figure 4.3.6

Figure 5.1.6: Comparison of anisotropic LET projections with measured N1 responses - right side of third trailer axle (3T). Isotropic case reproduced from Figure 4.3.9

Figure 5.2.1: Scheme for simulating a moving load on a layered viscoelastic model $5-22$

Figure 5.2.2: Indicial admittance of a layered viscoelastic system (example). Strain response due to a unit intensity 'input' of an APT half-axle passing along four offset distances from the evaluation point

Figure 5.2.3: Comparison of backcalculated relaxation modulus with that interconverted from complex modulus test results

Figure 5.2.4: Comparison of measured resilient responses in the APT during pass \#5,000 with responses computed using the isotropic viscoelastic layered model

Figure 5.2.5: Comparison of isotropic LVT projections with measured N1 responses right side of steer axle (1S). Isotropic case reproduced from Figure 4.3.6. 
Figure 5.2.6: Comparison of isotropic LVT projections with measured N1 responses right side of drive axle (1D and 2D). Isotropic case reproduced from Figure 4.3.7

Figure 5.2.7: Comparison of isotropic LVT projections with measured N1 responses right side of first trailer axle (1T). Isotropic case reproduced from Figure 4.3.8

Figure 5.2.8: Comparison of isotropic LVT projections with measured N1 responses right side of third trailer axle (3T). Isotropic case reproduced from Figure 4.3.9

Figure 5.2.9: Comparison of isotropic LVT projections with measured N1 responses right side of fifth (last) trailer axle (5T). Isotropic case reproduced from Figure 4.3.10 


\section{CHAPTER 1 - INTRODUCTION}

\subsection{BACKGROUND AND MOTIVATION}

Despite years of systematic research the design and analysis of asphalt pavements still includes dominant empirical components. This state of affairs may be ascribed to the complexity of the problem: first, the mechanical behavior of pavement materials and subgrades is not well understood and cannot always be sufficiently controlled; second, material properties continuously change during the pavement service life due to natural processes such as oxidation and age hardening; third, only rough estimates can be provided for basic design inputs such as traffic loads and environmental conditions; and finally, the notion of structural failure is not defined in a clear cut manner. For these reasons available analysis methods are heavily based on past experience and as such cannot be used reliably with non-traditional materials and components and cannot aid in optimizing pavement designs.

Accelerated pavement testing (APT) facilities were built in an effort to address some of the aforementioned limitations. These facilities offer controlled study conditions in which pavements and subgrades of known materials can be loaded and closely monitored. Traditionally, the design and use of these facilities was driven by empirical approaches, resulting in studies in which the performance of different pavements under similar loading conditions was monitored and compared. In these tests significant effort was placed on accelerating rutting and cracking damage in an attempt to capture in a relatively short period of time (of the order of months) equivalent field experience that can only be gained over a period of many years. More recent APT studies have been driven by pavement mechanics principles in an effort to accommodate or aid in the development of more rational design methods. In either case it is widely accepted that experimental results obtained in APT facilities are not directly applicable to the field and that the sophisticated interpretation is required.

Pavetrack is a full scale test road located near the campus of Auburn University in Alabama, operated and managed by the National Center for Asphalt Technology (NCAT); it is a closed-loop facility that applies accelerated truck traffic to 46 adjoining 
experimental sections paved with Hot Mix Asphalt (HMA). The 2003 - 2005 testing phase at NCAT, also known as Phase II, included the construction, loading and continuous monitoring of eight different instrumented pavement structures, referred to as sections N1 to N8. The primary objective of this so-called 'structural study' was to provide high quality data for validating the Mechanistic-Empirical Pavement Design Guide (MEPDG) (ARA Inc., 2004). For this purpose, during the two year loading period, performance data (e.g., cracking, rutting, roughness and skid resistance) and response data (i.e., stresses, strains and deflections) were recorded within the structure and subgrade along with prevailing environmental conditions (e.g., temperatures and moisture levels).

In 2004, the Indiana Department of Transportation (INDOT) and Purdue University engaged in a smaller-scale research project that is closely related to the NCAT 'structural study' experiment. In this project, the two NCAT test sections N1 and N2 were replicated in the INDOT APT facility along with embedded instrumentation. These sections, referred to herein as $n 1$ and $n 2$, were loaded in the APT over a two year period between 2004 and 2006.

Consequently, similar instrumented pavement structures were made available, loaded in completely different conditions with a closely monitored environment. It is the overall motivation of this study to try and establish a relation between the behavior (i.e., both response and performance) of the pavements in the APT facility and their corresponding behavior at the NCAT test track. By establishing such a relation, the methodology used can be potentially applied with confidence to future APT studies as means of forecasting field behavior of replicate pavement systems.

\subsection{PROBLEM STATEMENT, STUDY OBJECTIVES AND SCOPE}

By their very nature, the testing conditions in the APT facility are considerably more uniform compared to field conditions. For example, in the current APT study use was made of a single axle configuration, single axle weight and one loading speed; also, the entire experiment was carried out under constant temperature conditions. Accordingly, the observed pavement behavior in the APT is the result of these limited conditions 
only. Therefore, the problem is how to interpret APT experimental results such that they could be applied to different environmental and loading conditions.

The main objective of this study is to devise and validate an analysis scheme by which experimental data collected in the APT experiment can be used to successfully forecast the corresponding pavement behavior at the NCAT test track. The analysis scheme is based on mechanistic principals in order to provide a rational basis for the interpretation and allow the incorporation of different complexity levels depending on the desired accuracy of the outcome. The work includes an underlying basic assumption that the pavement structures in both cases were similar. It is beyond the scope of this research to consider the case of different structures.

Focus is placed on the analysis of responses, i.e., stresses, strains and deflections, and less on cracking and rutting performance. This is mainly because accurate response prediction is the underlying key for reliable performance forecasting and due to a scarcity of adequate performance data from both experiments. Moreover, it is important to note that only resilient (recoverable) responses will be addressed. This is mainly because the type of embedded instrumentation installed in both experiments was only suited for monitoring dynamic (transient) pavement reactions and not for recording permanent (irrecoverable) responses. In fact, this latter point is a known shortcoming of all available strain and stress gauges commercially available at this time.

This study has also a secondary objective which is to summarize the work performed and document the available experimental data. A clear description of what was done, how it was done and what data was collected may encourage additional studies using the existing records.

\subsection{WORK PLAN}

The initial work plan, described in a previous report by Llenín and Pellinen (2004), consisted of tasks related to construction of the APT experiment and execution of the accompanying laboratory tests (see Appendix C). At this time test data from both the APT and NCAT experiments are available; therefore, the work plan outlined herein 
includes only the tasks required to achieve the aforementioned study objectives. The scope and purpose of each task are described hereafter.

Task 1 consists of careful study, systematic documentation and presentation of pertinent test data from both the APT and NCAT experiments. The aim here is to familiarize the reader with relevant details of the work done. It is mostly descriptive in nature with limited pre-processing of the data. The raw test results are contained in Appendix A.

Task 2 includes the identification of response data suitable for mechanistic analysis; the aim here is to identify a subset of the available data that is most suitable and sufficient for accomplishing the main study objective.

Task 3 contains the development and calibration of a mechanistic pavement model based on APT data only. This task is the central element of the entire methodology. As a basic case, the mechanistic 'engine' of the MEPDG is applied, i.e., layered elastic theory (LET) with isotropic material properties. Also considered are two more advanced models, namely: LET with transversely isotropic material properties and layered viscoelastic theory (LVT) with linear isotropic material properties. In each case

the numerical values of the model parameters are obtained from inverse analysis by simulating the APT experiment and matching the measured responses.

In Task 4 the capabilities of the APT model are enhanced. For this purpose an analysis scheme is developed by which the derived material properties obtained in Task 3 are adjusted in order for them to apply to other loading configurations, other loading speeds, and different environmental conditions. These adjustments are based primarily on the analysis of laboratory test data.

Finally, Task 5 deals with model validation using NCAT results. For this purpose the loading and environment at NCAT are simulated and the forecastability of the 'enhanced' model (Task 4) is assessed by comparison with NCAT measurements.

\subsection{STRUCTURE OF THE REPORT}

Chapters 2 and 3 address tasks 1 and 2. Chapter 2 is mainly narrative with minimal interpretation; containing relevant information from the NCAT experiment such as description of the facility loading conditions, composition and instrumentation of the 
test sections and recorded field and laboratory behavior. Chapter 3 summarizes the APT work; it includes a description of the loading history and environment prevailing during the experiment, some preliminary analyses of available the test data and identification of dataset most suitable for structural investigation. This chapter also presents the recorded structural behavior for the selected dataset. Thereafter a direct comparison with the NCAT results is provided to emphasize the need for more intricate and fundamental analysis.

Tasks 3, 4 and 5 are addressed by Chapters 4 and 5; both contain the development of mechanistic models for representing the pavement systems considered. Chapter 4 deals only with isotropic LET while Chapter 5 deals anisotropic LET and isotropic LVT. In these chapters it is shown how the necessary material properties are calibrated using the APT experiment. Thereafter, they explain how to apply the models to other loading and environmental conditions that were not included in the calibration. Finally, selected responses at NCAT are predicted using the models and compared with field measurements for validation purposes. The final chapter, Chapter 6, includes a short summary of the entire report outlining the main findings. It also provides some general recommendations and implementation suggestions for INDOT. 


\section{CHAPTER 2 - THE NCAT EXPERIMENT}

This chapter summarizes the 'structural study' experiment conducted by NCAT between the years 2003 and 2005 (phase II). The focus is on sections N1 and N2 which were later replicated in the APT experiment. Reference to the original reports is provided so the reader can trace the source and obtain additional data.

\subsection{FACILITY DESCRIPTION}

The NCAT test track is a 1.7 miles $(2.8 \mathrm{~km})$ oval shaped closed-loop asphalt road located near Opelika, Alabama. The primary objective for building the track was to provide a practical, engineering driven, research tool for validation of laboratory tests and pavement design procedures under accelerated and controlled traffic conditions (Brown et al., 2002). The general track layout is shown in Figure 2.1.1. As can be seen, the facility allows for the simultaneous loading of 46 experimental sections; there are 26 sections on the tangents, each about $200 \mathrm{ft}$ long $(60.96 \mathrm{~m})$. The curved potions of the track host the remaining 20 sections. The track was constructed to have two lanes and paved shoulders on each side of the roadway. Both lanes were designed and constructed to have the same materials and thicknesses within a given test section. One lane was used for traffic and pavement performance monitoring. The other lane provided a safety lane in case of truck breakdowns and construction access for repairing existing sections.

Construction of the track was completed in 2000 and the first cycle of tests (i.e., Phase I) took place between the years 2000 and 2002. The second testing cycle (Phase II), which is relevant to this report, took place between the years 2003 and 2005. Most of the sections from the 2000 experiment were either left as-is for the 2003 experiment or rehabilitated by shallow milling and inlaying. These sections were originally involved in a comparative rutting study which was extended to the second cycle of testing. Only eight new sections were rebuilt from the subgrade up for the 2003 experiment: sections N1 to N8 (Timm et al., 2004; 2006). These sections were devoted to a 'structural study' focusing primarily upon the effects of HMA thickness and binder type/grade on the dynamic pavement responses under truck loading. These pavements were instrumented to monitor load induced horizontal strains in the bottom of the HMA, 
vertical compressive stresses on top of the base and subgrade, moisture in the unbound materials and temperature within the HMA. Additionally, these sections were investigated by periodic deflection testing and monitored for structural distresses by employing routine surface condition surveys. Reportedly this was done to allow for later validation of the MEPDG. The focus herein is on sections N1 and N2, which were the only sections from the 'structural study' replicated in the INDOT APT.

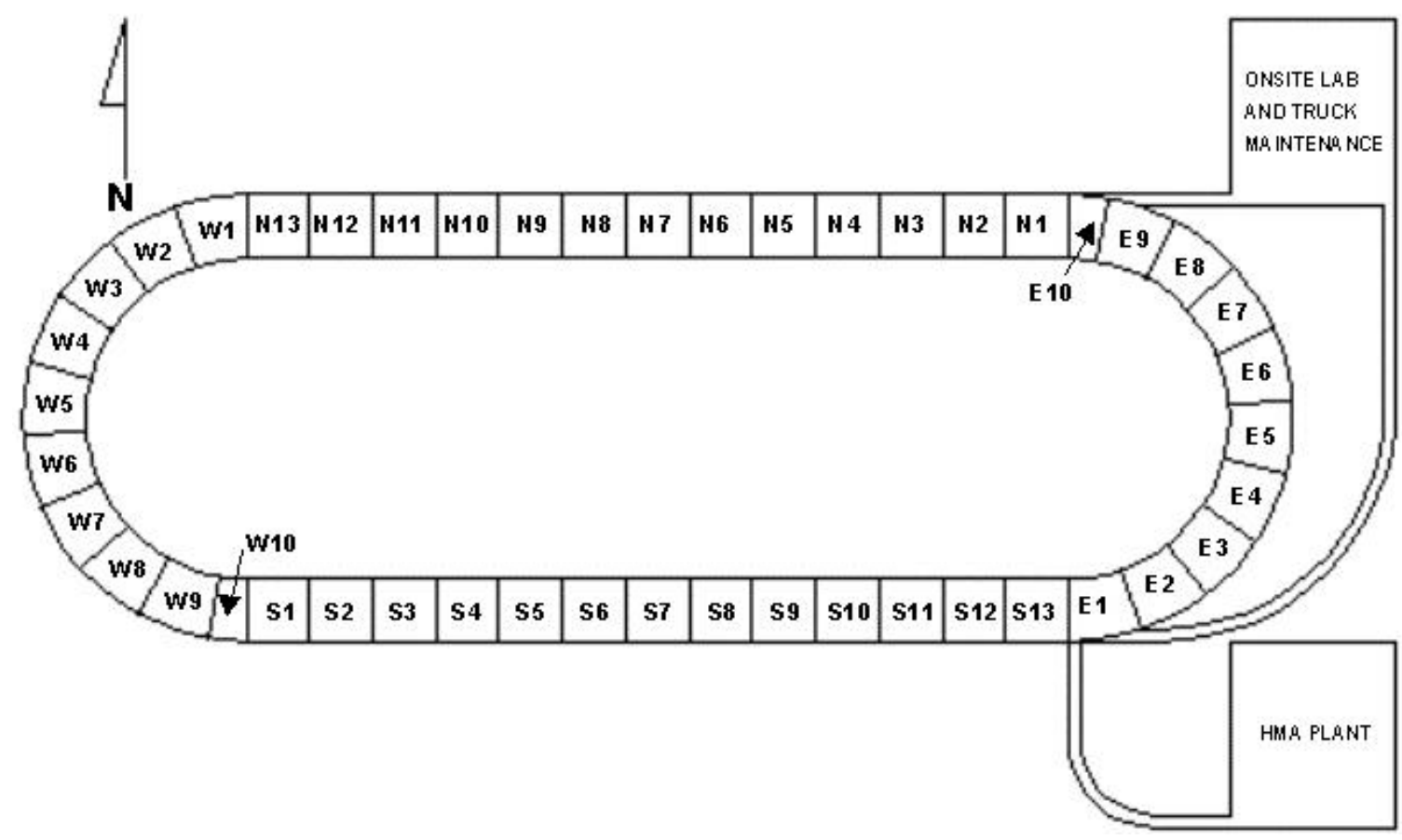

Figure 2.1.1: Schematic layout of the 46 test sections at NCAT (Phase II) experiment.

Traffic loadings at the track are applied using a designated fleet of tractor-trailer trucks (triple trailer), each traveling at $45 \mathrm{mph}$ or $792 \mathrm{in.} / \mathrm{s}(72.4 \mathrm{~km} / \mathrm{h}$ or $20.1 \mathrm{~m} / \mathrm{s})$. Drivers are utilized to operate the trucks, and their operation consists of two 7.5 hour shifts, five days a week. Typically, each truck completes about 26 laps in an hour. A picture of one NCAT truck is provided in Figure 2.1.2. As can be seen, the truck has several wheel assemblies: single-axle single-wheels (steer axle); tandem-axle dualwheels (drive axle); and single-axle dual-wheels (trailer axle). The 'average' weight carried by the individual axles is shown in table 2.1.1. This is an 'average' weight because each of the five trucks in the fleet has a slightly different load. The coefficient of variation (COV) of the loads is also shown in the table with values in the range of 
1.7\% to $4.9 \%$ (average of 3.1\%). Standard tires were used (Priest et al., 2005) identified as $275 / 80 \mathrm{R} 22.5$ and inflated to 100 psi.

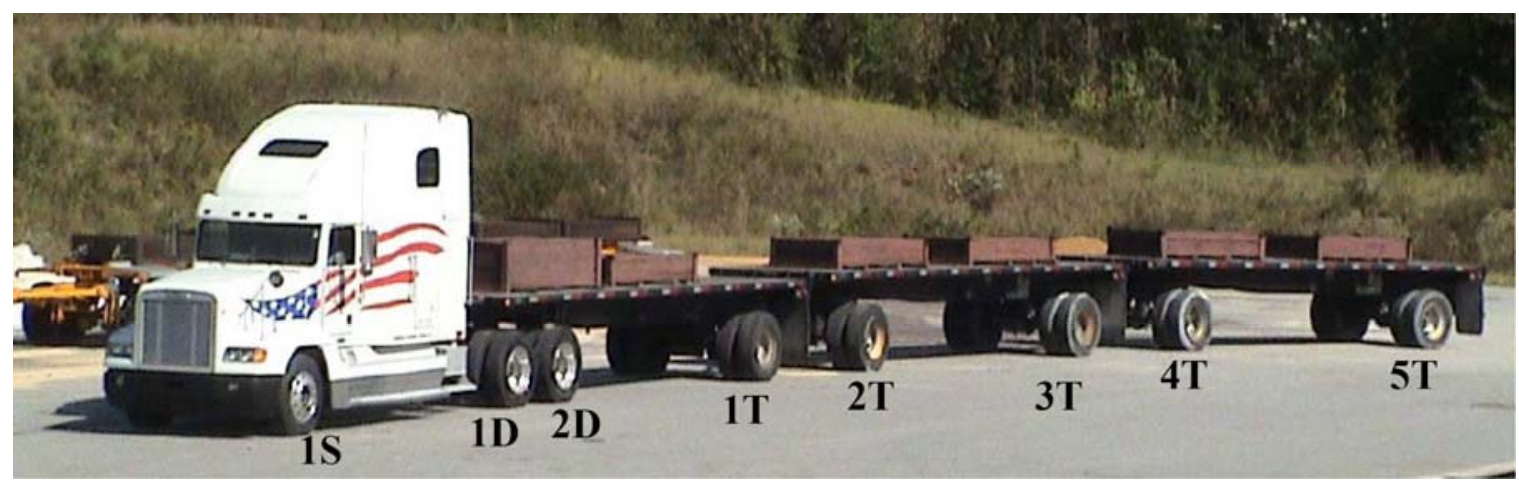

Figure 2.1.2: Photograph of a typical NCAT truck (Priest and Timm, 2006).

Table 2.1.1: Breakdown of axle properties for an 'average' NCAT truck (Priest and Timm, 2006).

\begin{tabular}{|c|c|c|c|c|c|c|c|c|}
\hline Axle-name & Steer & \multicolumn{2}{|c|}{ Drive } & \multicolumn{5}{|c|}{ Trailer } \\
\hline $\begin{array}{c}\text { Axle- } \\
\text { Number }\end{array}$ & $1 \mathrm{~S}$ & $1 \mathrm{D}$ & $2 \mathrm{D}$ & $1 \mathrm{~T}$ & $2 \mathrm{~T}$ & $3 \mathrm{~T}$ & $4 \mathrm{~T}$ & $5 \mathrm{~T}$ \\
\hline Axle-type & $\begin{array}{c}\text { Single- } \\
\text { Axle }\end{array}$ & \multicolumn{2}{|c|}{ Tandem-Axle } & $\begin{array}{c}\text { Single } \\
\text {-Axle }\end{array}$ & $\begin{array}{c}\text { Single- } \\
\text { Axle }\end{array}$ & $\begin{array}{c}\text { Single } \\
\text {-Axle }\end{array}$ & $\begin{array}{c}\text { Single } \\
\text {-Axle }\end{array}$ & $\begin{array}{c}\text { Single } \\
\text {-Axle }\end{array}$ \\
\hline $\begin{array}{c}\text { No. of } \\
\text { Wheels }\end{array}$ & 2 & 4 & 4 & 4 & 4 & 4 & 4 & 4 \\
\hline $\begin{array}{c}\text { Average } \\
\text { Axle-Load, } \\
\text { lb (kg) }\end{array}$ & $\begin{array}{c}10,680 \\
(4,850)\end{array}$ & $\begin{array}{c}20,320 \\
(9,225)\end{array}$ & $\begin{array}{c}20,290 \\
(9,210)\end{array}$ & $\begin{array}{c}21,010 \\
(9,540)\end{array}$ & $\begin{array}{c}20,760 \\
(9,425)\end{array}$ & $\begin{array}{c}21,310 \\
(9,675)\end{array}$ & $\begin{array}{c}20,550 \\
(9,330)\end{array}$ & $\begin{array}{c}20,613 \\
(9,360)\end{array}$ \\
\hline $\begin{array}{c}\text { COV for 5 } \\
\text { Trucks }\end{array}$ & $3.9 \%$ & $3.9 \%$ & $4.9 \%$ & $2.2 \%$ & $2.5 \%$ & $1.7 \%$ & $3.6 \%$ & $2.2 \%$ \\
\hline
\end{tabular}

\subsection{CONSTRUCTION OF TEST SECTIONS}

\subsubsection{Pavement Structures}

Pavement structures in the NCAT 'structural study' were designed using the 1993 AASHTO guide employing three different traffic levels. The final outcome is shown in Figure 2.2.1. It may be seen that each structure includes 6 in. (152 mm) of unbound granular base under HMA layers of varying thickness having different composition and/or binder grade. Both N1 and N2 sections were designed with 5 in. (127 mm) of HMA, sections N3 and N4 were designed with 9 in. (229 mm) of HMA, and sections 
N5 to N8 were designed with 7 in. $(178 \mathrm{~mm})$ of HMA each. It is important to note that these are design values and that the actual as-constructed thicknesses varied slightly.

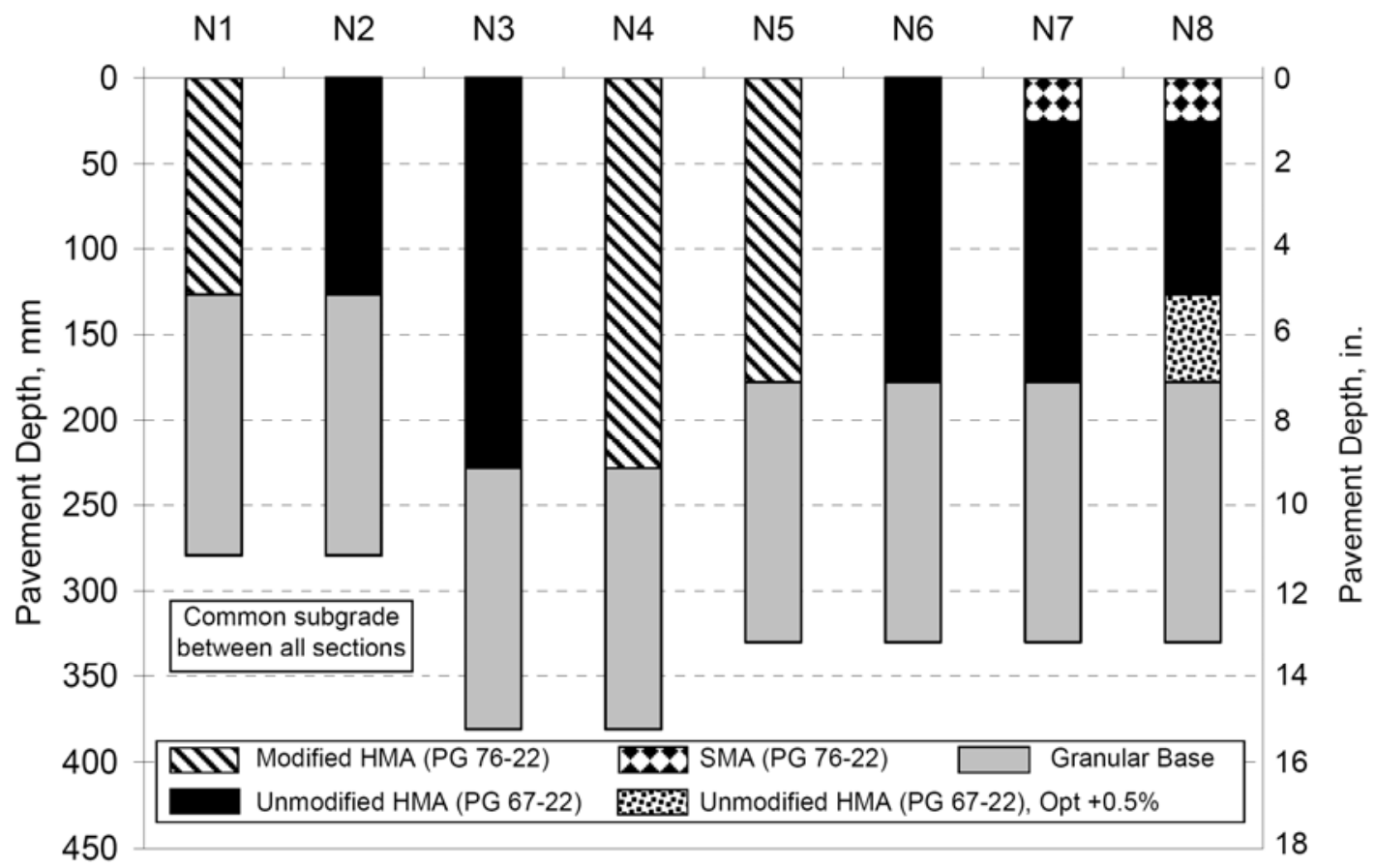

Figure 2.2.1: Structural layers for sections N1 to N8 (Priest and Timm, 2006).

\subsubsection{Subgrade}

The upper subgrade for all eight test sections (N1 to N8) was processed to a depth of 30 in. (762 mm) from the pavement surface (Timm and Priest, 2006). The material was then compacted in layers using vibratory pad-foot rollers (e.g., Dynapac CA15PD). It should be noted that large cobbles, which were originally present in the material, broke down under rolling. The final outcome was classified as an A-4(0) soil; the resulting gradation curve after rolling operations is presented in Figure 2.2.2. As can be seen, 100 percent of the material is smaller than the $1.5 \mathrm{in}$. sieve (38.1 mm opening), and more than 45\% passes the \#200 sieve (0.075 mm opening). Below the upper subgrade the material originating from the 2000 track construction remained untouched.

When the upper subgrade soil was compacted in the lab using modified Proctor effort, the resulting maximum dry unit weight was 119.6 pcf $\left(1918 \mathrm{~kg} / \mathrm{m}^{3}\right)$ with a corresponding moisture content of $8.6 \%$ (i.e., laboratory optimum). The average as-built 
moisture contents for sections N1 and N2 were 10\% and 11\% respectively. The average in-place wet unit weight was $132.0 \mathrm{pcf}\left(2116 \mathrm{~kg} / \mathrm{m}^{3}\right)$ for both sections, and the corresponding dry unit weights were 120.0 and 118.9 pcf (1924 and $\left.1906 \mathrm{~kg} / \mathrm{m}^{3}\right)$. From this information it may be concluded that the relative in-place degree of compaction, based on dry densities, was $100.3 \%$ and $99.4 \%$ for sections N1 and N2 respectively.

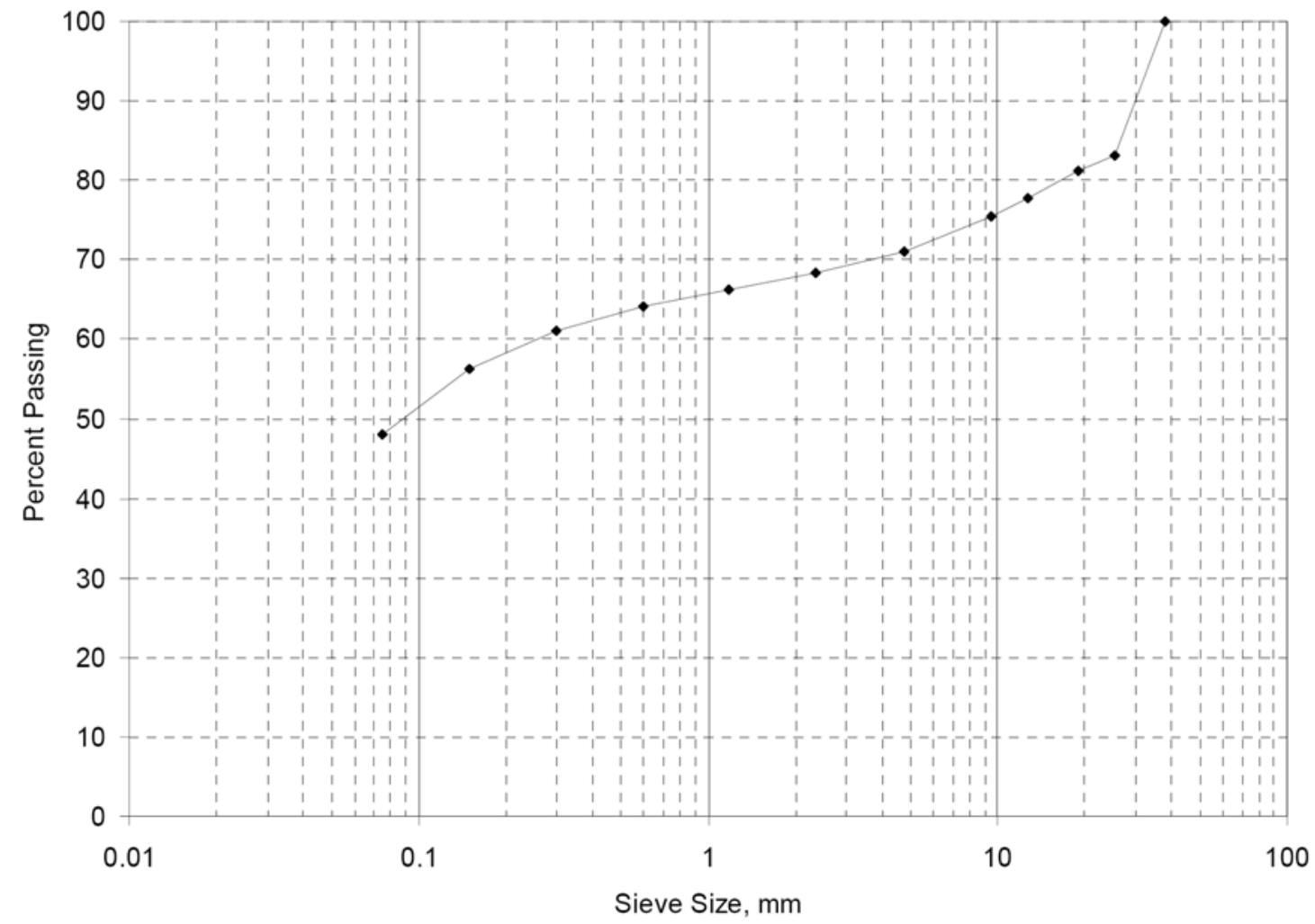

Figure 2.2.2: Final gradation of upper subgrade soil at N1 and N2 sections (Timm and Priest, 2006).

\subsubsection{Granular Base}

The granular base consisted of a well graded crushed granite material, compacted in a single 6 in. (152 mm) layer. The gradation of this material is shown in Figure 2.2.3. As can be seen, 100 percent of the material is smaller than the $1.5 \mathrm{in}$. sieve $(38.1 \mathrm{~mm}$ opening). Also note that less than $10 \%$ of the material is passes the \#200 sieve (0.075 $\mathrm{mm}$ opening). Loose base samples were recompacted in the lab using modified Proctor effort, resulting in a maximum dry unit weight of $137.9 \mathrm{pcf}\left(2211 \mathrm{~kg} / \mathrm{m}^{3}\right)$ and a corresponding moisture content of 9.2\% (i.e., laboratory optimum). The average field 
dry density values were $138.0\left(2213 \mathrm{~kg} / \mathrm{m}^{3}\right)$ for both sections. With respect to dry densities, the relative compaction degree for the base layer in both sections was $100.1 \%$. The as-built moisture content varied slightly: $6.4 \%$ in section $\mathrm{N} 1$ and $6.6 \%$ in section N2.

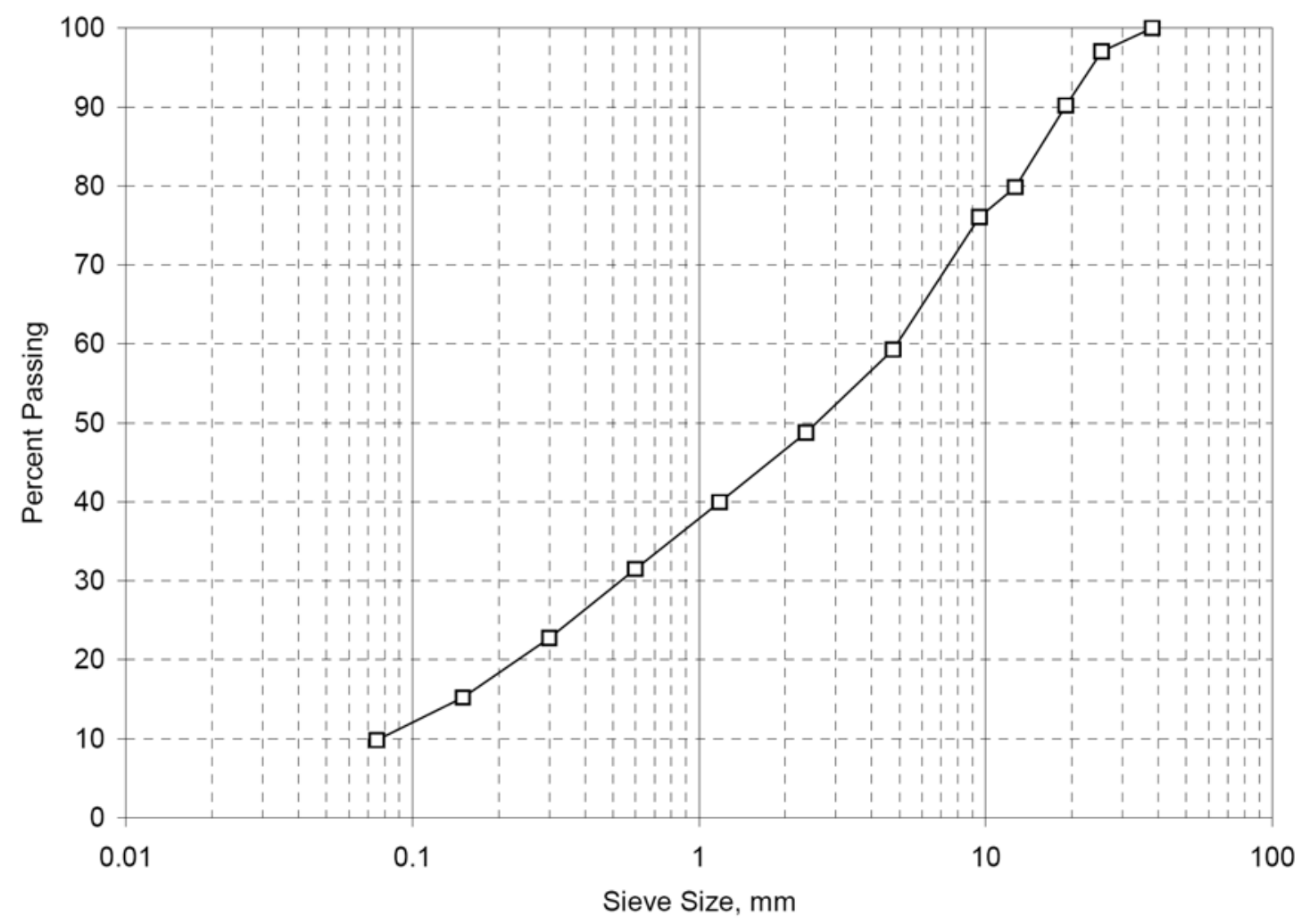

Figure 2.2.3: Gradation of base material at N1 and N2 sections (Timm and Priest, 2006).

\subsubsection{Hot Mix Asphalt}

Figure 2.2.4 presents the sub-layering of the HMA in the different test sections in the 'structural study' (Timm and Priest, 2006). Sections N1 and N2 were paved in three lifts to a total HMA thickness of 5 in. (127 mm). The bottom and intermediate lifts are each 2 in. $(50.8 \mathrm{~mm})$ thick while the top lift is 1.0 in. $(25.4 \mathrm{~mm})$ thick. 


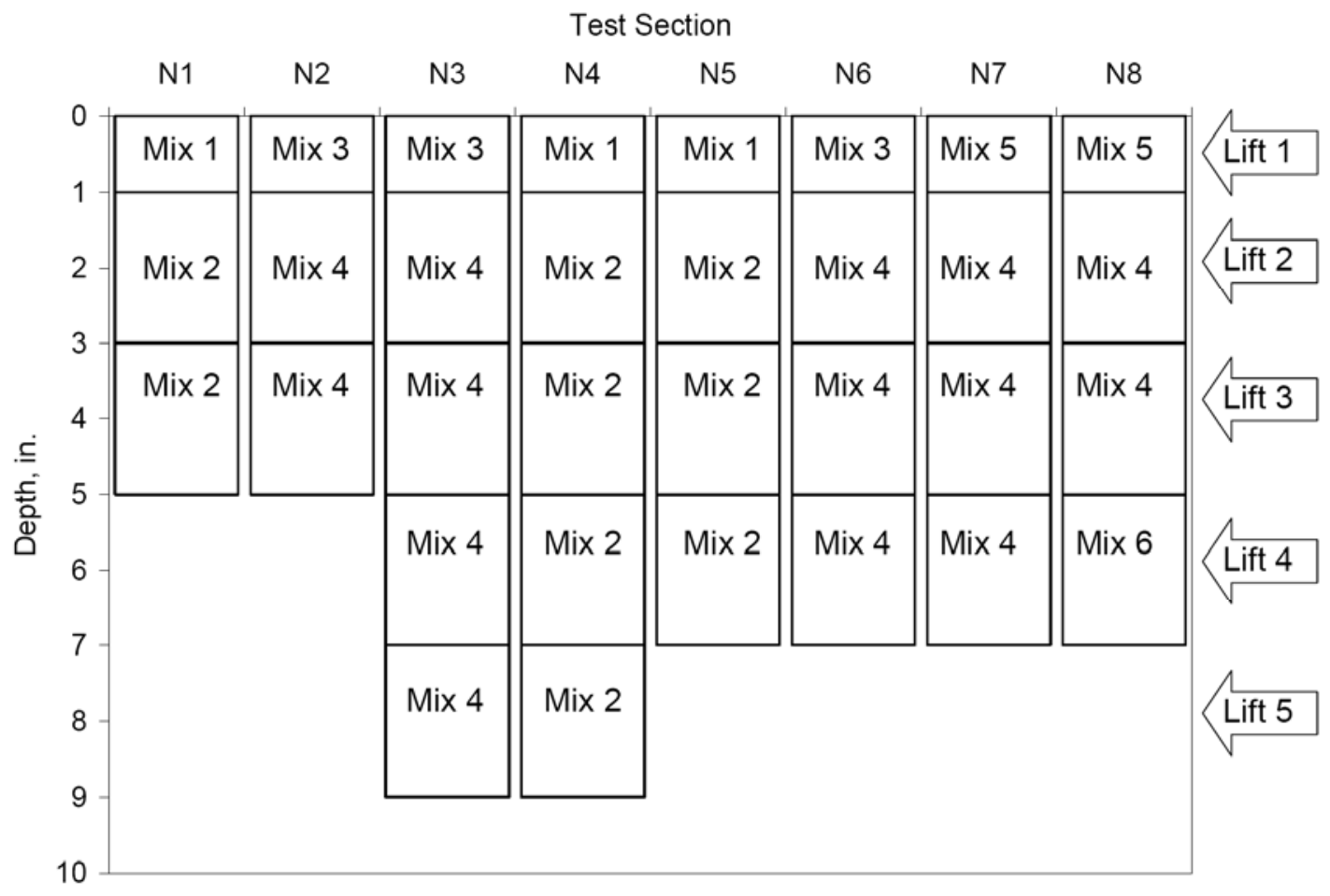

Figure 2.2.4: Sub-layering of HMA in test sections N1 to N8 (Timm and Priest, 2006).

Table 2.2.1 presents the individual mixture design parameters for mixes 1 to 4 which were paved in the N1 and N2 sections. It may be seen that the surface mixes 1 and 3 differ by the type of binder and corresponding preparation temperatures. SBS modified PG 76-22 was used for Mix 1, and unmodified PG 67-22 was used for Mix 3. Both mixes (see Figure 2.2.5) were designed with a 'wearing' (dense) gradation having a nominal maximum aggregate size (NMAS) of $9.5 \mathrm{~mm}$ (3/8 in.) and a compactive effort of 80 gyrations. At the optimum binder content (i.e., 6.13\% effective), the samples had $4.3 \%$ air voids and $17.9 \%$ VMA. Similar to the surface mixes, the intermediate and bottom mixes (mixes 2 and 4) differed by the type of binder. SBS modified PG 76-22 was used for Mix 2, and unmodified PG 67-22 was used for Mix 4. Both mixes (see Figure 2.2.5) were designed with a 'Base' (also dense) gradation having a NMAS of $19 \mathrm{~mm}$ (3/4 in.) and a compactive effort of 80 gyrations. At optimum binder content (4.27\% effective), the samples had $4.3 \%$ air voids and $14.5 \%$ VMA. 
Table 2.2.1: HMA design parameters for mixes 1 to 4 (Timm and Priest, 2006).

\begin{tabular}{|l|c|c|c|c|c|}
\hline \multicolumn{1}{|c|}{ Property } & Units & Mix 1 & Mix 2 & Mix 3 & Mix 4 \\
\hline Binder Grade & - & \multicolumn{5}{c|}{$76-22$} & \multicolumn{2}{c|}{80} \\
\hline Compactive Effort, & gyrations & \multicolumn{5}{c|}{} \\
\hline Mixing Temperature & ${ }^{\circ} \mathrm{F}\left({ }^{\circ} \mathrm{C}\right)$ & \multicolumn{2}{|c|}{$345(174)$} & $325(163)$ \\
\hline Effective Binder Content & percent & 6.13 & 4.27 & 6.13 & 4.27 \\
\hline Dust to Binder Ratio & - & 0.88 & 1.10 & 0.88 & 1.10 \\
\hline \multirow{2}{*}{ Bulk Unit Weight of Compacted Pills } & $\begin{array}{c}\mathrm{pcf} \\
\left(\mathrm{kg} / \mathrm{m}^{3}\right)\end{array}$ & $\begin{array}{c}147.8 \\
(2370)\end{array}$ & $\begin{array}{c}153.6 \\
(2463)\end{array}$ & $\begin{array}{c}147.8 \\
(2370)\end{array}$ & $\begin{array}{c}\text { (2463) } \\
\text { (24) }\end{array}$ \\
\hline Air Void Content & percent & \multicolumn{5}{|c|}{4.3} \\
\hline Voids in Mineral Aggregate & percent & 17.9 & 14.5 & 17.9 & 14.5 \\
\hline
\end{tabular}

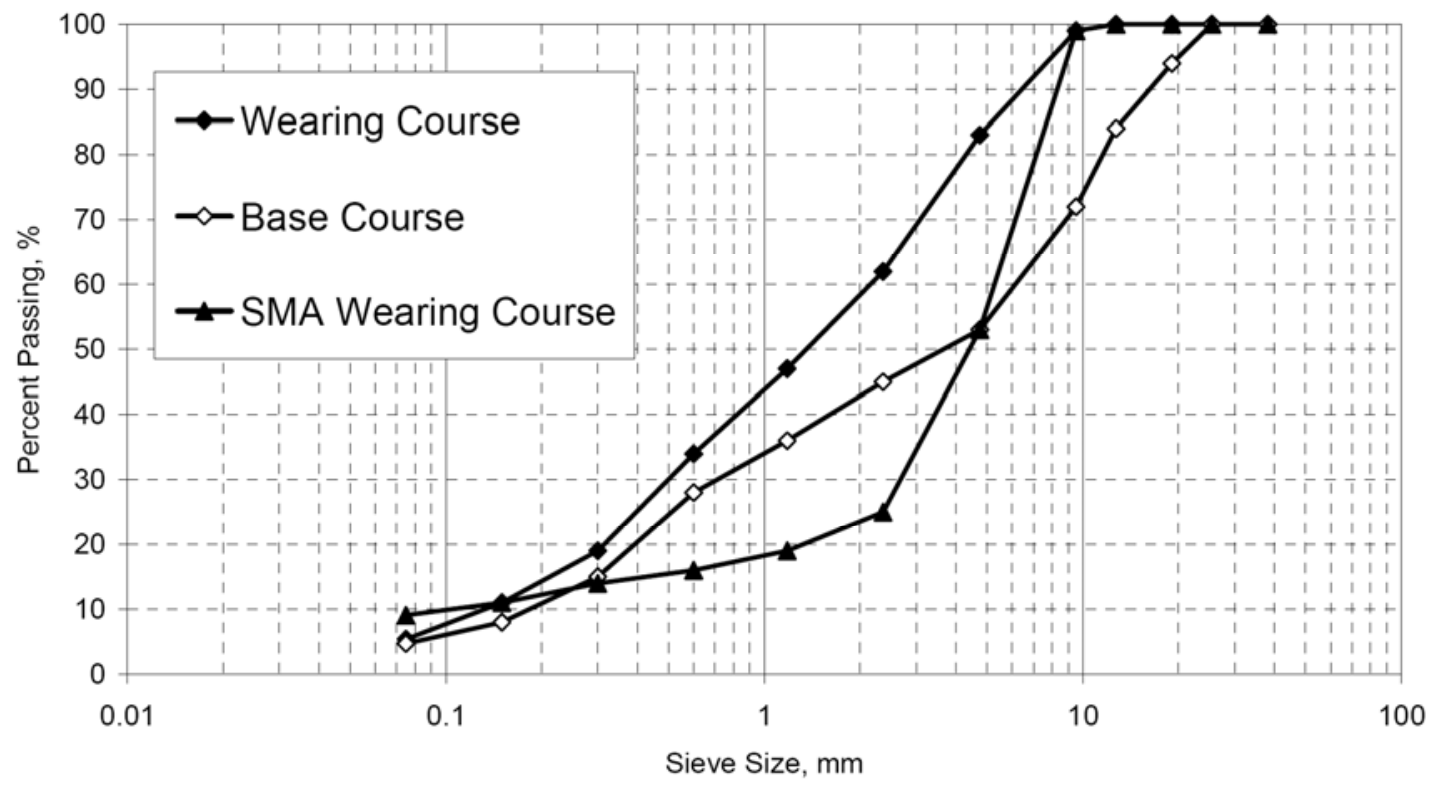

Figure 2.2.5: Design gradation of different mix types used in the NCAT 'structural study’ (Timm and Priest, 2006).

Surveys of the as-built thicknesses were conducted during construction. These focused on the pavement areas that included instrumentation. The average thicknesses of individual lifts were as follows. For section N1, the bottom lift was 2.2 in. (56 mm) thick, the intermediate was $2.1 \mathrm{in.} \mathrm{(53} \mathrm{mm)} \mathrm{thick,} \mathrm{and} \mathrm{the} \mathrm{top} \mathrm{lift} \mathrm{was} 0.6 \mathrm{in}$. (15 mm) thick. For section N2, the bottom lift was $1.8 \mathrm{in}$. (46 mm) thick, the intermediate lift was $2.0 \mathrm{in.}(51 \mathrm{~mm})$, and the top lift was $1.1 \mathrm{in}$. $(28 \mathrm{~mm})$ thick. Therefore, the average total HMA thickness over the instrumented areas was 4.9 in. (124 mm) in both sections. Cores taken after construction confirmed these results. It should be noted that the HMA 
thickness in Section N1 was not constant at 5.0 in. $(127 \mathrm{~mm})$, but varied between 6.7 to 4.5 in. (170 to $114 \mathrm{~mm})$.

The as-built air void contents were also surveyed during construction. On average, all three lifts in Section N1 were compacted to an air void content of $7.0 \%$. However, in Section N2 only the top lift was compacted to $7.0 \%$ voids while the two bottom lifts were compacted to an air void content of $6.0 \%$. The asphalt content also varied slightly relative to the design values. Additional details regarding the HMA construction can be found in Powell and Brown (2004).

\subsection{MECHANICAL TESTING}

\subsubsection{Resilient Modulus of Subgrade and Base Materials}

The resilient modulus of the upper subgrade soil was tested according to the AASHTO T307 protocol. Tests were done either on recompacted material or on undisturbed specimens obtained from the field after subgrade construction. The test conditions included three levels of moisture content (denoted by $\omega$ ): $7.2 \%, 9.7 \%$ and $20.1 \%$; three levels of applied confining pressure: 2, 4 and 6 psi (13.8, 27.6 and $41.4 \mathrm{kPa}$ ); and five levels of applied cyclic axial stress: 2, 4, 6, 8 and 10 psi (13.8, 27.6, 41.4 and $68.9 \mathrm{kPa})$. The average dry density of the tested samples was $114.4 \mathrm{pcf}\left(1834 \mathrm{~kg} / \mathrm{m}^{3}\right)$ which

represents a relative compaction degree of about $96 \%$ (recall that the compaction degree in the field was about $100 \%)$. The raw test results are presented in Table 2.3.1. These were obtained directly from the laboratory reports.

Timm and Priest (2006) provided three regression equations, each fitting the test results for a single level of moisture content; the mathematical expression they used was as follows:

$$
M_{R}=b_{1} \cdot\left(S_{c}\right)^{b_{2}}\left(S_{3}\right)^{b_{3}}
$$

where $M_{R}$ is the resilient modulus (in psi), $b_{1}, b_{2}$ and $b_{3}$ are regression constants, $S_{c}$ is the peak applied uniaxial cyclic stress (in psi), and $S_{3}$ is the applied confining pressure (in psi). 
Table 2.3.1: Resilient modulus of subgrade soil with average compaction level of 96\%. Raw test results from laboratory reports.

\begin{tabular}{|c|c|c|c|c|c|c|}
\hline \multirow[t]{2}{*}{$\begin{array}{l}\text { Confining } \\
\text { Pressure, } \\
\text { psi (kPa) }\end{array}$} & $\begin{array}{c}\text { Peak } \\
\text { Cyclic } \\
\text { Stress } \\
\text { psi } \\
\text { (kPa) }\end{array}$ & $\begin{array}{l}\text { Resilient } \\
\text { Modulus, } \\
\text { psi (MPa) }\end{array}$ & $\begin{array}{c}\text { Peak } \\
\text { Cyclic } \\
\text { Stress, } \\
\text { psi } \\
\text { (kPa) }\end{array}$ & $\begin{array}{l}\text { Resilient } \\
\text { Modulus, } \\
\text { psi (MPa) }\end{array}$ & $\begin{array}{c}\text { Peak } \\
\text { Cyclic } \\
\text { Stress, } \\
\text { psi } \\
\text { (kPa) }\end{array}$ & $\begin{array}{l}\text { Resilient } \\
\text { Modulus, } \\
\text { psi (MPa) }\end{array}$ \\
\hline & \multicolumn{2}{|c|}{$\omega=7.2 \%$} & \multicolumn{2}{|c|}{$\omega=9.7 \%$} & \multicolumn{2}{|c|}{$\omega=20.1 \%$} \\
\hline \multirow{5}{*}{$\begin{array}{c}6.0 \\
(41.4)\end{array}$} & $\begin{array}{c}2.1 \\
(14.5)\end{array}$ & $\begin{array}{c}10,876 \\
(75.0)\end{array}$ & $\begin{array}{c}2.1 \\
(14.5)\end{array}$ & $\begin{array}{l}9,970 \\
(68.7)\end{array}$ & $\begin{array}{c}2.2 \\
(15.2)\end{array}$ & $\begin{array}{c}10,034 \\
(69.2)\end{array}$ \\
\hline & $\begin{array}{c}4.0 \\
(27.6)\end{array}$ & $\begin{array}{c}11,738 \\
(80.9) \\
\end{array}$ & $\begin{array}{c}4.0 \\
(27.6)\end{array}$ & $\begin{array}{l}11,177 \\
(77.1) \\
\end{array}$ & $\begin{array}{c}4.1 \\
(28.3)\end{array}$ & $\begin{array}{c}10,653 \\
(73.4)\end{array}$ \\
\hline & $\begin{array}{c}5.8 \\
(40.0)\end{array}$ & $\begin{array}{c}11,730 \\
(80.9)\end{array}$ & $\begin{array}{c}5.9 \\
(40.7)\end{array}$ & $\begin{array}{c}11,478 \\
(79.1)\end{array}$ & $\begin{array}{c}5.9 \\
(40.7)\end{array}$ & $\begin{array}{c}10,568 \\
(72.9)\end{array}$ \\
\hline & $\begin{array}{c}7.7 \\
(53.1)\end{array}$ & $\begin{array}{c}12,260 \\
(84.5) \\
\end{array}$ & $\begin{array}{c}7.7 \\
(53.1)\end{array}$ & $\begin{array}{c}11,922 \\
(82.2) \\
\end{array}$ & $\begin{array}{c}7.7 \\
(53.1)\end{array}$ & $\begin{array}{c}10,511 \\
(72.5)\end{array}$ \\
\hline & $\begin{array}{c}9.6 \\
(66.2)\end{array}$ & $\begin{array}{l}12,837 \\
(88.5)\end{array}$ & $\begin{array}{c}9.6 \\
(66.2)\end{array}$ & $\begin{array}{c}12,384 \\
(85.4)\end{array}$ & $\begin{array}{c}9.6 \\
(66.2)\end{array}$ & $\begin{array}{c}10,477 \\
(72.2)\end{array}$ \\
\hline \multirow{5}{*}{$\begin{array}{c}4.0 \\
(27.6)\end{array}$} & $\begin{array}{c}2.1 \\
(14.5) \\
\end{array}$ & $\begin{array}{l}10,311 \\
(71.1) \\
\end{array}$ & $\begin{array}{c}2.0 \\
(13.8) \\
\end{array}$ & $\begin{array}{l}7,310 \\
(50.4) \\
\end{array}$ & $\begin{array}{c}2.1 \\
(14.5) \\
\end{array}$ & $\begin{array}{l}8,502 \\
(58.6) \\
\end{array}$ \\
\hline & $\begin{array}{c}3.9 \\
(26.9) \\
\end{array}$ & $\begin{array}{l}10,222 \\
(70.5) \\
\end{array}$ & $\begin{array}{c}3.9 \\
(26.9) \\
\end{array}$ & $\begin{array}{l}7,762 \\
(53.5) \\
\end{array}$ & $\begin{array}{c}4.0 \\
(27.6) \\
\end{array}$ & $\begin{array}{l}8,614 \\
(59.4) \\
\end{array}$ \\
\hline & $\begin{array}{c}5.7 \\
(39.3) \\
\end{array}$ & $\begin{array}{l}10,252 \\
(70.7) \\
\end{array}$ & $\begin{array}{c}5.9 \\
(40.7) \\
\end{array}$ & $\begin{array}{l}8,487 \\
(58.5) \\
\end{array}$ & $\begin{array}{c}5.9 \\
(40.7) \\
\end{array}$ & $\begin{array}{l}8,736 \\
(60.2) \\
\end{array}$ \\
\hline & $\begin{array}{c}7.6 \\
(52.4) \\
\end{array}$ & $\begin{array}{l}10,465 \\
(72.2) \\
\end{array}$ & $\begin{array}{c}7.8 \\
(53.8) \\
\end{array}$ & $\begin{array}{l}9,225 \\
(63.6) \\
\end{array}$ & $\begin{array}{c}7.7 \\
(53.1) \\
\end{array}$ & $\begin{array}{l}8,854 \\
(61.0) \\
\end{array}$ \\
\hline & $\begin{array}{c}9.5 \\
(65.5) \\
\end{array}$ & $\begin{array}{l}10,965 \\
(75.6) \\
\end{array}$ & $\begin{array}{c}9.7 \\
(66.9) \\
\end{array}$ & $\begin{array}{l}9,987 \\
(68.9) \\
\end{array}$ & $\begin{array}{c}9.6 \\
(66.2) \\
\end{array}$ & $\begin{array}{l}9,101 \\
(62.7) \\
\end{array}$ \\
\hline \multirow{5}{*}{$\begin{array}{c}2.0 \\
(13.8)\end{array}$} & $\begin{array}{c}1.9 \\
(13.1) \\
\end{array}$ & $\begin{array}{l}7,871 \\
(54.3) \\
\end{array}$ & $\begin{array}{c}2.0 \\
(13.8) \\
\end{array}$ & $\begin{array}{l}4,339 \\
(29.9) \\
\end{array}$ & $\begin{array}{c}2.0 \\
(13.8) \\
\end{array}$ & $\begin{array}{l}5,032 \\
(34.7) \\
\end{array}$ \\
\hline & $\begin{array}{c}3.8 \\
(26.2) \\
\end{array}$ & $\begin{array}{l}7,783 \\
(53.7) \\
\end{array}$ & $\begin{array}{c}4.0 \\
(27.6)\end{array}$ & $\begin{array}{l}4,701 \\
(32.4) \\
\end{array}$ & $\begin{array}{c}4.0 \\
(27.6) \\
\end{array}$ & $\begin{array}{l}5,451 \\
(37.6) \\
\end{array}$ \\
\hline & $\begin{array}{c}5.6 \\
(38.6) \\
\end{array}$ & $\begin{array}{l}7,920 \\
(54.6) \\
\end{array}$ & $\begin{array}{c}6.0 \\
(41.4) \\
\end{array}$ & $\begin{array}{l}5,507 \\
(38.0) \\
\end{array}$ & $\begin{array}{c}5.9 \\
(40.7) \\
\end{array}$ & $\begin{array}{l}6,119 \\
(42.2) \\
\end{array}$ \\
\hline & $\begin{array}{c}7.5 \\
(51.7) \\
\end{array}$ & $\begin{array}{l}8,216 \\
(56.6) \\
\end{array}$ & $\begin{array}{c}7.9 \\
(54.5) \\
\end{array}$ & $\begin{array}{l}6,285 \\
(43.3) \\
\end{array}$ & $\begin{array}{c}7.8 \\
(53.8) \\
\end{array}$ & $\begin{array}{l}6,641 \\
(45.8) \\
\end{array}$ \\
\hline & $\begin{array}{c}9.5 \\
(65.5) \\
\end{array}$ & $\begin{array}{l}8,776 \\
(60.5) \\
\end{array}$ & $\begin{array}{c}9.7 \\
(66.9) \\
\end{array}$ & $\begin{array}{l}7,084 \\
(48.8) \\
\end{array}$ & $\begin{array}{c}9.7 \\
(66.9) \\
\end{array}$ & $\begin{array}{l}7,149 \\
(49.3) \\
\end{array}$ \\
\hline
\end{tabular}

The resilient modulus of the base material was also tested according to AASHTO T307 procedure. Tests were done on specimens recompacted to a dry density of $128.6 \mathrm{pcf}\left(2062 \mathrm{~kg} / \mathrm{m}^{3}\right)$. This value represents a relatively low compaction degree of 
about $93 \%$ (recall that $100 \%$ compaction was achieved in the field). The test conditions included two levels of moisture content ( $\omega$ ): 5.3\% and 9.8\%; three levels of applied confining pressure: 2, 4 and 6 psi (13.8, 27.6 and $41.4 \mathrm{kPa})$; and five levels of applied cyclic axial stress between 2 and 10 psi (13.8 to $68.9 \mathrm{kPa}$ ). The raw test results are given in Table 2.3.2.

Table 2.3.2: Resilient modulus of aggregate base with average compaction level of $93 \%$. Raw test results from laboratory reports.

\begin{tabular}{|c|c|c|c|c|}
\hline $\begin{array}{c}\text { Confining } \\
\text { Pressure, psi } \\
\text { (kPa) }\end{array}$ & $\begin{array}{c}\text { Peak Cyclic } \\
\text { Stress, psi } \\
\text { (kPa) }\end{array}$ & $\begin{array}{c}\text { Resilient } \\
\text { Modulus, psi } \\
\text { (MPa) }\end{array}$ & $\begin{array}{c}\text { Peak Cyclic } \\
\text { Stress, psi } \\
\text { (kPa) }\end{array}$ & $\begin{array}{c}\text { Resilient } \\
\text { Modulus, psi } \\
\text { (MPa) }\end{array}$ \\
\cline { 2 - 5 } & \multicolumn{2}{|c|}{$\boldsymbol{\omega}=\mathbf{5 . 3 \%}$} & \multicolumn{2}{c|}{$\boldsymbol{\omega}=\mathbf{9 . 8 \%}$} \\
\hline \multirow{4}{*}{6.0} & $2.2(15.2)$ & $9,062(62.5)$ & $2.1(14.5)$ & $8,698(60.0)$ \\
\cline { 2 - 5 }$(41.4)$ & $4.1(28.3)$ & $9,236(63.7)$ & $4.0(27.6)$ & $8,950(61.7)$ \\
\cline { 2 - 5 } & $5.9(40.7)$ & $9,080(62.6)$ & $5.8(40.0)$ & $8,785(60.6)$ \\
\cline { 2 - 5 } & $7.8(53.8)$ & $9,017(62.2)$ & $7.6(52.4)$ & $8,751(60.3)$ \\
\cline { 2 - 5 } & $9.7(66.9)$ & $9,138(63.0)$ & $9.5(65.5)$ & $8,864(61.1)$ \\
\hline \multirow{4}{*}{4.0} & $2.1(14.5)$ & $7,615(52.5)$ & $2.1(14.5)$ & $6,830(47.1)$ \\
\cline { 2 - 5 }$(27.6)$ & $3.9(26.9)$ & $7,091(48.9)$ & $4.0(27.6)$ & $6,322(43.6)$ \\
\cline { 2 - 5 } & $5.8(40.0)$ & $6,986(48.2)$ & $5.9(40.7)$ & $6,366(43.9)$ \\
\cline { 2 - 5 } & $7.7(53.1)$ & $7,164(49.4)$ & $7.7(53.1)$ & $6,642(45.8)$ \\
\cline { 2 - 5 } & $9.7(66.9)$ & $7,481(51.6)$ & $9.5(65.5)$ & $6,945(47.9)$ \\
\hline \multirow{4}{*}{2.0} & $2.0(13.8)$ & $5,194(35.8)$ & $2.0(13.8)$ & $4,280(29.5)$ \\
\cline { 2 - 5 }$(13.8)$ & $3.8(26.2)$ & $4,843(33.4)$ & $3.9(26.9)$ & $3,966(27.3)$ \\
\cline { 2 - 5 } & $5.7(39.3)$ & $4,983(34.4)$ & $5.9(40.7)$ & $4,233(29.2)$ \\
\cline { 2 - 5 } & $7.7(53.1)$ & $5,339(36.8)$ & $7.7(53.1)$ & $4,643(32.0)$ \\
\cline { 2 - 5 } & $9.7(66.9)$ & $5,731(39.5)$ & $9.5(65.5)$ & $5,003(34.5)$ \\
\hline
\end{tabular}

Another set of tests was done on the base material with a relative compaction degree of $97.5 \%$ and a moisture content of $5.5 \%$. These tests were done under applied confining pressure levels ranging from 3 to $20 \mathrm{psi}$ (20.7 to $137.9 \mathrm{kPa}$ ), and cyclic stress levels in the range of 3 to $40 \mathrm{psi}$ (20.7 to $275.8 \mathrm{kPa}$ ). Relating to equation 2.1, the obtained coefficients were: $b_{1}=5677, b_{2}=0$ and $b_{3}=0.4711$. 


\subsubsection{HMA Complex Modulus}

Complex modulus testing of the NCAT asphalt mixtures was conducted under the direction of Dr. Terhi Pellinen at Purdue University. A detailed description of the work is presented in a report by Barde and Cardone (2004) which can be found in Appendix C. The materials were sampled in loose state from the delivery trucks during construction and then shipped to Purdue University. Specimens were prepared and tested in accordance with the NCHRP 1-37A protocol (NCHRP, 2002).

Initially, mixtures were heated to compaction temperature in the oven; a temperature of $155^{\circ} \mathrm{C}$ was selected for mixes 1 and 3 , and a temperature of $145^{\circ} \mathrm{C}$ was selected for mixes 2 and 4 . Next, specimens were prepared using a gyratory compactor. For each mixture type, four specimens were prepared for testing (i.e., a total of 16) by targeting an air void content of 7\%. The first two specimens, denoted by 'a' and 'b' in the report, were compacted with assumed correction factors (i.e., ratio between sample bulk specific gravity that is measured using saturated surface-day method and bulk specific gravity that is calculated based on sample height in the gyratory mold). The next two specimens, denoted by ' $c$ ' and ' $d$ ' in the report, were prepared with calibrated correction factors after studying the resulting densities of the first two.

The gyratory compacted pills were further cored to produce cylindrical specimens 4 in. $(102 \mathrm{~mm})$ in diameter and 6 in. $(152 \mathrm{~mm})$ in height. The final air void content for the 16 samples varied in the range of 6.8 to $8.2 \%$. Complex modulus tests were done on each of the 16 samples in stress-controlled uniaxial compression mode with a Haversine load pattern. Six different frequencies were employed: 25, 10, 5, 1, 0.5 and $0.1 \mathrm{~Hz}$; applied in descending order. The frequency sweep was executed five times for five different temperature levels: $-10,4.4,21.1,37.8$ and $54.4{ }^{\circ} \mathrm{C}$; applied in ascending order.

A dummy specimen was used to verify that the target temperature level was reached. The testing was carried out using a UTM 25 with a $25 \mathrm{kN}$ load cell. Stress levels were adjusted in order to maintain the values of both permanent and recoverable strain within specified protocol limits. In an effort to minimize edge effects, latex sheets covered with silicon grease were inserted between loading system and specimen at both 
ends. Specimens were instrumented with three linear variable displacement transformers (LVDTs), each spanning $100 \mathrm{~mm}$, for measurement of axial (vertical) deformations; the LVDTs were mounted to the periphery of the specimen $120^{\circ}$ apart. It should be noted that lateral strains were not monitored.

The complex modulus test results are shown in Tables 2.3.3 to 2.3.6. Each table presents the dynamic moduli values and the corresponding phase angles. It was found, separately for each mix, that the initial differences in voids did not affect the test results in a statistically significant manner. Therefore, each value in these tables constitutes the average result of four separate tests. 
Table 2.3.3: Average complex modulus test results for Mix 1 (Barde and Cardone, 2004).

\begin{tabular}{|c|c|c|c|}
\hline $\begin{array}{c}\text { Test Temperature } \\
{ }^{\circ} \mathrm{C}\left({ }^{\circ} \mathrm{F}\right)\end{array}$ & $\begin{array}{c}\text { Test Frequency } \\
{\left[\begin{array}{l}{[\mathrm{Hz}]}\end{array}\right.}\end{array}$ & $\begin{array}{c}\text { Dynamic Modulus } \\
\text { MPa (ksi) }\end{array}$ & $\begin{array}{c}\text { Phase Angle } \\
\text { [degrees] }\end{array}$ \\
\hline \multirow{6}{*}{$\begin{array}{l}-10.0 \\
(+14)\end{array}$} & 25 & $23,057(3,344)$ & 6.1 \\
\hline & 10 & $21,808(3,163)$ & 9.2 \\
\hline & 5 & $20,585(2,986)$ & 9.7 \\
\hline & 1 & $18,129(2,629)$ & 10.9 \\
\hline & 0.5 & $17,017(2,468)$ & 11.5 \\
\hline & 0.1 & $14,364(2,083)$ & 13.2 \\
\hline \multirow{6}{*}{$\begin{array}{c}+4.4 \\
(+40)\end{array}$} & 25 & $13,792(2,000)$ & 10.1 \\
\hline & 10 & $12,365(1,793)$ & 11.2 \\
\hline & 5 & $11,418(1,656)$ & 13.6 \\
\hline & 1 & $9,391(1,362)$ & 15.3 \\
\hline & 0.5 & $8,563(1,242)$ & 16.3 \\
\hline & 0.1 & $6,722(975)$ & 18.9 \\
\hline \multirow{6}{*}{$\begin{array}{l}+21.1 \\
(+70)\end{array}$} & 25 & $7,099(1,030)$ & 16.0 \\
\hline & 10 & $5,983(868)$ & 17.0 \\
\hline & 5 & $5,052(733)$ & 23.1 \\
\hline & 1 & $3,529(512)$ & 26.5 \\
\hline & 0.5 & $3,009(436)$ & 27.4 \\
\hline & 0.1 & 2,061 (299) & 29.3 \\
\hline \multirow{6}{*}{$\begin{array}{c}+37.8 \\
(+100)\end{array}$} & 25 & $2,622(380)$ & 27.5 \\
\hline & 10 & $2,139(310)$ & 23.5 \\
\hline & 5 & $1,694(246)$ & 25.3 \\
\hline & 1 & $1,020(148)$ & 33.6 \\
\hline & 0.5 & 862 (125) & 32.3 \\
\hline & 0.1 & $589(85)$ & 30.2 \\
\hline \multirow{6}{*}{$\begin{array}{l}+54.4 \\
(+130)\end{array}$} & 25 & $1,054(153)$ & 22.2 \\
\hline & 10 & $855(124)$ & 15.1 \\
\hline & 5 & 685 (99) & 16.6 \\
\hline & 1 & $436(63)$ & 21.1 \\
\hline & 0.5 & $382(55)$ & 22.8 \\
\hline & 0.1 & $286(41)$ & 26.9 \\
\hline
\end{tabular}


Table 2.3.4: Average complex modulus test results for Mix 2 (Barde and Cardone, 2004).

\begin{tabular}{|c|c|c|c|}
\hline $\begin{array}{l}\text { Test Temperature } \\
{ }^{\circ} \mathrm{C}\left({ }^{\circ} \mathrm{F}\right)\end{array}$ & $\begin{array}{c}\text { Test Frequency } \\
{[\mathrm{Hz}]}\end{array}$ & $\begin{array}{c}\text { Dynamic Modulus } \\
\text { MPa (ksi) }\end{array}$ & $\begin{array}{c}\text { Phase Angle } \\
\text { [degrees] }\end{array}$ \\
\hline \multirow{6}{*}{$\begin{array}{l}-10.0 \\
(+14)\end{array}$} & 25 & $25,440(3,690)$ & 6.6 \\
\hline & 10 & $24,150(3,503)$ & 9.7 \\
\hline & 5 & $22,781(3,304)$ & 10.5 \\
\hline & 1 & $20,048(2,908)$ & 12.1 \\
\hline & 0.5 & $18,791(2,725)$ & 12.8 \\
\hline & 0.1 & $15,703(2,278)$ & 15.1 \\
\hline \multirow{6}{*}{$\begin{array}{l}+4.4 \\
(+40)\end{array}$} & 25 & $16,581(2,405)$ & 10.9 \\
\hline & 10 & $14,813(2,148)$ & 12.2 \\
\hline & 5 & $13,710(1,988)$ & 13.9 \\
\hline & 1 & $11,191(1,623)$ & 16.0 \\
\hline & 0.5 & $10,168(1,475)$ & 17.2 \\
\hline & 0.1 & $7,951(1,153)$ & 20.4 \\
\hline \multirow{6}{*}{$\begin{array}{l}+21.1 \\
(+70)\end{array}$} & 25 & $8,575(1,244)$ & 15.3 \\
\hline & 10 & $7,321(1,062)$ & 15.3 \\
\hline & 5 & $6,302(914)$ & 22.6 \\
\hline & 1 & 4,503 (653) & 25.0 \\
\hline & 0.5 & $3,898(565)$ & 26.1 \\
\hline & 0.1 & 2,722 (395) & 28.6 \\
\hline \multirow{6}{*}{$\begin{array}{c}+37.8 \\
(+100)\end{array}$} & 25 & $4,238(615)$ & 26.2 \\
\hline & 10 & $3,498(507)$ & 27.3 \\
\hline & 5 & 2,792 (405) & 24.9 \\
\hline & 1 & $1,752(254)$ & 32.5 \\
\hline & 0.5 & $1,481(215)$ & 32.1 \\
\hline & 0.1 & $1,044(151)$ & 31.2 \\
\hline \multirow{6}{*}{$\begin{array}{c}+54.4 \\
(+130)\end{array}$} & 25 & $1,504(218)$ & 26.2 \\
\hline & 10 & $1,200(174)$ & 22.7 \\
\hline & 5 & 985 (143) & 21.5 \\
\hline & 1 & 655 (95) & 25.4 \\
\hline & 0.5 & $578(84)$ & 26.4 \\
\hline & 0.1 & $450(65)$ & 27.4 \\
\hline
\end{tabular}


Table 2.3.5: Average complex modulus test results for Mix 3 (Barde and Cardone, 2004).

\begin{tabular}{|c|c|c|c|}
\hline $\begin{array}{l}\text { Test Temperature } \\
{ }^{\circ} \mathbf{C}\left({ }^{\circ} \mathbf{F}\right)\end{array}$ & $\begin{array}{c}\text { Test Frequency } \\
{[\mathrm{Hz}]}\end{array}$ & $\begin{array}{c}\text { Dynamic Modulus } \\
\text { MPa (ksi) }\end{array}$ & $\begin{array}{c}\text { Phase Angle } \\
\text { [degrees] }\end{array}$ \\
\hline \multirow{6}{*}{$\begin{array}{l}-10.0 \\
(+14)\end{array}$} & 25 & $22,559(3,272)$ & 4.9 \\
\hline & 10 & $21,386(3,102)$ & 7.8 \\
\hline & 5 & $20,300(2,944)$ & 8.9 \\
\hline & 1 & $17,801(2,582)$ & 10.5 \\
\hline & 0.5 & $16,667(2,417)$ & 11.3 \\
\hline & 0.1 & $13,991(2,029)$ & 13.5 \\
\hline \multirow{6}{*}{$\begin{array}{c}+4.4 \\
(+40)\end{array}$} & 25 & $11,884(1,724)$ & 11.2 \\
\hline & 10 & $10,786(1,564)$ & 13.1 \\
\hline & 5 & $9,879(1,433)$ & 15.6 \\
\hline & 1 & $7,858(1,140)$ & 17.6 \\
\hline & 0.5 & $7,111(1,031)$ & 18.9 \\
\hline & 0.1 & 5,457 (791) & 21.8 \\
\hline \multirow{6}{*}{$\begin{array}{l}+21.1 \\
(+70)\end{array}$} & 25 & $7,113(1,032)$ & 16.7 \\
\hline & 10 & $5,928(860)$ & 19.1 \\
\hline & 5 & $5,087(738)$ & 21.9 \\
\hline & 1 & 3,504 (508) & 27.3 \\
\hline & 0.5 & 2,970 (431) & 28.0 \\
\hline & 0.1 & $1,992(289)$ & 30.1 \\
\hline \multirow{6}{*}{$\begin{array}{c}+37.8 \\
(+100)\end{array}$} & 25 & $2,634(382)$ & 24.3 \\
\hline & 10 & 2,038 (296) & 20.5 \\
\hline & 5 & $1,624(236)$ & 23.7 \\
\hline & 1 & $1,008(146)$ & 28.7 \\
\hline & 0.5 & 851 (123) & 28.6 \\
\hline & 0.1 & 604 (88) & 27.9 \\
\hline \multirow{6}{*}{$\begin{array}{l}+54.4 \\
(+130)\end{array}$} & 25 & $673(98)$ & 31.2 \\
\hline & 10 & $534(77)$ & 28.9 \\
\hline & 5 & $448(65)$ & 29.7 \\
\hline & 1 & $313(45)$ & 26.5 \\
\hline & 0.5 & $276(40)$ & 25.4 \\
\hline & 0.1 & $216(31)$ & 24.1 \\
\hline
\end{tabular}


Table 2.3.6: Average complex modulus test results for Mix 4 (Barde and Cardone, 2004).

\begin{tabular}{|c|c|c|c|}
\hline $\begin{array}{c}\text { Test Temperature } \\
{ }^{\circ} \mathbf{C}\left({ }^{\circ} \mathbf{F}\right)\end{array}$ & $\begin{array}{c}\text { Test Frequency } \\
{[\mathrm{Hz}]}\end{array}$ & $\begin{array}{c}\text { Dynamic Modulus } \\
\text { MPa (ksi) }\end{array}$ & $\begin{array}{c}\text { Phase Angle } \\
\text { [degrees] }\end{array}$ \\
\hline \multirow{6}{*}{$\begin{array}{l}-10.0 \\
(+14)\end{array}$} & 25 & $24,940(3,617)$ & 5.8 \\
\hline & 10 & $23,592(3,422)$ & 7.9 \\
\hline & 5 & $22,678(3,289)$ & 9.1 \\
\hline & 1 & $19,975(2,897)$ & 10.6 \\
\hline & 0.5 & $18,783(2,724)$ & 11.5 \\
\hline & 0.1 & $15,847(2,298)$ & 13.6 \\
\hline \multirow{6}{*}{$\begin{array}{c}+4.4 \\
(+40)\end{array}$} & 25 & $16,045(2,327)$ & 10.5 \\
\hline & 10 & $14,393(2,088)$ & 13.0 \\
\hline & 5 & $13,241(1,920)$ & 14.8 \\
\hline & 1 & $10,641(1,543)$ & 17.4 \\
\hline & 0.5 & $9,622(1,396)$ & 18.7 \\
\hline & 0.1 & $7,394(1,072)$ & 22.3 \\
\hline \multirow{6}{*}{$\begin{array}{l}+21.1 \\
(+70)\end{array}$} & 25 & $9,036(1,311)$ & 16.9 \\
\hline & 10 & $7,341(1,065)$ & 22.0 \\
\hline & 5 & $6,419(931)$ & 23.0 \\
\hline & 1 & $4,436(643)$ & 26.7 \\
\hline & 0.5 & $3,772(547)$ & 28.2 \\
\hline & 0.1 & 2,612 (379) & 30.9 \\
\hline \multirow{6}{*}{$\begin{array}{c}+37.8 \\
(+100)\end{array}$} & 25 & $3,958(574)$ & 23.4 \\
\hline & 10 & $3,072(446)$ & 23.9 \\
\hline & 5 & $2,480(360)$ & 28.1 \\
\hline & 1 & $1,608(233)$ & 30.1 \\
\hline & 0.5 & $1,363(198)$ & 29.8 \\
\hline & 0.1 & $962(140)$ & 29.4 \\
\hline \multirow{6}{*}{$\begin{array}{c}+54.4 \\
(+130)\end{array}$} & 25 & $1,485(215)$ & 24.0 \\
\hline & 10 & 1,157 (168) & 16.5 \\
\hline & 5 & 931 (135) & 17.2 \\
\hline & 1 & $619(90)$ & 20.7 \\
\hline & 0.5 & $548(79)$ & 21.6 \\
\hline & 0.1 & 435 (63) & 24.6 \\
\hline
\end{tabular}




\subsubsection{Falling Weight Deflections}

Falling weight deflectometer (FWD) testing was conducted more or less on a monthly basis at NCAT. These tests were conducted at identical locations within each test section. The locations themselves were randomly selected at the beginning of the entire two year experiment. The FWD was a Dynatest 8000 model equipped with seven sensors spaced at 12 in. (304.8 mm) intervals starting from the center of the load plate. The load plate had a radius of $300 \mathrm{~mm}$ (5.91 in.) and was equipped with a split configuration to ensure better contact with the pavement surface. For each location, two drops of about 9,000 lb (4,086 kg) load were executed.

Between November 3, 2003, and February 7, 2005, 135 FWD tests were conducted on sections N1 and N2 (i.e., 270 drops). The testing locations were later cored to obtain the as-build pavement thicknesses. The data provided by Table 2.3.7 consists only of those deflections (average of two drops) conducted over a point located inside the wheel path for which the HMA thickness was verified via coring to be exactly 5.0 in. $(127 \mathrm{~mm})$. It should be noted that the first few deflection bowls characterize the N1 pavement in its pristine state, soon after construction and before it had incurred significant damage. Similarly, Table 2.3.8 includes the results for section N2. Both tables show the HMA temperature at the time of testing measured at a depth of $50.8 \mathrm{~mm}$ ( 2 in.) from the surface. Also, for preparing both tables the measured deflections were linearly normalized to a peak load of exactly 9,000 lb (4,086 kg) in magnitude. 
Table 2.3.7: FWD deflections at N1 section (location according to NCAT database: station 2 inside the wheel path).

\begin{tabular}{|l|c|c|c|c|c|c|c|c|}
\hline \multicolumn{1}{|c|}{ Date } & $\begin{array}{c}\text { D0 } \\
{[\boldsymbol{\mu m}]}\end{array}$ & $\begin{array}{c}\mathbf{D 1} \\
{[\boldsymbol{\mu \mathbf { m } ]}}\end{array}$ & $\begin{array}{c}\mathbf{D 2} \\
{[\boldsymbol{\mu m}]}\end{array}$ & $\begin{array}{c}\mathbf{D 3} \\
{[\boldsymbol{\mu m}]}\end{array}$ & $\begin{array}{c}\mathbf{D 4} \\
{[\boldsymbol{\mu m}]}\end{array}$ & $\begin{array}{c}\mathbf{D 5} \\
{[\boldsymbol{\mu m}]}\end{array}$ & $\begin{array}{c}\text { D6 } \\
{[\boldsymbol{\mu m}]}\end{array}$ & $\begin{array}{c}\text { Temp. } \\
{ }^{\mathbf{o}} \mathbf{C}\left({ }^{\mathbf{o}} \mathbf{F}\right)\end{array}$ \\
\hline Nov. 3, 2003 & 565.7 & 353.0 & 153.4 & 76.9 & 40.9 & 32.1 & 20.7 & $32.9(91.2)$ \\
\hline Dec. 15, 2003 & 451.2 & 332.5 & 178.4 & 93.9 & 47.6 & 31.2 & 24.2 & $16.7(62.1)$ \\
\hline Jan. 26, 2004 & 338.1 & 263.2 & 160.4 & 99.2 & 60.9 & 38.0 & 29.5 & $10.1(50.2)$ \\
\hline Feb. 23, 2004 & 354.0 & 268.1 & 160.3 & 92.1 & 52.7 & 30.9 & 22.5 & $12.6(54.7)$ \\
\hline Mar. 22, 2004 & 620.2 & 416.7 & 200.7 & 99.6 & 55.3 & 36.0 & 26.3 & $26.8(80.2)$ \\
\hline June 14, 2004 & 771.7 & 511.9 & 224.4 & 101.6 & 53.3 & 34.6 & 26.7 & $32.3(90.1)$ \\
\hline Sep. 20, 2004 & 841.3 & 545.0 & 237.4 & 94.8 & 44.1 & 36.6 & 31.6 & $33.9(93.0)$ \\
\hline Nov. 1, 2004 & 723.8 & 490.1 & 219.8 & 93.6 & 45.9 & 32.0 & 23.3 & $29.2(84.6)$ \\
\hline Dec. 6, 2004 & 866.5 & 593.6 & 279.4 & 109.8 & 50.0 & 34.8 & 28.3 & $16.2(61.2)$ \\
\hline Feb. 7, 2005 & 782.8 & 486.8 & 190.4 & 79.3 & 44.0 & 30.2 & 24.4 & $21.3(70.3)$ \\
\hline
\end{tabular}

Table 2.3.8: FWD deflections at N2 section (location according to NCAT database: station 2 inside the wheel path).

\begin{tabular}{|l|c|c|c|c|c|c|c|c|}
\hline \multicolumn{1}{|c|}{ Date } & $\begin{array}{c}\text { D0 } \\
{[\boldsymbol{\mu m}]}\end{array}$ & $\begin{array}{c}\mathbf{D 1} \\
{[\boldsymbol{\mu \mathbf { m } ]}]}\end{array}$ & $\begin{array}{c}\mathbf{D 2} \\
{[\boldsymbol{\mu \mathbf { m } ]}}\end{array}$ & $\begin{array}{c}\mathbf{D 3} \\
{[\boldsymbol{\mu \mathbf { m } ]}]}\end{array}$ & $\begin{array}{c}\mathbf{D 4} \\
{[\boldsymbol{\mu \mathbf { m } ]}}\end{array}$ & $\begin{array}{c}\mathbf{D 5} \\
{[\boldsymbol{\mu m}]}\end{array}$ & $\begin{array}{c}\text { D6 } \\
{[\boldsymbol{\mu m}]}\end{array}$ & $\begin{array}{c}\text { Temp. } \\
{ }^{\mathbf{o}} \mathbf{C}\left({ }^{\mathbf{o}} \mathbf{F}\right)\end{array}$ \\
\hline Dec. 15, 2003 & 311.8 & 230.9 & 125.6 & 72.2 & 43.9 & 29.7 & 24.0 & $15.8(60.4)$ \\
\hline Jan. 26, 2004 & 328.0 & 255.1 & 156.0 & 93.2 & 51.4 & 32.8 & 22.9 & $10.2(50.4)$ \\
\hline Feb. 23, 2004 & 299.1 & 219.5 & 126.4 & 75.1 & 48.0 & 30.9 & 21.9 & $12.5(54.5)$ \\
\hline Mar. 22, 2004 & 489.8 & 327.8 & 159.7 & 82.8 & 50.7 & 34.7 & 27.6 & $25.8(78.4)$ \\
\hline June 14, 2004 & 632.5 & 418.9 & 177.9 & 83.9 & 48.7 & 34.4 & 26.6 & $32.8(91.0)$ \\
\hline Sep. 20, 2004 & 610.6 & 384.8 & 157.9 & 71.6 & 35.0 & 33.0 & 28.7 & $31.4(88.5)$ \\
\hline Nov. 1, 2004 & 605.2 & 377.9 & 158.7 & 71.5 & 42.1 & 30.7 & 24.7 & $28.5(83.3)$ \\
\hline Dec. 6, 2004 & 570.8 & 381.1 & 182.7 & 85.1 & 45.0 & 33.1 & 28.2 & $16.0(60.8)$ \\
\hline Jan. 10, 2005 & 572.4 & 364.5 & 173.5 & 84.5 & 45.8 & 31.9 & 26.8 & $20.2(68.4)$ \\
\hline Feb. 7, 2005 & 506.0 & 314.1 & 142.2 & 66.2 & 35.7 & 25.0 & 22.3 & $19.8(67.6)$ \\
\hline
\end{tabular}




\subsection{EMBEDDED INSTRUMENTATION}

\subsubsection{Environmental Monitoring}

Instrumentation devoted to monitoring environmental changes included moisture probes and temperature gauges. Campbell Scientific moisture probes (model CS615) were installed at NCAT during the phase I experiment (Freeman et al., 2001). This type of gauge was also selected to be installed in the 'structural study' (Timm et al., 2004). These probes indicate changes in volumetric moisture content (i.e., volume of water per unit bulk volume of soil) by detecting changes in the dielectric constant of the surrounding material. The dielectric constant of soils is a composition of the dielectric constants of its individual constituents. Solid soil particles like sand and clay have dielectric constants in the range of 2 to 4 . Water, however, has a much higher dielectric constant of about 80 . Thus, increases in the moisture content of soil can be identified by measured increases in the soil's dielectric constant. The CS615 probes, sometimes referred to as water content reflectometers, use time-domain measurement methods. They consist of two parallel stainless steel rods, spaced 2 in. $(51 \mathrm{~mm})$ apart, connected to a printed circuit board which is encapsulated in epoxy. Each rod is $12 \mathrm{in.} \mathrm{(305} \mathrm{mm)}$ long and 1/8 in. $(3.2 \mathrm{~mm})$ in diameter. The circuit board transmits electrical waves that travel along the rods with travel times that depend primarily on the dielectric constant of the surrounding material. When such probes are calibrated for a specific soil, their accuracy is typically $\pm 2 \%$ moisture by volume. The readings from these probes were not considered reliable enough for the 'structural study' (personal communication, D. H. Timm, 2007).

Temperatures were monitored using passive elements based on thermistor technology (Model 108 temperature probes manufactured by Campbell Scientific, Inc.). A thermistor is essentially a resistor whose resistance varies according to temperature. For each test section in the 'structural study,' four thermistors were bundled together to provide temperature information near the surface and at the following depths: 2, 4 and 10 in. (51, 102 and $254 \mathrm{~mm}$ ). These probes were installed after paving had been completed given their survival temperature range is $-50^{\circ} \mathrm{C}$ to $+100^{\circ} \mathrm{C}\left(-58^{\circ} \mathrm{F}\right.$ to $\left.212^{\circ} \mathrm{F}\right)$. Over the range of $-3^{\circ} \mathrm{C}$ to $90^{\circ} \mathrm{C}\left(26.6^{\circ} \mathrm{F}\right.$ to $\left.194^{\circ} \mathrm{F}\right)$ the measurement accuracy of these 
probes is $\pm 0.3^{\circ} \mathrm{C}\left( \pm 0.54^{\circ} \mathrm{F}\right)$. Unlike the moisture probes, the temperature probes do not need to be calibrated for the particular environment in which they are to be used. In Freeman et al. (2001) they were tested and found repeatable and accurate.

\subsubsection{Mechanical Responses}

Mechanical responses at the 'structural study' were measured with strain gauges and pressure cells. Both device types are considered suitable for measuring dynamic responses only because they experience drift over time (for various reasons) that precludes their use in monitoring permanent changes. In each section an array of 12 stain gauges was attached to the bottom of the HMA course at a depth of 5 in. (i.e., $\mathrm{z}=127 \mathrm{~mm}$ ). The asphalt strain gauges were manufactured by Construction Technologies Laboratories (CTL Group) Model ASG-152. A picture of one such a gauge with corresponding dimensions (in inches) is shown in Figure 2.4.1. It may be seen that the gauge is made of two ' $T$ ' shaped metal elements interconnected by a $2 \mathrm{in}$. long (51 mm) measuring sensor.
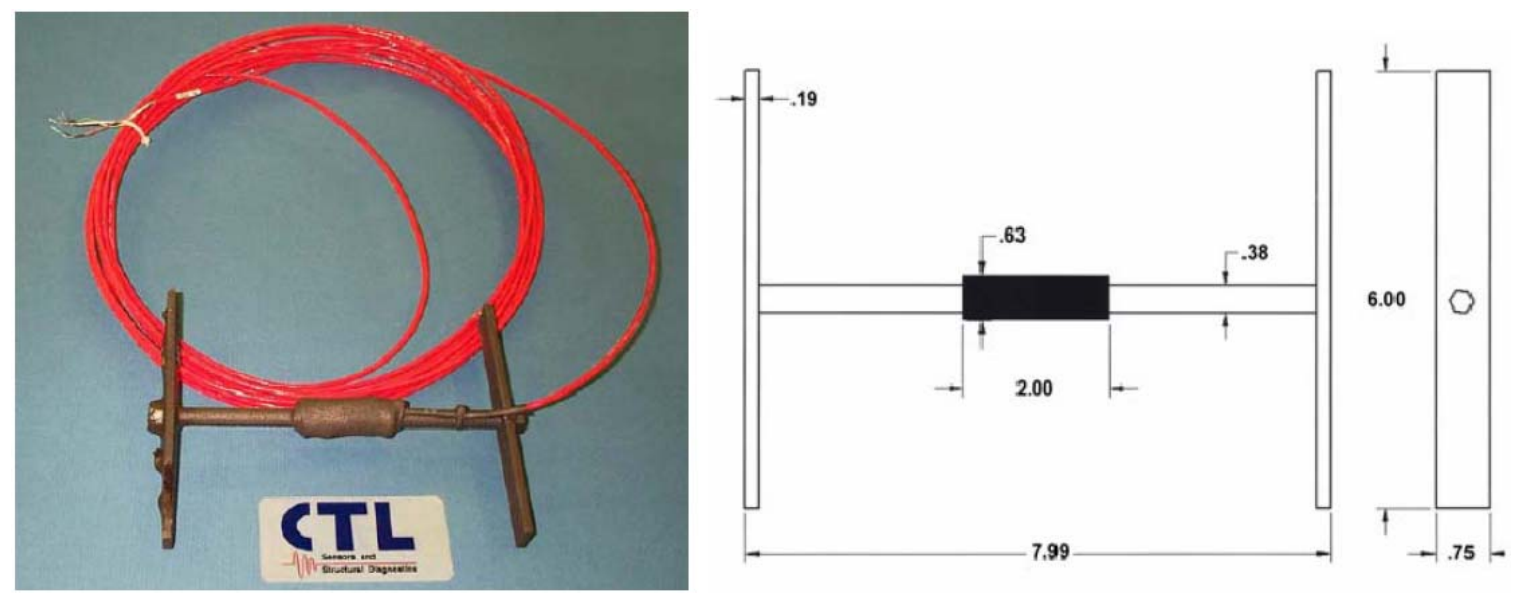

Figure 2.4.1: Photograph and dimensions (in inches) of an asphalt strain gauge (Timm et al. 2004).

Two pressures cells were embedded within the pavement system: one on top of the base course (i.e., $\mathrm{z}=5$ in. or $\mathrm{z}=127 \mathrm{~mm}$ ) and another on top of the subgrade ( $\mathrm{z}=11 \mathrm{in}$. or $\mathrm{z}=280 \mathrm{~mm}$ ). Both pressure cells were manufactured by Geokon (3500 circular model). These devices (see Figure 2.4.2) are constructed from two slightly convex stainless steel plates welded together around their periphery and separated by a narrow 
gap filled with de-aired hydraulic fluid. When external pressure is applied to the plates, the two plates are squeezed together causing a corresponding increase of fluid pressure inside the cell. High pressure stainless steel tubing connects the pressure cell to a semiconductor pressure transducer which converts the increased pressure of the compressed fluid into an electrical signal. This signal is transmitted through a signal cable to the readout location.

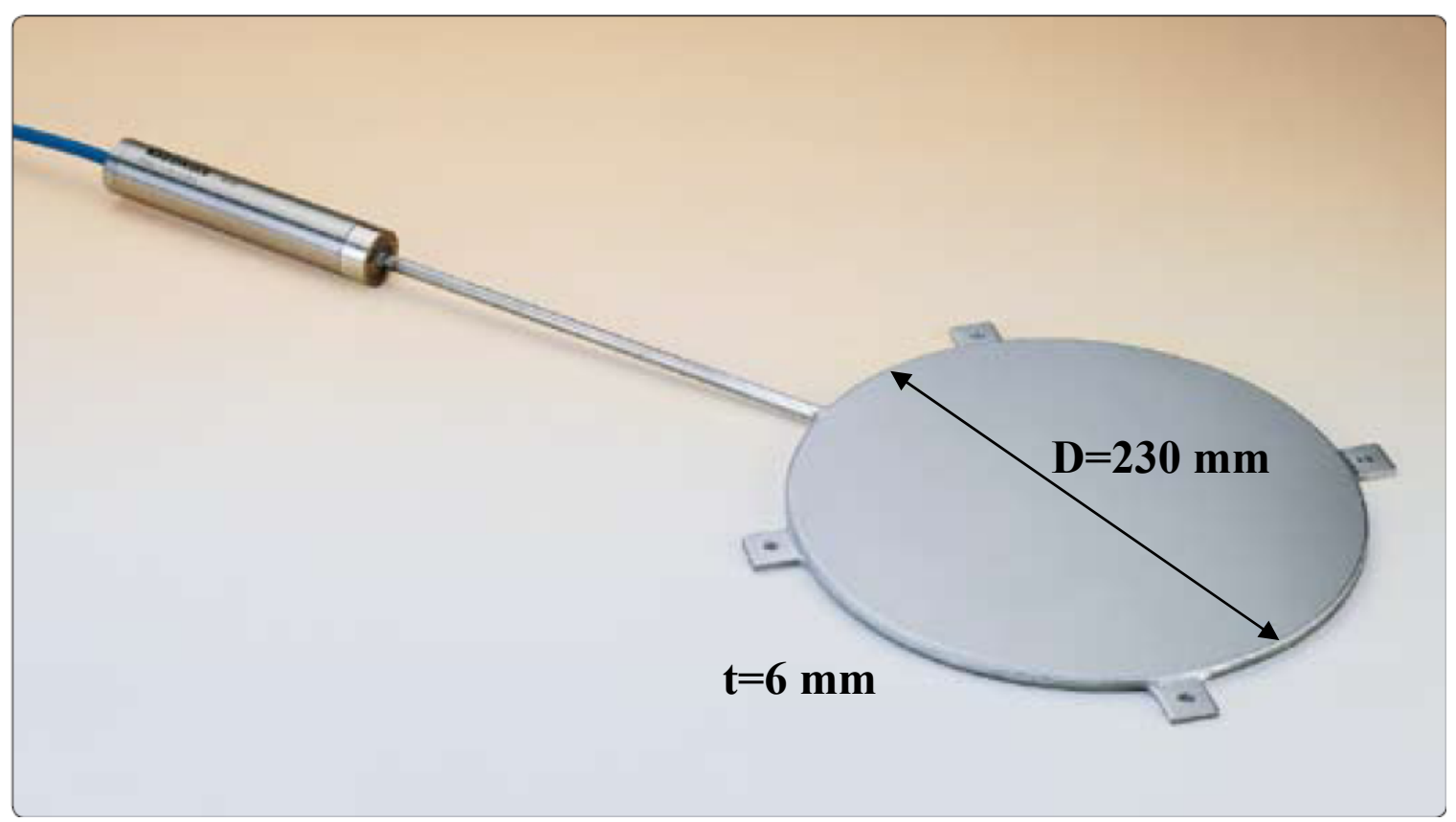

Figure 2.4.2: Photograph of Geokon Earth Pressure cell Model 3500.

The instrumentation layout shown in Figure 2.4.3 refers to Section N1. An essentially identical layout was assembled in Section N2. The center point of this array was positioned along the right wheel path, about $8 \mathrm{ft}$ ( $2.44 \mathrm{~m}$ ) away from the centerline. The $\mathrm{Y}$-axis in the figure points in the direction of truck travel while the $\mathrm{X}$-axis points in the transverse direction. The array of 12 asphalt strain gauges can be seen in the figure, spaced evenly 24 in. (610 mm) apart in both $\mathrm{X}$ and $\mathrm{Y}$ directions.

Strain gauges BLL, BLC, BLR, ALL, ALC, and ALR are all measuring strains in Y. The rest of the strain gauges, namely BTL, BTC, BTR, ATL, ATC and ATR, are measuring strains in $\mathrm{X}$. It should be noted that five of the strain gauges, ALL, ATL, BLL, ATR and BTR, did not survive the construction process. The two pressure cells are also shown in the figure as BBC and ASC. The BBC gauge was located on top of 
the base course and under the HMA course ( $\mathrm{z}=5$ in. or $\mathrm{z}=127 \mathrm{~mm}$ ). It was designated to capture vertical stresses (i.e., stress in Z) at this interface. This gauge had a $36.3 \mathrm{psi}$ (0.25 MPa) range. The ASC gauge was designated to measure the vertical stresses at the interface between the subgrade and the granular base $(\mathrm{z}=11$ in. or $\mathrm{z}=280 \mathrm{~mm}$ ). This gauge had a $14.5 \mathrm{psi}(0.1 \mathrm{MPa})$ range given that it is placed at a greater depth.

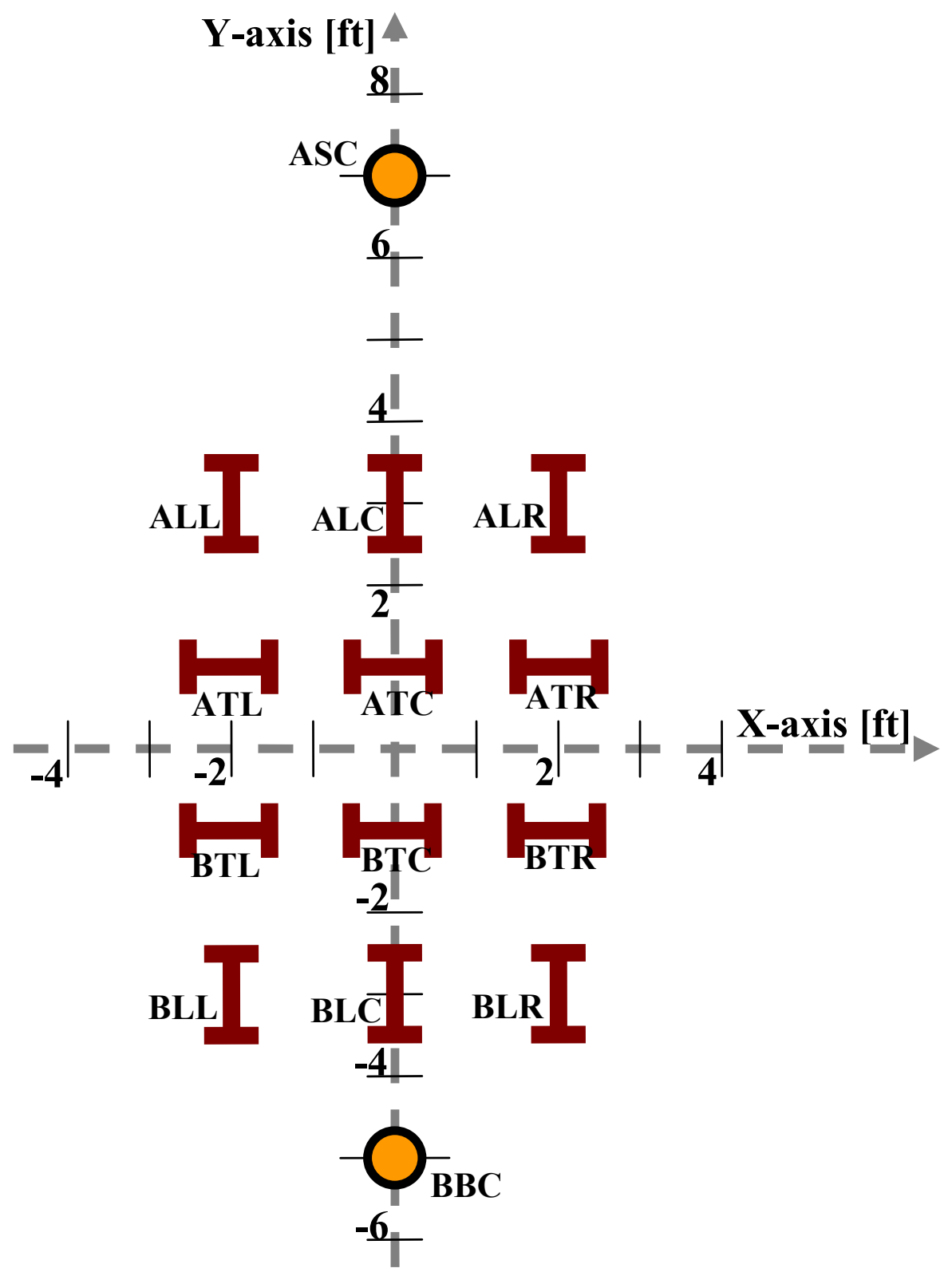

Figure 2.4.3: Sensor layout for section N1 (based on Timm et al., 2004). 


\subsection{STRUCTURAL BEHAVIOR AT NCAT}

\subsubsection{Resilient Response}

The resilient responses presented hereafter were obtained from Section N1 while in its pristine state. The data was collected soon after construction, on November 7, 2003, during which a single NCAT truck was doing multiple laps. The corresponding raw data file provided by NCAT for the purpose of this study contained three such laps (see Appendix A). Herein, the responses due to the first of the three passes is presented and discussed.

Each time the truck approached the gauge array, the data acquisition system was switched on and the gauge readings were recorded for a period of about 2 seconds. Between truck passes, for a period of about 2 minutes, the data acquisition system was switched off in order to save storage space. The pavement temperatures in different depths during this experiment are reported in Table 2.5.1. The values in the table are hourly averages, based on minute-by-minute readings, corresponding to the hour in which the data files were created (personal communication, D. H. Timm, 2007).

Table 2.5.1: Temperature profile in Section N1 for analysis of resilient response data.

\begin{tabular}{|c|c|}
\hline Depth, in. (mm) & Temperature, ${ }^{\mathbf{}} \mathbf{F}\left({ }^{\mathbf{}} \mathbf{C}\right)$ \\
\hline 0 & $88.5(31.4)$ \\
\hline $2(51)$ & $80.9(27.2)$ \\
\hline $4(102)$ & $77.1(25.1)$ \\
\hline $10(254)$ & $73.7(23.2)$ \\
\hline
\end{tabular}

The following figures present the measured N1 responses to one truck pass as recorded by the gauge array layout shown in Figure 2.4.3. In all figures the abscissa represents test time and ranges between 0 and 2.2 seconds (the zero is arbitrary). In each case circular markers denote NCAT data; the solid line passing between the data points was created using cubic spline interpolation as means of increasing the 'measurement' density (this increased measurement density will be used later on in the report). It is possible to identify and relate graphically the data in the charts to the specific axle. This is done using the terminology in Table 2.4.1 where ' 1 ' ' refers to the steering axle, ' $1 \mathrm{D}$ ' 
and ' $2 \mathrm{D}$ ' refer to the drive axle and ' $1 \mathrm{~T}$ ' to ' $5 \mathrm{~T}$ ' refer to the trailer axles. When reviewing the charts it should be borne in mind that the location of each axle relative to the gauges was not measured. In fact, the tractor unit and trailers did not follow a straight line and did not move along or parallel to the Y-axis in Figure 2.4.3. Mainly for this reason, axles having similar weight recorded different peak responses and sometimes even responses of opposite sign (compare response due to axles 1T, 2T, 3T and $4 \mathrm{~T}$ with the response due to axle $5 \mathrm{~T}$ in Figure 2.5.4).

Figure 2.51 includes two charts, both presenting measured vertical stresses (i.e., stress in Z). The upper chart shows readings from gauge placed on top of the aggregate base (i.e., gauge BBC) and the lower chart shows readings from the gauge placed on top of the subgrade (i.e., gauge ASC). The ordinate in both charts ranges between 0 and 18 psi. In both cases it is easy to identify the individual axles. As expected, peak magnitudes are generally lower in the bottom chart. Also it can be seen that, excluding the drive axle (dual-tandem), the pressure cells recover fully, i.e., return to a zero reading, in between individual axle passes. Also interesting to note is that the speed of the truck can be calculated using the figures. For example, the peak stress due to the steer axle occurs at $\mathrm{t}=0.395$ seconds according to gauge ASC and at $\mathrm{t}=0.213$ seconds in gauge BBC. From Figure 2.4.3 it can be seen that these gauges are spaced 12 feet apart. Therefore, the NCAT truck covered a distance of 12 feet in 0.182 seconds. The calculated truck speed is 65.93 foot per second or $44.95 \mathrm{mph}$ which is extremely close to the target speed of $45 \mathrm{mph}$.

Figure 2.5.2 includes two charts, both presenting strains measured at the bottom of the HMA course in the direction loading (i.e., strain in Y) by gauges ALC and BLC. Both gauges are located on the Y-axis in Figure 2.4.3. Similarly, Figure 2.5.3 presents the strains in Y measured at the bottom of the HMA course by gauges ALR and BLR. Recall that the location of these gauges is offset by $24 \mathrm{in}$. (610 mm) compared to the $\mathrm{Y}$ axis in Figure 2.4.3. The ordinate in both cases ranges from -400 microstrains to +200 microstrains. As can be seen, strain reversal is induced to the HMA course as the axle wheels travel over the pavement. As the load is approaching the gauges measure compressive strains. Tensile strains are induced as the load gets closer to the gauge. The strains go back into compression as the load is receding. 

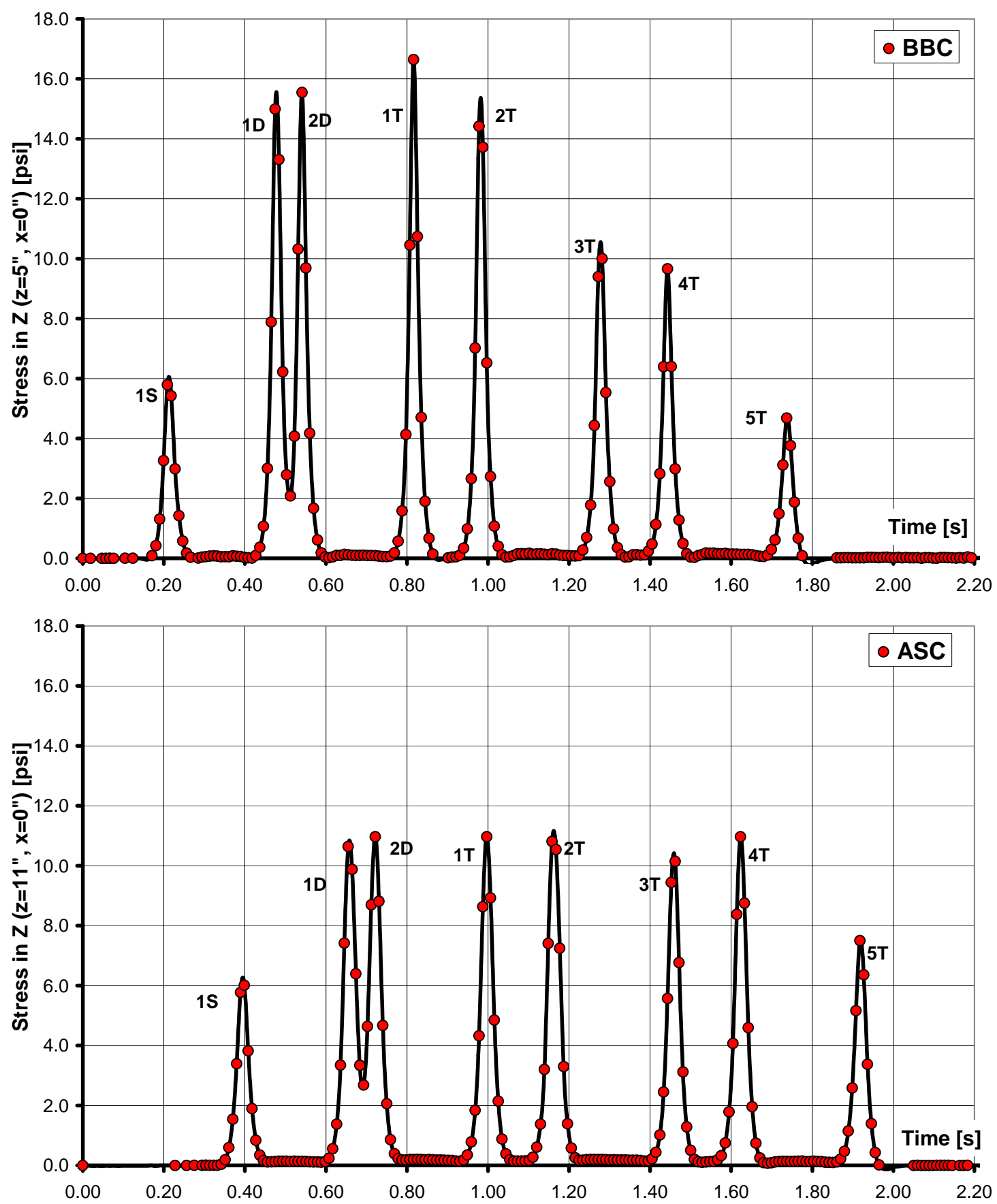

Figure 2.5.1: Vertical stresses (i.e. stress in Z) on top of the base course (upper chart) and on top of the subgrade (lower chart) as a result of one truck pass. Gauges positioned along the $\mathrm{Y}$-axis in Figure 2.4.3. 

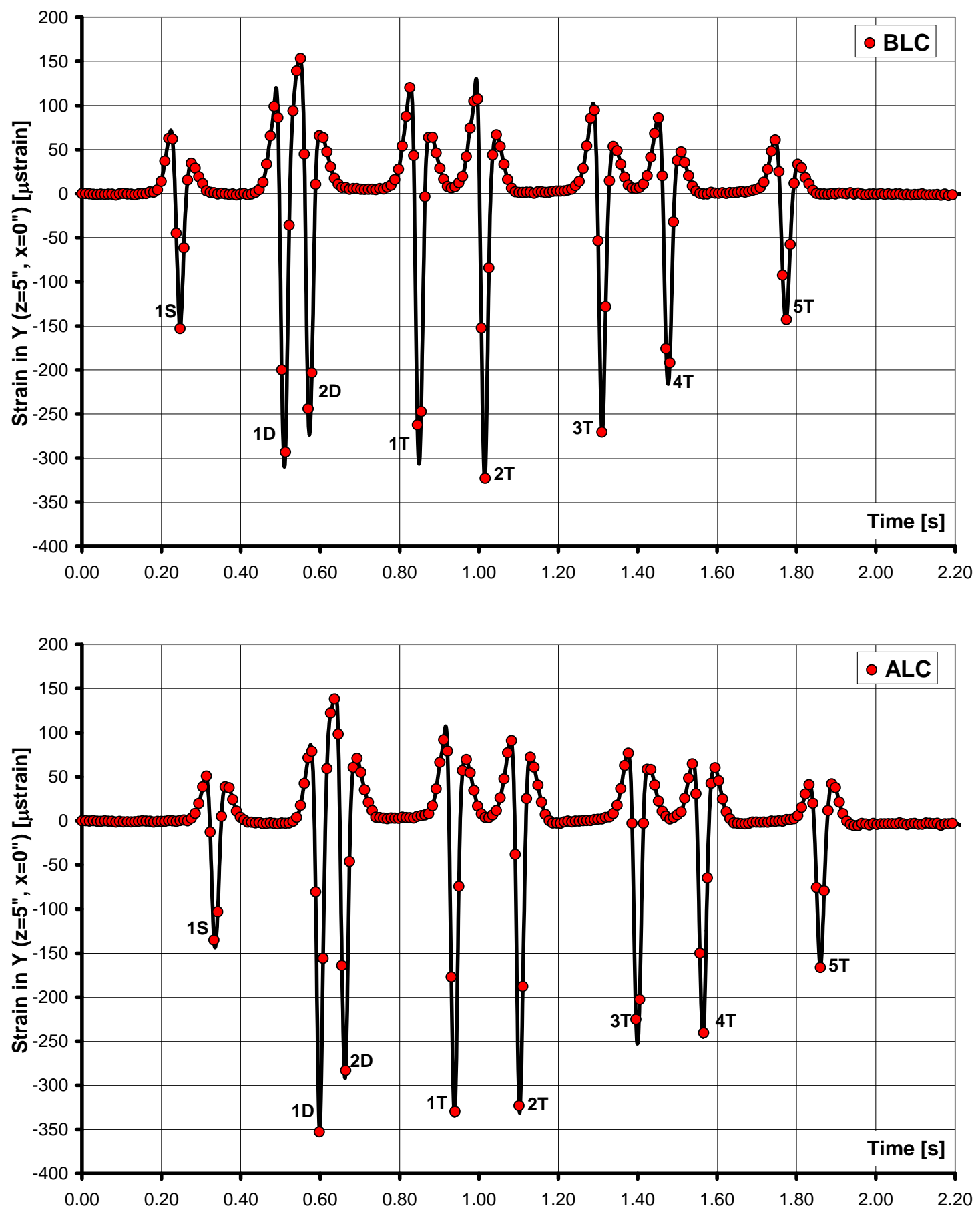

Figure 2.5.2: Horizontal strains in the loading direction (i.e., strain in Y) at the bottom of the HMA course as a result of one truck pass. Gauges positioned along the Y-axis in Figure 2.4.3. 

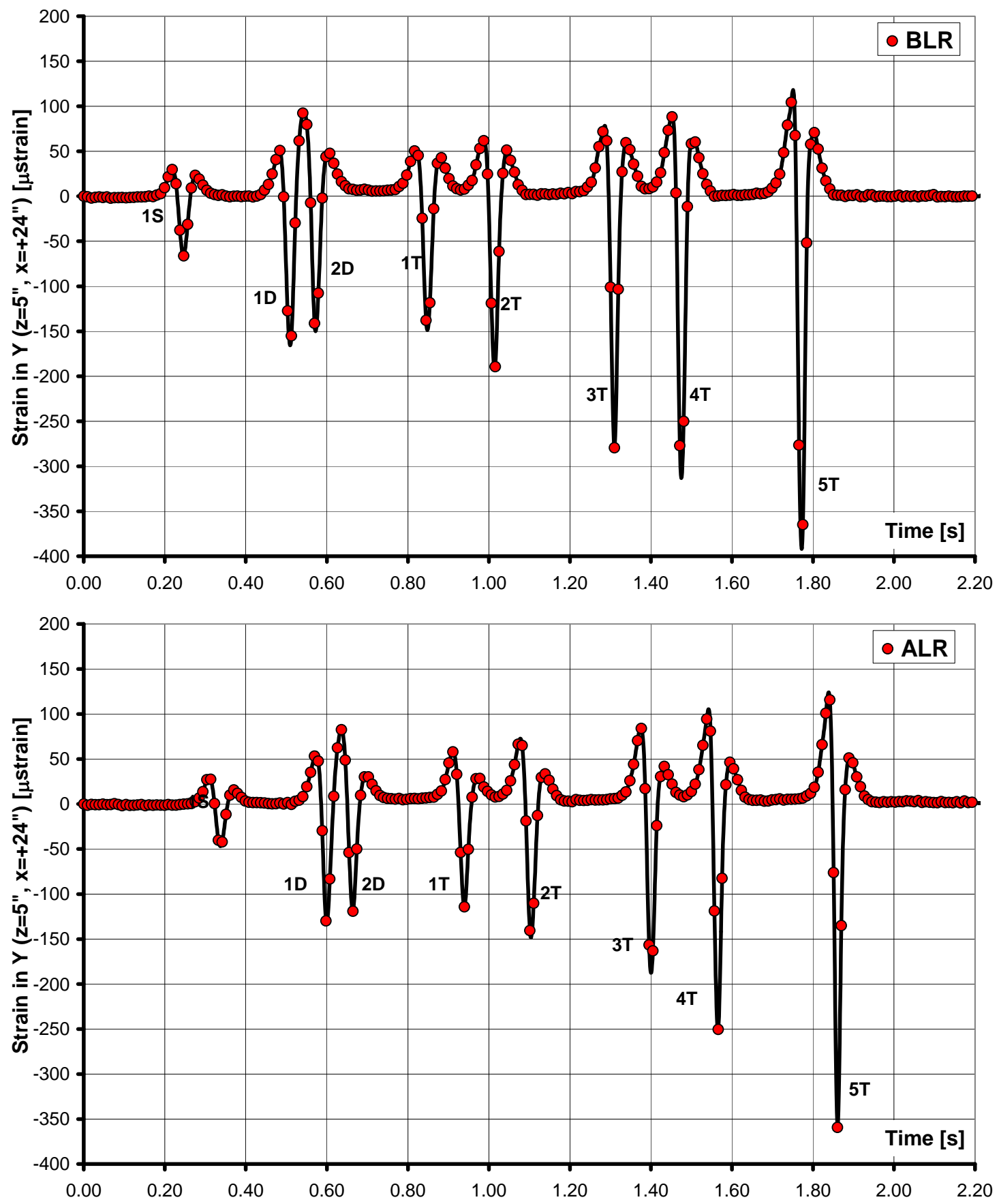

Figure 2.5.3: Horizontal strains in the loading direction (i.e., strain in Y) at the bottom of the HMA course as a result of one truck pass. Gauges offset by 24 in. $(610 \mathrm{~mm})$ compared to the Y-axis in Figure 2.4.3.

The final two charts in Figure 2.5.4 present horizontal strains at the bottom of the HMA course (i.e., $z=5$ in. or $z=127 \mathrm{~mm}$ ) as measured by gauges ATC and BTC in Figure 2.4.3 (i.e., strain in X). As can be seen, tensile (negative) strains of up to 400 
microstrains in magnitude were induced in the transverse direction by all axles except for the last one (axle 5T). As mentioned earlier, the reason for this change in sign is related to the location of the load relative to the gauge.
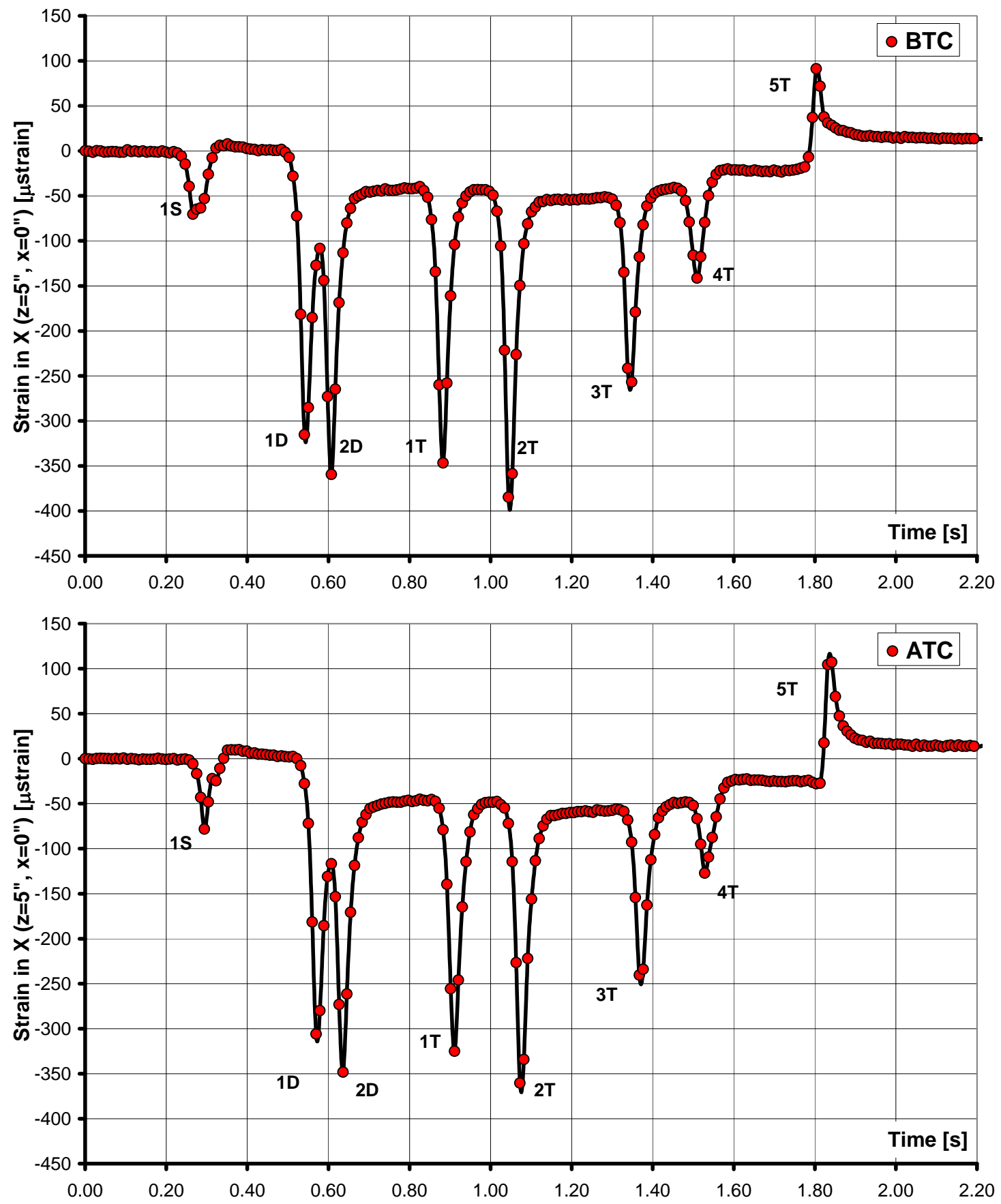

Figure 2.5.4: Horizontal strains in the transverse direction (i.e., strain in $\mathrm{X}$ ) at the bottom of the HMA course as a result of one truck pass. Gauges positioned along Yaxis in Figure 2.4.3. 


\subsubsection{Cracking and Rutting Performance}

Sections N1 and N2 experienced cracking in a very similar manner (Priest and Timm, 2006; Timm et al., 2006). Both failed in fatigue within two months of each other (see Figure 2.5.5. Section N1 (modified HMA) failed prior to section N2 (unmodified HMA) after six months of traffic. First, small transverse cracks appeared in the wheel path. Then the cracks progressed to the edge of the wheel path and often curled in the direction of traffic. Later, the individual transverse cracks became interconnected into a classical alligator pattern. Pumping of the fines from the unbound aggregate base through the cracks was also observed in the individual transverse cracks as well as the alligator cracked areas. The progression of fatigue failure was fairly rapid once the first cracks appeared and especially once pumping began. Subsequently, the responses quickly over ranged the embedded instrumentation. More detailed crack mapping can be found in Priest and Timm (2006).

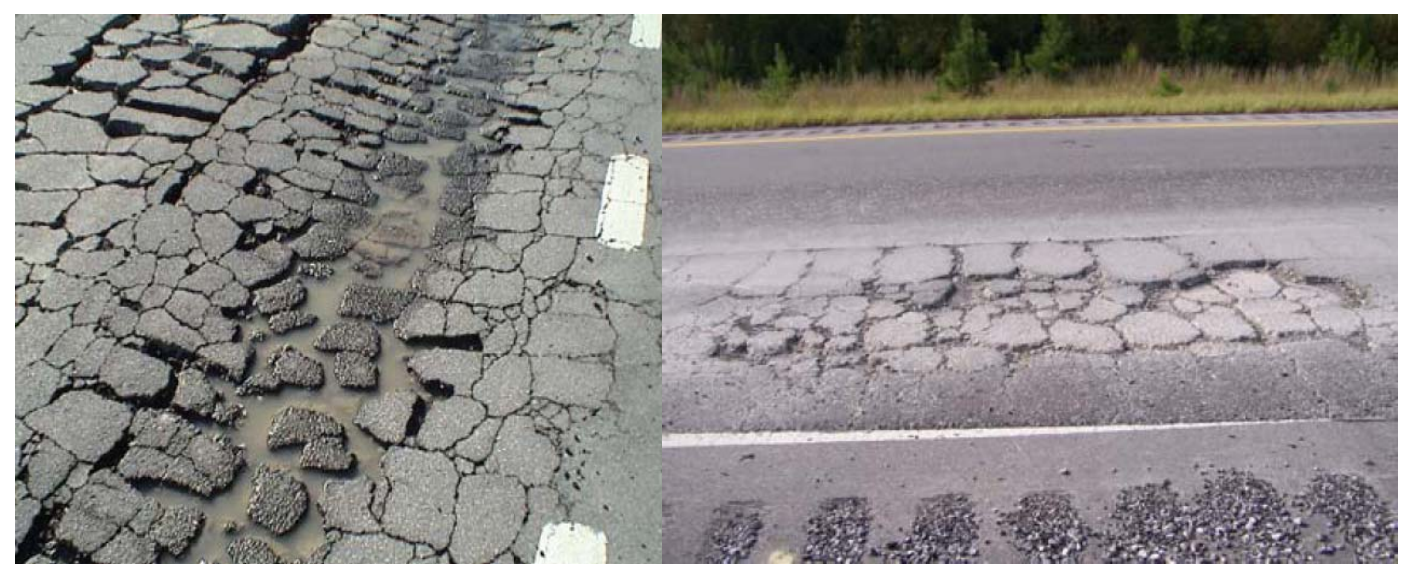

Figure 2.5.5: Fatigued sections N1 (left photo) and N2 (right photo).

With respect to rutting, sections N1 and N2 did not rut much by the time they failed in fatigue. The progression of average rut depth is shown in Table 2.5 .2 (personal communication, B. Powell, 2007); this data is also presented graphically in Figure 2.5.6. As can be seen, after the application of about 4.5 million equivalent single axle loads (ESALs), the final average rut depth was only about $8 \mathrm{~mm}$. 
Table 2.5.2: Tabulated progression of N1 and N2 rutting levels vs. number of applied ESALs.

\begin{tabular}{|c|c|c|c|c|}
\hline$\#$ & $\begin{array}{c}\text { Cumulative } \\
\text { ESALs }\end{array}$ & $\begin{array}{c}\text { Measurement } \\
\text { Date }\end{array}$ & $\begin{array}{c}\text { Rutting in } \\
\text { N1 mm, (in.) }\end{array}$ & $\begin{array}{c}\text { Rutting in } \\
\mathrm{N} 2, \mathrm{~mm} \text { (in.) }\end{array}$ \\
\hline 1 & 0 & $9 / 12 / 03$ & $0.00(0.00)$ & $0.00(0.00)$ \\
\hline 2 & 10,670 & $10 / 27 / 03$ & $0.21(0.01)$ & $0.30(0.01)$ \\
\hline 3 & 40,068 & $11 / 10 / 03$ & $0.27(0.01)$ & $0.35(0.01)$ \\
\hline 4 & 218,357 & $12 / 8 / 03$ & $0.27(0.01)$ & $0.42(0.02)$ \\
\hline 5 & 643,101 & $1 / 12 / 04$ & $0.65(0.03)$ & $0.71(0.03)$ \\
\hline 6 & 727,772 & $1 / 19 / 04$ & $0.33(0.01)$ & $0.43(0.02)$ \\
\hline 7 & $1,055,293$ & $2 / 16 / 04$ & $0.65(0.03)$ & $0.85(0.03)$ \\
\hline 8 & $1,115,778$ & $2 / 23 / 04$ & $0.68(0.03)$ & $0.85(0.03)$ \\
\hline 9 & $1,179,894$ & $3 / 1 / 04$ & $0.68(0.03)$ & $0.84(0.03)$ \\
\hline 10 & $1,259,225$ & $3 / 8 / 04$ & $0.84(0.03)$ & $1.34(0.05)$ \\
\hline 11 & $1,608,513$ & $4 / 5 / 04$ & $1.05(0.04)$ & $2.03(0.08)$ \\
\hline 12 & $1,695,079$ & $4 / 12 / 04$ & $1.30(0.05)$ & $1.51(0.06)$ \\
\hline 13 & $1,787,799$ & $4 / 19 / 04$ & $1.33(0.05)$ & $1.51(0.06)$ \\
\hline 14 & $2,233,571$ & $5 / 24 / 04$ & $2.07(0.08)$ & $2.93(0.12)$ \\
\hline 15 & $2,412,440$ & $6 / 7 / 04$ & $2.78(0.11)$ & $3.54(0.14)$ \\
\hline 16 & $2,501,549$ & $6 / 14 / 04$ & $2.84(0.11)$ & $3.49(0.14)$ \\
\hline 17 & $2,595,810$ & $6 / 21 / 04$ & $2.98(0.12)$ & $3.78(0.15)$ \\
\hline 18 & $2,694,521$ & $6 / 28 / 04$ & $3.07(0.12)$ & $3.92(0.15)$ \\
\hline 19 & $2,863,433$ & $7 / 12 / 04$ & $3.43(0.14)$ & $4.31(0.17)$ \\
\hline 20 & $2,970,955$ & $7 / 19 / 04$ & $3.86(0.15)$ & $4.87(0.19)$ \\
\hline 21 & $3,060,410$ & $7 / 26 / 04$ & $4.10(0.16)$ & $5.08(0.20)$ \\
\hline 22 & $3,166,906$ & $8 / 2 / 04$ & $4.28(0.17)$ & $5.11(0.20)$ \\
\hline 23 & $3,450,232$ & $8 / 23 / 04$ & $4.32(0.17)$ & $5.21(0.21)$ \\
\hline 24 & $3,559,500$ & $8 / 30 / 04$ & $4.14(0.16)$ & $5.59(0.22)$ \\
\hline 25 & $3,741,860$ & $9 / 13 / 04$ & $4.91(0.19)$ & $6.05(0.24)$ \\
\hline 26 & $3,921,345$ & $9 / 26 / 04$ & $5.02(0.20)$ & $6.18(0.24)$ \\
\hline 27 & $4,031,786$ & $10 / 4 / 04$ & $5.36(0.21)$ & $6.44(0.25)$ \\
\hline 28 & $4,122,945$ & $10 / 11 / 04$ & $5.84(0.23)$ & $6.11(0.24)$ \\
\hline 29 & $4,219,483$ & $10 / 18 / 04$ & $5.68(0.22)$ & $6.95(0.27)$ \\
\hline 30 & $4,300,286$ & $10 / 25 / 04$ & $6.70(0.26)$ & $7.21(0.28)$ \\
\hline 31 & $4,382,730$ & $11 / 1 / 04$ & $8.04(0.32)$ & $7.52(0.30)$ \\
\hline 32 & $4,553,790$ & $11 / 15 / 04$ & $7.97(0.31)$ & $7.99(0.31)$ \\
\hline 33 & $4,671,075$ & $11 / 29 / 04$ & $7.64(0.30)$ & $8.32(0.33)$ \\
\hline
\end{tabular}




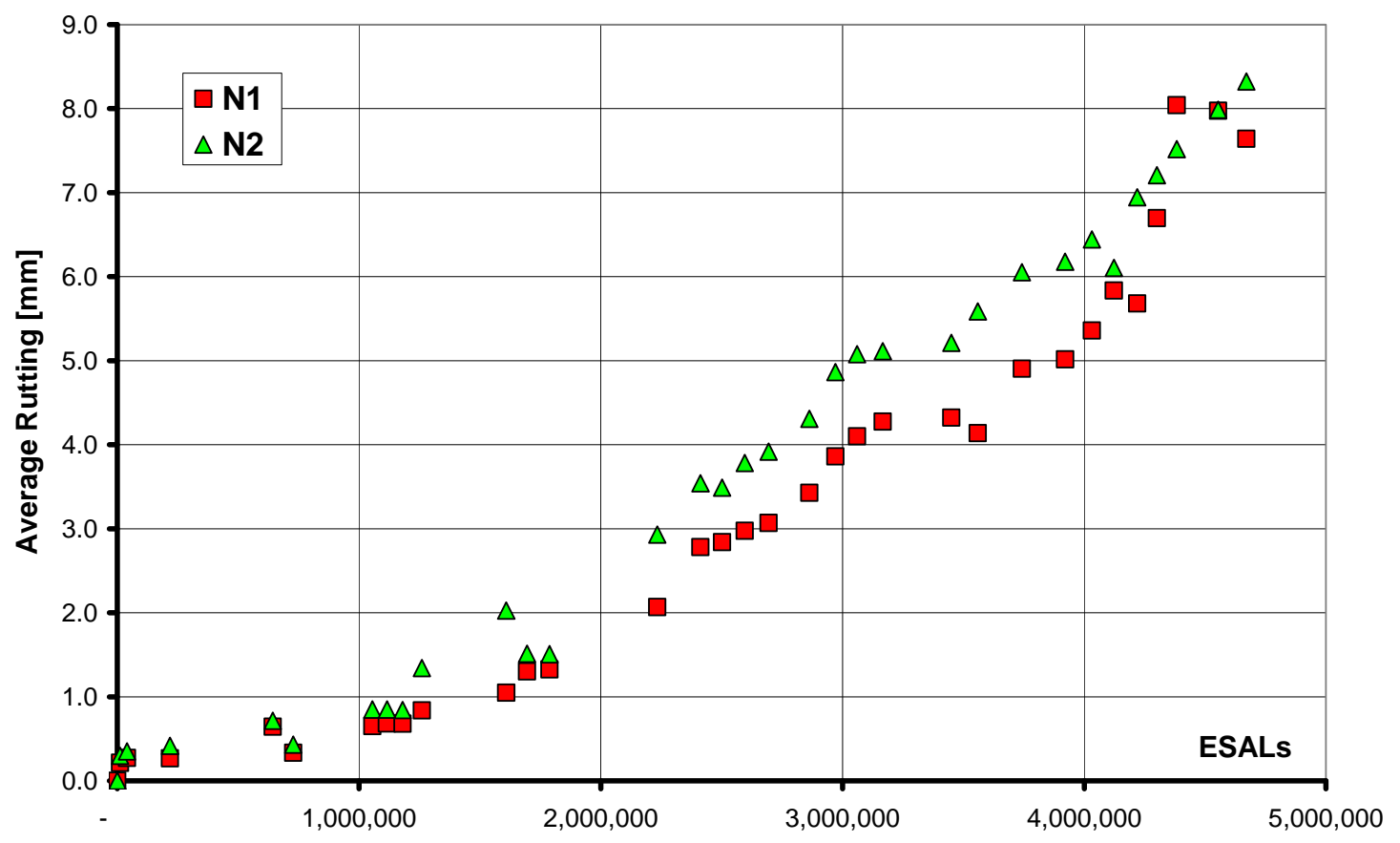

Figure 2.5.6: Graphical progression of N1 and N2 rutting levels vs. number of applied ESALs at the Track. 


\section{CHAPTER 3 - THE APT EXPERIMENT}

This chapter summarizes the APT work conducted jointly by INDOT and Purdue University through the North Central Superpave Center between the years 2004 and 2006. It describes the loading history and environment prevailing during the APT experiment and some preliminary analyses of pertinent test data. Here, a subset of the available data is identified as suitable and sufficient for carrying out the main study objective. Finally, to emphasize the need for fundamental analysis, as performed in the following chapters, a direct comparison with the NCAT results is provided.

\subsection{FACILITY DESCRIPTION}

The INDOT APT facility was fabricated in the early 1990's; it was designed and constructed by Purdue University under a joint venture between INDOT and the School of Civil Engineering (White et al., 1990; Galal et al., 1998). The facility is housed in a 2,000 sq. ft ( 186 sq. m) hangar located near INDOT’s Office of Research and Development in West Lafayette. The hangar is divided into three major areas, a testing area, a utility area and an operator control area. Figure 3.1.1 shows a schematic floor plan of the facility, in which the three different areas can be seen.

The testing area consists of a test pit embedded in a concrete floor. It is $6 \mathrm{ft}$ deep $(1.83 \mathrm{~m})$ and shaped as a square with $20 \mathrm{ft}(6.1 \mathrm{~m})$ long sides. Prototype pavements for testing are constructed inside this pit. Typically, different structures are constructed in the pit, each $20 \mathrm{ft}(6.1 \mathrm{~m})$ long. The width of each section depends on the experimental configuration. The $\mathrm{n} 1$ and $\mathrm{n} 2$ sections addressed herein were each $10 \mathrm{ft}$ (3.05 m) wide; accordingly, as can be seen in Figure 3.1.1 the test pit is split into two lanes (1 and 2). The NCAT N1 section was replicated in Lane 1 and the N2 section was replicated in Lane 2. Additionally, since the pit is surrounded by concrete from all sides and bottom, water can be introduced into the subgrade. Overhanging the test pit from the ceiling is a radiant heating system. The purpose of this system is to stabilize and control the air temperature during testing; it is capable of heating the air in the test area up to $140^{\circ} \mathrm{F}$ $\left(60^{\circ} \mathrm{C}\right.$ ), and maintaining constant pavement surface temperatures to within $\pm 2^{\circ} \mathrm{F}$ (if all doors remain closed). Additionally, the facility has some cooling capabilities using an 
air-conditioning unit. In its maximum capacity, the air temperature inside the test area can be reduced relative to warmer outdoor conditions to $60^{\circ} \mathrm{F}\left(15.5^{\circ} \mathrm{C}\right)$. In this study the temperature in the testing area was set to $60^{\circ} \mathrm{F}\left(15.5^{\circ} \mathrm{C}\right)$. A humidity detector is positioned close to the test pit; in principle, it is possible to increase the humidity in the test area by intentionally ponding water.

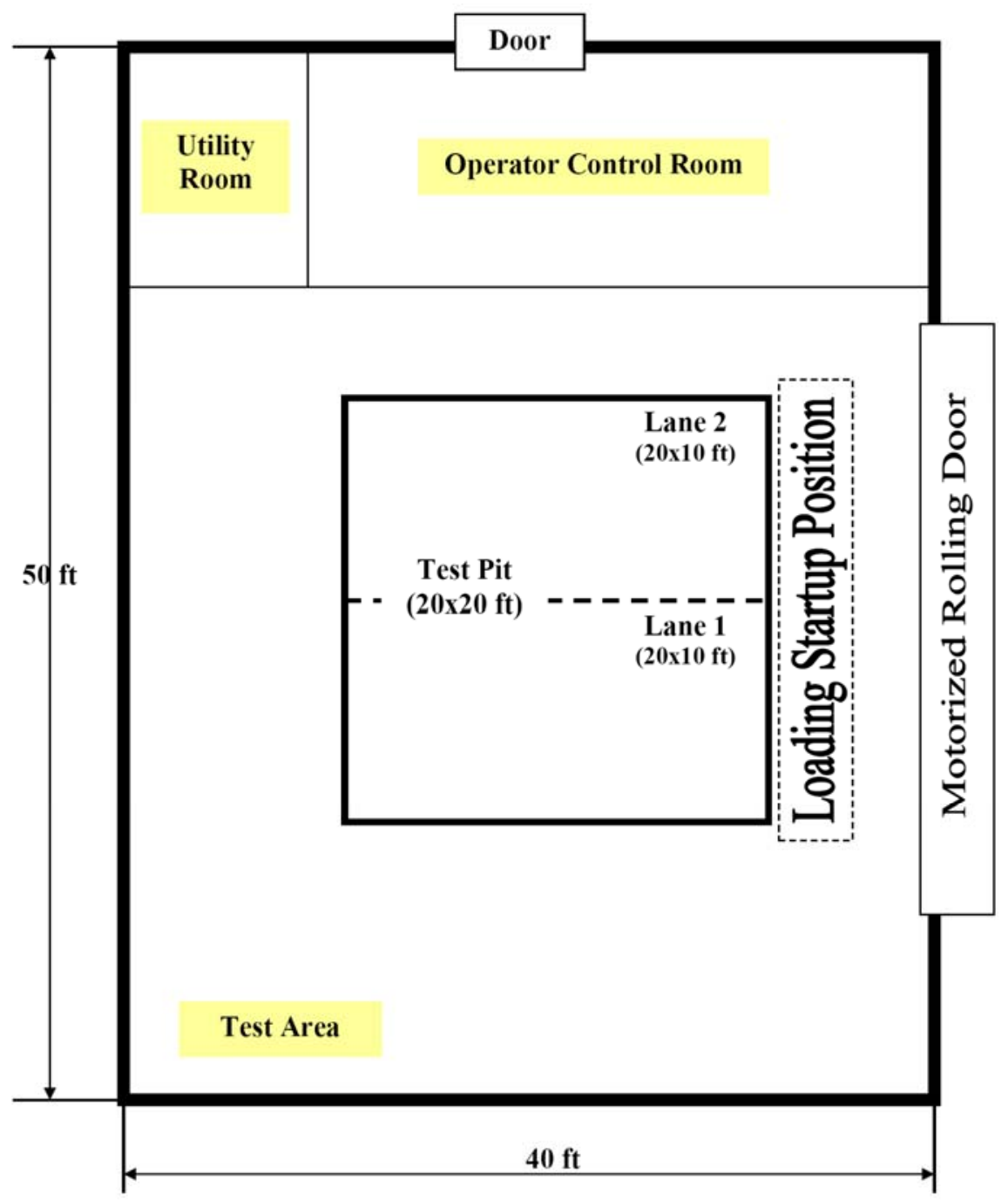

Figure 3.1.1: Schematic floor plan of INDOT APT facility.

The APT loading system is mounted on a large steel frame with beams spanning across and bridging the test pit. The frame itself is fixed on each side to steel rails embedded in the concrete floor. The fixture can be loosened to allow positioning of the frame above any test-lane of choice. The loading system is designed to produce a downward force of up to $20,000 \mathrm{lb}(9,080 \mathrm{~kg})$. This force is produced by four 
interconnected pneumatic cylinders. A precision air pressure gauge is used to adjust and control the magnitude of the force throughout the test. The downward force is applied to the pavement surface through a wheel assembly. Two tire assembly types are available: dual/conventional and single wide-base. The tire inflation pressures in each case are adjustable, up to a maximum of $120 \mathrm{psi}(0.84 \mathrm{MPa})$. Either assembly is mounted on a carriage capable of traversing the test pit by traveling on the steel beams. The carriage is cable driven by a motor and a control drive. This motor was designed to accelerate the carriage (with wheel assembly) from a static startup position to a speed of $5 \mathrm{mph}$ (8 $\mathrm{km} / \mathrm{h}$ or $2.235 \mathrm{~m} / \mathrm{s})$ within the first $5 \mathrm{ft}(1.52 \mathrm{~m})$ of the test pit. The carriage speed is then maintained constant, at $5 \mathrm{mph}(8 \mathrm{~km} / \mathrm{h})$, for the next $12 \mathrm{ft}(3.66 \mathrm{~m})$. Finally, the speed is reduced back to zero within the last $3 \mathrm{ft}(0.91 \mathrm{~m})$ of the test pit. For this study passes were applied via the dual-wheel assembly loaded to $15,000 \mathrm{lb}(6,810 \mathrm{~kg})$ with tires inflated to $100 \mathrm{psi}(0.70 \mathrm{MPa})$. Figure 3.1.2 shows a picture of the APT loading system (the test pit is empty in this picture).

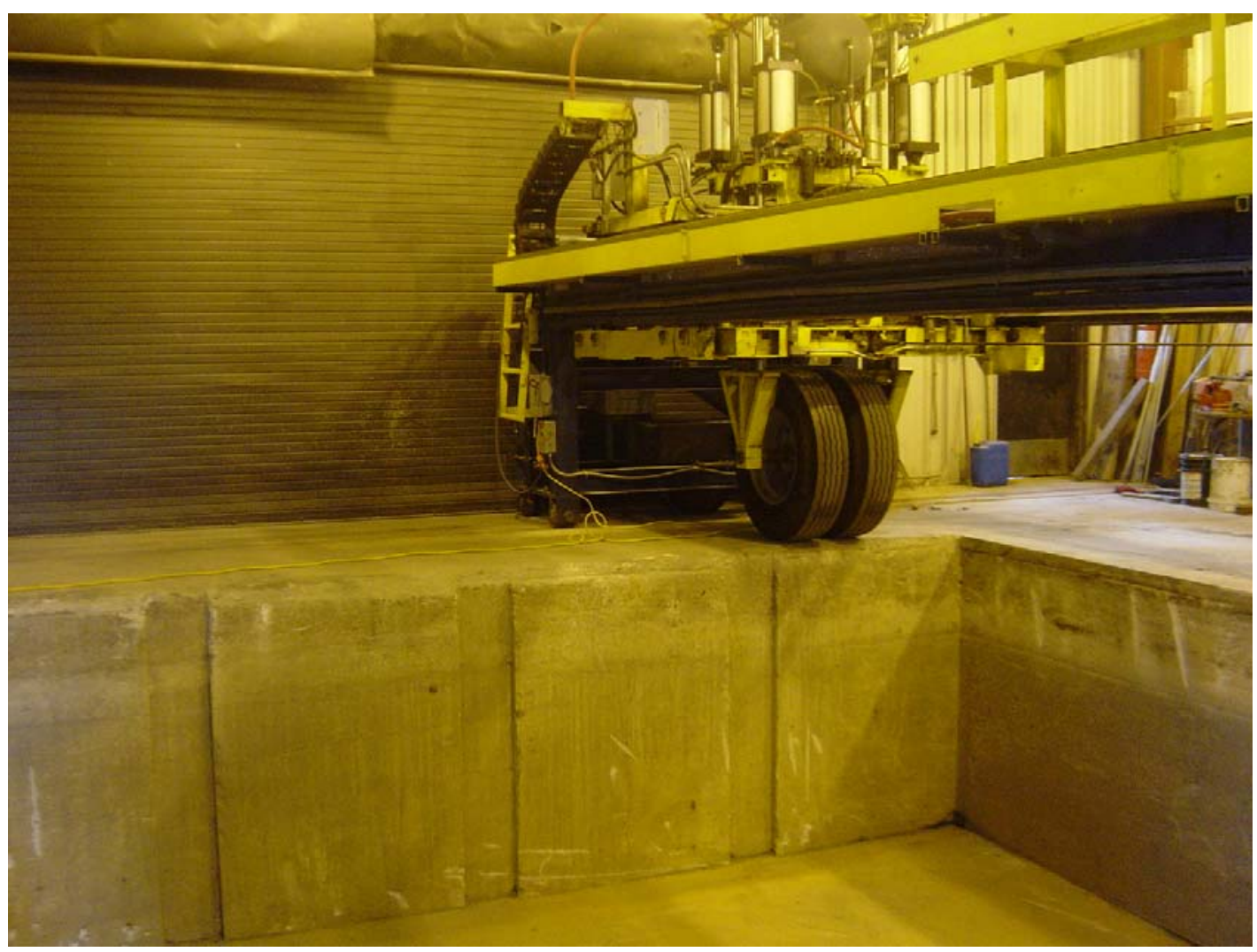

Figure 3.1.2: Picture of empty test pit and APT loading system. 
Depending on the desired mode of application, the wheel assembly can be raised from the ground by reversing the action of the four pneumatic cylinders and returned to the startup position for another loading cycle; this will result in a unidirectional mode of loading. Alternatively, the pavement can also be loaded while the carriage travels back to the startup position; this will result in a bidirectional mode of loading. Moreover, trafficking in the APT can be applied repeatedly in the same wheel path or with wander. In the latter case, the wheel path is randomly selected by a computer to within \pm 5 in $( \pm 125 \mathrm{~mm}$ ) from the centerline. The transverse movement for producing wander is governed by an electric servo-motor also attached to the steel frame. Under this study APT passes were applied in unidirectional mode only. As will be discussed later (see Section 3.4), except for the initial part of the experiment passes were applied with wander.

The utility room houses a boiler, transformer, heating controls and a water circulating system. The latter can be used to introduce hot water into pipes embedded in one of the pavement layers. This feature is mostly used when testing rigid pavements or composite pavements (i.e., HMA overlaying concrete) to heat up the concrete slabs. The operator control room houses computers and an interface to fully interact with and control the APT operation. Currently this room is equipped with three personal computers networked together; one computer is used for operational control of the APT; the second computer is used for data collection and reduction; the third computer is set up to monitor the APT functions. The control room also houses two scanners for collecting data from sensors embedded in the pavement structure. At this time the system used for the data acquisition is the Vishay Measurements Group System 6100. Each scanner unit accepts up to 20 input cards and has the ability to scan at a very high sampling rate, up to 10,000 samples per second per channel.

In this study a gauge array similar to the NCAT study was installed (see Section 3.5). As the APT carriage traversed the pavement, gauges were sampled at a rate of 100 scans per second. However, not all load passes were captured; usually every tenth cycle was recorded. Also no data was collected in between load passes during which the pavement recovered as the APT carriage was lifted in the air and returned to the startup position. This shutdown of the data recording was done in order to save storage space. It 
should be noted that whenever wander was applied, the lateral carriage position was not recorded. Hence, the exact location of the load relative to the embedded gauge array is known only for the initial part of the experiment in which passes were applied without wander.

\subsection{COMPOSITION OF TEST SECTIONS}

The pavement structures (and subgrade) of sections $\mathrm{n} 1$ and $\mathrm{n} 2$ in lanes 1 and 2 respectively (see Figure 3.1.1) are shown in Figure 3.2.1. As can be seen, similar to the N1 and N2 NCAT sections both are comprised of 5 in. $(127 \mathrm{~mm})$ of hot mix asphalt (HMA) overlaying 6 in. (152 mm) of crushed granite aggregate base course, placed on top of an A-4(0) soil serving as subgrade. Materials used to construct the sections were sampled at NCAT and hauled to Indiana for placement in the APT. The properties of each of the pavement components are discussed in more detail in the following subsections. Llenín and Pellinen (2004) and Llenín et al. (2006) provide details on the overall project planning and pavement construction process (see also Appendix C).

\begin{tabular}{|c|c|c|c|}
\hline \multirow{4}{*}{$5 \mathrm{in.}$} & & $\underline{\text { Section } n 1}$ & $\underline{\text { Section } \mathrm{n} 2}$ \\
\hline & \multirow{3}{*}{$\frac{T}{\frac{T}{D}}$} & Mix 1: NMAS 9.5 mm; PG 76-22; AVC 7.5\% & Mix 3: NMAS 9.5 mm; PG 67-22; AVC 7.3\% \\
\hline & & Mix 2: NMAS 19 mm; PG 76-22; AVC 10.2\% & Mix 4: NMAS 19 mm; PG 67-22; AVC 9.7\% \\
\hline & & Mix 2: NMAS 19 mm; PG 76-22; AVC 9.1\% & Mix 4: NMAS $19 \mathrm{~mm} ;$ PG 67-22; AVC 9.4\% \\
\hline $6 \mathrm{in.}$ & 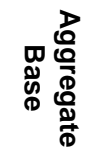 & \multicolumn{2}{|c|}{$\begin{array}{l}\text { Crushed granite material; Average dry unit } \\
\text { weight: } 133.5 \text { pcf }=97 \%\end{array}$} \\
\hline $19 \mathrm{in.}$ & 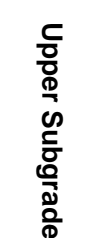 & \multicolumn{2}{|c|}{$\begin{array}{c}\text { A-4(0) soil; Average dry unit weight: } \\
111.5 \text { pcf = Average compaction degree: } \\
93 \%\end{array}$} \\
\hline
\end{tabular}

\section{Lower Subgrade: $90 \%$ compaction}

Figure 3.2.1: Composition of APT test pavements n1 and n2. 
The subgrade in the APT was compacted in lifts, each up to 6 in. (152 mm) thick, using vibratory plate equipment. The average compaction densities for each layer are given in Llenin and Pellinen (2004). Referring to the top 19 in. (483 mm) of the subgrade, the average as-built wet density was 126.9 and 128.0 pcf (2035 and 2052 $\mathrm{kg} / \mathrm{m}^{3}$ ) for sections $\mathrm{n} 1$ and $\mathrm{n} 2$ respectively. The corresponding average moisture contents were $14.7 \%$ and $14.3 \%$. Hence, the following average dry densities were obtained: 110.6 and $112.0 \mathrm{pcf}$ (1773 and $\left.1796 \mathrm{~kg} / \mathrm{m}^{3}\right)$. Since the material was similar to the NCAT subgrade with a maximum laboratory dry density (Proctor modified) of $119.6 \mathrm{pcf}\left(1918 \mathrm{~kg} / \mathrm{m}^{3}\right)$, then the relative compaction degree achieved in the APT was $92.5 \%$ and $93.6 \%$ for sections $\mathrm{n} 1$ and $\mathrm{n} 2$ respectively (an average of $93 \%$ is shown in Figure 3.2.1). The lower portion of the subgrade was compacted to an average wet density of $123.8 \mathrm{pcf}\left(1985 \mathrm{~kg} / \mathrm{m}^{3}\right)$ and water content of $14.7 \%$. The corresponding dry density was therefore $107.9 \mathrm{pcf}\left(1730 \mathrm{~kg} / \mathrm{m}^{3}\right)$ which represents a compaction degree of 90\% (as shown in Figure 3.2.1).

The aggregate base was compacted in a single 6 in. (152.4 mm) lift. The average as-built wet density for this layer was 139.6 and $136.7 \mathrm{pcf}\left(2238\right.$ and $2192 \mathrm{~kg} / \mathrm{m}^{3}$ ) for sections $\mathrm{n} 1$ and $\mathrm{n} 2$ respectively. The corresponding average moisture contents were 3.5\% and 3.6\%. Hence, the following average dry densities were obtained: 134.9 and $131.9 \mathrm{pcf}$ (2163 and $2115 \mathrm{~kg} / \mathrm{m}^{3}$ ). Since the material was similar to the NCAT base, having a maximum laboratory dry density (Proctor modified) of 137.9 pcf (2211 $\mathrm{kg} / \mathrm{m}^{3}$ ), then the relative compaction degree achieved in the APT was $97.8 \%$ and $95.6 \%$ for sections $\mathrm{n} 1$ and $\mathrm{n} 2$ respectively (an average of $97 \%$ is shown in Figure 3.2.1).

The HMA mixes in the APT had similar composition to the corresponding NCAT mixes. The mixes were produced by a local contractor in Indiana using NCAT aggregates. The HMA course in each lane was made of two mixes and constructed in three lifts. With reference to Figure 3.2.1, the surface lifts (mixes 1 and 3), were $1.0 \mathrm{in}$. $(25.4 \mathrm{~mm}$ ) thick while the intermediate and bottom lifts (mixes 2 and 4), were $4.0 \mathrm{in}$. $(101.6 \mathrm{~mm})$ thick constructed in two 2 in. $(50.8 \mathrm{~mm})$ lifts. The average as-constructed air void content for the three lifts in Section $\mathrm{n} 1$ was as follows (top to bottom): $7.5 \%$, $10.2 \%$ and $9.1 \%$. The average as-constructed air void content for the three lifts in 
Section n2 was: 7.3\%, 9.7\% and 9.4\%. Recall from Chapter 2 (Subsection 2.2.4) that at NCAT the corresponding average void contents were about 6 to $7 \%$ for all lifts.

\subsection{MECHANICAL TESTING AND PRELIMINARY ANALYSIS}

\subsubsection{Resilient Modulus of Unbound Materials}

The accepted mathematical expression for representing resilient modulus test results, unlike equation 2.3.1, is (Uzan, 1985; 1992; Witczak and Uzan, 1988):

$$
M_{R}=\left(k_{1} \cdot P_{a}\right) \cdot\left(\frac{\theta}{P_{a}}\right)^{k_{2}} \cdot\left(\frac{\tau_{o c t}}{P_{a}}+1\right)^{k_{3}}
$$

where $P_{a}$ is atmospheric pressure, $\theta=\sigma_{1}+\sigma_{2}+\sigma_{3}$ is the bulk stress, $\tau_{\text {oct }}$ is the octahedral shear stress defined by the expression $9 \cdot \tau_{\text {oct }}^{2}=\left(\sigma_{1}-\sigma_{2}\right)^{2}+\left(\sigma_{1}-\sigma_{3}\right)^{2}+\left(\sigma_{2}-\sigma_{3}\right)^{2}$. Peak applied (total) principal stresses in the triaxial apparatus are dented as $\sigma_{1}, \sigma_{2}$ and $\sigma_{3}$; these are related to the AASHTO T307 terminology as follows: $\sigma_{1}=S_{3}+S_{c}+S_{s}$ and $\sigma_{2}=\sigma_{3}=S_{3}$ in which $S_{3}$ in the confining pressure, $S_{c}$ is the peak cyclic stress and $S_{s}$ is the uniaxial static or seating load. $S_{s}$ is relatively small, with values of about $0.1 \cdot S_{c}$. The three model parameters: $k_{1}, k_{2}$ and $k_{3}$ (unitless) represent the specific material within the range of applied stresses and at given moisture $(\omega)$ and density levels.

In typical pavement applications, compacted unbound materials are unsaturated with the moisture phase in tension (i.e., negative pore pressures). In this case it was found advantageous to modify equation 3.3.1 such that it includes the influence of suction on the modulus. Assuming that increased suction has similar influence as increased confining stresses, the resulting modified equation is (see also Lytton et al., 1993; Andrei et al., 2004):

$$
M_{R}=\left(k_{1} \cdot P_{a}\right) \cdot\left(\frac{\theta+k_{6}}{P_{a}}\right)^{k_{2}} \cdot\left(\frac{\tau_{o c t}}{P_{a}}+1\right)^{k_{3}}
$$


in which the additional (positive) parameter $k_{6}$, having units of stress, represents suction effects. If we further assume that all moisture sensitivity is lumped into $k_{6}$, i.e., $k_{6}=k_{6}(\omega)$, then the remaining three parameters in equation 3.3.2 (namely: $k_{1}, k_{2}$ and $k_{3}$ ) are independent of moisture content (all four parameters remain density dependent). The parameter $k_{6}$ should attain a value of zero whenever there is no moisture present in the material and also when the moisture levels are high and the suction has negligible effect on the modulus. Between these two extremes $k_{6}$ will arrive at some maximum (positive) value.

Equation 3.3.2 was applied to analyze the resilient modulus test data for the subgrade and base materials presented in Chapter 2. For this purpose, the numerical values of the parameters were manipulated by a nonlinear optimization algorithm until a best fit was achieved between the model projections and the test results. The goodness of fit was defined based on absolute relative errors. Three data sets were analyzed, namely: subgrade soil compacted to $96 \%$ (see Table 2.3.1), aggregate base compacted to $93 \%$ (Table 2.3.2) and aggregate base compacted to $97.5 \%$. In the latter case, a synthetic set of test data was generated using equation 2.3.1 with three levels of confining pressure (5, 10 and 15 psi or equivalently 34.5, 68.9 and $103.4 \mathrm{kPa}$ ) and five levels of cyclic stress (5, 10, 15, 20 and 25 psi or equivalently 34.5, 68.9, 103.4, 137.9 and $172.4 \mathrm{kPa}$ ).

The resulting values of the equation 3.3.2 parameters, in each of the three cases, are summarized in Table 3.3.1 (with $P_{a}=14.5 \mathrm{psi}$ ). As can be seen, $k_{1}$ is positive and equals about 500 in all three cases; $k_{2}$ is also positive and ranges between 0.8 and 1.0; $k_{3}$ is negative and equals about -0.75 for the high density cases (i.e., $96 \%$ subgrade and $97.5 \%$ base). For the low density base $k_{3}$ has doubled in value. The suction component $k_{6}$ was found to equal about $5 \mathrm{psi}(34.5 \mathrm{kPa}$ ) for the subgrade soil at $7.2 \%$ moisture; at moisture levels higher than $9.7 \%$ it was found negligible. For the base material at about $5.4 \%$ water content $k_{6}$ is seen to increase in value with density, from 2 psi at $93 \%$ 
compaction to 7.2 psi at $97.5 \%$ compaction; at a moisture level of $9.8 \% k_{6}$ was found negligible (in the low density case).

The as-constructed subgrade moisture content at NCAT was about $10.5 \%$ (see Chapter 2, Subsection 2.2.2) and about 14.5\% in the APT (see Section 3.3). Based on the above results the suction component in the subgrade should be negligible. The asconstructed base moisture content was 6.5\% at NCAT (see Chapter 2, Subsection 2.2.3) and about 3.5\% in the APT (see Section 3.3). Hence, the suction component in the base can be estimated at around 7 psi (48.3 $\mathrm{kPa})$; this is equivalent to a weight of $86 \mathrm{in}$. (2.2 m) of base material with a total (wet) density of $140 \mathrm{pcf}\left(2245 \mathrm{~kg} / \mathrm{m}^{3}\right)$.

Table 3.3.1: Resilient modulus of unbound materials (calibrated equation 3.3.2 parameters).

\begin{tabular}{|c|c|c|c|c|c|}
\hline Material & Compaction, $\%$ & $\boldsymbol{k}_{\mathbf{1}}$ & $\boldsymbol{k}_{\mathbf{2}}$ & $\boldsymbol{k}_{\mathbf{3}}$ & $\boldsymbol{k}_{\mathbf{6}}, \mathbf{p s i}$ \\
\hline \multirow{2}{*}{ Subgrade Soil } & 96.0 & 554 & 0.827 & -0.770 & $\begin{array}{c}4.8(\omega=7.2 \%) \\
0.0(\omega=9.7 \%) \\
0.0(\omega=20.1 \%)\end{array}$ \\
\hline \multirow{2}{*}{ Aggregate Base } & 93.0 & 477 & 0.999 & -1.580 & $\begin{array}{l}2.0(\omega=5.3 \%) \\
0.0(\omega=9.8 \%)\end{array}$ \\
\cline { 2 - 6 } & 97.5 & 527 & 0.833 & -0.715 & $7.2(\omega=5.5 \%)$ \\
\hline
\end{tabular}

The entire set of test data and model forecasts are cross plotted in Figure 3.3.1 (log-log scale). The goodness of fit may be graphically assessed from this figure. As can be seen all the data points fall very close to the equality line (oblique dashed line). It may also be seen that the resilient modulus of the subgrade at $96 \%$ compaction (square markers) ranges between 4,500 and 15,000 psi (31 and $103 \mathrm{MPa}$ ). The aggregate base at 93\% (triangular markers) has modulus values in the range of 4,000 to 10,000 psi (28 to $69 \mathrm{MPa}$ ). The resilient modulus of the $97.5 \%$ base (circular markers) has a range of 13,000 to 21,000 psi (90 to $145 \mathrm{MPa}$ ). It should be noted that, for the tested conditions, considerable overlap is seen in the modulus ranges of the subgrade and base. This means that they may exhibit comparable stiffness as part of the pavement system. 


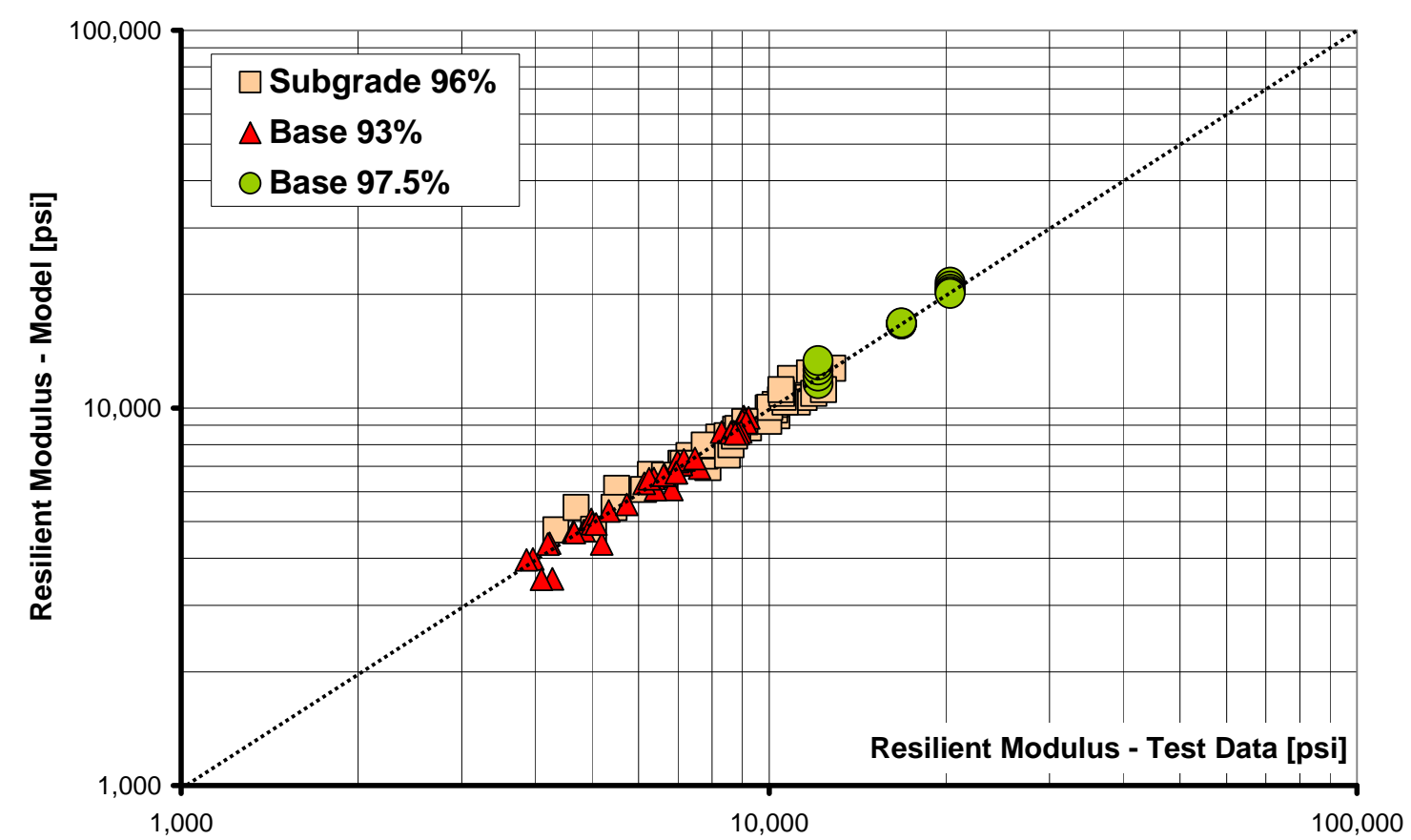

Figure 3.3.1: Resilient modulus of unbound materials - a cross plot of calibrated equation 3.3.2 values and test data.

\subsubsection{HMA Complex Modulus}

In this subsection the complex modulus test results presented in Chapter 2 (Tables 2.3.3 to 2.3.6) are analyzed. In general terms the interpretation involves horizontal shifting, along the frequency axis, of the measured dynamic modulus and phase angle data obtained at different temperatures. This is done with respect to a pre-selected reference temperature until two separate but continuous curves are attained. The first is the socalled 'dynamic modulus master curve' and the second 'phase angle master curve'. The analysis performed herein follows the approach recommended in Levenberg and Shah (2008). This method is slightly different than the common/usual methods because use is made of both dynamic modulus and phase angle data, simultaneously, to obtain the master curves. This approach was chosen herein because it was developed specifically for asphalt mixtures. A short theoretical background and description of the approach is provided hereafter.

When a time varying uniaxial stress $\sigma(t)$ is applied to a linear viscoelastic solid at a given test temperature $T_{0}$, in the form: $\sigma(t)=\sigma_{0} \cdot \exp (i \cdot \omega \cdot t)$ with $i^{2}=-1, \sigma_{0}$ as 
the stress amplitude (constant) and $\omega$ as the angular frequency (units of radians per second), the resulting steady state strain response is also sinusoidal. The quotient of stress and strain in the frequency domain is may be represented by a complex number:

$$
E^{*}=\left|E^{*}\right| \cdot(\cos \phi+i \cdot \sin \phi)=E_{1}+i \cdot E_{2}
$$

in which $E^{*}$ is the material's complex modulus, $\left|E^{*}\right|$ is the dynamic modulus and $\phi$ denotes the phase lag by which the strain lags behind the applied stress. These quantities, although not shown explicitly, are functions of both $\omega$ and $T_{0}$.

The components of the complex modulus, $E_{1}$ and $E_{2}$ in equation 3.3.3, can be expressed using one fundamental viscoelastic function known as the relaxation spectrum $h$ and an additional material constant known as the equilibrium modulus $E_{\infty}$ :

$$
\begin{aligned}
& E_{1}\left(\omega, T_{0}\right)=E_{\infty}+\int_{0}^{\infty} h\left(\tau, T_{0}\right) \cdot \frac{\omega^{2} \cdot \tau^{2}}{1+\omega^{2} \cdot \tau^{2}} \cdot d(\ln \tau) \\
& E_{2}\left(\omega, T_{0}\right)=\int_{0}^{\infty} h\left(\tau, T_{0}\right) \cdot \frac{\omega \cdot \tau}{1+\omega^{2} \cdot \tau^{2}} \cdot d(\ln \tau)
\end{aligned}
$$

As can be seen, the relaxation spectrum has units of stress and is a function of time $\tau$ and temperature $T_{0}$, i.e., $h=h\left(\tau, T_{0}\right)$. The equilibrium modulus $E_{\infty}$ is temperature independent, defined as: $E_{\infty}=\lim _{\omega \rightarrow 0} E_{1}(\omega)=\lim _{t \rightarrow \infty} E(t)$ in which $E(t)$ is the viscoelastic relaxation modulus (units of stress).

Equations 3.3.4 and 3.3.5 are appropriate for a given constant test or reference temperature, $T_{0}$. The assumption of thermo-rheological simplicity (Schwarzl and Staverman, 1952) states that these relations can remain applicable for a different (constant) temperature, $T$, simply by replacing physical time, $\tau$, with reduced (or pseudo) time, $\tau_{r}$, defined as: $\tau_{r}=\tau / a_{T}$, where $a_{T}=a_{T}\left(T, T_{0}\right)$ is the so-called timetemperature shift factor which is a unitless function of temperature only. Due to the reciprocal nature of time and frequency, the reduced angular frequency, $\omega_{r}$, is simply obtained by $\omega_{r}=\omega \cdot a_{T}$ with $\omega$ as the applied angular frequency. For a certain class of 
polymers (Plazek, 1996), and for a limited range of temperatures, $a_{T}$ tend to follow the Williams-Landel-Ferry equation (Williams et al., 1955):

$$
\log \left(a_{T}\right)=\frac{-c_{1} \cdot\left(T-T_{0}\right)}{c_{2}+\left(T-T_{0}\right)}
$$

where $c_{1}$ and $c_{2}$ are both positive constants $\left(c_{1}\right.$ is unitless and $c_{2}$ has units of temperature). This equation was found applicable to HMA mixtures (e.g., Di Benedetto et al., 2007).

As suggested in Levenberg and Shah (2008), a mathematical expression for the relaxation spectrum $h\left(\tau, T_{0}\right)$ of the following form is assumed:

$$
h(\tau)=a_{1} \cdot \exp \left(-a_{2} \cdot\left[\ln (\tau)-\ln \left(a_{3}\right)\right]^{2}\right)
$$

where $a_{1}, a_{2}$ and $a_{3}$ are all temperature dependent positive constants. The variable $a_{1}$ has units of modulus, $a_{2}$ is unitless, and $a_{3}$ has units of time (similar to $\tau$ ). This equation, along with equations 3.3.4, 3.3.5 and 3.3.6 were used to fit the complex modulus test data given in Chapter 2 by Tables 2.3.5 to 2.3.8 (separate analysis for each case). In this process six parameters needed evaluation, namely: $a_{1}, a_{2}, a_{3}$ (equation 3.3.7), $E_{\infty}$ (equation 3.3.4), $c_{1}$ and $c_{2}$ (equation 3.3.6). Their numerical value obtained simultaneously using a nonlinear minimization algorithm where the goodness of fit was defined based on relative errors. The chosen reference temperature was $15.5^{\circ} \mathrm{C}$. A summary of their derived values, for each of the four mix types, is given in Table 3.3.2.

The test data and corresponding master curves are shown in Figures 3.3.2 to 3.3.5. In these figures the dynamic modulus is depicted on the left ordinate and the phase angle on the right ordinate. The abscissa represents reduced frequency $f_{r}$ defined by the expression: $\omega_{r}=2 \cdot \pi \cdot f_{r}$. In Figures 3.3.6 and 3.3.7 the master curves are superimposed for graphical comparison. The four dynamic modulus muster curves are all plotted in Figure 3.3.6, and the four phase angle master curves are plotted in Figure 3.3.7. It may be seen that mixes 1 and 3, and separately mixes 2 and 4, have very similar master curves for all practical purposes. 
Table 3.3.2: Complex modulus analysis results for a reference temperature of $15.5^{\circ} \mathrm{C}$ based on the approach in Levenberg and Shah (2008).

\begin{tabular}{|c|c|c|c|c|c|c|}
\hline \multirow{2}{*}{ Mix } & \multicolumn{2}{|c|}{$\begin{array}{c}\text { Relaxation Modulus Parameters } \\
\text { (equation 3.3.7) }\end{array}$} & $\begin{array}{c}\text { Equilibrium } \\
\text { Modulus }\end{array}$ & \multicolumn{2}{c|}{$\begin{array}{c}\text { Time-Temperature } \\
\text { Shifting Parameters } \\
\text { (equation 3.3.6) }\end{array}$} \\
\cline { 2 - 7 } & $\begin{array}{c}a_{1}, \mathrm{MPa} \\
(\mathrm{ksi})\end{array}$ & $a_{2} \cdot 10^{3}$ & $a_{3} \cdot 10^{5}, \mathrm{~s}$ & $\begin{array}{c}E_{\infty}, \mathrm{MPa} \\
(\mathrm{ksi})\end{array}$ & $c_{1}$ & $c_{2},{ }^{\circ} \mathrm{C}\left({ }^{\circ} \mathrm{F}\right)$ \\
\hline 1 & $\begin{array}{c}1,983 \\
(287.6)\end{array}$ & 9.84 & 3.91 & $\begin{array}{c}172 \\
(24.9)\end{array}$ & 26.8 & $\begin{array}{c}215.7 \\
(420.3)\end{array}$ \\
\hline 2 & $\begin{array}{c}2,421 \\
(351.1)\end{array}$ & 10.63 & 8.81 & $\begin{array}{c}159 \\
(23.1)\end{array}$ & 35.6 & $\begin{array}{c}338.2 \\
(640.8)\end{array}$ \\
\hline 3 & $\begin{array}{c}1,822 \\
(264.3)\end{array}$ & 12.10 & 17.79 & $\begin{array}{c}91 \\
(13.2)\end{array}$ & 44.1 & $\begin{array}{c}376.4 \\
(709.5)\end{array}$ \\
\hline 4 & $\begin{array}{c}2,223 \\
(322.4)\end{array}$ & 11.55 & 16.38 & $\begin{array}{c}272 \\
(39.5)\end{array}$ & 34.5 & $\begin{array}{c}311.9 \\
(593.4)\end{array}$ \\
\hline
\end{tabular}

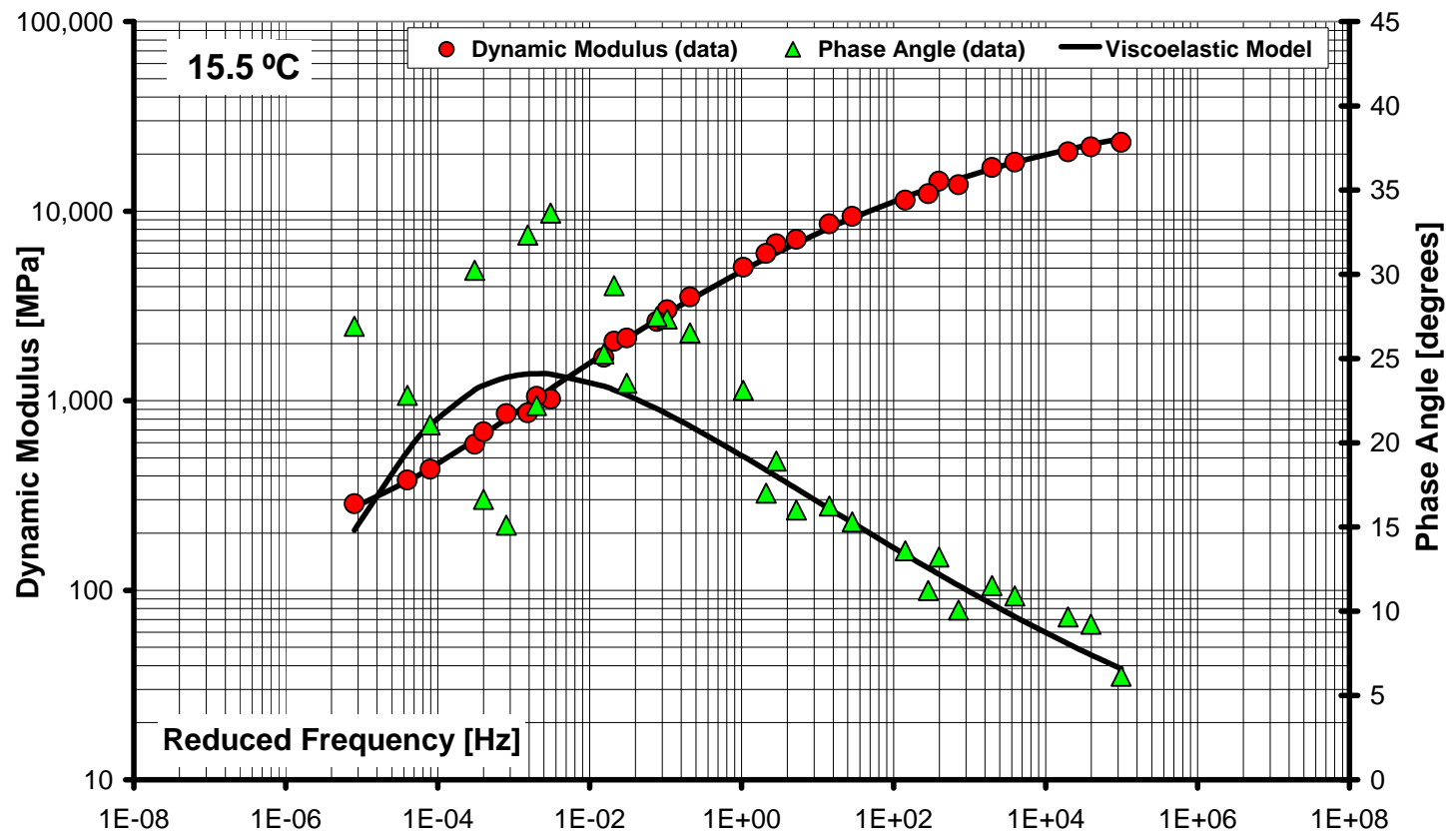

Figure 3.3.2: Mix 1 dynamic modulus and phase angle master curves @ $15.5^{\circ} \mathrm{C}$. 


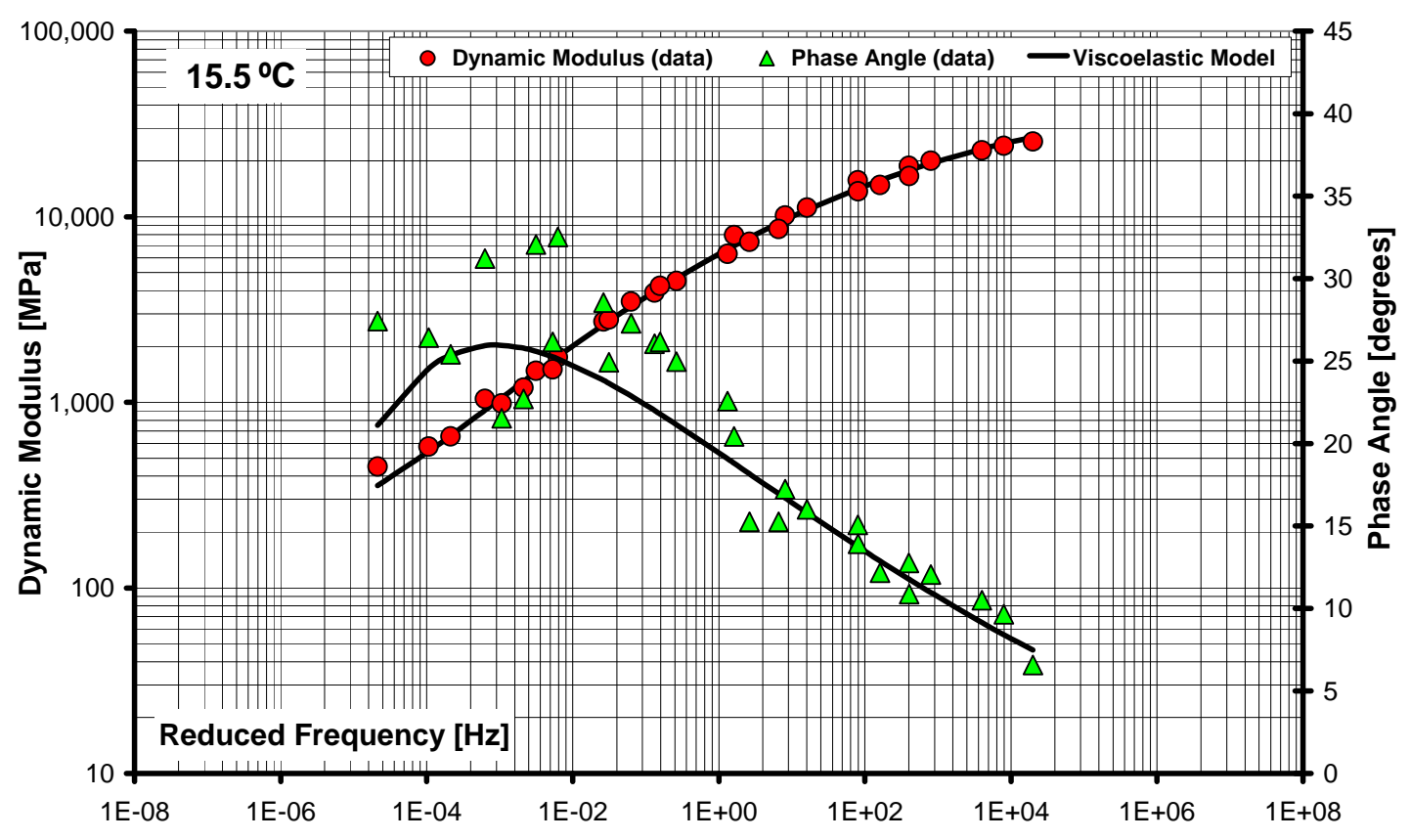

Figure 3.3.3: Mix 2 dynamic modulus and phase angle master curves @ $15.5^{\circ} \mathrm{C}$.

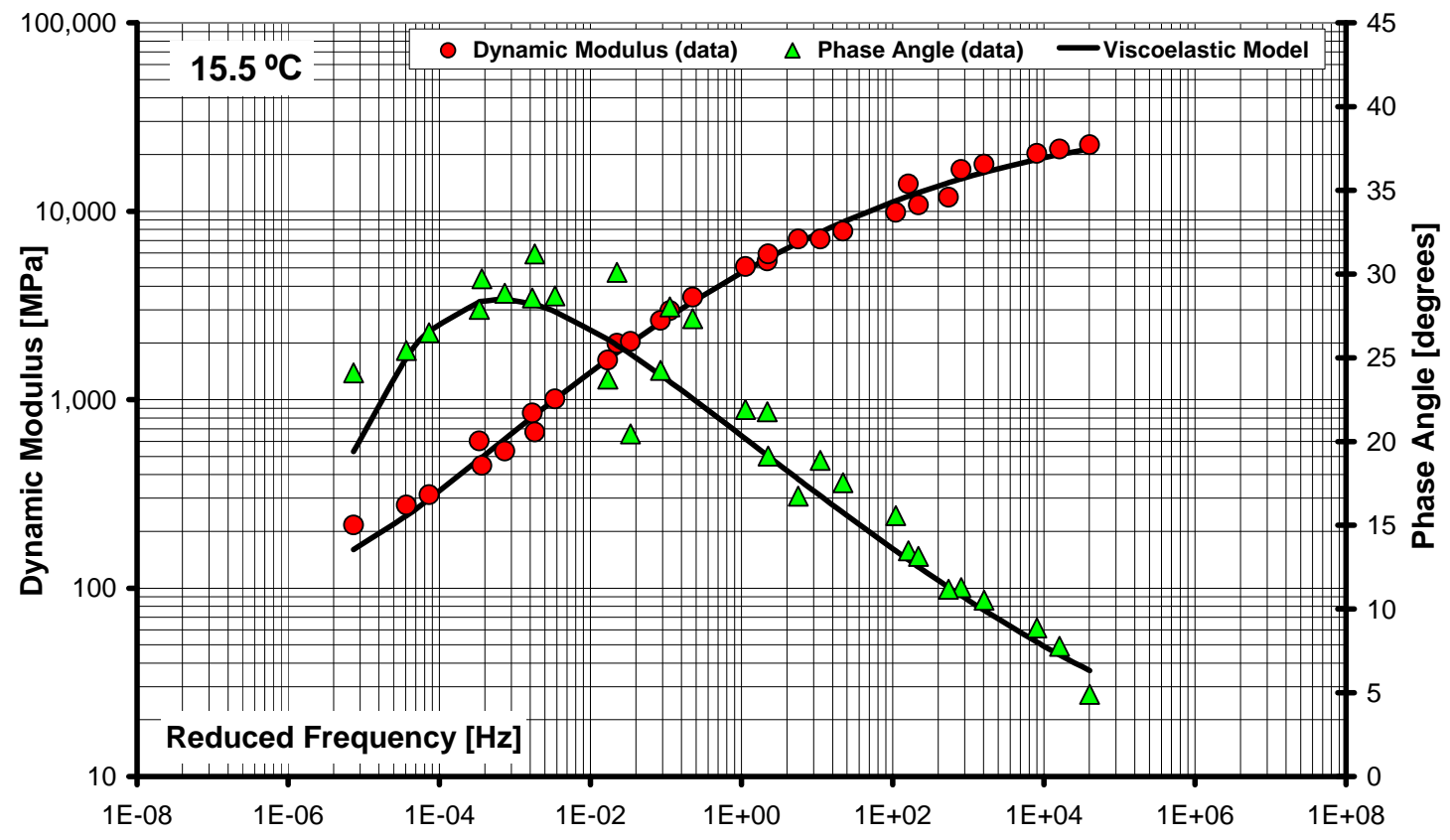

Figure 3.3.4: Mix 3 dynamic modulus and phase angle master curves @ $15.5^{\circ} \mathrm{C}$. 


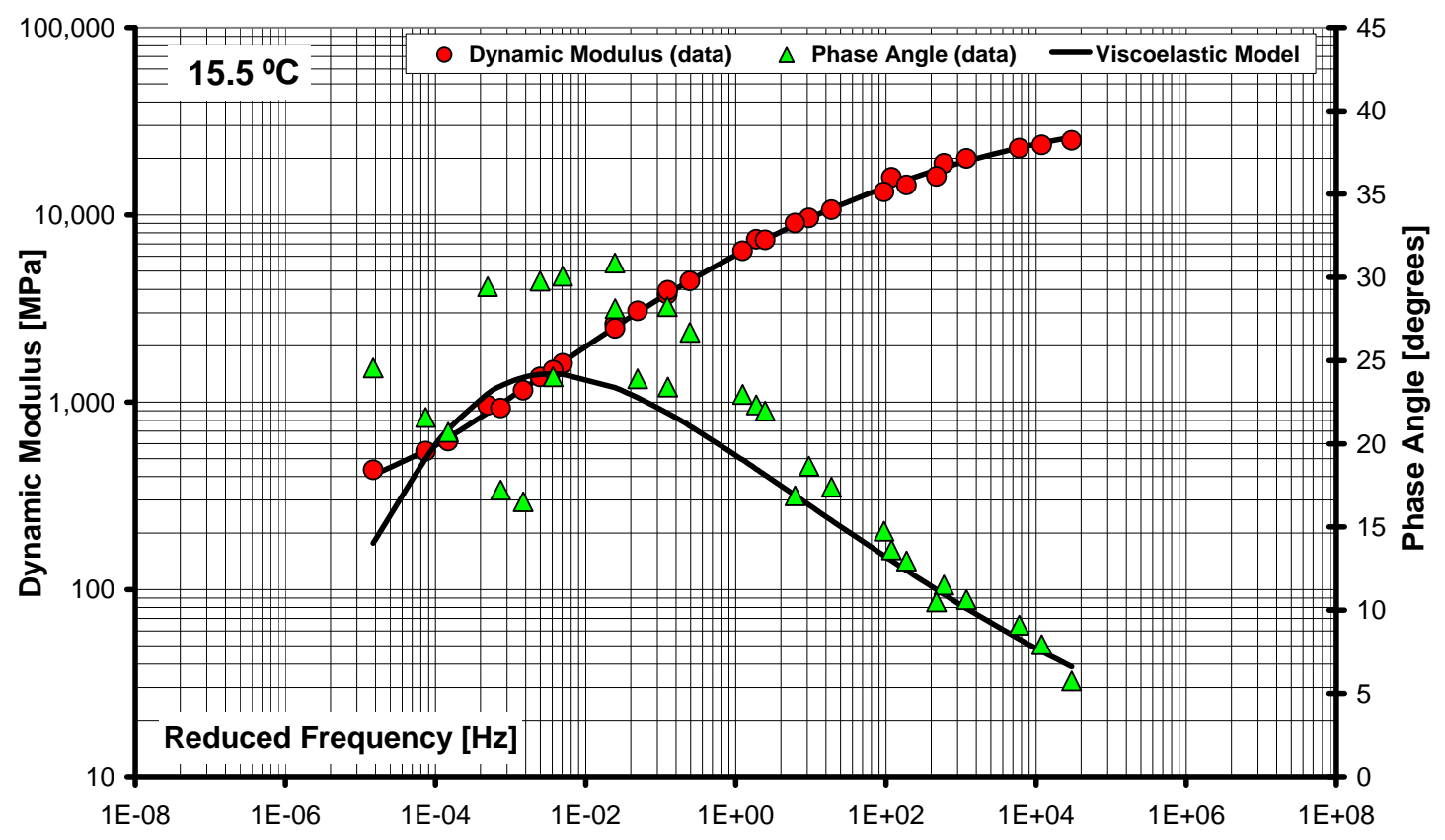

Figure 3.3.5: Mix 4 dynamic modulus and phase angle master curves @ $15.5^{\circ} \mathrm{C}$.

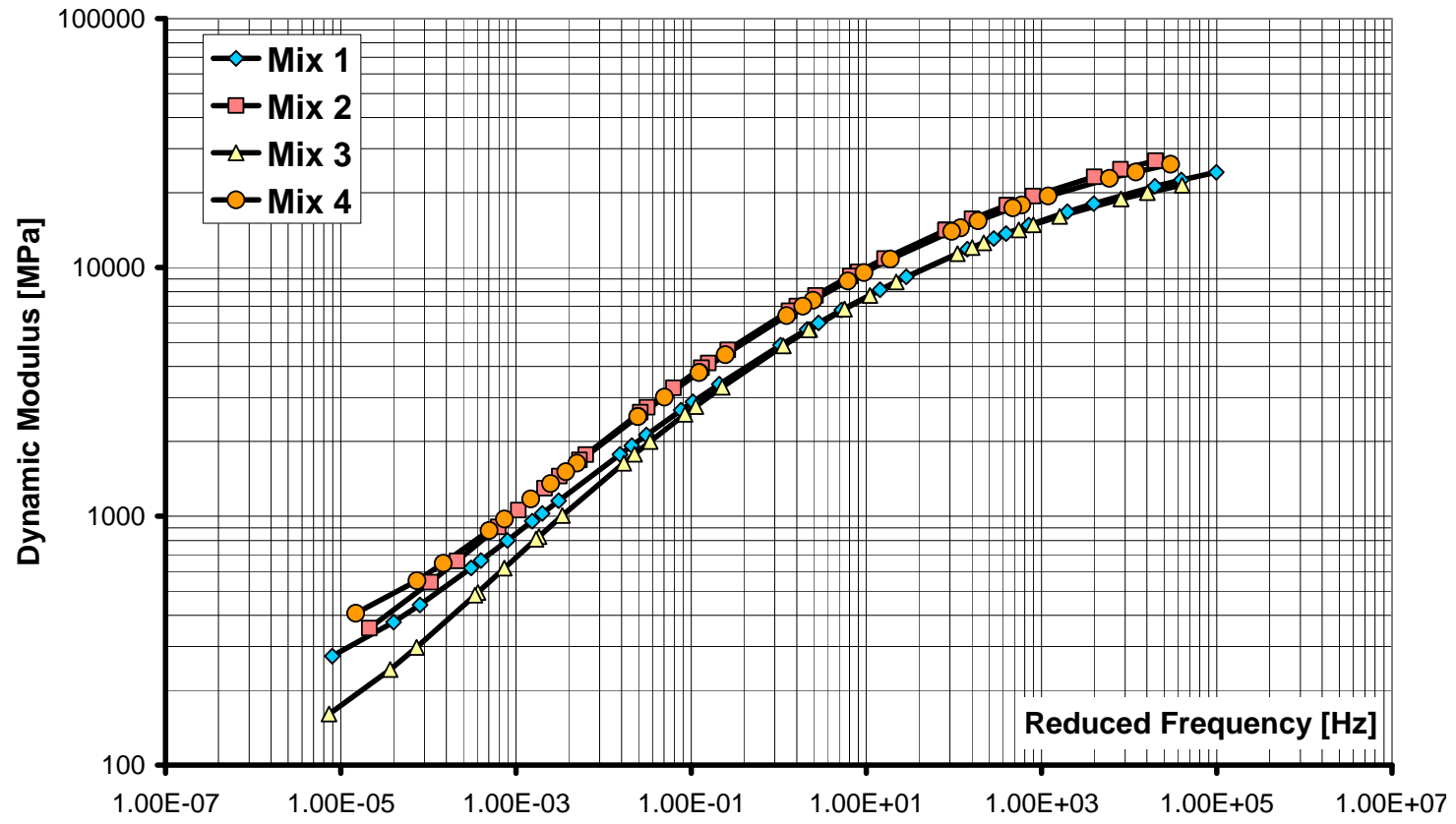

Figure 3.3.6: Superimposed dynamic modulus master curves @ $15.5^{\circ} \mathrm{C}$ for mixes 1 to 4 . 


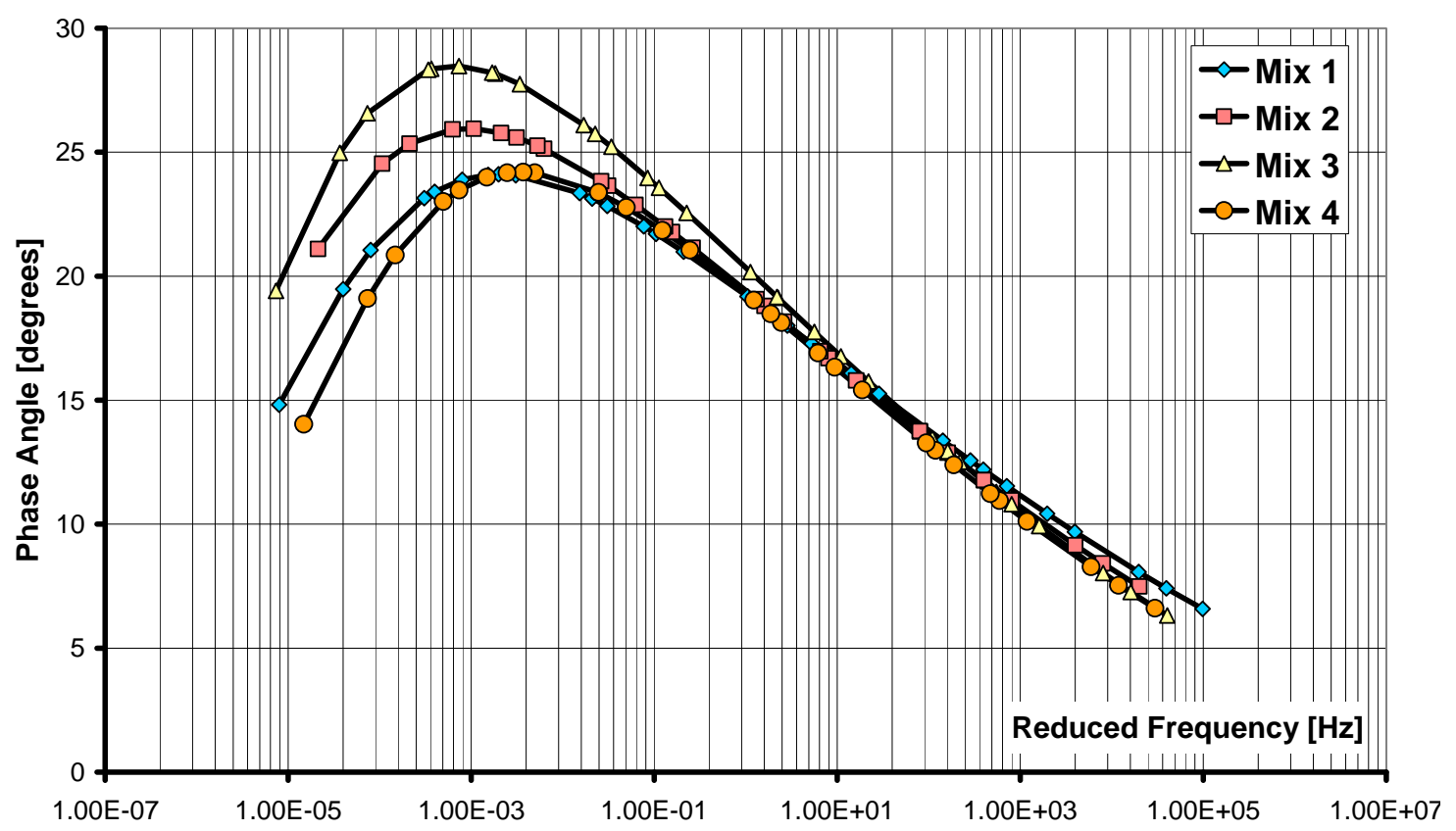

Figure 3.3.7: Superimposed phase angle master curves @ $15.5^{\circ} \mathrm{C}$ for mixes 1 to 4 .

\subsubsection{Falling Weight Deflections}

FWD testing was conducted in the APT on June 14, 2004, before passes were applied (embedded instrumentation was not activated during the test). The FWD loading plate was $11.81 \mathrm{in.} \mathrm{(300} \mathrm{mm)} \mathrm{in} \mathrm{diameter.} \mathrm{A} \mathrm{set} \mathrm{of} \mathrm{nine} \mathrm{geophones} \mathrm{was} \mathrm{used,} \mathrm{located} \mathrm{at} \mathrm{the}$ following offset distances from the center of the plate: $0,8,12,18,24,36,48,60$ and 72 in. (0, 0.20, 0.30, 0.46, 0.61, 0.91, 1.22, 1.52 and $1.83 \mathrm{~m})$. Testing was performed in centers of lane 1 and lane 2. Six drop sequences were applied in each lane, with each drop consisting of three load levels: 65, 85 and 105 psi (0.448, 0.586 and $0.724 \mathrm{MPa}$ ). The pavement surface temperature during the test was $86.5^{\circ} \mathrm{F}\left(30.3^{\circ} \mathrm{C}\right)$. The average peak deflections measured at each load level are shown in Table 3.3.3. These results are plotted in Figure 3.3.8 in which solid lines represent $\mathrm{n} 1$ deflections and dashed lines represent n2 deflections; the three different marker types represent the three load levels. As can be seen, when comparing the response between the $\mathrm{n} 1$ and $\mathrm{n} 2$ sections, the deflection basins are very similar for offset distances greater than $0.46 \mathrm{~m}$ (18 in.). Closer to the loading plate, the deflections in lane 2 are slightly but consistently larger. 
Table 3.3.3: Peak FWD deflections measured in the center of sections $\mathrm{n} 1$ and $\mathrm{n} 2$.

\begin{tabular}{|c|c|c|c|c|c|c|c|c|}
\hline \multirow{2}{*}{ Lane } & $\begin{array}{c}\text { Load Level } \\
\text { MPa, }(\mathbf{p s i})\end{array}$ & $\begin{array}{c}\text { D0 } \\
{[\boldsymbol{\mu m}]}\end{array}$ & $\begin{array}{c}\text { D1 } \\
{[\boldsymbol{\mu m}]}\end{array}$ & $\begin{array}{c}\text { D2 } \\
{[\boldsymbol{\mu} \mathbf{m}]}\end{array}$ & $\begin{array}{c}\mathbf{D 3} \\
{[\boldsymbol{\mu m}]}\end{array}$ & $\begin{array}{c}\mathbf{D 4} \\
{[\boldsymbol{\mu m}]}\end{array}$ & $\begin{array}{c}\mathbf{D 5} \\
{[\boldsymbol{\mu m}]}\end{array}$ & $\begin{array}{c}\mathbf{D 6} \\
{[\boldsymbol{\mu} \mathbf{m}]}\end{array}$ \\
\hline \multirow{4}{*}{$\mathrm{n} 1$} & $0.448(65)$ & 501.9 & 360.0 & 268.0 & 106.8 & 47.7 & 28.1 & 18.8 \\
\cline { 2 - 9 } & $0.586(85)$ & 661.3 & 482.6 & 362.7 & 149.2 & 67.6 & 39.0 & 26.4 \\
\cline { 2 - 9 } & $0.724(105)$ & 826.3 & 610.5 & 459.4 & 194.9 & 88.4 & 51.4 & 34.6 \\
\hline \multirow{4}{*}{$\mathrm{n} 2$} & $0.448(65)$ & 576.4 & 413.7 & 298.7 & 117.3 & 50.6 & 29.2 & 19.5 \\
\cline { 2 - 9 } & $0.586(85)$ & 750.1 & 547.1 & 395.1 & 158.2 & 70.3 & 39.6 & 26.3 \\
\cline { 2 - 9 } & $0.724(105)$ & 941.4 & 697.2 & 501.3 & 202.4 & 91.5 & 51.5 & 34.0 \\
\hline
\end{tabular}

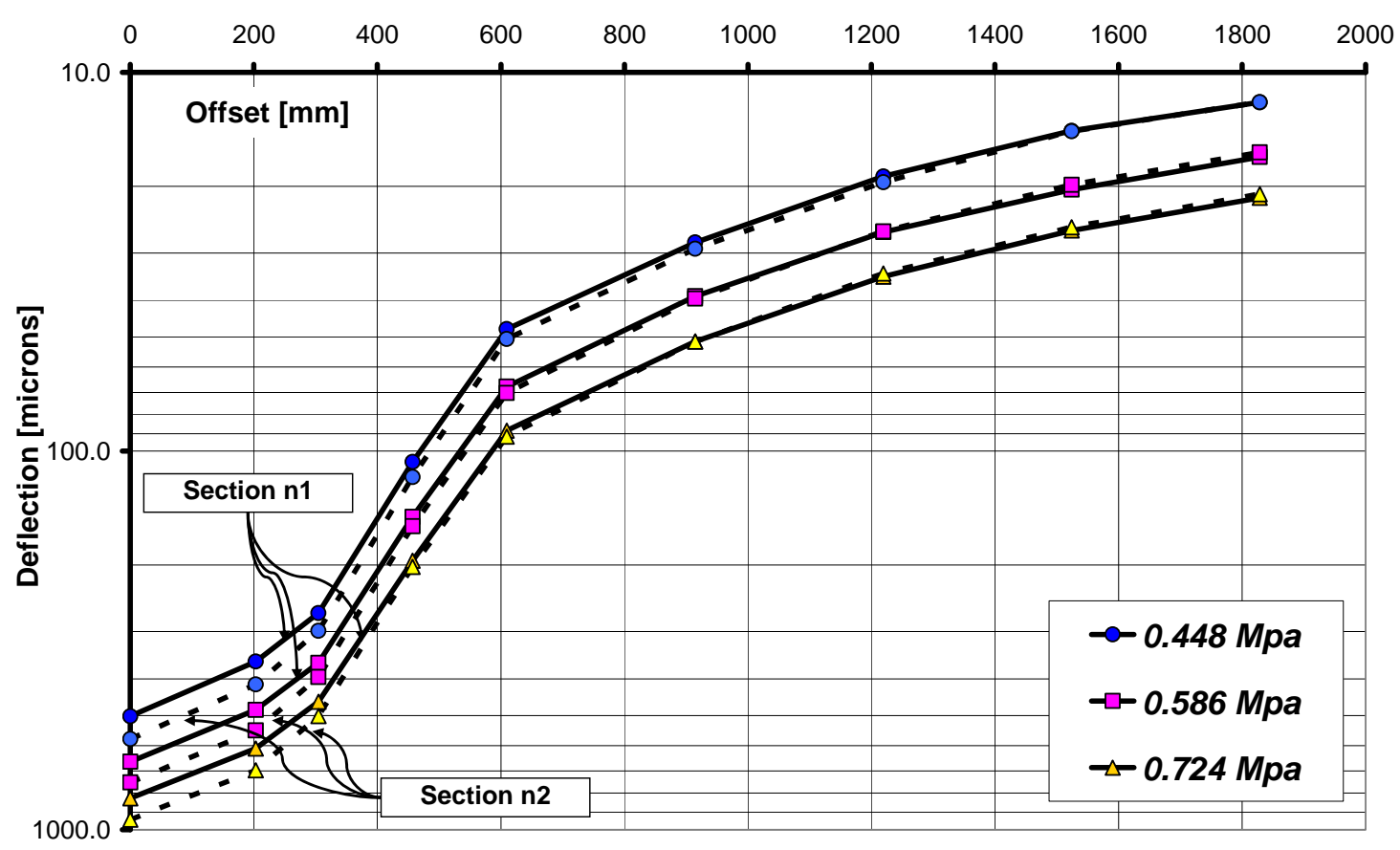

Figure 3.3.8: Peak FWD deflections measured in the center of sections n1 and n2.

\subsection{LOADING HISTORY AND ANALYSIS DATASET}

\subsubsection{Application of Load Passes}

Passes in the APT were applied via a dual wheel assembly loaded to 15,000 lb (6810 $\mathrm{kg})$. Each wheel was equipped with a Goodyear radial Unisteel tire model G159A (designation 11R22.5) inflated to 100 psi (0.70 MPa). All passes were applied in unidirectional mode, some without wander but most with wander (indicated by ' $w$ ' in the following tables). 
Loading of Section n1 began on July 19, 2004. About 90,000 passes were applied by August 11, 2004, all without wander. The last 2,500 cycles where applied overnight, during which a bond failure occurred between the surface and intermediate HMA lifts. At the onset of failure, the surface lift in the wheel path area was sheared-off in the direction of loading, exposing the intermediate HMA lift. Subsequently the loading of lane 1 was stopped, and the APT loading frame was switched to lane 2. Section $\mathrm{n} 2$ incurred about 2,500 passes beginning September 13, 2004, before it became apparent that another bond failure was rapidly developing, again at the interface between the surface and intermediate HMA lifts. Subsequently, it was decided not to wait for complete shear failure, but to mill and repave the surface HMA lift in both sections. This sequence of events is summarized in Table 3.4.1.

Table 3.4.1: APT pass application log for sections $\mathrm{n} 1$ and $\mathrm{n} 2$ (original structure).

\begin{tabular}{|c|c|c|c|}
\hline Date & Section & Cumulative Passes & Days \\
\hline 19-Jul-04 & \multirow{11}{*}{$\mathrm{n} 1$} & 0 & 0 \\
\hline 22-Jul-04 & & 5,000 & 3 \\
\hline 23-Jul-04 & & 10,000 & 4 \\
\hline 25-Jul-04 & & 20,000 & 6 \\
\hline 27-Jul-04 & & 30,000 & 8 \\
\hline 29-Jul-04 & & 40,000 & 10 \\
\hline 30-Jul-04 & & 50,000 & 11 \\
\hline 2-Aug-04 & & 60,000 & 14 \\
\hline 6-Aug-04 & & 70,000 & 18 \\
\hline 9-Aug-04 & & 80,000 & 21 \\
\hline 11-Aug-04 & & 90,000 & 23 \\
\hline \multicolumn{4}{|c|}{$\begin{array}{l}\text { Loading stopped due to bond failure between top and intermediate } \\
\text { HMA layers. }\end{array}$} \\
\hline 13-Sep-04 & $\mathrm{n} 2$ & 2,500 & 1 \\
\hline
\end{tabular}

Resurfacing of the surface HMA lift took place between September 13 and September 28, 2004 (no construction details are available). Reloading of section n2 began on the latter date and continued until February 1, 2005, at which point 187,500 passes had been applied. The first 40,000 passes were applied without wander; wheel 
wander was employed for the reminder of the test. Trafficking of section n2 was discontinued because another bond failure was seen to take place, this time at the interface between the intermediate and bottom lifts. This sequence of events is shown in Table 3.4.2.

Table 3.4.2: APT pass application log for Section $\mathrm{n} 2$ (rehabilitated structure).

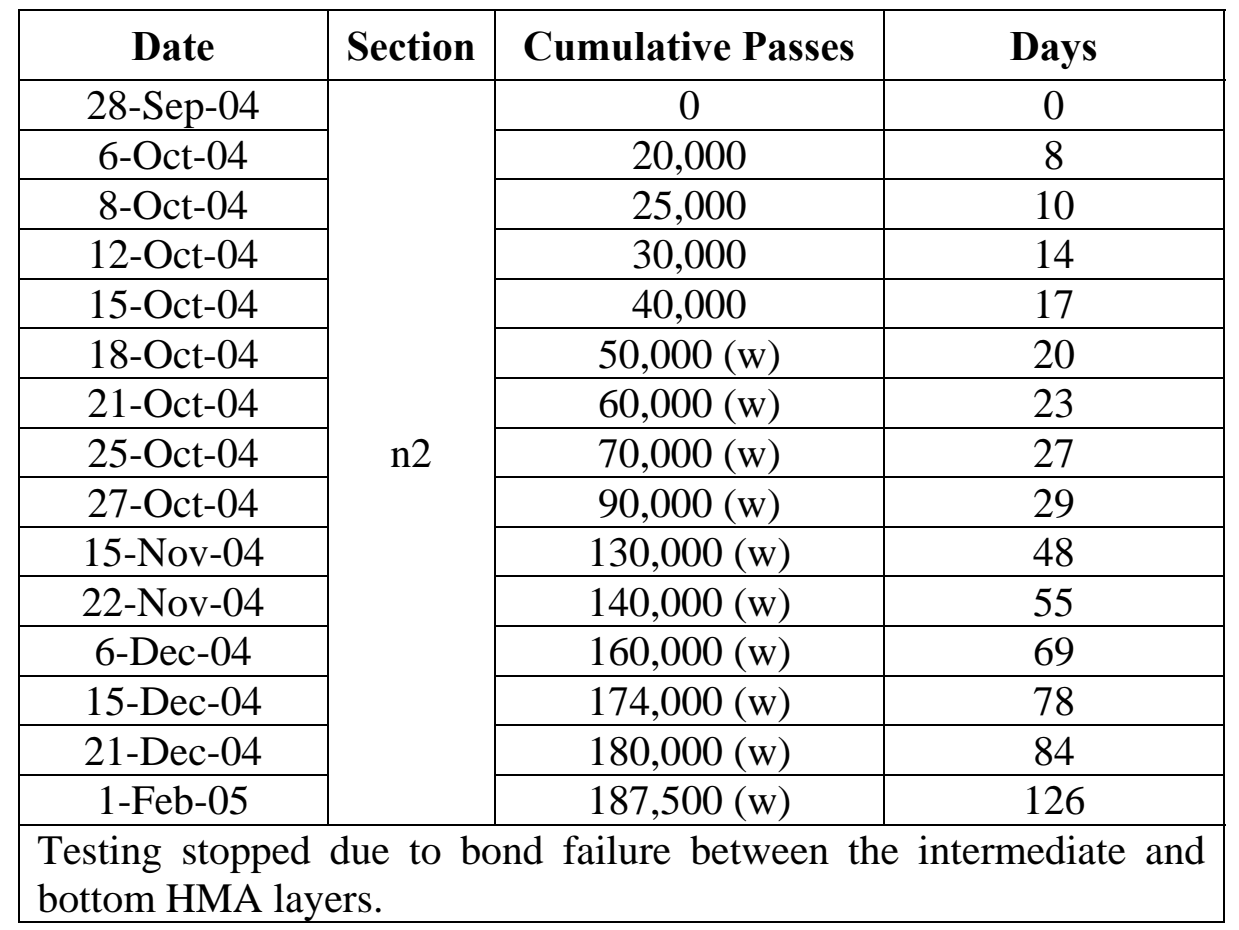

Water was introduced to the subgrade pit beginning June 27, 2005 (details can be found in Appendix C). This was accomplished by localized removal of the HMA in lane 2 (by means of saw cutting and coring), and inundating the openings. On July 14, 2005, a dynamic cone penetration test indicated that the shear strength of the subgrade had reduced considerably compared to the initial conditions. On August 2, 2005, APT loadings of the rehabilitated Section n1 were renewed. Between August 2, 2005, and April 4, 2006, an additional 250,000 passes, all with wander, were applied to the section. Trafficking was discontinued because of time constraints. Very little cracking was reportedly seen on the pavement surface by that time. Both structural sections and the upper part of the subgrade were removed and discarded, along with the embedded 
instrumentation, so that another research project could be installed in the APT. This sequence of events is shown in Table 3.4.3.

Table 3.4.3: APT pass application log for Section n1 (rehabilitated structure).

\begin{tabular}{|c|c|c|c|}
\hline Date & Section & Cumulative Passes & Days \\
\hline \multicolumn{4}{|c|}{ Water introduced to subgrade beginning June $27^{\text {th }} 2005$} \\
\hline 2-Aug-05 & \multirow{35}{*}{ N1 } & $1,000(w)$ & 0 \\
\hline 15-Aug-05 & & $3,000(w)$ & 13 \\
\hline 16-Aug-05 & & $4,000(\mathrm{w})$ & 14 \\
\hline 17-Aug-05 & & $5,000(w)$ & 15 \\
\hline 18-Aug-05 & & $8,000(w)$ & 16 \\
\hline 22-Aug-05 & & $10,000(\mathrm{w})$ & 20 \\
\hline 31-Aug-05 & & $11,000(\mathrm{w})$ & 29 \\
\hline 1-Sep-05 & & $13,000(w)$ & 30 \\
\hline 6-Sep-05 & & $17,500(w)$ & 35 \\
\hline 7-Sep-05 & & $20,000(w)$ & 36 \\
\hline 26-Sep-05 & & $25,000(w)$ & 55 \\
\hline 3-Oct-05 & & $35,000(w)$ & 62 \\
\hline 24-Oct-05 & & $35,000(w)$ & 83 \\
\hline 1-Nov-05 & & $50,000(\mathrm{w})$ & 91 \\
\hline 2-Nov-05 & & $55,000(\mathrm{w})$ & 92 \\
\hline 7-Nov-05 & & $60,000(w)$ & 97 \\
\hline 14-Nov-05 & & $70,000(w)$ & 104 \\
\hline 15-Nov-05 & & $75,000(w)$ & 105 \\
\hline 22-Nov-05 & & $80,000(w)$ & 112 \\
\hline 28-Nov-05 & & $85,000(w)$ & 118 \\
\hline 29-Nov-05 & & $90,000(\mathrm{w})$ & 119 \\
\hline 5-Dec-05 & & $95,000(w)$ & 125 \\
\hline 6-Dec-05 & & $100,000(\mathrm{w})$ & 126 \\
\hline 12-Dec-05 & & $105,000(\mathrm{w})$ & 132 \\
\hline 13-Dec-05 & & $110,000(w)$ & 133 \\
\hline 19-Dec-05 & & $120,000(\mathrm{w})$ & 139 \\
\hline 3-Jan-06 & & $125,000(\mathrm{w})$ & 154 \\
\hline 9-Jan-06 & & $130,000(\mathrm{w})$ & 160 \\
\hline 16-Jan-06 & & $140,000(w)$ & 167 \\
\hline 23-Jan-06 & & $150,000(\mathrm{w})$ & 174 \\
\hline 24-Jan-06 & & $160,000(\mathrm{w})$ & 175 \\
\hline 6-Feb-06 & & $170,000(\mathrm{w})$ & 188 \\
\hline 13-Feb-06 & & $180,000(\mathrm{w})$ & 195 \\
\hline 27-Feb-06 & & $200,000(\mathrm{w})$ & 209 \\
\hline 4-Apr-06 & & $250,100(w)$ & 245 \\
\hline
\end{tabular}




\subsubsection{Identification of Dataset for Structural Investigation}

In general terms, this research aims at devising a method for applying APT results to field conditions. As put forward in Chapter 1: (i) the scope is limited to the case of duplicate pavement systems; (ii) the work plan consists of calibrating a mechanistic model to APT conditions and extending it using laboratory data; and (iii) the extended model is to be validated using NCAT results. Accordingly, it is argued that Section n1 dataset, collected in the APT between July and August 2004 (see Table 3.4.1), is best suited for carrying out structural investigation and achieving the main study objective. First and foremost, there is maximum similarity between this section and its replicate at NCAT, especially in the initial part of the experiment when both pavements were in their pristine state. This similarity was severely 'damaged' during the second round of n1 testing that took place between August 2005 and April 2006 (see Table 3.4.3), mainly because of the 'artificial' subgrade weakening and also because $\mathrm{n} 1$ became a rehabilitated structure: the surface HMA was replaced after incurring about 90,000 load passes and then the structure was allowed to rest/heal without traffic for almost a year before more loads were applied. Second, in this dataset APT passes were applied without wander, which means that the exact carriage position relative to the embedded gauges is known and available. This information is critical for model calibration. Finally, the construction operations are well documented and supplemented by laboratory tests.

This state of affairs is not the case for Section n2. First, the original surface HMA lift was replaced but the construction data and properties of the new mix are not available. Second, loading of n2 between September 2004 and February 2005 included wheel wander for which the exact carriage position was recorded only in the loading direction but not laterally. When the exact position of the loading is unknown, the approach followed herein fails because the APT model cannot be calibrated using inverse analysis. Finally, the main difference between sections $\mathrm{n} 1$ and $\mathrm{n} 2$ (or equivalently between $\mathrm{N} 1$ and N2) is the binder type. However, the master curves for n2/N2 mixes 3 and 4 were very similar to the n1/N1 master curves for mixes 1 and 2 (respectively). Hence, the resilient response of the two sections should also be very similar. In this connection, see also the FWD results in Figure 3.3.8. Moreover, as will 
be shown in the following section, large differences were recorded between gauges installed in Section $\mathrm{n} 1$ that were expected to measure identical responses. These differences are assumed to originate from structural heterogeneity and slight dissimilarity in gauge installation conditions. Consequently, any inherent dissimilarity in the response to load of the two sections is masked by these differences.

In summary, and based on the aforementioned sequence of events, the experimental dataset obtained during loading of Section $\mathrm{n} 1$ in the APT between July and August 2004 is selected for pursual of the study objectives. Although in Chapter 4 this dataset will be analyzed twice, for the pavement in its initial condition and also after 80,000 passes, the forecasting of NCAT response will focus on the initial loading phases during which the two experiments were most closely linked.

\subsection{STRUCTURAL BEHAVIOR}

\subsubsection{Instrumentation}

Instrumentation types and placement techniques used in the APT study were similar to these in the NCAT study (see Chapter 2, section 2.4). Pressure cells (Model 3500 manufactured by Geokon) were used as vertical stress gauges; CTL Group gauges (Model ASG-152) were used for measuring horizontal strains at the bottom of the HMA. These gauges were checked for functionality before and after embedment. The achieved level of survivability was $100 \%$. For further details refer to the reports included in Appendix C.

From a mechanical point of view, the introduction of a gauge in a pavement system produces changes to the stress and strain fields which influence the response of the pavement in the vicinity of the gauge and hence influence the recorded values. This disturbance was mostly investigated for the case of pressure cells (e.g., Tory and Sparrow, 1967; Brown, 1977; Tabatabaee and Sebaaly, 1990). Numerous factors have been identified that affect the measurements of pressure cells, including the ratio of cell thickness to diameter, the ratio of medium stiffness to cell stiffness, cell size, and field placement effects (Weiler and Kulhawy, 1982; Dunnicliff, 1988). 
Early synthetic work by Taylor (1945) and Monfore (1950) have shown that measurement errors can be reduced by minimizing the thickness to diameter ratio of the cell and making it as incompressible as possible. Based on experimental work, Peattie and Sparrow (1954) have shown that if these criteria are fulfilled then the measurement error relative to the 'true' stress level (in percent) equals 0.6 times the thickness to diameter ratio. Geokon earth pressure cells are classified as hydraulic type gauges; by design they have a thickness to diameter ratio of 0.026 (=6/230 see Figure 2.4.2) and are relatively incompressible. According to Peattie and Sparrow (1954), they are expected to record pressures that are higher than the 'true' stress levels by about $1.6 \%$. This is a relatively low error level for a geotechnical application.

More recently calibration chambers have been proposed as means for pressure cell calibration (Theroux et al., 2001; Labuz and Theroux, 2005). For pavement applications a more feasible method of calibration would be to apply a known load to the pavement at increasing distances from the gauge and calculate the resulting 'volume' of stresses. In theory this 'volume' should equal the applied load. This in situ type of calibration is best done with a single tire since the shape of the stress trace is symmetric and thus only a few measurement points need to be considered. In the current study dual-tires were used which resulted in an asymmetric stress trace. Also, the location of the tires relative to the gauge array was only measured in the longitudinal direction and not laterally. Hence it was not possible to perform such calibration herein. Similar reasoning precludes in situ calibration of the pressure cells for the NCAT study. With respect to the strain gauges, analysis of near field strain disturbance and resulting measurement errors could not be found in the literature. Subsequently, the stress and strain gauge readings were used as-is without applying any correction or calibration factors. In addition, it should be mentioned that both types of gauges experience drift over time due to temperature sensitivity (see Tesarik et al., 2006) and other reasons; this makes them suitable for capturing dynamic responses only and not for monitoring permanent changes.

A plan showing the embedded instrumentation aimed at capturing mechanical responses in test section $\mathrm{n} 1$ is provided in Figure 3.5.1. The loading centerline is denoted in the figure by the $\mathrm{Y}$-axis and the transverse direction by the $\mathrm{X}$-axis. The 
loading direction was from left to right along the Y-axis as indicated by the arrow. The entire gauge array is seen to be located in an eight foot (2.44 m) long strip, $2 \mathrm{ft}(0.61 \mathrm{~m})$ wide, in the central part of the test section. The first and last $6 \mathrm{ft}(1.83 \mathrm{~m})$ of the test section were not instrumented because the loading speed in these zones is not constant, with the carriage either accelerating or decelerating. In the central strip the loads are applied at a constant speed, which, in this study, was always $5 \mathrm{mph}(\sim 2.2 \mathrm{~m} / \mathrm{s})$. With respect to the $\mathrm{X}$ and $\mathrm{Y}$ axes in Figure 3.5.1, Table 3.5.1 lists the location of each gauge.

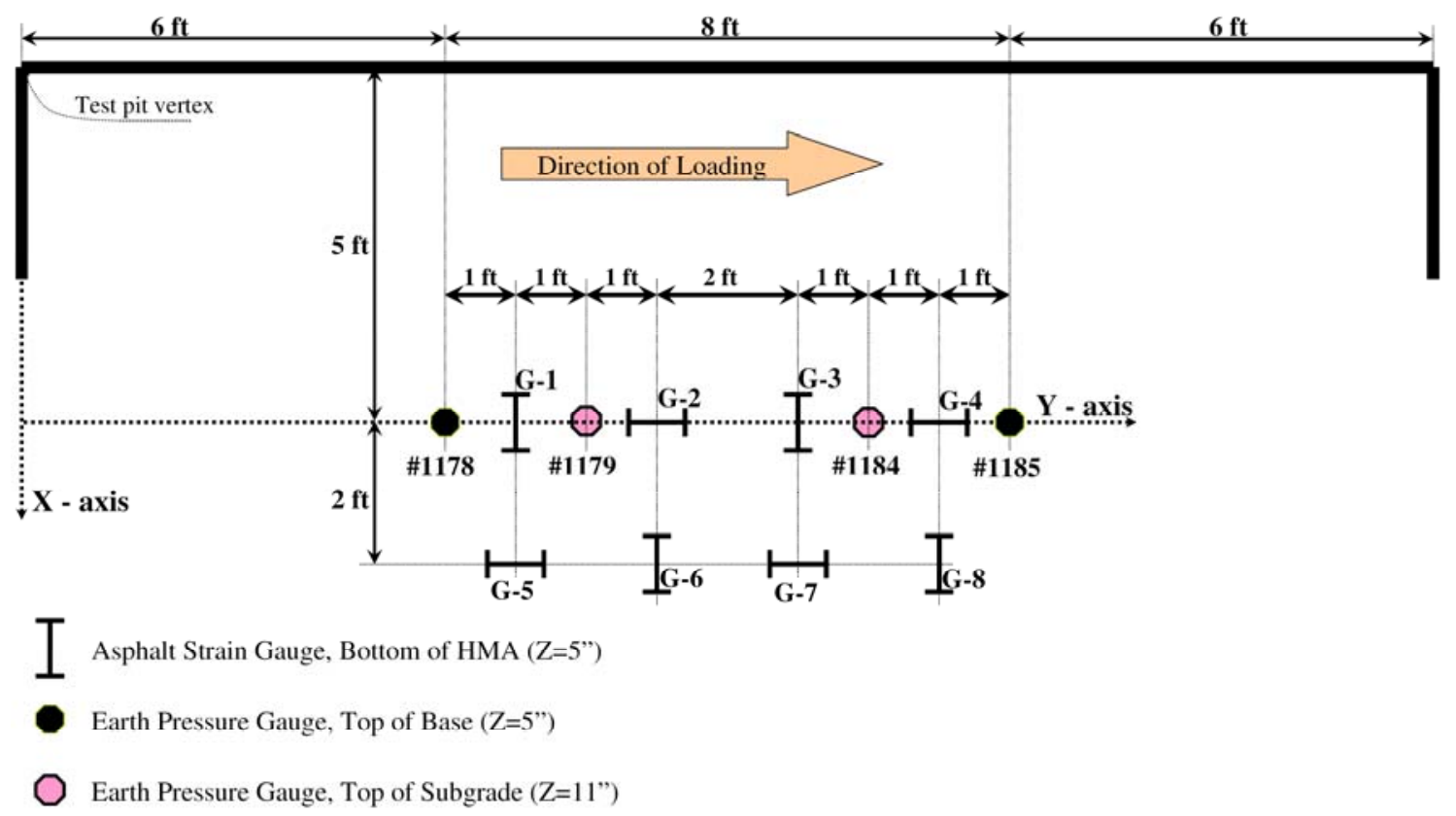

Figure 3.5.1: Plan of embedded instrumentation in APT lane 1 (Section n1).

As can be seen in Figure 3.5.1 (and Table 3.5.1), the pavement system was instrumented with a total of 12 gauges consisting of four pressure gauges and eight strain gauges. The two pressure gauges (\#1178 and \#1185) were measuring vertical stresses on top of the base course or bottom of the HMA. These were installed at a depth of 5 in. $(127 \mathrm{~mm})$ from the surface along the centerline of the loading path (i.e., Y-axis). Two additional pressure gauges (\#1179 and \#1184) were measuring vertical stresses on top of the subgrade or bottom of the base course. These were installed at a depth of $11.0 \mathrm{in}$. (279.4 mm) from the surface, also along the centerline.

All eight strain gauges were attached to the bottom of the HMA, i.e., at a depth of 5 in. (127 mm) from the surface. Gauges G-1, G-2, G-3 and G-4 were located along 
the centerline of the loading path. Strain gauges G-5, G-6, G-7 and G-8 were located along a parallel line positioned two feet $(0.61 \mathrm{~m})$ from the loading path. Gauges $\mathrm{G}-2$, G4, G-5 and G-7 were measuring horizontal strains in the loading direction (i.e., strain in Y) while gauges G-1, G-3, G-6 and G-8 were measuring horizontal strains in the transverse direction (i.e., strain in $\mathrm{X}$ ).

Table 3.5.1: Location of APT instrumentation in Section n1 (relate to Figure 3.5.1).

\begin{tabular}{|c|c|c|c|c|}
\hline Gauge ID & Gauge Type & $\begin{array}{l}\text { Location in } X, \\
\text { in. }(\mathrm{m})\end{array}$ & $\begin{array}{l}\text { Location in } Y, \\
\text { in. (m) }\end{array}$ & $\begin{array}{c}\text { Depth in } Z \text {, } \\
\text { in. }(\mathrm{mm})\end{array}$ \\
\hline$\# 1178$ & \multirow{4}{*}{ 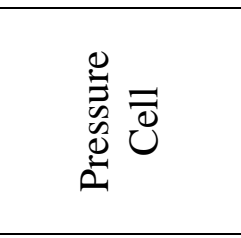 } & \multirow{8}{*}{0.0} & $72(1.83)$ & 5 (127) \\
\hline \# 1179 & & & $96(2.44)$ & \multirow{2}{*}{11 (279) } \\
\hline \#1184 & & & 144 (3.66) & \\
\hline$\# 1185$ & & & $168(4.27)$ & \multirow{9}{*}{5 (127) } \\
\hline G-1 & \multirow{8}{*}{ 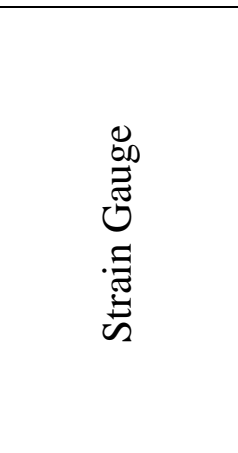 } & & $84(2.13)$ & \\
\hline G-2 & & & $108(2.74)$ & \\
\hline G-3 & & & $132(3.35)$ & \\
\hline G-4 & & & 156 (3.96) & \\
\hline G-5 & & \multirow{4}{*}{$24(6.1)$} & $84(2.13)$ & \\
\hline G-6 & & & $108(2.74)$ & \\
\hline G-7 & & & $132(3.35)$ & \\
\hline G-8 & & & $156(3.96)$ & \\
\hline
\end{tabular}

\subsubsection{Resilient Response}

The resilient response data presented and discussed hereafter was obtained from Section n1 during testing that took place between July and August 2004 (see Table 3.4.1 and discussion in Subsection 3.4.2). Recall that the loading was stopped after 90,000 passes due to bond failure that occurred at the interface between the surface and intermediate HMA lifts. Figures 3.5.2, 3.5.3 and 3.5.4 show the resilient strains and stresses measured during APT passes 5,000 and 80,000 vs. the APT carriage location which corresponds to the $\mathrm{Y}$-axis in Figure 3.5.1. In each of these figures, a solid line represents pass \#5,000 and a dashed line represents pass \#80,000. As is customary for geomaterials, a positive sign indicates compression and a negative sign indicates tension of either stress or strain. 
Figure 3.5.2 presents the vertical stresses measured on top of the subgrade and on top of the base course by the four pressure gauges. As can be seen, the resulting curves are bell-shaped and nearly symmetric. For loading pass \#5,000, peak vertical stresses on top of the base course were 30 and 35 psi (0.21 and $0.24 \mathrm{MPa})$. On top of the subgrade, the measured peak stresses were 16 and 20 psi (0.11 and $0.14 \mathrm{MPa})$. In theory the readings of each gauge pair should be identical. Furthermore, it may be seen that peak vertical stresses during pass \#80,000 are slightly higher compared to pass \#5,000. The difference is more significant in both absolute and relative terms for the gauges located on top of the subgrade compared to those located on top of the base course. It should be noted that the stress peaks occur slightly after the APT carriage had passed over the gauges and moved further along by about 2 to 5 in. (51 to $127 \mathrm{~mm}$ ).

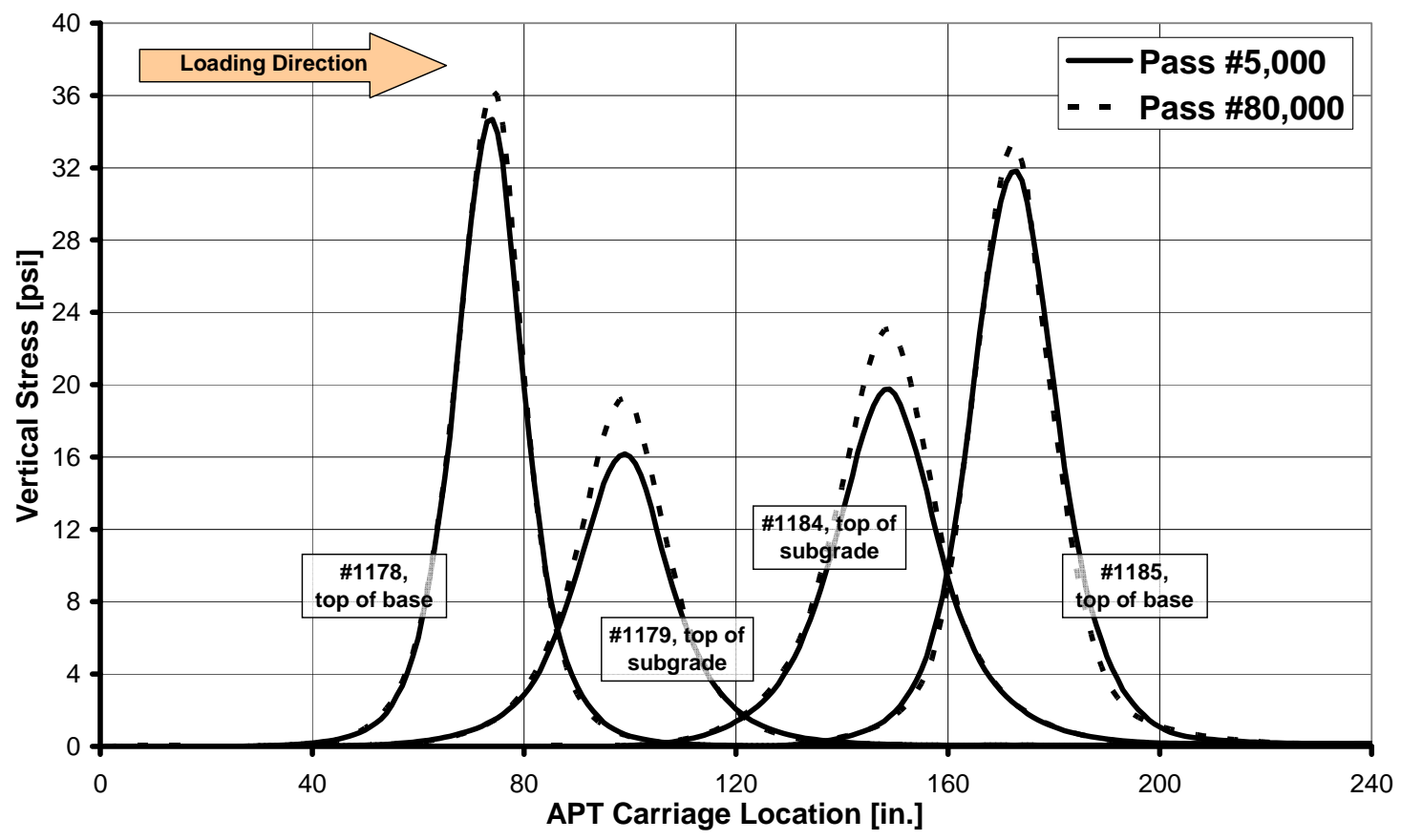

Figure 3.5.2: Measured vertical stresses in Section $\mathrm{n} 1$ on top of the base and on top of the subgrade during pass \#5,000 (solid line) and pass \#80,000 (dashed line).

Figure 3.5.3 presents the measured horizontal strains at the bottom of the HMA course in the direction of loading. Four gauge readings are shown, two of which were located along the loading centerline (G-2 and G-4), and two were located along a parallel line (G-5 and G-7) that is offset by two feet $(0.61 \mathrm{~m})$; see Figure 3.5 .1 and Table 3.5.1. In all four cases it can be seen that as the load approaches a gauge, the 
bottom of the HMA goes into compression. Then, the strain direction is reversed and the gauges go into tension. The point of maximum tension occurs when the APT carriage has passed the gauge positions along the $\mathrm{Y}$-axis by about 1 to 3 in. (25 to 76 $\mathrm{mm}$ ). Finally, when the load is receding (APT carriage moves further along), the tensile strains are reversed and compression is induced once more at the bottom of the HMA. This pattern is more pronounced for the gauges aligned along the centerline (G-2 and G4).

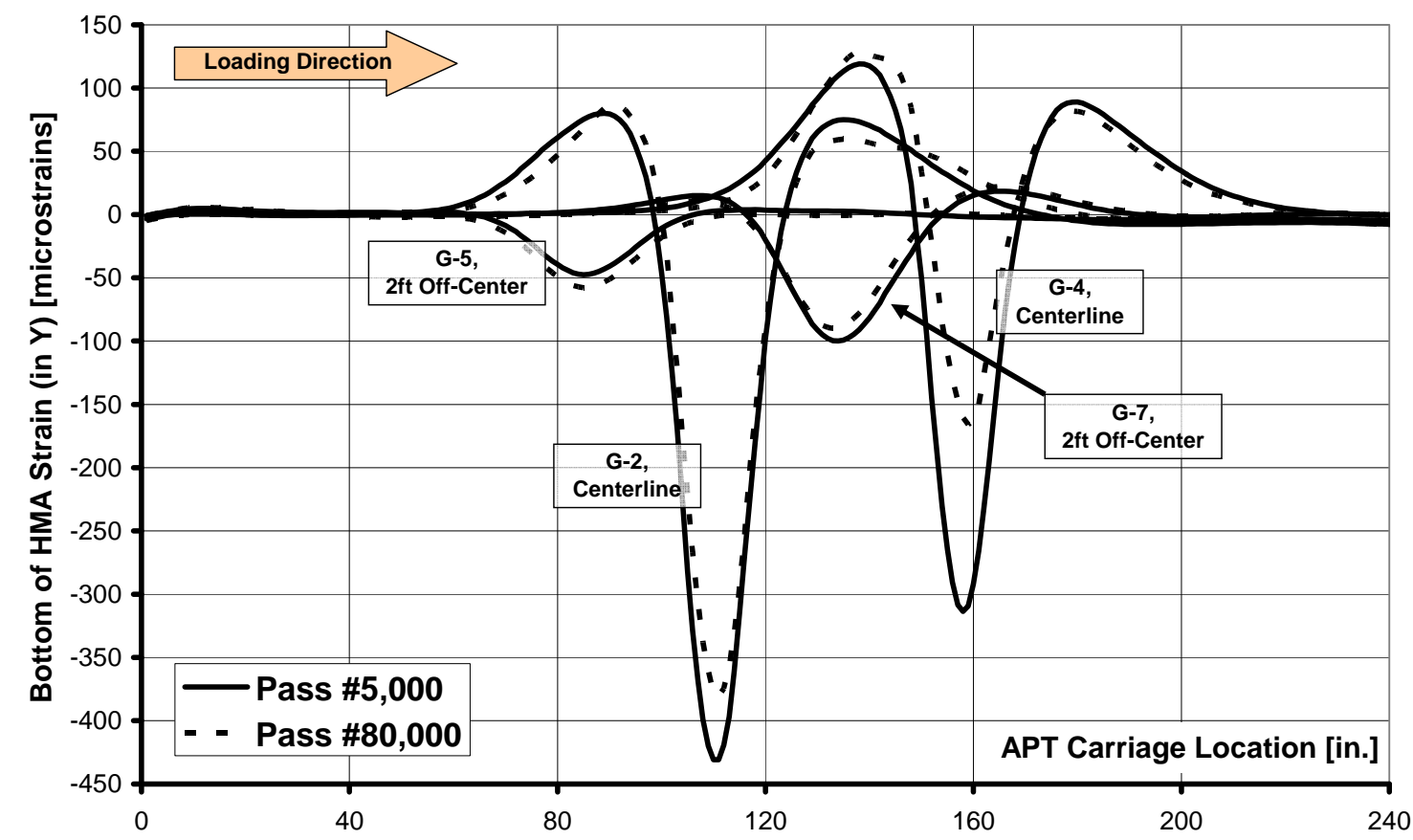

Figure 3.5.3 Measured horizontal strains at the bottom of the HMA in the direction of loading during pass \#5,000 (solid line) and pass \#80,000 (dashed line).

It can be graphically seen that the approaching branch of the strain response is different from the receding branch, resulting in a non-symmetrical time history curve. The two most noticeable differences are: (i) peak compressive strain is usually higher in the approaching branch compared to the receding branch; and (ii) the spacing along the $\mathrm{Y}$-axis between the tension and compression strain peaks is larger in the receding branch compared to the approaching curve.

Referring to the approaching branch of pass \#5,000 for gauges G-2 and G-4 (both centerline gauges), peak strains were 84 and 119 microstrains in compression and 
431 and 314 microstrains in tension (respectively). For gauges G-5 and G-7 (both offcenter gauges) the peak compressive stains were 2 and 15 microstrains while the peak tensile strains were 50 and 100 microstrains (respectively). In theory, the readings from each gauge pair should be identical. When comparing the response between pass \#5,000 and pass \#80,000 the most noticeable difference is seen in the peak tensile strain magnitudes for gauges G-2 and G-4 (the centerline gauges). For the G-2 strain gauge, peak strain in tension during pass $\# 80,000$ is 380 microstrains (compared to 431 microstrains during pass \#5,000). For G-4 gauge the peak strain in tension during pass $\# 80,000$ is 163 microstrains (compared to 314 microstrains during pass \#5,000).

Figure 3.5.4 presents the measured horizontal strains at the bottom of the HMA course in the transverse direction relative to the loading centerline. In this case the centerline gauges behave differently compared to the off-center gauges. Referring to pass \#5,000 data, it can be seen that gauges G-1 and G-3 (centerline gauges) go into tension as the load is approaching, with peak strains of 108 and 154 microstrains respectively. Contrary to the previous two figures, these peaks occur 4 to 6 in. (102 to $152 \mathrm{~mm}$ ) before the APT carriage reaches the gauge. As the APT carriage passes the gauges and moves further along, the strain direction is reversed until a small level of compression is induced. This compressive strain slowly recovers during the time period (not shown in the figure) in which the APT load is lifted from pavement and moved back to the startup position. Gauges G-6 and G-8 (off-center gauges) go into compression as the APT carriage approaches, with peak strains of 103 and 152 microstrains respectively. These peaks, however, occur 3 to 5 in. (76 to $127 \mathrm{~mm}$ ) after the load had passed each gauge. The receding branch of the response shows that the strain direction is reversed until a small level of tension is induced in the gauges.

The response of the gauges, as seen in Figure 3.5.4, is very confusing. First, the G-3 gauge shows two peaks instead of one as seen in the rest of the gauges. Next, when comparing the response between pass \#5,000 and pass \#80,000 the trends are not uniform: (i) it seems that the response of the G-3 gauge has shifted (delayed), as if the gauge was physically moved a few inches along the Y-axis during the experiment; and (ii) the peak strains decreased during the test for gauges G-1, G-3 and G-6 but not for 
the G-8 gauge. At this point we do not have a good explanation for these obscure behaviors.

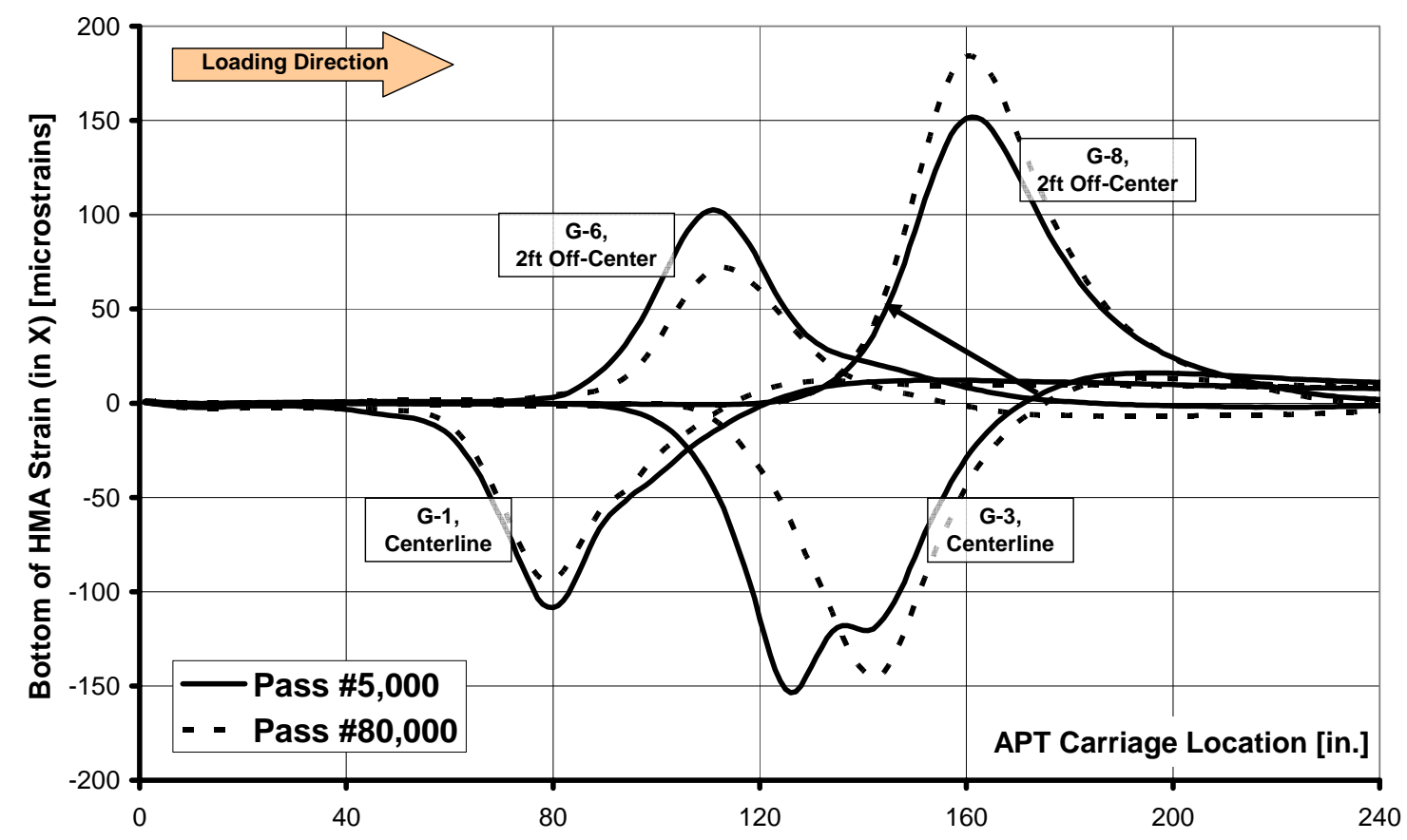

Figure 3.5.4: Measured horizontal strains at the bottom of the HMA in the transverse direction to the loading during pass \#5,000 (solid line) and pass \#80,000 (dashed line).

\subsubsection{Rutting and Cracking Performance}

Periodically, the APT carriage was halted for mapping of surface cracks and so that rutting measurements could take place. No cracking information is available from the APT study as cracks did not appear on the surface throughout the experiment. Reportedly, a very few hairline cracks did appear at the very end of the experiment in Section n1 (see Table 3.4.3). Their location and orientation, however, was not mapped before digging out the materials to make room for the following APT study.

The device for obtaining transverse profiles consists of a vertical rod with a small wheel attached to its tip. The rod is moved manually along a guided straight line across the pavement, with its rolling wheel in continuous contact with the surface. During this process both vertical and horizontal movements are collected providing about 680 data pairs. Horizontal position is measured with a cable-based transducer while the vertical position is measured using an LVDT. The entire device is attached to 
the overhanging APT beams that span the test pit thus providing a fixed reference for the measurements throughout the experiment. In an effort to assess the accuracy of the profiler, a nominally flat concrete surface was measured repeatedly along the same line. The standard deviation of readings was found to be $0.015 \mathrm{in}$. $(0.38 \mathrm{~mm})$. Referring to a single point on the pavement, the maximum difference between two individual profile readings taken at different times was found to be 0.069 in. $(1.75 \mathrm{~mm})$. On an average this difference was 0.048 in. $(1.22 \mathrm{~mm})$. More details on the profiler can be found in Huang (1995) and in Galal and White (1999). Table 3.4.1 shows when rutting profiles were taken in Section n1. As can be seen, the dates in the table correspond to the following cumulative number of APT passes (without wander): 0, 5k, 10k, 20k, 30k, 40k, 50k, 60k, 70k, 80k and 90k. In each case nine cross sections were determined, spaced $2 \mathrm{ft}(0.61 \mathrm{~m})$ apart. Corresponding to the $\mathrm{Y}$-axis in Figure 3.5.1, their locations were: 24, 48, 72, 120, 144, 168, 192, 216, and 240 in. (0.61, 1.22, 1.83, 2.44, 3.05, 3.66, 4.27, 4.88 and $5.49 \mathrm{~m}$ ). The complete profile data set can be found in Appendix A.

For illustration purposes, the profiles measured at the central cross section with $\mathrm{Y}=120$ in. (2.44 m) are shown in Figure 3.5.5. This figure shows 12 profiles relative to the initial profile. The magnitude of surface depression is shown on the ordinate on the left. The dashed horizontal line at zero rutting represents the pavement immediately before testing. The abscissa indicates the offset in the $\mathrm{X}$ direction relative to the loading centerline (refer to Figure 3.5.1). The results for passes 100, 500 and 1000 were obtained using interpolation assuming that the rutting increased linearly when APT passes \#1 to \#5000 are depicted on a logarithmic scale.

As can be seen in Figure 3.5.5, during the application of the first 60,000 passes the vertical surface displacement is seen to continuously increase. In absolute terms, a maximum surface depression of $0.45 \mathrm{in}$. (11.4 mm) was reached directly under the tires. With additional APT passes, this maximum is slightly reduced while surface heaving takes place outside the wheel path. First on the right side at about pass \#70,000 and then also on the left side during pass \# 90,000. 


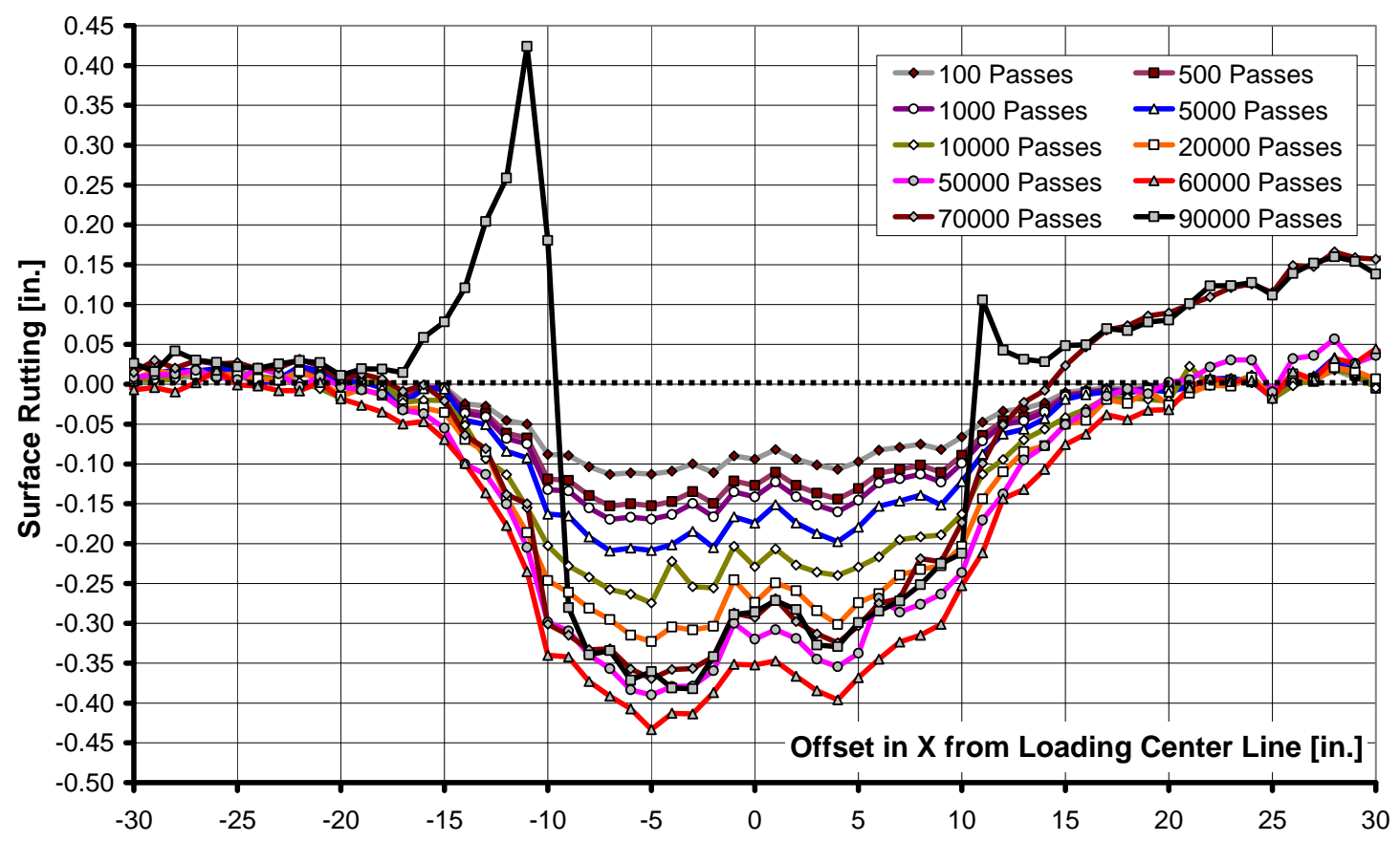

Figure 3.5.5: Rutting development in Section $\mathrm{n} 1$ at the central cross section during the first 90,000 load passes (applied without wheel wander).

Figures 3.5.6 to 3.5.18 contain contour charts (13 charts in total) that show the development of rutting in the APT. Each figure is basically a plan view of lane 1 with $X$ and $\mathrm{Y}$ axes as defined in Figure 3.5.1. The different colors (or shades) in the charts represent different rutting depths (legend is identical in all figures). In preparing these figures, use was made of all available profiles measured during the entire experiment; each figure represents a different pass level, shown in a box on the lower right corner; starting with pass \#100 (Figure 3.5.6) and ending at pass \#90,000 (Figure 17). The outcome helps visualize how rutting progressed under load. One immediate observation from these charts is that rutting was consistently deeper in the first half of the test lane (i.e., $\mathrm{Y}<144$ in.). This indicates structural heterogeneity that may, at least partially, explain the dissimilarity in readings from gauge pairs that should, in theory, be measuring identical response (see discussion in previous subsection). 


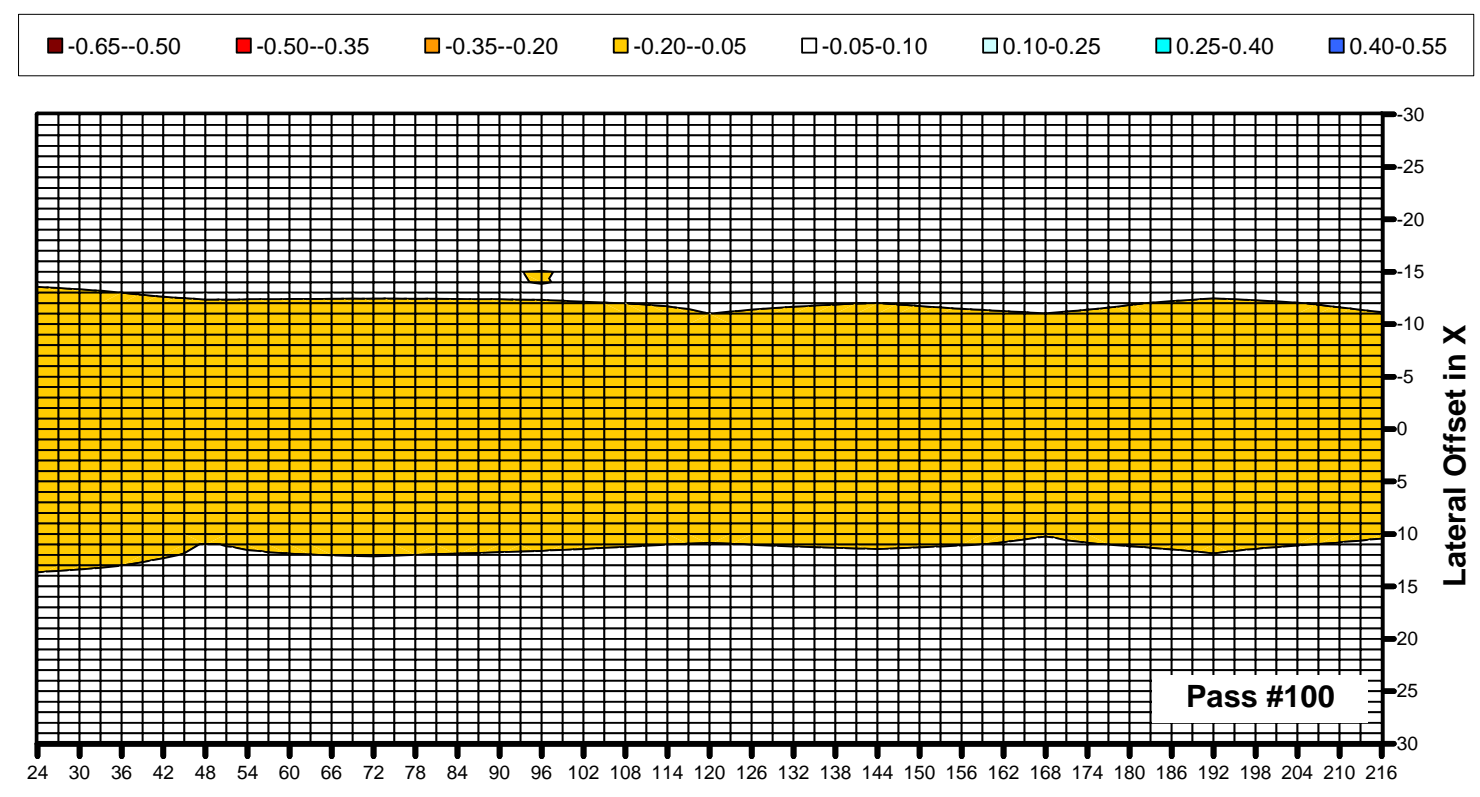

Location in $Y$ [in.]

Figure 3.5.6: Contour plot of Section n1 rutting after 100 passes.

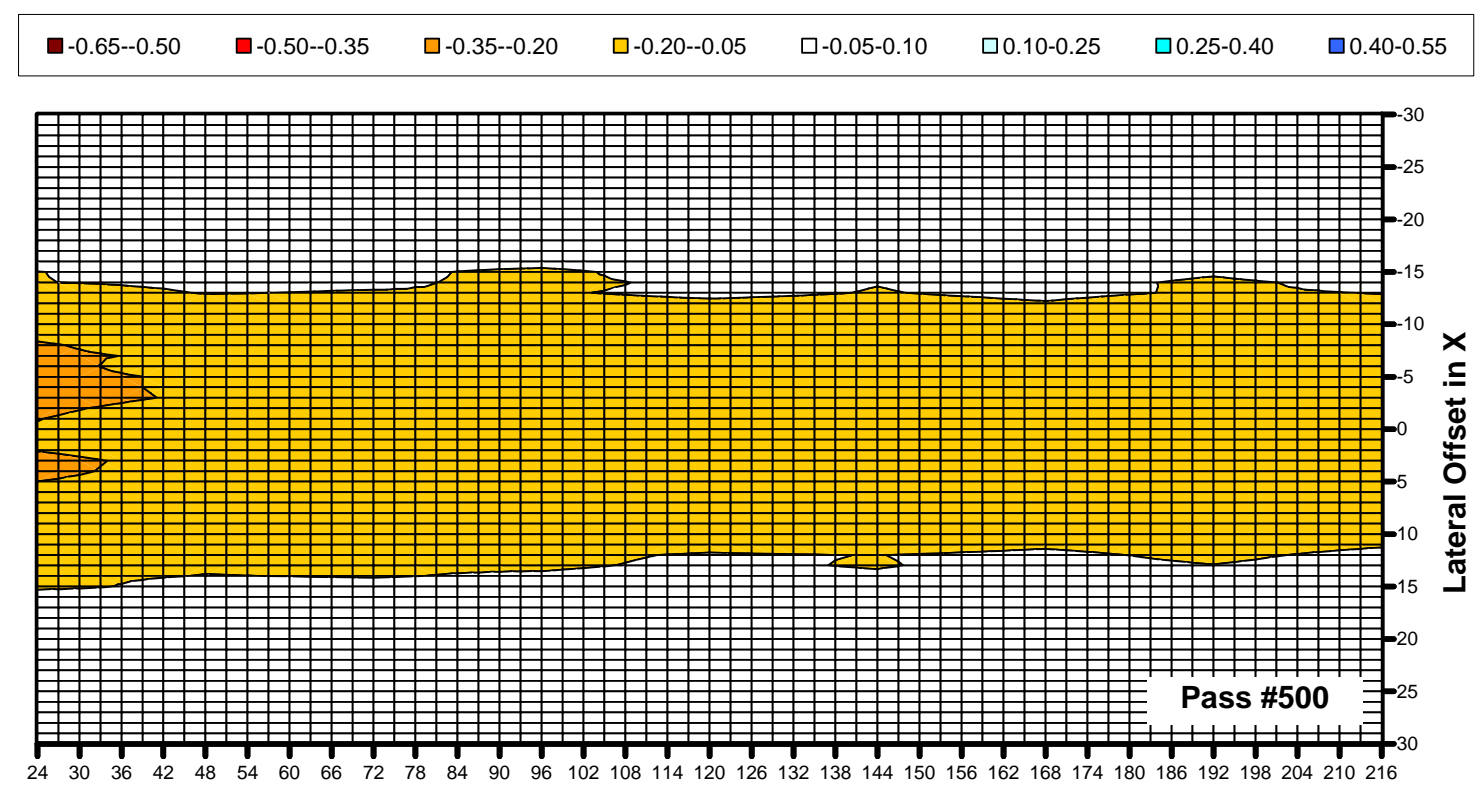

Location in $Y$ [in.]

Figure 3.5.7: Contour plot of Section n1 rutting after 500 passes. 


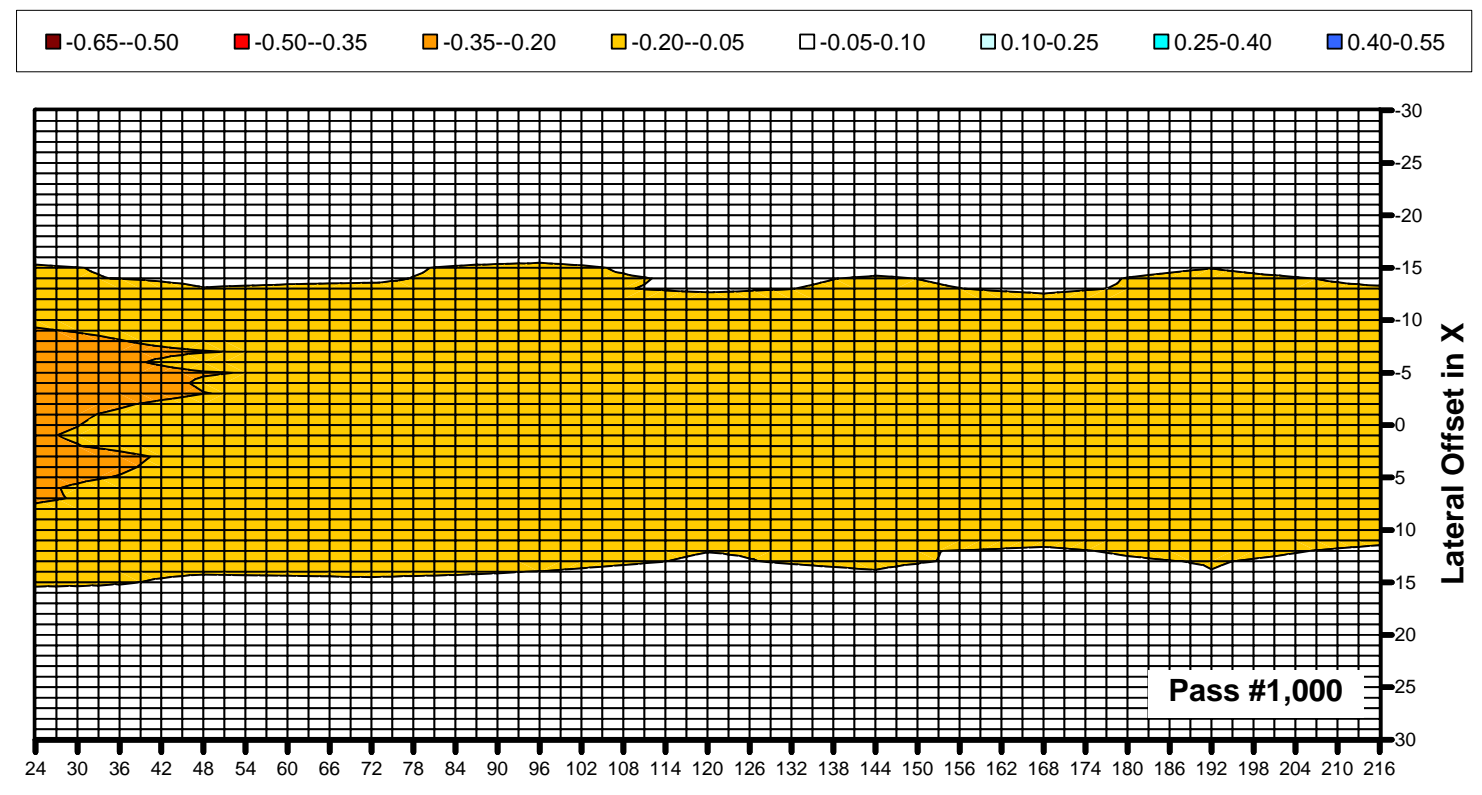

Location in $\mathrm{Y}$ [in.]

Figure 3.5.8: Contour plot of Section n1 rutting after 1,000 passes.

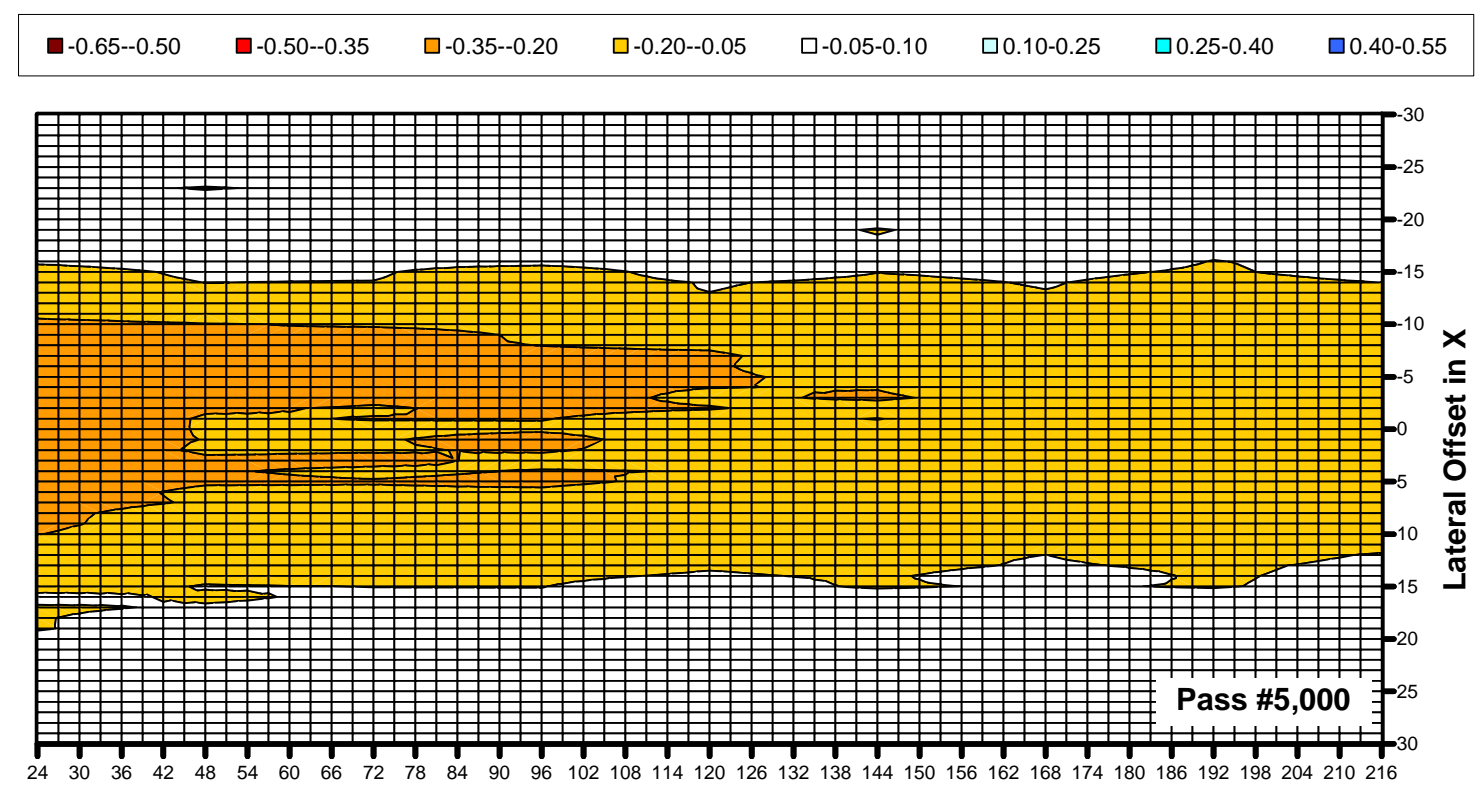

Location in $\mathrm{Y}$ [in.]

Figure 3.5.9: Contour plot of Section n1 rutting after 5,000 passes. 


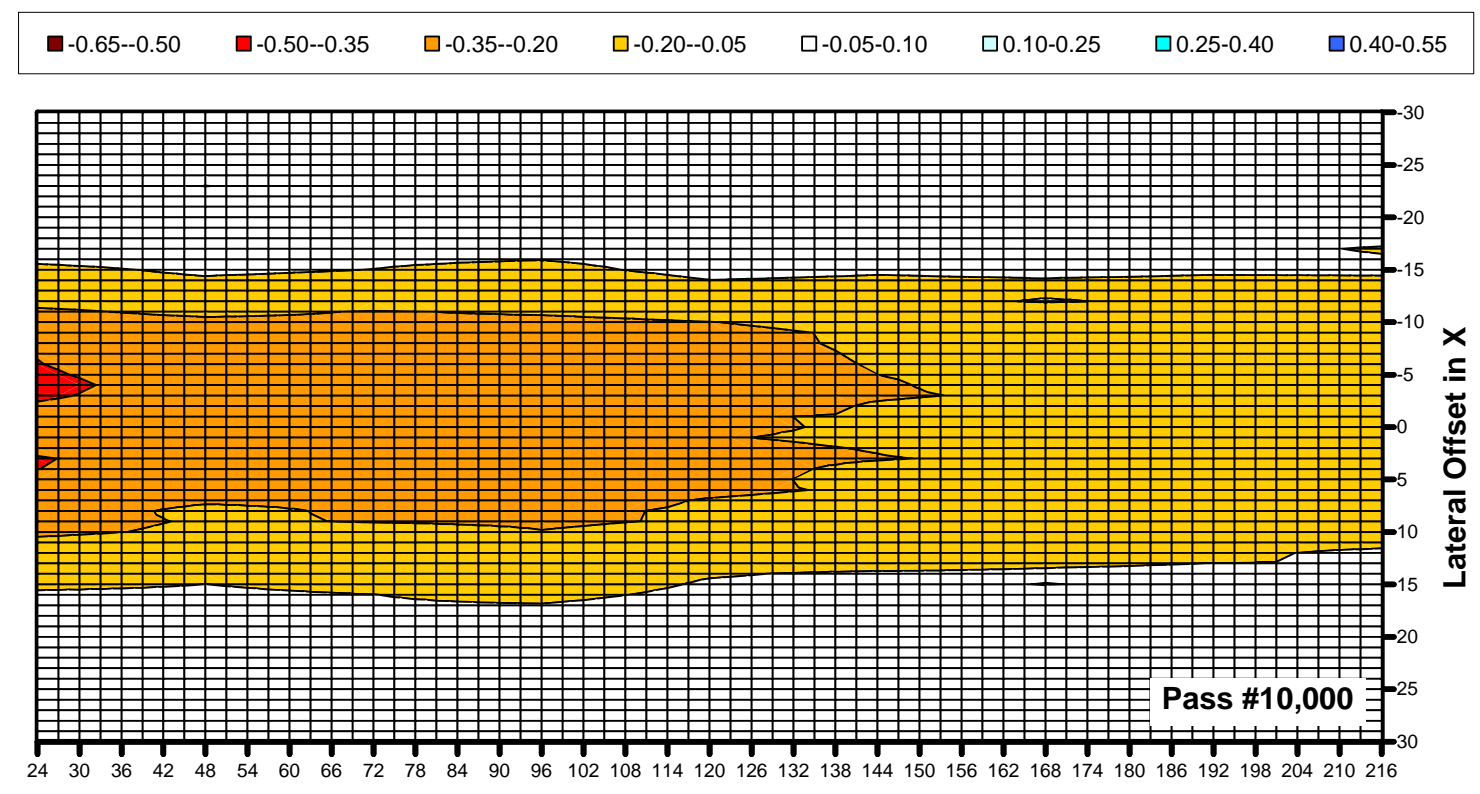

Location in $\mathrm{Y}$ [in.]

Figure 3.5.10: Contour plot of Section n1 rutting after 10,000 passes.

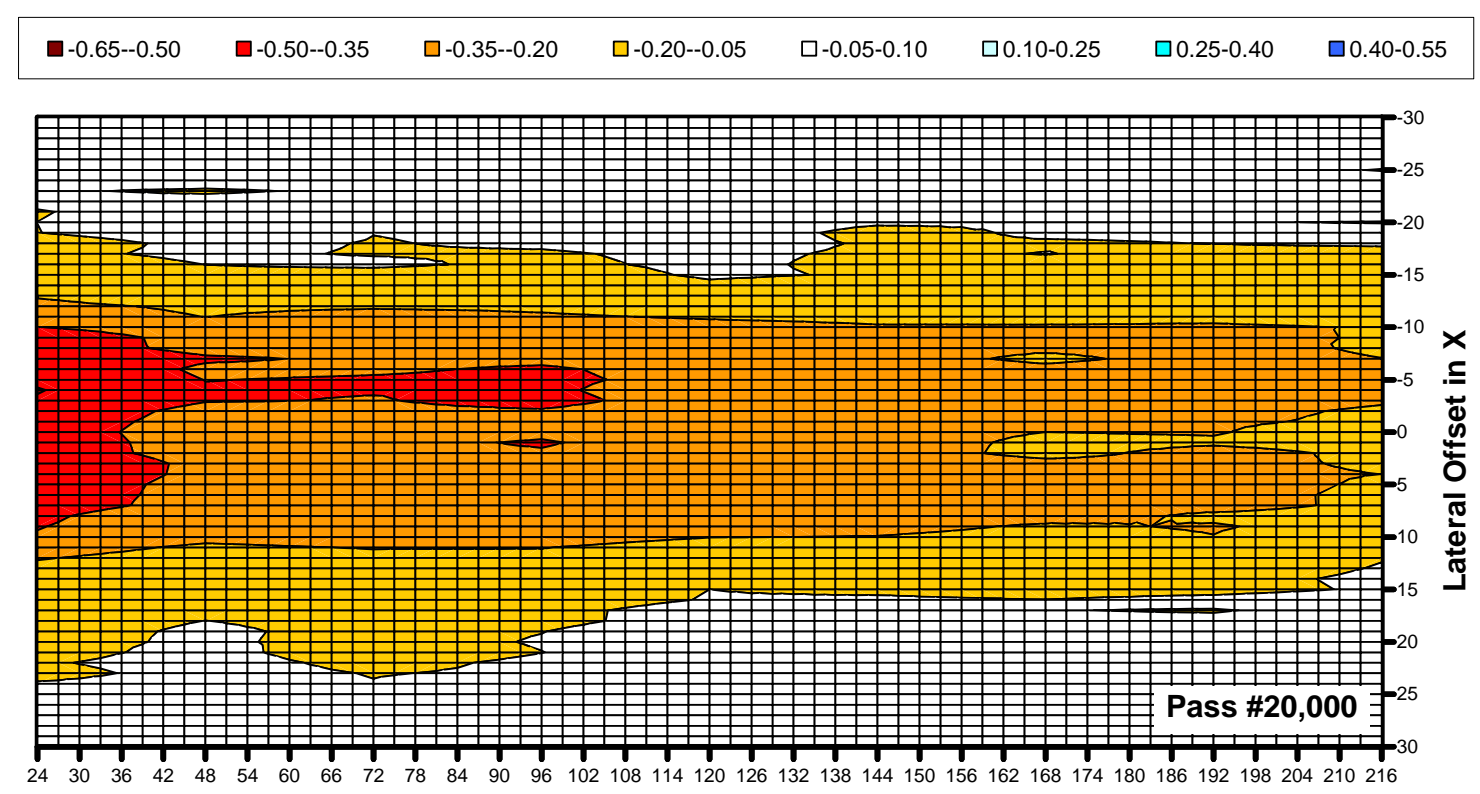

Location in $\mathrm{Y}$ [in.]

Figure 3.5.11: Contour plot of Section n1 rutting after 20,000 passes. 


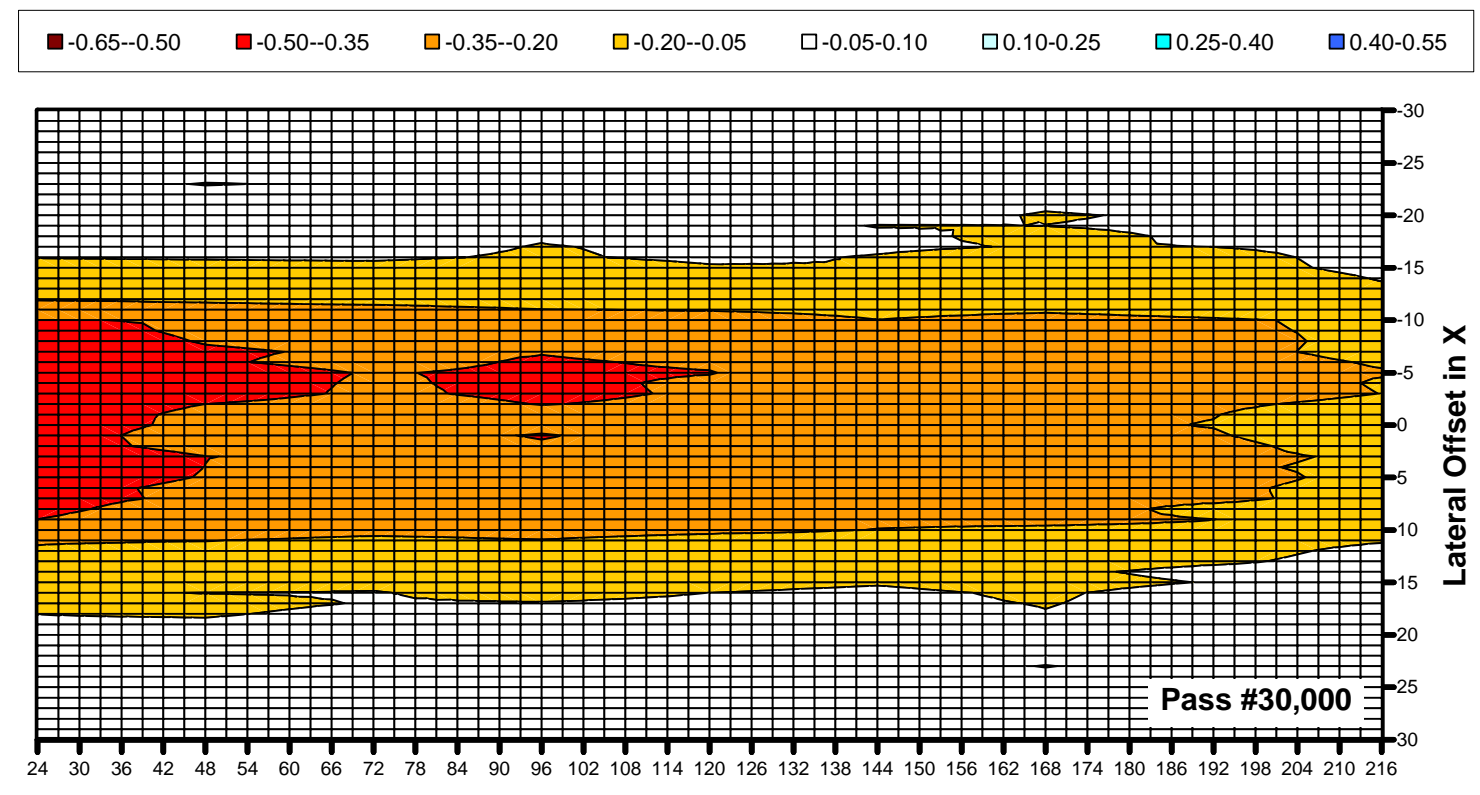

Location in $\mathrm{Y}$ [in.]

Figure 3.5.12: Contour plot of Section n1 rutting after 30,000 passes.

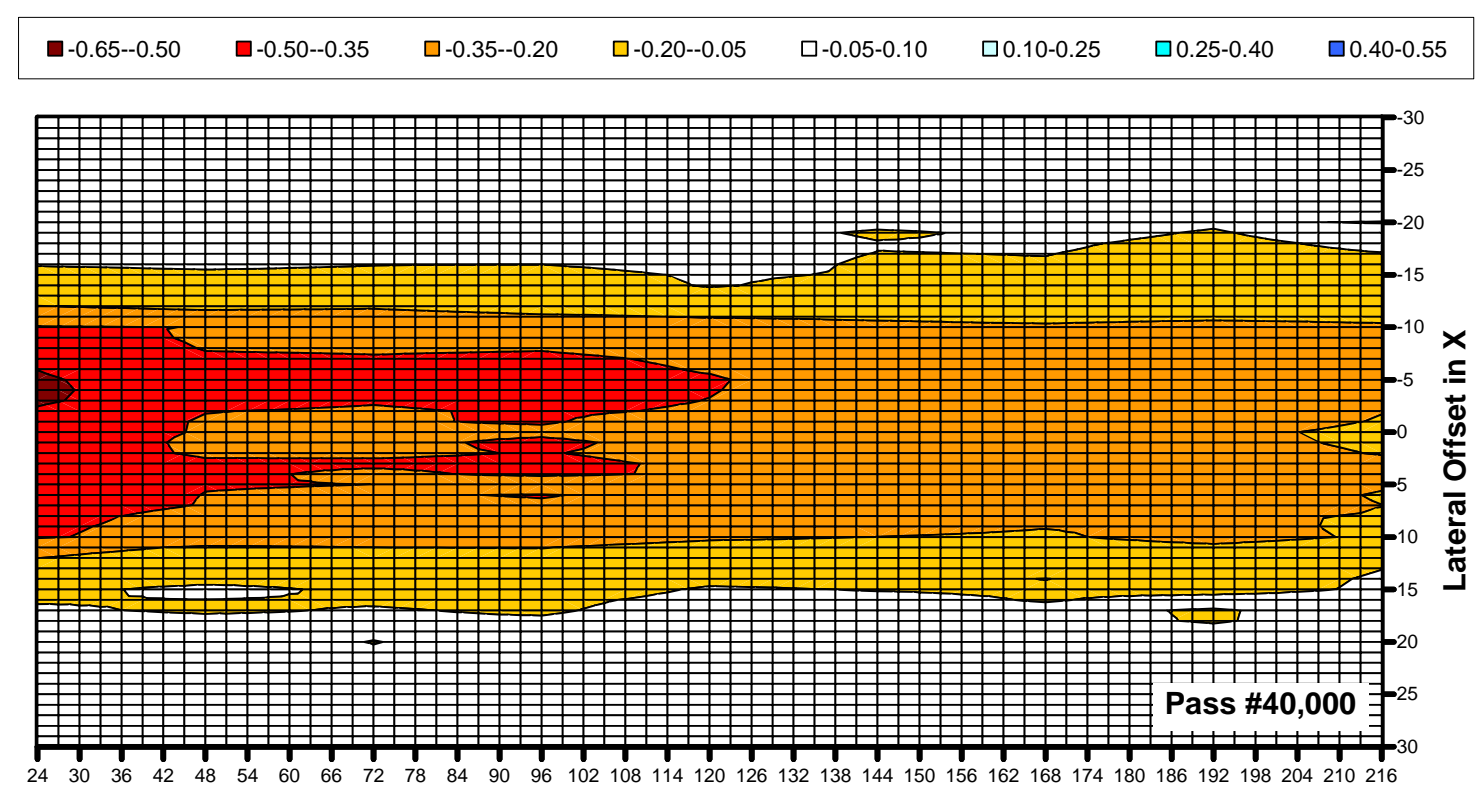

Location in $\mathrm{Y}$ [in.]

Figure 3.5.13: Contour plot of Section n1 rutting after 40,000 passes. 


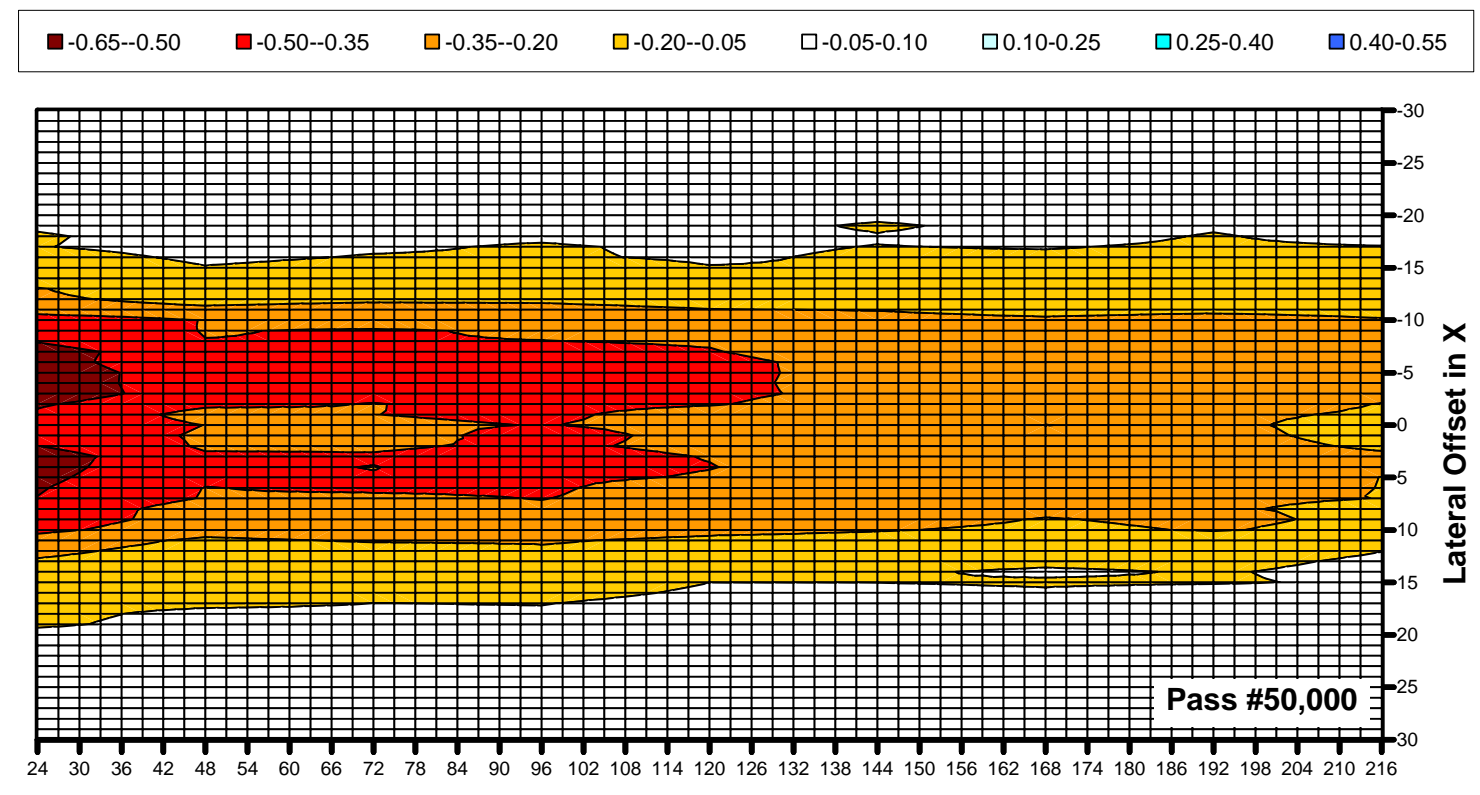

Location in $\mathrm{Y}$ [in.]

Figure 3.5.14: Contour plot of Section n1 rutting after 50,000 passes.

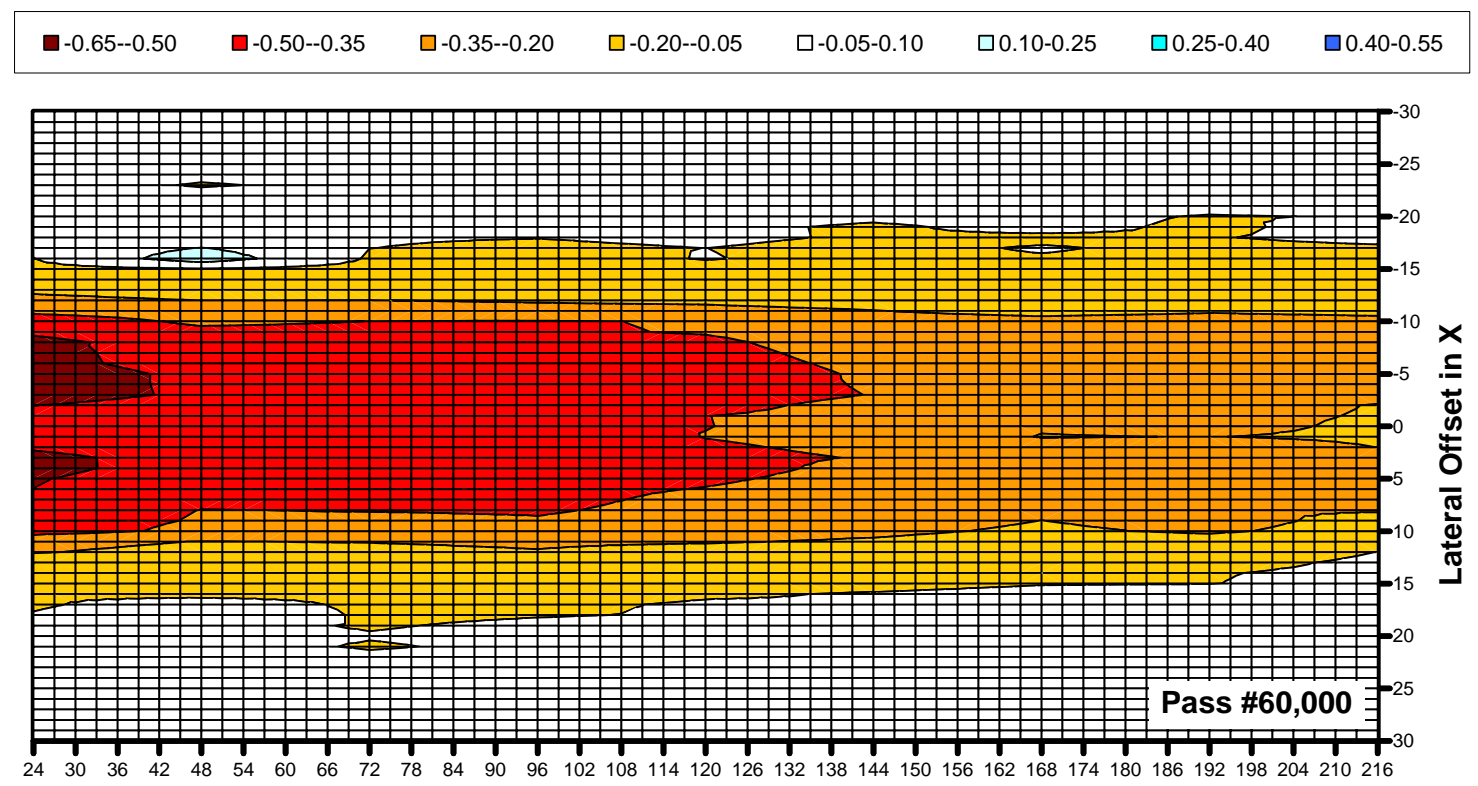

Location in Y [in.]

Figure 3.5.15: Contour plot of Section n1 rutting after 60,000 passes. 


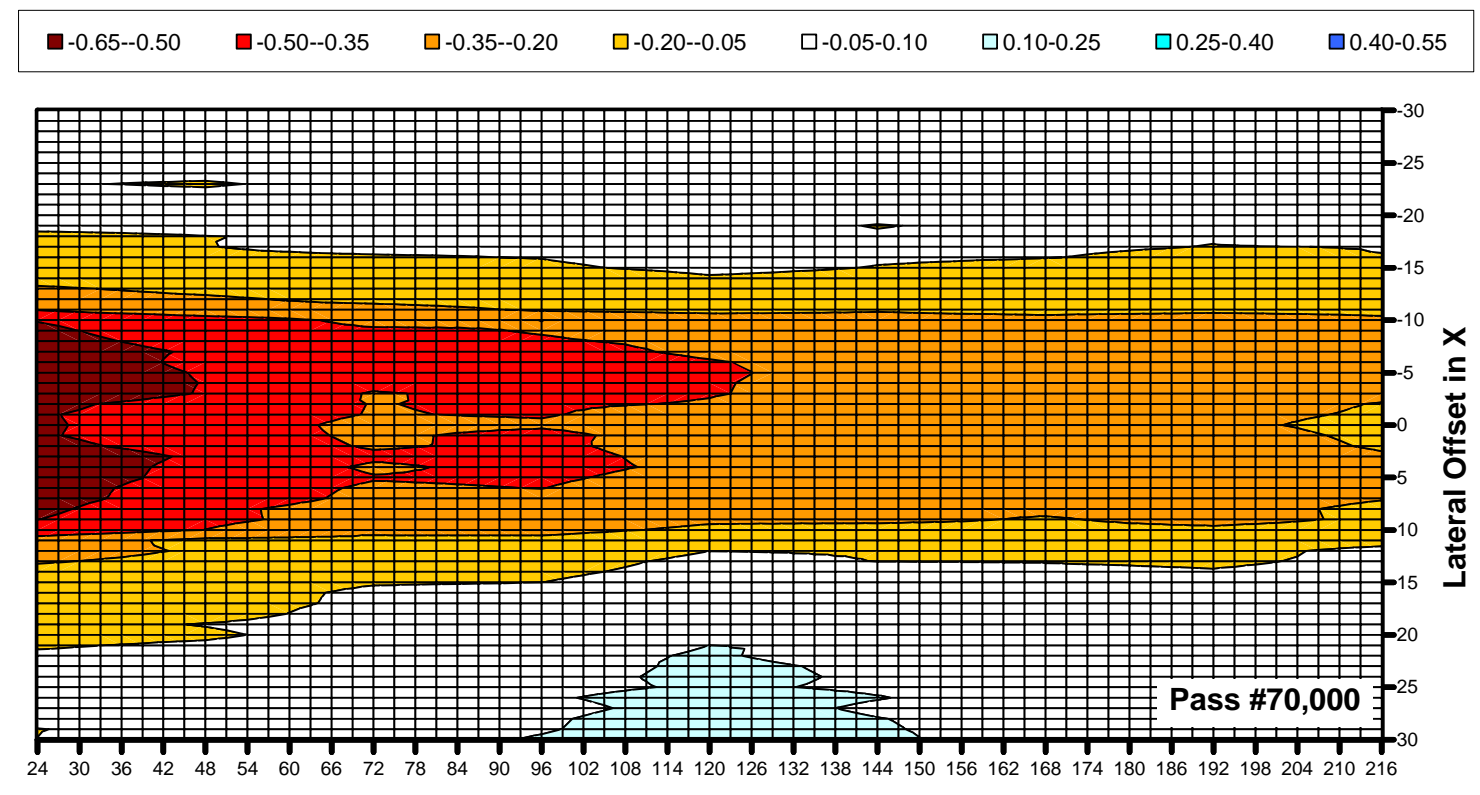

Location in $\mathrm{Y}$ [in.]

Figure 3.5.16: Contour plot of Section n1 rutting after 70,000 passes.

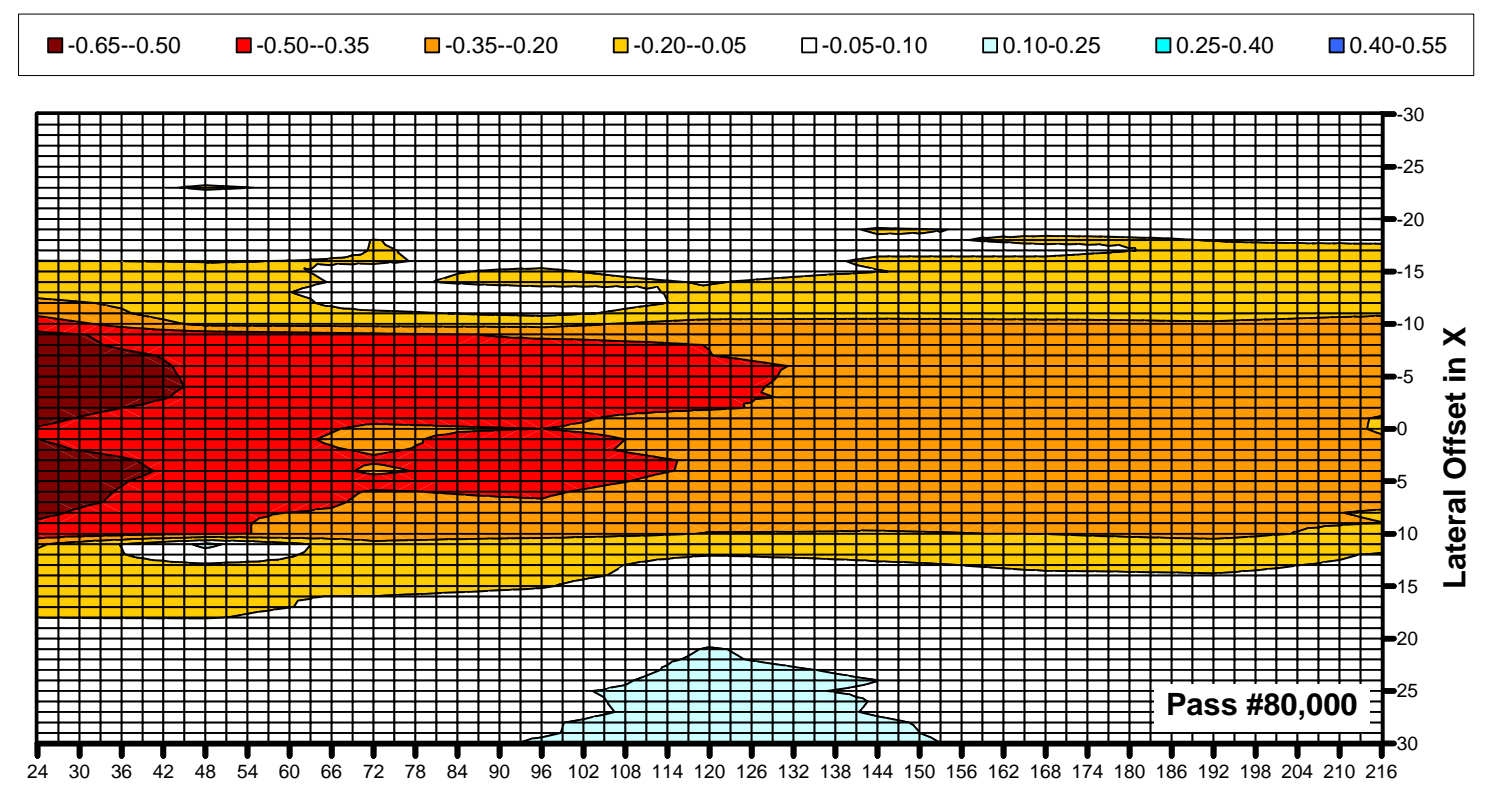

Location in $\mathrm{Y}$ [in.]

Figure 3.5.17: Contour plot of Section n1 rutting after 80,000 passes. 


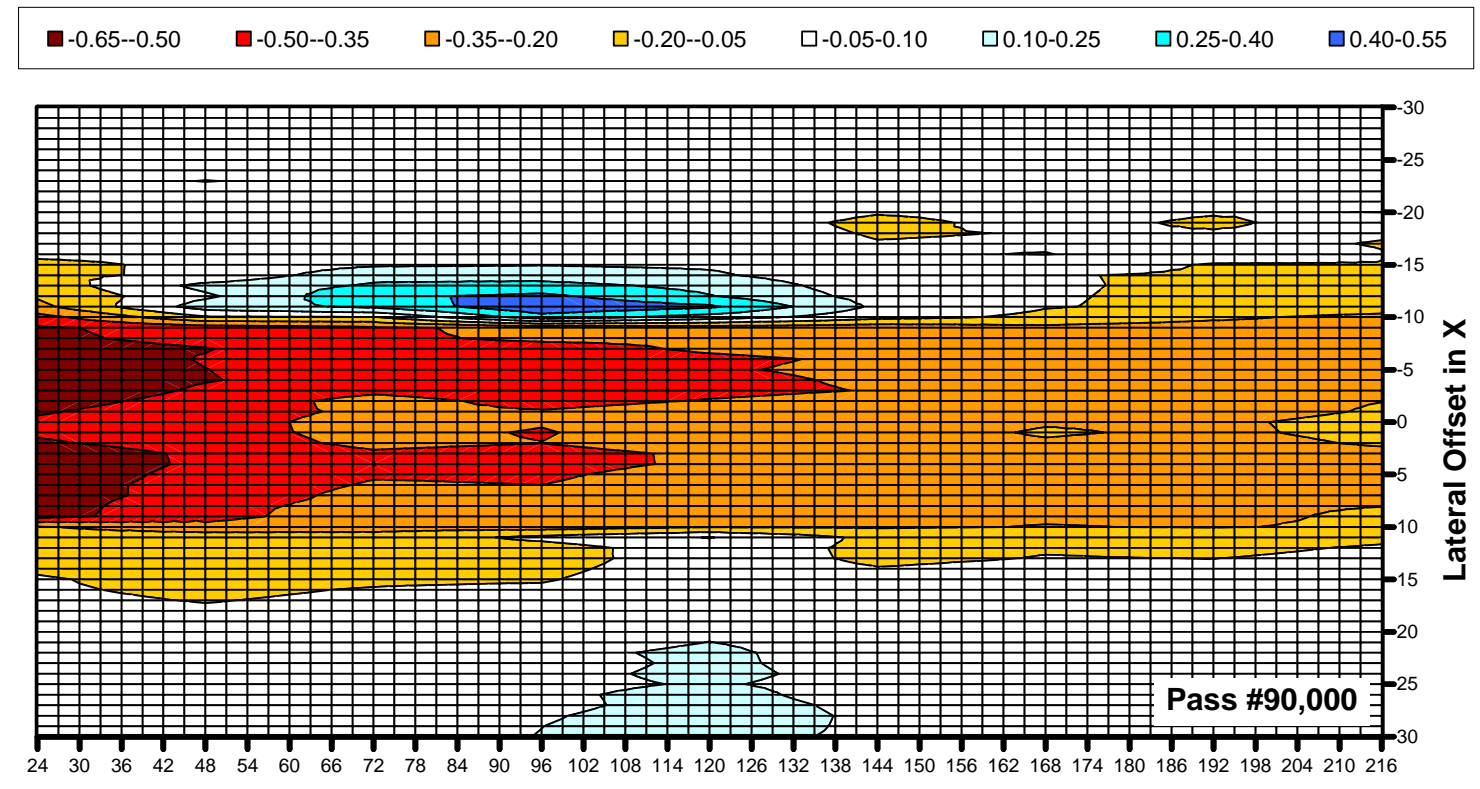

Location in $\mathrm{Y}$ [in.]

Figure 3.5.18: Contour plot of Section n1 rutting after 90,000 passes.

\subsection{DIRECT COMPARISON WITH NCAT RESULTS}

Assuming similar pavement systems were built at NCAT and in the APT, there should be a way to relate the two experiments. The simplest and most direct method of analysis is to compare the observed behavior by contrasting (separately) the observed performance and response.

In the NCAT study the N1 (and N2) pavement failed predominantly in fatigue mode (see Figure 2.5.5) while very little rutting was observed. The maximum recorded rut depth (Figure 2.5.6) was 0.31 in. (8 mm). In contrast, in the APT study very little (if any) cracking occurred, and the $n 1$ pavement experienced under the first 90,000 passes alone (see Table 3.4.1) maximum rutting of about 0.65 in. (11.5 mm) and heaving of similar magnitude (see Figure 3.5.18).

NCAT responses to one truck pass were shown in Figures 2.5.1 to 2.5.4. Each figure includes strain or stress pulses recorded by one gauge. As can be seen, one pass induced eight pulses with different peak magnitudes even for nominally identical axle loads. Also, the pavement was allowed to rest for 45 seconds between truck passes (assuming three running trucks) and for about two full days during weekends. APT 
responses were shown in Figures 3.5.2 to 3.5.4. In contrast to the NCAT case, each APT pass resulted in one stress (or strain) pulse; also, the pavement was allowed to rest for 8 seconds between passes. It should be noted that the APT was operated on weekends also. Graphically, the response traces from the two experiments are very much different and any attempt to directly compare them is futile given that they embody many dissimilarities:

(i) Loading Speed: $45 \mathrm{mph}$ at NCAT vs. $5 \mathrm{mph}$ in the APT. The loading speed influences the duration of stress and stress pulses and affects the HMA stiffness;

(ii) Axle Configuration: NCAT had three axle types, single, dual and dual tandem vs. one axle type in the APT, dual;

(iii) Axle Load: NCAT axles were loaded to 20,000 pounds except for the steer axle which was loaded to 10,000 pounds vs. one load level in the APT of 15,000 pounds;

(iv) Location of Loads relative to the Measuring Gauge: NCAT loads were applied near the gauge array but the exact location is unknown while in the APT study the load could be positioned accurately;

(v) HMA Temperature: NCAT temperature changed with the environment while it was constant in the APT at $15.5^{\circ} \mathrm{C}$. The prevailing temperature influences the stiffness of the HMA and hence the resulting responses.

Based on the above discussion it may be concluded that a direct relation between the two experiments cannot be established as both performance and response are distinct. Consequently a more fundamental approach is needed to link the observed behavior. This is pursued in the following chapters using mechanistic principals assuming the pavement systems in the two experiments have similar material properties. Mechanistic treatment can account for each of the aforementioned dissimilarities. As put forward and explained in Chapter 1 (Section 1.2) the analysis will focus on resilient responses. 


\section{CHAPTER 4 - BASIC MECHANISTIC ANALYSIS}

This chapter addresses the primary study objective by establishing a relation between the NCAT and APT experiments. The scope and approach are discussed in Section 4.1. Section 4.2 contains the development of a basic mechanistic model for the pavement systems considered; it is based on layered elastic theory (LET) with the necessary material properties obtained via inverse analysis of APT results. Section 4.3 deals with NCAT response prediction; the calculation methodology is first explained and then applied to forecast selected responses.

\subsection{SCOPE AND APPROACH}

In Section 3.6 it was argued that a fundamental approach is needed to link the NCAT and APT experiments. This is pursued hereafter focusing on load related resilient responses using isotropic LET. The APT experiment is first analyzed. The $\mathrm{n} 1$ pavement system is modeled using four isotropic layers (see Subsection 4.2.1) comprised of HMA, base and subgrade on top of a semi-infinite concrete medium. The latter represents the concrete floor present at the bottom of the APT pit. The unknown material properties (layer moduli) are obtained through backcalculation by matching the gauge readings collected during one pass of the APT carriage (see Subsection 4.2.2). Due to the temperature and rate sensitivity of the HMA, the resulting properties represent the environment and loading configuration in the APT only. This 'inverse analysis' is performed twice, for the pavement in the initial phases of the experiment, after 5,000 passes, and also later in the experiment after 80,000 passes. Pavement properties in both cases (i.e., layer moduli) are presented and compared (Subsection 4.2.3), showing that the structure experienced permanent property changes under the repetitive APT passes.

Next, the calibrated APT model is extended to apply to other loading configurations and environments. Assuming similarly constructed pavement systems, the methodology consists of changing the HMA modulus to reflect different loading speeds and temperatures. This is done after additional analysis of laboratory complex modulus results (see details in Subsection 4.3.1). The unbound material properties, 
although stress-state sensitive, are assumed unaffected by the changes in the HMA stiffness. This may be justified, at least as a first order approximation, considering that there are preexisting effective confining stresses in these materials (of unknown magnitudes) originating from the construction process; these include vertical stresses due to self weight, locked-in horizontal stresses from the compaction process, and confining stresses due to negative pore pressures (see Subsection 3.3.1).

Finally, the loading and environment at NCAT are simulated and the extended model is used in a forward calculation mode to forecast NCAT responses. Three types of resilient responses are investigated (subsections 4.3.2 and 4.3.3): (i) peak surface deflections observed during FWD testing (Subsection 4.3.2); (ii) vertical stresses on top of the subgrade and aggregate base caused by an NCAT truck; and (iii) horizontal strains at the bottom of the HMA induced by an NCAT truck. Owing to the permanent property changes occurring in the APT experiment, the analysis focuses on the pavement in the initial stages of the experiment. The concrete floor in the APT is left in place during the forward analysis because deeper into the NCAT subgrade a rigid bedrock material is expected (see Subsection 2.2.2).

\subsection{LAYERED ELASTIC ISOTROPIC MODEL}

\subsubsection{Theory and Computational Implementation}

Since its introduction by Burmister (1943; 1945), isotropic LET has been used by engineers and researchers for representing the load induced resilient responses of asphalt pavement systems. At this time, isotropic LET serves as the main 'engine' for the MEPDG through the JULEA computer code (Uzan, 1976). According to the theory, pavement materials are assumed to be linear elastic, homogeneous, isotropic and weightless, characterized by an elastic (Young's) modulus, E, and a Poisson's ratio, $v$.

Using a cylindrical coordinate system $(r, \theta, z)$, and assuming an axially symmetric deformation field, the constitutive law is:

$$
\varepsilon_{r}=\frac{1}{E} \cdot \sigma_{r}+\frac{v}{E} \cdot\left(\sigma_{\theta}+\sigma_{z}\right) .
$$




$$
\begin{aligned}
& \varepsilon_{\theta}=\frac{1}{E} \cdot \sigma_{\theta}+\frac{v}{E} \cdot\left(\sigma_{r}+\sigma_{z}\right) \\
& \varepsilon_{z}=\frac{1}{E} \cdot \sigma_{z}+\frac{v}{E} \cdot\left(\sigma_{\theta}+\sigma_{r}\right) . \\
& \varepsilon_{r z}=\left(\frac{1+v}{E}\right) \cdot \tau_{r z} \\
& \varepsilon_{z \theta}=\varepsilon_{r \theta}=0
\end{aligned}
$$

A scalar 'stress function' $\phi(r, z)$ that satisfies $\nabla^{4} \phi=0$ can be used to derive stresses and displacements (Love, 1923):

$$
\begin{aligned}
& \sigma_{r}=\frac{\partial}{\partial z}\left(v \cdot \nabla^{2} \phi-\frac{\partial^{2} \phi}{\partial r^{2}}\right) \\
& \sigma_{\theta}=\frac{\partial}{\partial z}\left(v \cdot \nabla^{2} \phi-\frac{1}{r} \cdot \frac{\partial \phi}{\partial r}\right) \\
& \sigma_{z}=\frac{\partial}{\partial z}\left((2-v) \cdot \nabla^{2} \phi-\frac{\partial^{2} \phi}{\partial z^{2}}\right) \\
& \tau_{r z}=\frac{\partial}{\partial r}\left((1-v) \cdot \nabla^{2} \phi-\frac{\partial^{2} \phi}{\partial z^{2}}\right) \\
& w=\frac{1+v}{E} \cdot\left((1-2 \cdot v) \cdot \nabla^{2} \phi+\frac{\partial^{2} \phi}{\partial r^{2}}+\frac{1}{r} \cdot \frac{\partial \phi}{\partial r}\right) \\
& u=-\frac{1+v}{E} \cdot\left(\frac{\partial^{2} \phi}{\partial r \partial z}\right)
\end{aligned}
$$

where $u$ and $w$ denote the $r$ and $z$ components of the displacement (respectively) and $\nabla^{2}$ is the Laplace operator $\nabla^{2}=\partial^{2} / \partial r^{2}+(1 / r) \cdot \partial / \partial r+\partial^{2} / \partial z^{2}$.

Consider a semi-infinite linear elastic isotropic and homogeneous medium made of $n-1$ parallel layers lying over a half-space. Each layer is identified by a subscript $i$ with material properties $E_{i}$ and $v_{i}$. The layers are numbered serially, with the layer at the top being layer 1 and the half-space, layer $n$. The origin of the cylindrical 
coordinate system is placed at the surface of the first layer with the $z$-axis drawn into the medium and the $r$-axis parallel to the layers. The depth to the individual interfaces, measured from the surface, is denoted by $z_{i}(i=1,2, . . n-1)$. Hence, $z_{1}$ is the thickness of layer $1, z_{2}$ is the combined thickness of layers 1 and 2, and so on. The combined thickness of the $n-1$ layers is denoted by $H$ (i.e., $H=z_{n-1}$ ).

Following Huang (2004), a ‘stress function' that complies with all of the above requirements is:

$$
\phi_{i}(\rho, \lambda)=\left(\frac{H^{3} \cdot J_{0}(m \cdot \rho)}{m^{2}}\right) \cdot\left(\begin{array}{l}
A_{i} \cdot e^{-m \cdot\left(\lambda_{i}-\lambda\right)}-B_{i} \cdot e^{-m \cdot\left(\lambda-\lambda_{i-1}\right)} \\
+C_{i} \cdot m \cdot \lambda \cdot e^{-m \cdot\left(\lambda_{i}-\lambda\right)}-D_{i} \cdot m \cdot \lambda \cdot e^{-m \cdot\left(\lambda-\lambda_{i-1}\right)}
\end{array}\right) \cdots
$$

in which $\rho=r / H, \lambda=z / H, \lambda_{i}=z_{i} / H$ and $m$ is a unitless parameter; $A_{i}, B_{i}, C_{i}$ and $D_{i}$ are all unitless functions of $m$; $J_{k}$ denotes a Bessel function of the first kind of order $k$; and the subscript $i$ refers to the layer number. Substitution of this equation into equations 4.2.2 yields the response of interest in a given layer $i$ due to a vertical non-dimensional surface load of the form $m \cdot J_{0}(m \cdot \rho)$. The value of the functions $A_{i}(m), B_{i}(m), C_{i}(m)$ and $D_{i}(m)$ cannot be expressed analytically; they must be determined, for any given value of $m$, by solving a set of linear equations. This set of equations transpires from the boundary and continuity conditions of the problem as follows:

$$
\begin{aligned}
& \left(\sigma_{z}^{*}\right)_{1}=m \cdot J_{0}(m \cdot \rho) \quad \text { for } \quad \lambda=0 \\
& \left(\tau_{r z}^{*}\right)_{1}=0 \quad \text { for } \quad \lambda=0 \\
& \left(\sigma_{z}^{*}\right)_{i}=\left(\sigma_{z}^{*}\right)_{i+1} \quad \text { for } \quad \lambda=\lambda_{i} \\
& \left(\tau_{r z}^{*}\right)_{i}=\left(\tau_{r z}^{*}\right)_{i+1} \quad \text { for } \quad \lambda=\lambda_{i} \\
& \left(w^{*}\right)_{i}=\left(w^{*}\right)_{i+1} \quad \text { for } \quad \lambda=\lambda_{i} \\
& \left(u^{*}\right)_{i}=\left(u^{*}\right)_{i+1} \quad \text { for } \quad \lambda=\lambda_{i} \\
& \left(R^{*}\right)_{n}=0 \quad \text { for } \quad \lambda \rightarrow \infty
\end{aligned}
$$


in which the asterisk is used to indicate that the response is due to vertical surface loading $m \cdot J_{0}(m \cdot \rho)$ as can also be seen in equations 4.2.4a-b. Equations 4.2.4c-f express the continuity of stresses and displacements inside the structure at the layer interfaces; full bonding is suggested by equation 4.2.4f. Equation 4.2.4g means that all response types (denoted using $R$ ) must vanish for the $n^{\text {th }}$ layer and at infinite depth (i.e., $\lim _{z \rightarrow \infty} R=0$ ). Finally, the response due to a uniform load $q$ distributed over a circular area of radius $\bar{a}$ is obtained by performing the integration:

$$
R=(q \cdot \alpha) \cdot \int_{m=0}^{\infty} \frac{R^{*}}{m} \cdot J_{1}(m \cdot \alpha) \cdot d m
$$

in which $\alpha=\bar{a} / H$ and $R$ is the stress or displacement of interest. Strains are thereafter obtained using the constitutive relations (i.e., equations 4.2.1a-d).

For the purpose of this study, the entire aforementioned derivation was programmed into an Excel worksheet (see program ELLEA1 in Appendix B). This was done for the case of five layers and considering two separate loaded areas. The combined effect of the two independent loads is calculated using superposition after converting the axially symmetric results in each case to a Cartesian coordinate system. The integration in equation 4.2.5 was carried out numerically between the first 200 zeros of the Bessel functions involved. The Gauss integration scheme was used for this purpose whereby the first interval was integrated using a 30-point Gaussian formula, the second interval was integrated using a 20-point formula, the third interval was integrated using a ten-point formula and the remaining intervals were integrated using a five-point formula. In order to speed the computational time, the number of matrix inversions required for solving equations 4.2.4 was limited to 96, corresponding to 96 predetermined values of the integration variable $m$ in the range of 0 to 50,000. A cubic spline interpolation scheme was used to derive intermediate results within this range. Furthermore, in order to improve the convergence of the integration, especially for points residing close to the surface, one step of Richardson extrapolation was employed (Sugihara, 1987). 
The program's user interface is shown in Figure 4.2.1. As can be seen (from top to bottom), the input of material properties and layer thicknesses is done in the topmost table. For each layer three attributes are required: Young's modulus, Poisson's ratio, and thickness. In the example shown in Figure 4.2.1 only four layers are considered because identical material properties are assigned to layers 3 and 4 (recall that all layers are fully bonded). Next, the required loading information is defined for each of the two loads, consisting of a vertical stress magnitude, loading radius, and the location of application. In the example shown the loaded areas differ in their stress magnitude (105 vs. 55) and radius (4 vs. 7), and their locations of application are specified by the $X$ and Y coordinates. Finally, the coordinates of the evaluation point within the structure are required. In the example they are $x=2, y=2$ and $z=2$; these values are relative to the selected loading coordinates.

\begin{tabular}{|c|c|c|c|c|c|}
\hline Layer & Modulus & Poisson & Thickness & & \\
\hline 1 & 300,000 & 0.30 & 5.0 & & \\
\hline 2 & 24,000 & 0.35 & 6.0 & & \\
\hline 3 & 12,000 & 0.40 & 19.0 & & \\
\hline 4 & 12,000 & 0.40 & 42.0 & & \\
\hline 5 & $4,300,000$ & 0.20 & semi-inf & & \\
\hline Load\# & Stress & Radius & $\mathrm{x}$ & $Y$ & \\
\hline 1 & 105.00 & 4.00 & -10.00 & 0.00 & \\
\hline 2 & 55.00 & 7.00 & 10.00 & 0.00 & \\
\hline & & & & & \\
\hline & $\mathrm{x}$ & $\mathbf{y}$ & z & & \\
\hline Evaluation Point & 2.00 & 2.00 & 2.00 & Layer= 1 & 1 \\
\hline & & & & & \\
\hline Direction & Stress & Strain (microstrains) & $1000 *$ Disp. & & \\
\hline $\mathrm{x}$ & 22.5 & 40 & & & \\
\hline $\mathbf{Y}$ & 27.1 & 60 & & & \\
\hline Z & 8.2 & -22 & 20.8 & Positive $=1$ & Down \\
\hline YZ & 7.7 & Negative (stress or s & train) $=$ Tensio & & \\
\hline$X Z$ & -20.2 & & & & \\
\hline$X Y$ & -1.0 & & & & \\
\hline
\end{tabular}

Figure 4.2.1: User interface of the isotropic LET program ELLEA1 (see Appendix B).

Based on the chosen depth for the evaluation point $z$, the program identifies automatically the layer in question (layer \#1 in the example). The resulting stresses, strains and vertical deflection are shown in the bottom table. It should be noted that 
there is no requirement to press a 'run' button to execute the code; in fact, any change of value in one of the input tables will be automatically reflected in real time in the results table. This feature is what makes this program extremely easy to use and appealing for further analyses compared to any other available LET code. Note also that no units are specified as the computations are done in dimensionless form (see Equation 4.2.3); the user must be consistent with his choice. In the example, units of psi are used for moduli and stresses; inches are used for thicknesses, radii and coordinates.

\subsubsection{Calibration to APT Conditions}

It is well recognized that pavement materials do not comply with isotropic LET assumptions. The resilient response of HMA mixtures is known to be anisotropic and nonlinear viscoelastic (Shields et al., 1998; Levenberg, 2006; Uzan and Levenberg, 2007). The resilient response of unbound layers is nonlinear elastic and stress-state sensitive (Uzan, 1985; 1992) and also anisotropic (e.g., Tutumluer and Thompson, 1997). As argued in Section 4.1 use of isotropic LET may be considered appropriate, at least as a first order approximation, given that as-constructed pavement layers are not stress-free even without external loads. These result in built-in stresses which diminish somewhat the inconsistency with actual material behavior. Nevertheless, a systematic error is introduced into the analysis when isotropic LET is applied. Minimizing this error can be accomplished by deriving the free model parameters (i.e., elastic moduli) through a process of inverse analysis (or backcalculation) using the time history of embedded gauge readings.

Following this approach, subsequent stresses, strains and deflections calculated with the calibrated model will resemble measured responses even though the model assumptions are fundamentally incorrect and over-simplified. In this connection it should be noted that LET cannot inherently simulate certain features that were seen in the experiment (see Subsection 3.5.2). One example refers to the offset observed between peak responses and load location which in LET must coincide. Another example is the non-symmetric response relative to the load location, i.e., the differences between approaching and receding curves as recorded by the gauges which in LET is always symmetric (see also Elseifi et al., 2006; Al-Qadi, 2007). 
For performing the backcalculation, the $\mathrm{n} 1$ pavement system was represented using four layers. The three HMA lifts were combined into one (top) layer, 5 in. (127 $\mathrm{mm}$ ) thick with an assumed Poisson's ratio of $v_{1}=0.30$. The second layer from the top represented the crushed aggregate base course, with a thickness of 6 in. (152.4 mm) and $v_{2}=0.35$ (assumed). Because no instrumentation was embedded in the subgrade (only on top), there was no available data to support its sub-layering. Hence, the upper and lower subgrade layers were combined into one layer (third layer from the top) having a total thickness of $61 \mathrm{in}$. $(1.55 \mathrm{~m})$ and $v_{3}=0.40$ (assumed). The fourth and final layer, with semi-infinite thickness, represented the concrete floor of the test pit. The elastic properties of this layer were fixed to the following values: $E_{4}=4,000,000$ psi $(27,580$ $\mathrm{MPa}$ ) and $v_{4}=0.20$. The dual-wheel loading was represented by two circular areas, each 8 in. (203 mm) in diameter, transferring uniform vertical stresses of 150 psi (1.03 $\mathrm{MPa}$ ) to the pavement surface. The spacing between the centers of the loads was taken as 13.5 in. (343 mm). For simulating the moving APT carriage, the quasi-static approach was applied in which dynamic (inertial) effects are disregarded. This assumption seemed reasonable because of the relatively slow loading speeds in the APT.

Generated model responses were compared to measured responses and a nonlinear optimization algorithm (Fylstra et al., 1998) was applied to manipulate the material properties until a best fit was achieved. This process was repeated twice to separately analyze the structure during pass \#5,000 and during pass \#80,000. Due to the non-symmetric strain response of the pavement, only data from the approaching branch were used for the comparison. Subsequently, 25 data points were pre-selected from each time history, corresponding to 25 different APT carriage positions relative to the gauge location with denser spacing closer to the gauge. These 'offset' distances ranged between 70 in. $(1.78 \mathrm{~m})$, for which readings were negligible, and zero, in which the APT carriage was exactly in line with the gauge along the Y-axis (see Figure 3.5.1).

Regardless of the number of data points used for the comparison between model and experiment there were only three moduli that needed to be backcalculated (for a given pass level), namely the HMA modulus $\left(E_{1}\right)$, the aggregate base modulus $\left(E_{2}\right)$, 
and the subgrade modulus $\left(E_{3}\right)$. In order to derive their numerical values, an objective (scalar) function describing the agreement between the model and test data was formulated. First, for each gauge separately, out of the total twelve gauges available, an error term was defined as follows:

$$
E R R_{g}=\frac{1}{N} \cdot \sqrt{\sum_{n=1}^{N}\left[R_{n}^{\mathrm{APT}}-R_{n}^{\mathrm{model}}\right]^{2}}
$$

in which $N$ is the number of data points used for the comparison for the $g^{\text {th }}$ gauge (i.e., $N=25$ ). $R^{\mathrm{APT}}$ represents the measured APT response of either stress or strain and $R^{\text {model }}$ is the corresponding isotropic LET response. Note that $E R R_{g}$ has the same units as $R^{\mathrm{APT}}$ (or equivalently $R^{\text {model }}$ ) and is always positive. Next, these individual errors were combined to formulate a unitless global error term, defined as follows:

$$
\text { Global_Error }=\frac{1}{G} \cdot \sum_{g=1}^{G}\left[\frac{E R R_{g}}{\min \left(E R R_{g}\right)}-1\right]
$$

where $G$ is the total number of gauges considered in the analysis (i.e., $G=12$ ), and $\min \left(E R R_{g}\right)$ represents the lowest achievable error between the model and the test data for the $g^{\text {th }}$ gauge. The numerical value of $\min \left(E R R_{g}\right)$ was obtained by employing an over-fitting technique; i.e., the layer moduli were first manipulated using the optimization algorithm in an effort to separately minimize each of the individual errors (equation 4.2.6).

Note that $\min \left(E R R_{g}\right)$ is always greater than zero; even if the model were perfect, all test data contain some random noise. However, the global error term can, in principal, equal zero. This situation occurs mathematically when all individual errors are minimal. Therefore, equation 4.2.7 serves as a weighted average of the individual errors, making sure that neither of the gauge readings is underweighted or overweighted in the backcalculation process compared to the others. In order to enable a direct comparison between the global error for pass \#5,000 and pass \#80,000, values of 
$\min \left(E R R_{g}\right)$ obtained for pass \#5,000 were also used for the backcalculation of pass \#80,000.

\subsubsection{Interim Results and Discussion}

Table 4.2.1 presents the backcalculated layer moduli for pass \#5,000 and pass \#80,000. The global error term (equation 4.2.7) was $4.89 \%$ for pass \#5,000 and $6.27 \%$ for pass $\# 80,000$. In both cases it can be seen that the stiffness of the pavement structure is decreasing from top to bottom. During pass \#5,000 the HMA is 14.6 times stiffer than the underlying aggregate base. The aggregate base is seen to be twice as stiff as the subgrade. By comparing these results with pass \#80,000, it is clear that during the APT experiment the individual layer moduli increased: (i) the HMA experienced a slight stiffness increase of about 8.5\%; (ii) the stiffness of the base increased significantly by about 54\%; and (iii) the subgrade increased in stiffness by about 16.5\%. Subsequently, the relative stiffness within the structure also changed, with the HMA ending up 10.3 times stiffer than the underlying base, and the base becoming 2.6 times stiffer than the subgrade. In lieu of direct test data, these changes are believed to be the result of further densification under the APT carriage passes, especially of the unbound materials.

Table 4.2.1: Backcalculated layer moduli for pass \#5,000 and pass \#80,000.

\begin{tabular}{|c|c|c|c|c|c|}
\hline \multirow{2}{*}{ \# } & \multirow{2}{*}{ Layer } & \multirow{2}{*}{$\begin{array}{l}\text { Thickness, } \\
\text { in. (mm) }\end{array}$} & \multirow{2}{*}{$\begin{array}{c}\text { Poisson's } \\
\text { Ratio }\end{array}$} & Pass \#5,000 & Pass $\# \mathbf{8 0 , 0 0 0}$ \\
\hline & & & & \multicolumn{2}{|c|}{ Backcalculated Moduli, psi (MPa) } \\
\hline 1 & HMA & $5(127)$ & 0.30 & $350,000(2,412)$ & $380,000(2,618)$ \\
\hline 2 & Base & $6(152)$ & 0.35 & $24,000(165)$ & $37,000(255)$ \\
\hline 3 & Subgrade & $61(1,549)$ & 0.40 & $12,000(83)$ & $14,000(96)$ \\
\hline 4 & Concrete & $\begin{array}{l}\text { Semi- } \\
\text { infinite }\end{array}$ & 0.20 & \multicolumn{2}{|c|}{$4,000,000(27,580)$} \\
\hline
\end{tabular}

Figures 4.2.2 and 4.2.3 show both the measured and calibrated model responses for pass \#5,000 and \#80,000 (respectively) vs. offset distance from the gauge. Each figure contains six charts. The two topmost charts show horizontal strains in $\mathrm{X}$ (left) 
and in Y (right) for gauges located along the loading centerline. The charts in the middle of the figure show horizontal strains in $\mathrm{X}$ (left) and in $\mathrm{Y}$ (right) for gauges positioned outside the loading path. The bottom charts show vertical stresses as measured by pressure cells located on top of the base (left) and on top of the subgrade (right). In each chart the measured gauge data is represented by solid markers. Because the pavement was instrumented with pairs of gauges measuring the same response, two types of markers are used. The calibrated model responses are shown using a solid line.

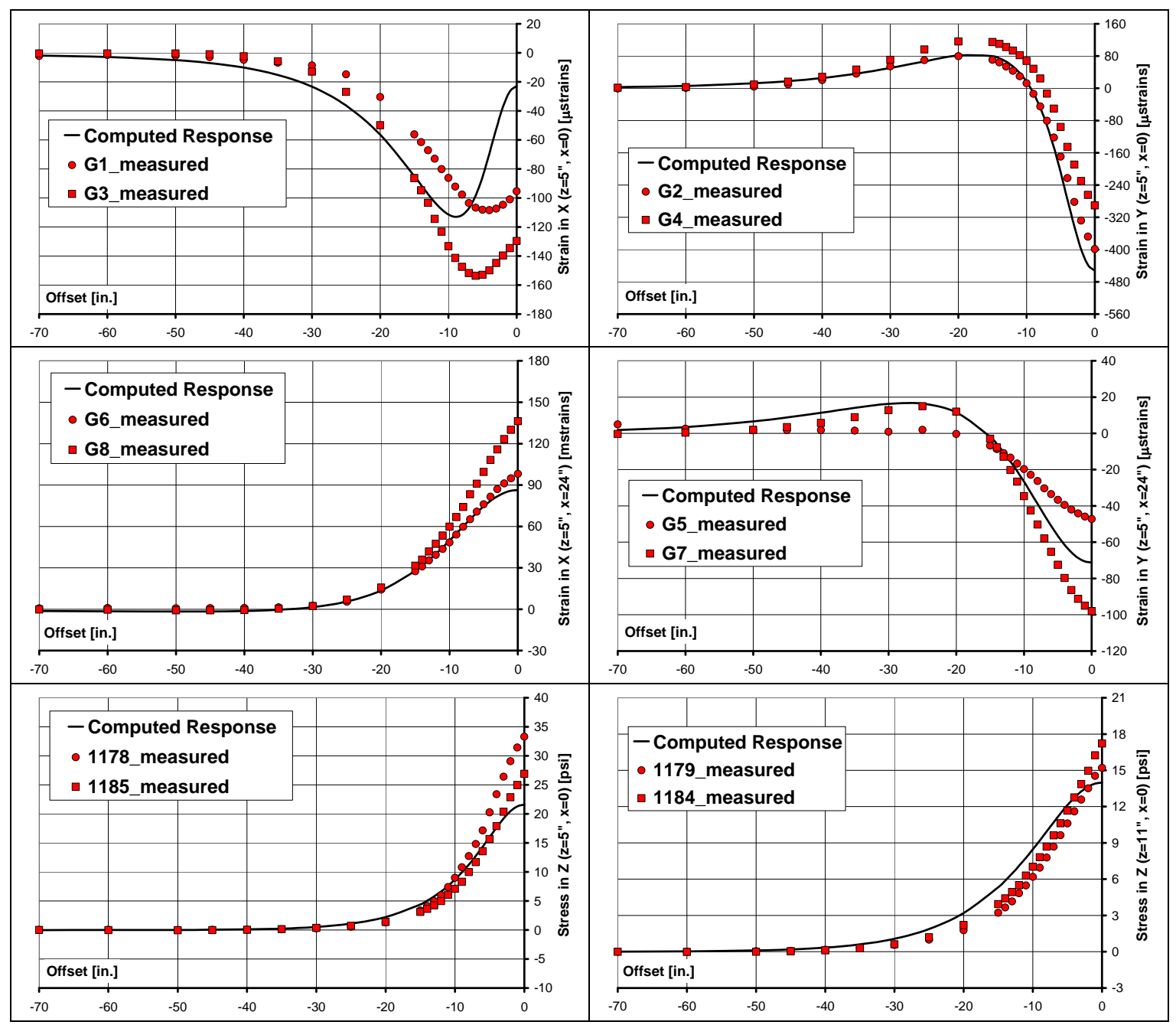

Figure 4.2.2: Resilient responses during APT pass \#5,000. Both measured (solid markers) and model generated (solid line) are shown.

These figures provide some intuition and information on several experimental and modeling aspects. First, the large difference between measured responses of the gauge pairs is demonstrated. Graphically, these differences seem to be smaller for the 
stress measurements compared to the strain readings. During pass \#5,000 the maximum relative difference in the peak stress readings with respect to the average reading at the peak is $10.6 \%$ (for pressure gauges 1178 and 1185). Similarly, the maximum relative difference in the peak strain readings with respect to their average at the peak is $35.0 \%$ (for strain gauges G5 and G7). During pass \#80,000 the corresponding differences are 10.8\% (again, pressure gauges 1178 and 1185) and 44.7\% (strain gauges G6 and G8). These differences are believed to represent both structural heterogeneity (see also Figures 3.5.6 to 3.5.18) and slight dissimilarity in gauge installation conditions.

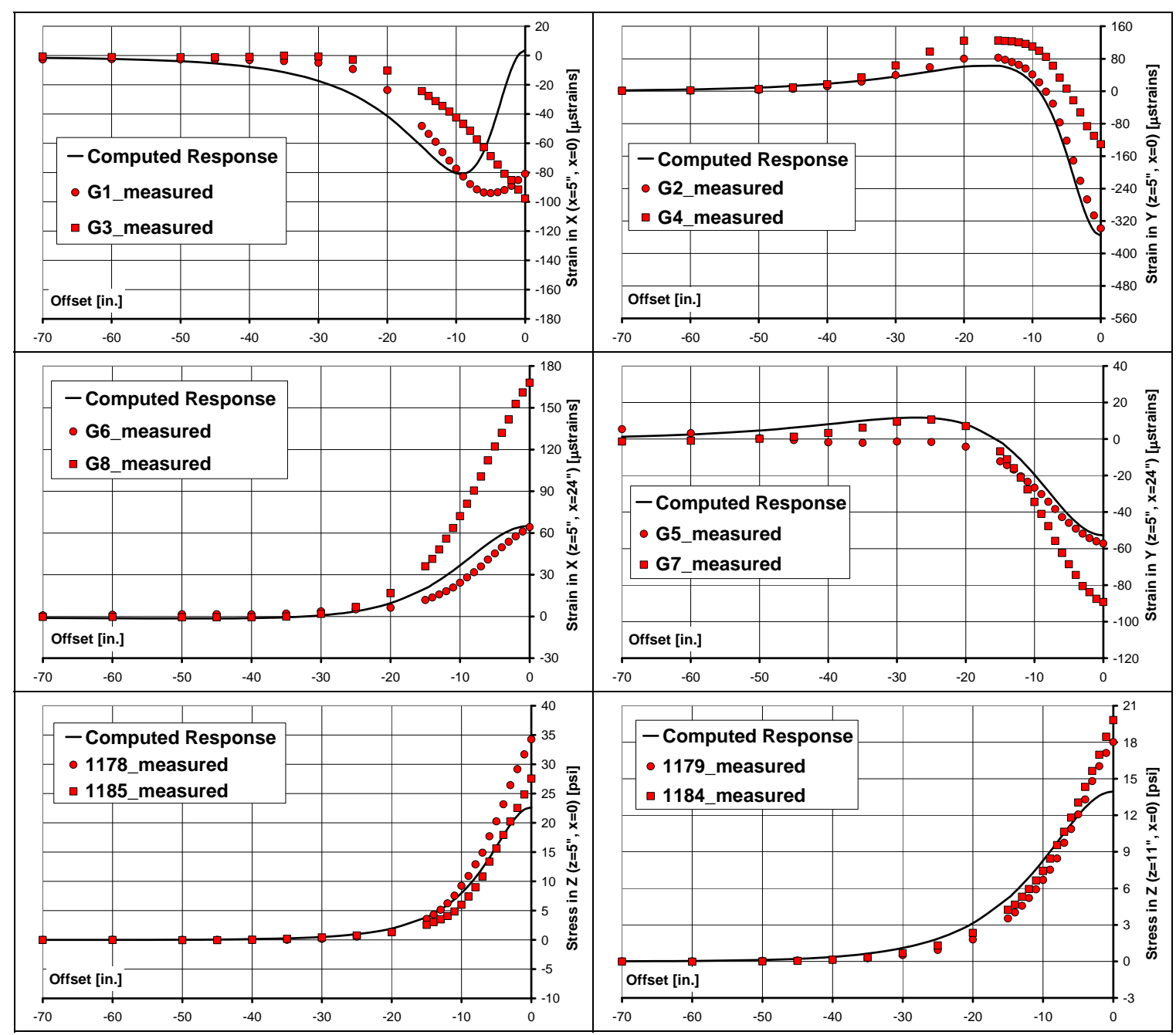

Figure 4.2.3: Resilient responses during APT pass \#80,000. Both measured (solid markers) and model generated (solid line) are shown.

Next, the goodness of fit of the calibrated model can be visualized. It may be graphically seen that the isotropic LET captures relatively well the horizontal strains in 
$\mathrm{X}$ and $\mathrm{Y}$ directions for the off-center gauges (G5 to G8). For the centerline gauges (G1 to G4), the horizontal strains are captured relatively well only in the direction of loading (G2 and G4). The fit is not very good for the strains in the transverse direction (G1 and G3). The vertical stress peaks on top of the base (1178 and 1185) and on top of the subgrade (1179 and 1184) are underpredicted by the model. The above findings, however, should not be expected to hold in general. In other cases, the stress dependence of the unbound layers, and perhaps even anisotropy, may impair the theory's reproducibility. Also, the ability to successfully use LET is likely to weaken when the pavement structure is comprised of thicker HMA layers. In this case, the HMA's time dependence will be more dominant and the non-symmetry in the strain and stress response within the structure will be more pronounced.

\subsection{NCAT RESPONSE PREDICTION}

\subsubsection{Methodology}

The calibrated APT model (Table 4.2.1) cannot be used directly to forecast NCAT responses. The main differences that need to be taken into account are HMA temperature, axle configuration, axle weight and loading speed. It is straightforward to apply the layered model with different axle configurations and different axle loads. Because the quasi-static approach is applied for simulating the moving load, speed is not an issue from a computational standpoint. However, the HMA properties themselves are sensitive to the loading speed and temperature. This can be accounted for exogenously by changing the HMA modulus in the NCAT simulation; the methodology is described hereafter.

As a first step, the complex modulus test data from the individual HMA mixes are combined into one dataset representative of one HMA layer that is 5 in. (127 mm) thick. The following equations are suggested for this purpose:

$$
E_{1}^{\text {com }}=\left[\frac{h_{1} \cdot \sqrt[3]{E_{1,1}}+h_{2} \cdot \sqrt[3]{E_{1,2}}}{h_{1}+h_{2}}\right]^{3}
$$




$$
\begin{aligned}
& E_{2}^{\text {com }}=\left[\frac{h_{1} \cdot \sqrt[3]{E_{2,1}}+h_{2} \cdot \sqrt[3]{E_{2,2}}}{h_{1}+h_{2}}\right]^{3} \\
& \left|E_{\text {com }}^{*}\right|=\sqrt{\left(E_{1}^{\text {com }}\right)^{2}+\left(E_{2}^{\text {com }}\right)^{2}} \\
& \phi_{\text {com }}=\arctan \left(\frac{E_{2}^{\text {com }}}{E_{1}^{c o m}}\right)
\end{aligned}
$$

in which $h_{1}=1.0$ in. $(25.4 \mathrm{~mm})$ is the lift thickness of Mix $1, h_{2}=4.0$ in. $(102 \mathrm{~mm})$ is the lift thickness of Mix 2 (refer to Figure 3.2.1), $E_{1, i}$ and $E_{2, i}(i=1,2)$ are the components of the complex modulus for Mix $i$ at a given test temperature and frequency (refer to Table 2.3.5, Table 2.3.6 and equation 3.3.3), and $\left|E_{\text {com }}^{*}\right|$ and $\phi_{c o m}$ are the combined dynamic modulus and phase angle at a given test temperature and frequency.

The results from these computations are shown in Table 4.3.1 from which master curves were constructed for a reference temperature of $15.5^{\circ} \mathrm{C}\left(60^{\circ} \mathrm{F}\right)$ using the approach developed by Levenberg and Shah (2008). Referring to equations 3.3.4, 3.3.5, 3.3.6 and 3.3.7 (Subsection 3.3.2), the derived parameters for the combined properties were: $a_{1}=2,328 \mathrm{MPa}(337.6 \mathrm{ksi}), a_{2}=1.03 \cdot 10^{-2}, a_{3}=6.889 \cdot 10^{-5} \mathrm{~s}, E_{\infty}=164 \mathrm{MPa}$ (23.8 ksi), $c_{1}=33.0$, and $c_{2}=302.1^{\circ} \mathrm{C}\left(575.8^{\circ} \mathrm{F}\right)$. The resulting dynamic modulus and phase angle master curves (vs. reduced frequency $f_{r}$ ) are plotted in Figure 4.3.1. The corresponding time-temperature shift factor $\left(a_{T}\right)$ vs. physical temperature is plotted in Figure 4.3.2.

Next step, referring to Table 4.2.1, recall that the backcalculated modulus of the HMA layers was 350,000 psi (2,412 MPa) in the initial part of the experiment. This value is suitable for a temperature of $15.5^{\circ} \mathrm{C}\left(60^{\circ} \mathrm{F}\right)$ and a loading speed of $5 \mathrm{mph}(\sim 2.2$ $\mathrm{m} / \mathrm{s}$ ). Using Figures 4.3.1 and 4.3.2, it may be seen that this stiffness level is paired with a reduced frequency of $0.0232 \mathrm{~Hz}$ and a time-temperature shift factor of 1.0. Using the same figures a new HMA modulus can be computed for any given loading speed and temperature by adjusting $f_{r}$ and $a_{T}$ relative to the APT conditions. For example, at 
NCAT the trucks are traveling at $45 \mathrm{mph}(20.1 \mathrm{~m} / \mathrm{s})$; this speed is $9.0(=45 / 5)$ times higher than in the APT. Now, if the temperature was $15.5^{\circ} \mathrm{C}\left(60^{\circ} \mathrm{F}\right)$ then $a_{T}=1.0$ and the reduced frequency would become $0.2088 \mathrm{~Hz}(=0.0232 \cdot 9.0 \cdot 1.0)$; the corresponding modulus (using Figure 4.3.1) is therefore 4,028 MPa (584,100 psi). If, on the other hand, the HMA temperature at NCAT was $30.0^{\circ} \mathrm{C}\left(86^{\circ} \mathrm{F}\right)$ instead of $15.5^{\circ} \mathrm{C}\left(60^{\circ} \mathrm{F}\right)$, then $a_{T}=0.0308$ and the reduced frequency would become $0.0064 \mathrm{~Hz}$ $(=0.0232 \cdot 9.0 \cdot 0.0308)$; the corresponding modulus in this case is $1,685 \mathrm{MPa}(244,300$ psi).

In summary, using the combined dynamic modulus master curve with the newly computed (reduced) frequency, one can adjust the HMA modulus to adequately represent different conditions. This methodology is applied in the next subsections to forward calculate responses of interest at NCAT. Thereafter, the forecast is compared with measured values to assess the scheme.

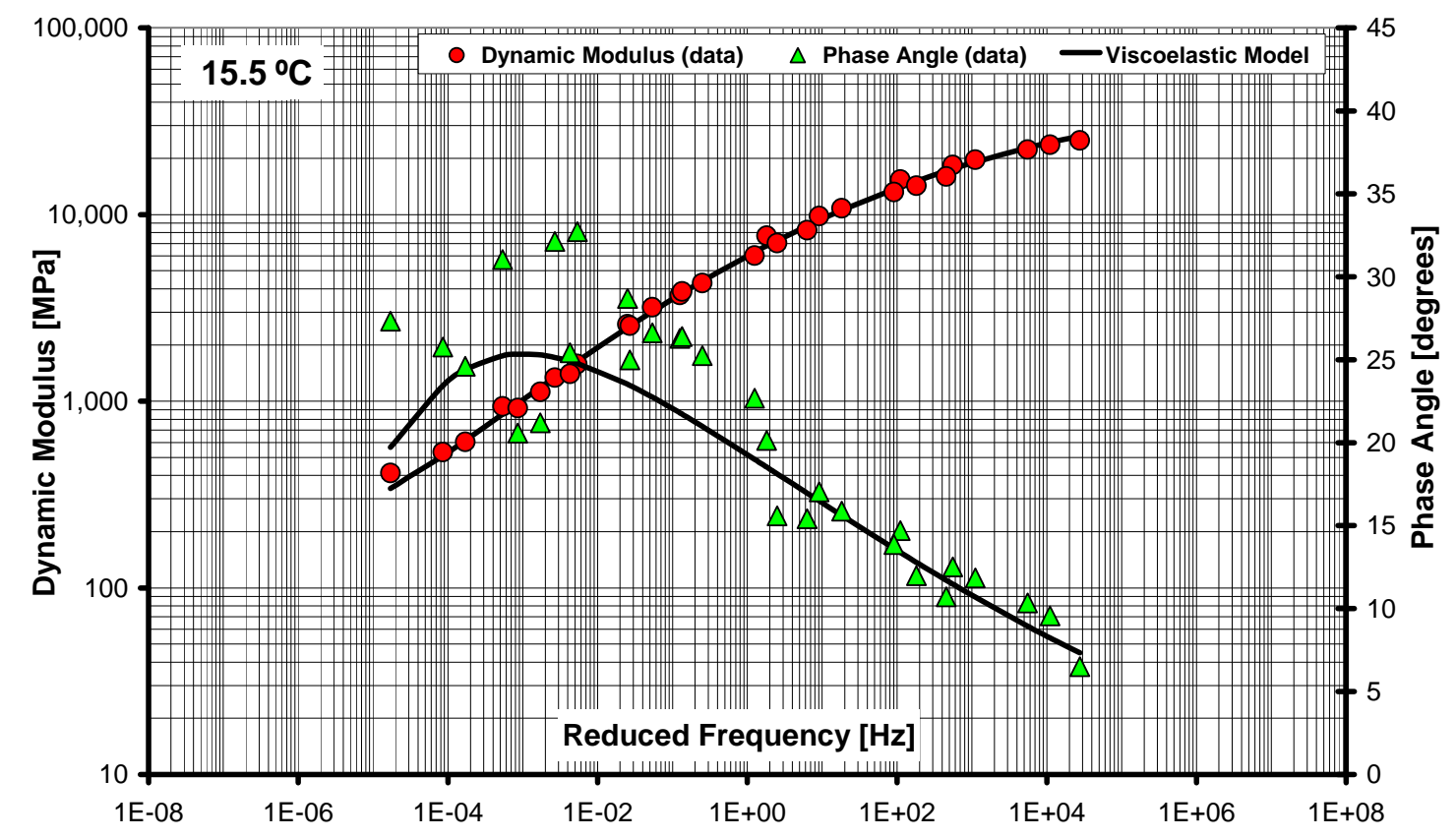

Figure 4.3.1: Combined HMA dynamic modulus and phase angle master curves for a reference temperature of $15.5^{\circ} \mathrm{C}$ (based on Table 4.3.1). 
Table 4.3.1: Combined complex modulus properties for APT n1 / NCAT N1 (based on equations 4.3 .1 to 4.3 .4 ).

\begin{tabular}{|c|c|c|c|}
\hline $\begin{array}{l}\text { Test Temperature } \\
{ }^{\circ} \mathrm{C}\left({ }^{\circ} \mathrm{F}\right)\end{array}$ & $\begin{array}{c}\text { Test Frequency } \\
{[\mathrm{Hz}]}\end{array}$ & $\begin{array}{c}\text { Dynamic Modulus } \\
\text { MPa (ksi) }\end{array}$ & $\begin{array}{c}\text { Phase Angle } \\
\text { [degrees] }\end{array}$ \\
\hline \multirow{6}{*}{$\begin{array}{l}-10.0 \\
(+14)\end{array}$} & 25 & 24,951 (3619) & 6.5 \\
\hline & 10 & 23,669 (3433) & 9.6 \\
\hline & 5 & 22,329 (3239) & 10.3 \\
\hline & 1 & $19,653(2850)$ & 11.8 \\
\hline & 0.5 & $18,426(2672)$ & 12.5 \\
\hline & 0.1 & 15,427 (2237) & 14.7 \\
\hline \multirow{6}{*}{$\begin{array}{c}+4.4 \\
(+40)\end{array}$} & 25 & 15,995 (2320) & 10.7 \\
\hline & 10 & $14,299(2074)$ & 12.0 \\
\hline & 5 & 13,229 (1919) & 13.9 \\
\hline & 1 & $10,814(1568)$ & 15.9 \\
\hline & 0.5 & $9,832(1426)$ & 17.0 \\
\hline & 0.1 & 7,693 (1116) & 20.1 \\
\hline \multirow{6}{*}{$\begin{array}{l}+21.1 \\
(+70)\end{array}$} & 25 & 8,265 (1199) & 15.4 \\
\hline & 10 & 7,038 (1021) & 15.6 \\
\hline & 5 & $6,037(876)$ & 22.7 \\
\hline & 1 & $4,295(623)$ & 25.2 \\
\hline & 0.5 & 3,708 (538) & 26.3 \\
\hline & 0.1 & 2,580 (374) & 28.7 \\
\hline \multirow{6}{*}{$\begin{array}{c}+37.8 \\
(+100)\end{array}$} & 25 & $3,873(562)$ & 26.4 \\
\hline & 10 & $3,188(462)$ & 26.6 \\
\hline & 5 & 2,543 (369) & 25.0 \\
\hline & 1 & $1,584(230)$ & 32.7 \\
\hline & 0.5 & 1,339 (194) & 32.1 \\
\hline & 0.1 & 939 (136) & 31.0 \\
\hline \multirow{6}{*}{$\begin{array}{c}+54.4 \\
(+130)\end{array}$} & 25 & $1,405(204)$ & 25.4 \\
\hline & 10 & 1,122 (163) & 21.2 \\
\hline & 5 & 918 (133) & 20.6 \\
\hline & 1 & $606(88)$ & 24.6 \\
\hline & 0.5 & $534(77)$ & 25.8 \\
\hline & 0.1 & $413(60)$ & 27.3 \\
\hline
\end{tabular}




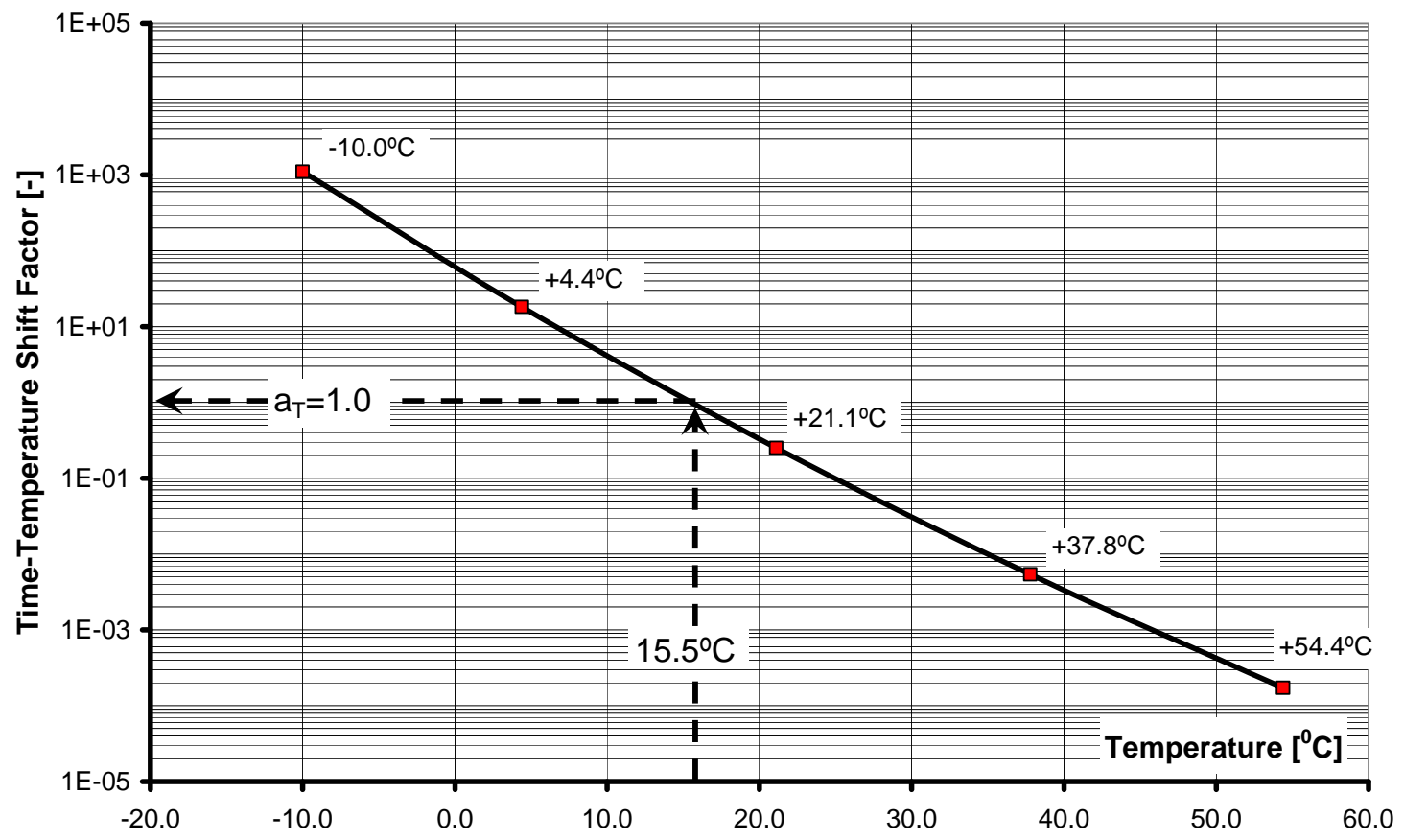

Figure 4.3.2: Combined HMA time-temperature shifting for a reference temperature of $15.5^{\circ} \mathrm{C}$ (based on Table 4.3.1).

\subsubsection{Falling Weight Deflections}

In this subsection an attempt is made to use the layered model, calibrated against APT data (Subsection 4.2.2) and adjusted using laboratory tests (Subsection 4.3.1), to forecast peak FWD deflections measured at NCAT (see Table 2.3.9). Given that the diameter of the FWD plate is $11.8 \mathrm{in}$. (300 mm) and assuming a load pulse of 0.04 seconds in duration, the 'speed' of the loading in the FWD test is 295 in./s (= 11.8/0.04). This is 3.35 times faster than the APT speed (= 295/88). Referring to NCAT section N1 before it incurred significant damage, the first three deflection tests in Table 2.3.9 are considered. The corresponding HMA temperatures were (respectively): $32.9^{\circ} \mathrm{C}$ $\left(91.2^{\circ} \mathrm{F}\right)$ on November $3,2003,16.7^{\circ} \mathrm{C}\left(62.1^{\circ} \mathrm{F}\right)$ on December 15,2003 , and $10.1^{\circ} \mathrm{C}$ $\left(50.2^{\circ} \mathrm{F}\right)$ on January 26,2004 . The appropriate time-temperature shift factors from Figure 4.3.2 are therefore: $\left.a_{T}\right|_{\text {Nov. } 03}=0.016,\left.a_{T}\right|_{\text {Dec. } 03}=0.704$ and $\left.a_{T}\right|_{\text {Jan.04 }}=3.987$. Using these values the adjusted reduced frequencies become:

$$
\left.f_{r}\right|_{\text {Nov. } 03}=0.0232 \cdot 3.35 \cdot 0.016=0.001 \mathrm{~Hz}
$$




$$
\begin{aligned}
& \left.f_{r}\right|_{\text {Dec } .03}=0.0232 \cdot 3.35 \cdot 0.704=0.055 \mathrm{~Hz} \\
& \left.f_{r}\right|_{\text {Jan. } 04}=0.0232 \cdot 3.35 \cdot 3.987=0.310 \mathrm{~Hz}
\end{aligned}
$$

in which 0.0232 is the reduced frequency derived from inverse analysis of APT conditions (see Subsection 4.3.1). Using Figure 4.3.1 the corresponding HMA moduli are: $\left.E_{\text {нмА }}\right|_{\text {Nov. } 03}=1,000 \mathrm{MPa}(145,000 \mathrm{psi}),\left.E_{\text {HмA }}\right|_{\text {Dec. } 03}=2,900 \mathrm{MPa}(420,500 \mathrm{psi})$ and $\left.E_{\text {HмA }}\right|_{\text {Jan. } 04}=4,600 \mathrm{MPa}(667,000 \mathrm{psi})$.

Figure 4.3.3 shows the peak measured FWD deflections at NCAT and also the corresponding computed deflections. The latter are based on the isotropic LET with fixed moduli for the base and subgrade, but with different HMA moduli based on the discussion above.

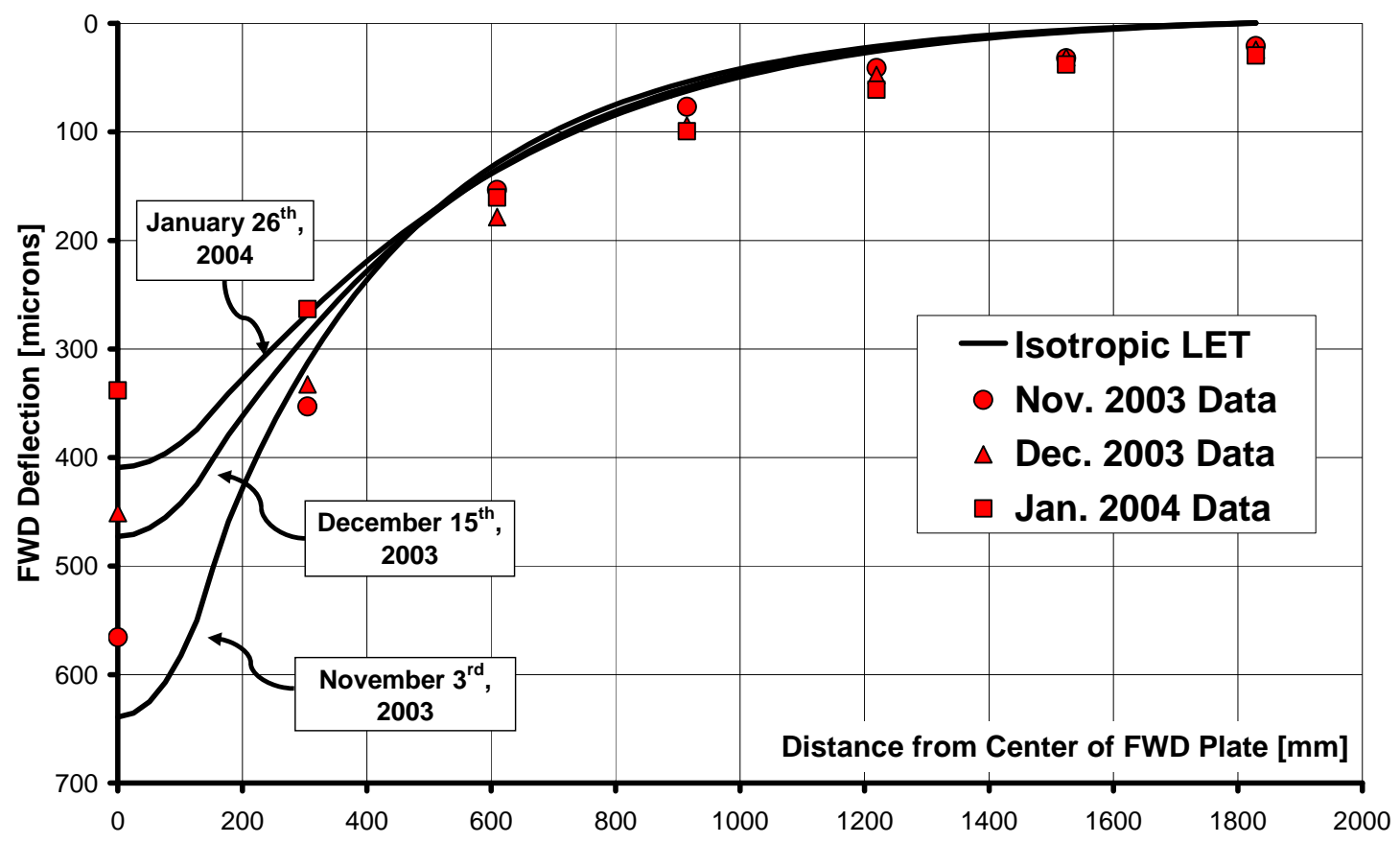

Figure 4.3.3: Measured and projected peak FWD deflections at NCAT N1.

The agreement between the test data and model projections can be graphically evaluated from the figure. It may be seen that the trend of the computations matches the trend in the deflection data. Quantitatively, the average absolute difference between test and model for the three data sets is 32.4 microns; the average absolute relative error is 
44.1\%. If the isotropic LET model was directly calibrated using the NCAT deflections, allowing only for the HMA modulus to differ in each case, the errors would have been 12.8 microns and $22.2 \%$ respectively. Therefore, although the trend in the computations follows the trend in the data, the forecasting errors are 2.5 to 2.0 times higher compared to the calibrated case.

\subsubsection{Traffic Induced Stresses and Strains}

In this subsection an attempt is made to forecast resilient stresses and strains at NCAT caused by one truck pass. As indicated in Section 4.1 the analysis is focused on the initial phase of the experiment before the HMA had incurred any visible fatigue cracking. Assuming no interaction between the different truck axles (see Table 2.1.1), the separate responses due to each half-axle are computed using isotropic LET. Model predictions are then compared with the responses shown in Figures 2.5.1 to 2.5.4 as measured by the gauge array in Figure 2.4.3.

First, the average temperature in the HMA is determined so that the timetemperature shift factor can be obtained from Figure 4.3.2. Using the three topmost data points in Table 2.5.1, the temperature profile in the HMA is expressed as a second degree polynomial: $T(z)=0.475 \cdot z^{2}-4.75 \cdot z+88.5$ in which $z$ represents depth from the surface in inches and $T$ has units of ${ }^{\circ} \mathrm{F}$. By substitution of the appropriate depths it can be verified that the expression reproduces the temperatures in Table 2.5.1. The average HMA temperature is thereafter calculated by integrating $T(z)$ with respect to $z$ over the total HMA thickness, between 0 and 5 in. (0 to $127 \mathrm{~mm}$ ), and dividing the outcome by 5 . Consequently, the average HMA temperature was found to be $80.6^{\circ} \mathrm{F}$ or $27.0^{\circ} \mathrm{C}$ which pairs with $a_{T}=0.062$ in Figure 4.3.2. Next, the HMA modulus is determined from Figure 4.3.1; the reduced frequency is simply calculated as follows: $f_{r}=0.0232 \cdot 9.0 \cdot 0.062=0.013 \mathrm{~Hz}$ in which $0.0232 \mathrm{~Hz}$ is the reduced frequency that represents the APT loading speed and temperature (Subsection 4.3.1), 9.0 accounts for the difference in loading speed between APT and NCAT (Subsection 4.3.1) and 0.062 is the time-temperature shifting (above). The resulting HMA modulus (see Figure 4.3.1) is 2,000 MPa (290,000 psi). All other layer properties are given in Table 4.2.1. 
In the forward computations, the radius of contact area for each of the tires was always taken as 4.0 in. (101.6 mm). The corresponding stress intensity was calculated using the axle weights in Table 2.1.1. For the dual axles, center to center tire spacing was taken as $13.5 \mathrm{in}$. (343 mm); for the dual tandem axles, axle spacing was taken as 50 in. $(1.27 \mathrm{~m})$. The moving NCAT truck was simulated by applying the array of tire loads at different locations relative to the gauges. Calculations were performed for every 0.001 seconds during which the axles traveled forward 0.792 in. $(20.1 \mathrm{~mm})$ based on a $45 \mathrm{mph}$ (792 in./s) speed. It is important to recall (see Subsection 2.5.1) that the wheel positions relative to the gauges were not measured in the NCAT experiment. These positions are necessary for performing the LET computations. In an effort to resolve this issue it is assumed hereafter that peak gauge readings were attained when the load was in line with the corresponding gauge along the $\mathrm{Y}$-axis in Figure 2.4.3. This assumption helps position the moving axles in the longitudinal direction (i.e., direction of travel). It is further assumed that the truck wheels were moving in a straight line, not necessarily parallel to road centerline while passing over the gauge array.

In Figure 4.3.4 the gauge array layout from Figure 2.4.3 is reproduced, showing only the functioning gauges that survived the construction process. Also shown in the figure, using connecting arrows, is the travel path of the center of the rightmost truck tire. This line is located at an unknown transverse distance from the BBC gauge, denoted in the figure as $X_{0}$ and an unknown distance from the ASC gauge, denoted as $X_{1}$. The determination of $X_{0}$ and $X_{1}$ is subsequently done, separately for each halfaxle considered, such that model predictions best conform with measured responses of these two gauges. Hence, the matching between model and experiment for gauges BBC and ASC should not be considered as pure prediction given that it was consciously minimized to position the axles. 


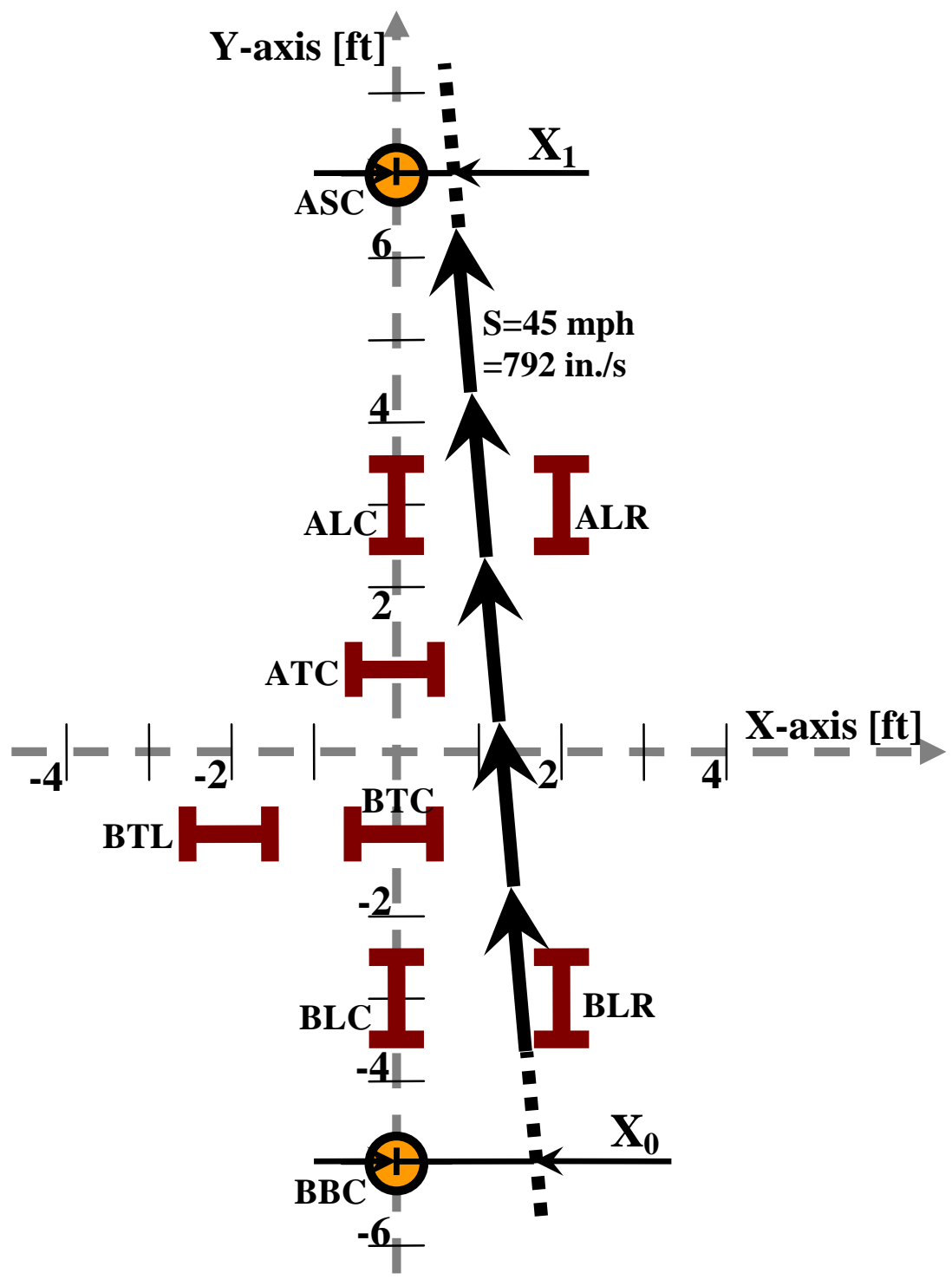

Figure 4.3.4: Layout of N1 gauge array (refer to Figure 2.4.3) and travel path positioning of the center point of the rightmost truck tire (connecting arrows).

The resulting numerical values of $X_{0}$ and $X_{1}$ are shown in Figure 4.3.5 for the different axles. Note that in this figure (and unlike Figure 4.3.4), the horizontal and vertical scales are different with the horizontal scale stretched to better illustrate the findings. The actual axle travel paths are not expected to be identical given that the 'train' of trailers has flexibility to move and 'worm around' in the transverse direction. Accordingly, as can be seen in the figure, the computed travel paths are similar but not identical, lying within a few inches from each other. Also noteworthy is the tendency of 
the axles following the drive axle to drift to the right side relative to the Y-axis. This is realistic considering the fact that the NCAT trucks traversed the N1 Section after completing a left turn on the East curve (see Figure 2.1.1).

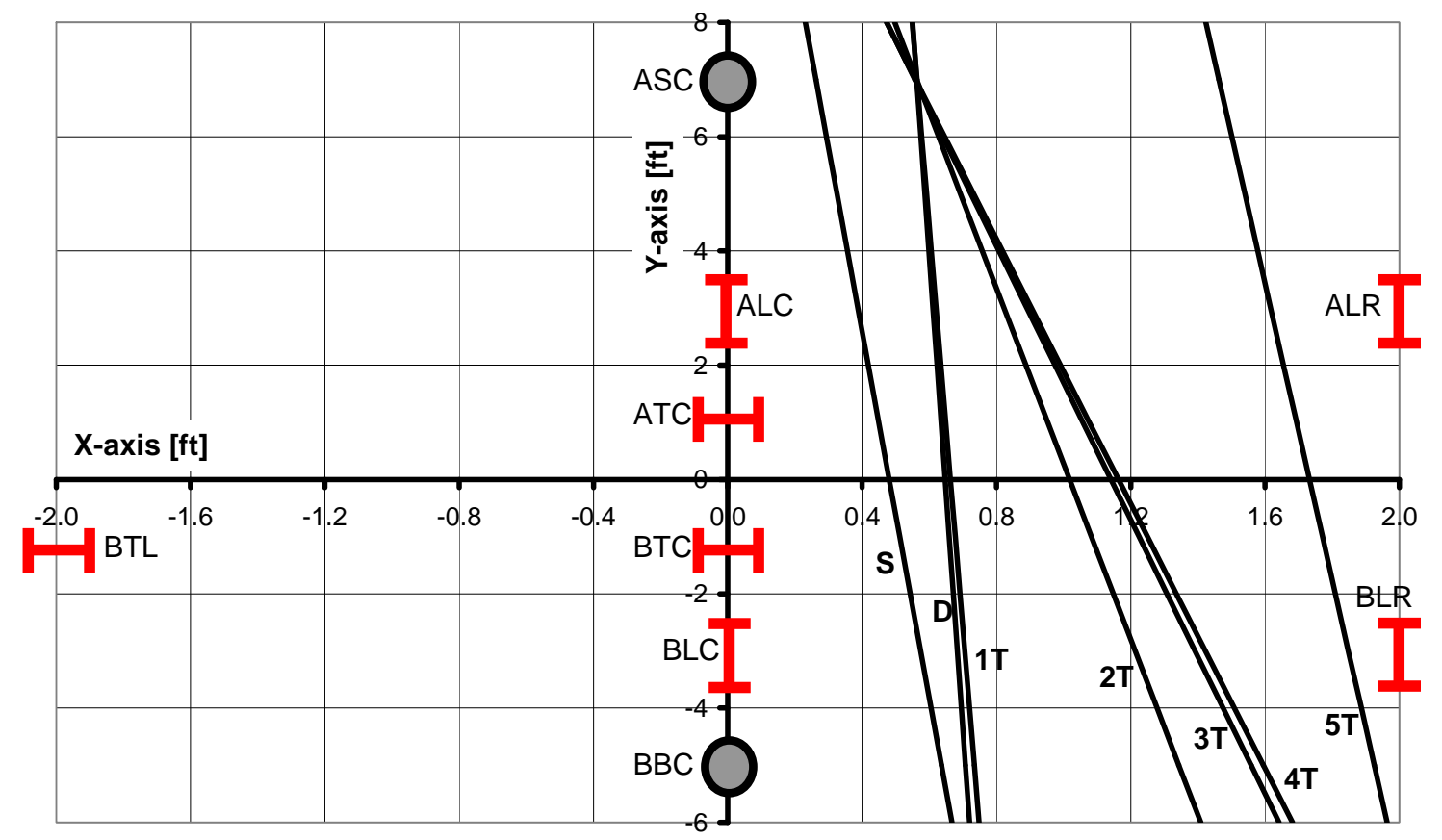

Figure 4.3.5: Travel paths of center of rightmost truck wheels over the N1 gauge array at NCAT for the different axles in Table 2.1.1.

The following Figures 4.3.6 to 4.3.10 graphically contrast the computational model and the measured resilient responses at NCAT (vs. time). Each figure separately presents the stresses and strains due to a different half-axle. Referring to Table 2.1.1, these are respectively: steering wheel (1S), drive axle (1D and 2D), first trailer axle (1T), third trailer axle (3T), and last (fifth) trailer axle (5T). Each figure is comprised of nine charts, depicting the calculated and measured response of the individual gauges shown in Figure 4.3.5. The abscissa represents time in seconds, matching the timeline in Figures 2.5.1 to 2.5.4. The ordinate depicts either vertical stress (in psi) or horizontal strain (in microstrains) depending on the gauge considered (note that the scale changes from case to case). In addition, each figure also includes a picture of the NCAT truck with an arrow identifying the half-axle considered.

As a general observation, these figures show that the model predictions capture relatively well the magnitudes as well as the trends in the measured responses. Similar 
to the APT case (refer to Subsection 4.2.3), better matching is usually achieved for the strains in the travel (longitudinal) direction (i.e., strains in Y) compared to the strains in the transverse direction (i.e., strains in $\mathrm{X}$ ). The forecastability of the vertical stresses cannot be assessed because these were used to allocate the loads.

Quantitatively, the matching errors in Figures 4.3.6 to 4.3.10, between the isotropic LET predictions and NCAT measured responses, are summarized in Table 4.3.2. These errors were computed using an expression similar to equation 4.2.6 using about 200 points of comparison spanning the timeframe shown in each of the charts. The reported error values should have all units of microstrains. However, because the strain magnitudes in the charts were different in each case a low error level would not necessarily mean better match. For this reason the individual errors were further normalized by the peak to peak magnitude of the corresponding measured response; the latter are shown in the table inside the brackets (units of microstrains). Subsequently, the resulting errors in the table are dimensionless (reported in percent), and as can be seen, range between $0.205 \%$ and $2.540 \%$. The lowest error refers to matching the response of the ALC gauge due to the fifth trailer axle (5T) - see Figure 4.3.10. The highest error refers to mismatching the response of the ATC gauge due to the first trailer axle (1T) - see Figure 4.3.8.

Table 4.3.2: Matching errors between isotropic LET predictions and NCAT measured responses. Errors are in percent after normalization using the corresponding peak to peak response shown in brackets (in microstrains).

\begin{tabular}{|l|c|c|c|c|c|}
\hline \multirow{2}{*}{ Gauge } & \multicolumn{5}{|c|}{ NCAT truck axle designation from Table 2.1.1 } \\
\cline { 2 - 6 } & $\mathbf{1 S}$ & 1D+2D & $\mathbf{1 T}$ & $3 \mathbf{3}$ & $\mathbf{5 T}$ \\
\hline BLC & $0.251(220)$ & $0.307(450)$ & $0.541(410)$ & $0.538(370)$ & $0.250(210)$ \\
\hline BLR & $0.346(100)$ & $0.653(260)$ & $1.030(200)$ & $0.656(360)$ & $0.357(510)$ \\
\hline BTC & $0.553(80)$ & $1.336(360)$ & $2.377(300)$ & $1.774(220)$ & $1.030(115)$ \\
\hline BTL & $0.540(40)$ & $0.863(100)$ & $1.220(100)$ & $1.376(90)$ & $0.858(80)$ \\
\hline ATC & $0.726(90)$ & $1.411(350)$ & $2.540(280)$ & $1.936(200)$ & $0.899(150)$ \\
\hline ALC & $0.371(200)$ & $0.270(500)$ & $0.438(450)$ & $0.668(320)$ & $0.205(220)$ \\
\hline ALR & $0.498(80)$ & $0.659(200)$ & $1.033(180)$ & $0.929(280)$ & $0.282(500)$ \\
\hline
\end{tabular}




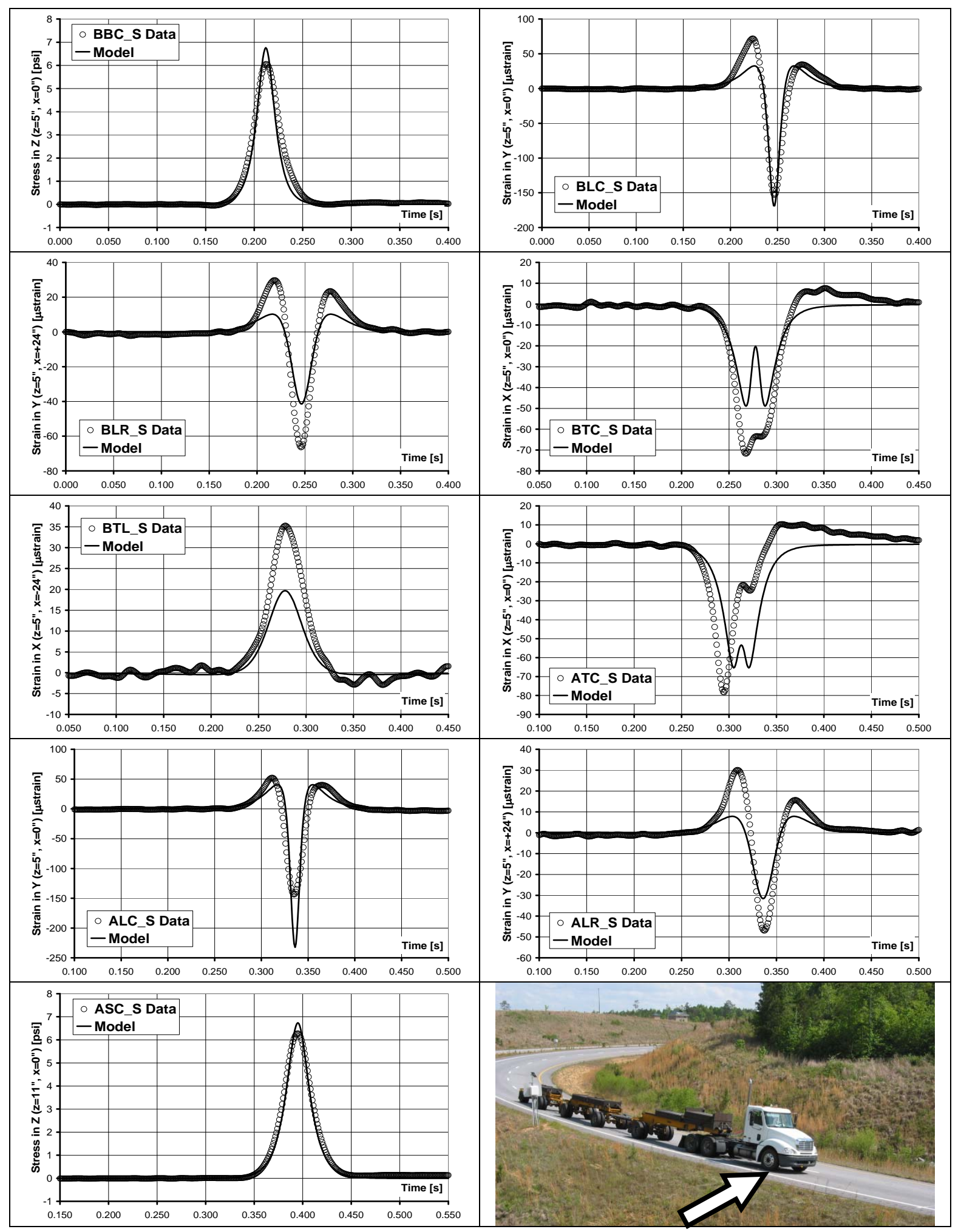

Figure 4.3.6: Calculated and measured N1 responses - right side of steering axle (1S). 


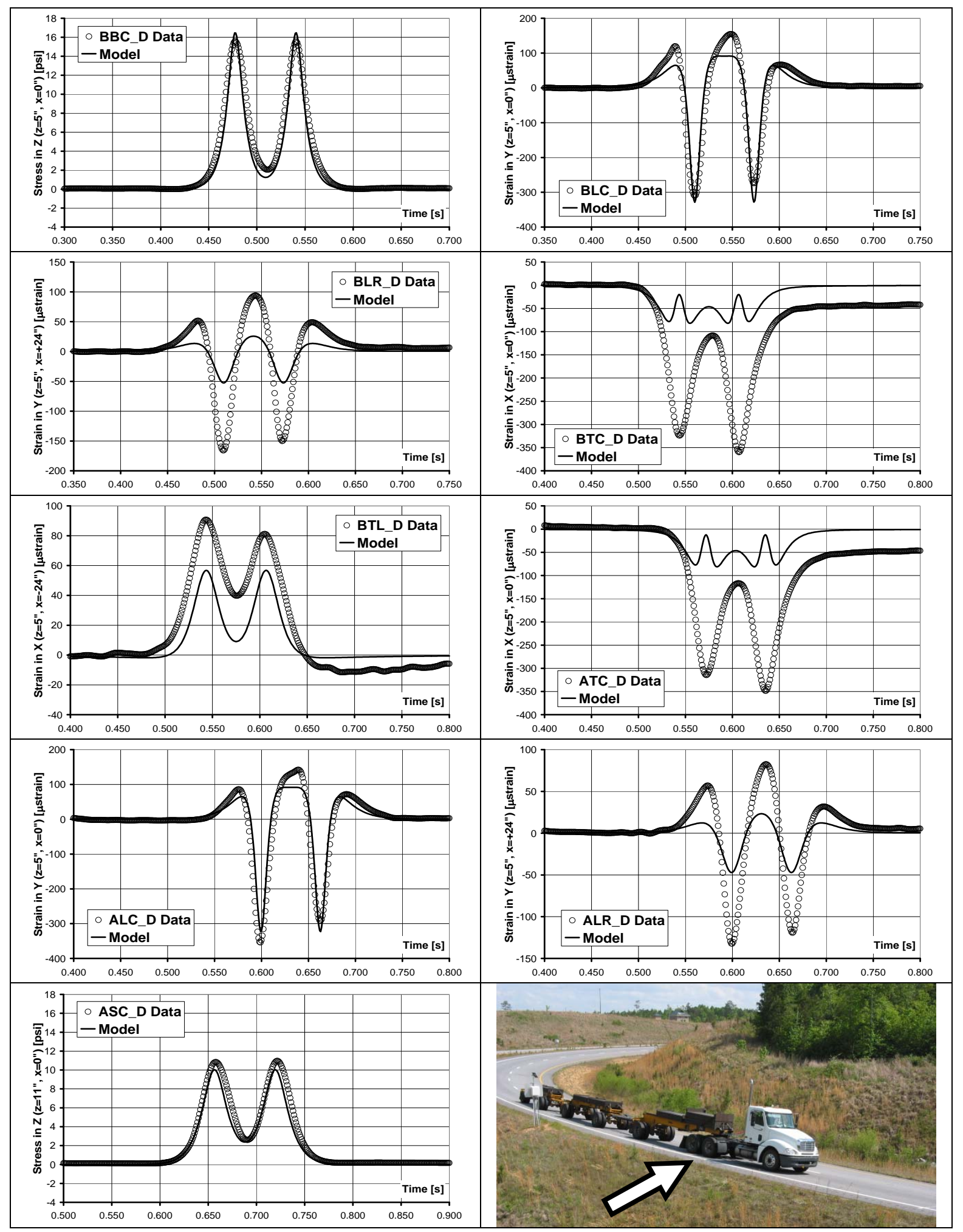

Figure 4.3.7: Calculated and measured N1 responses - right side of drive axle (1D and 2D). 


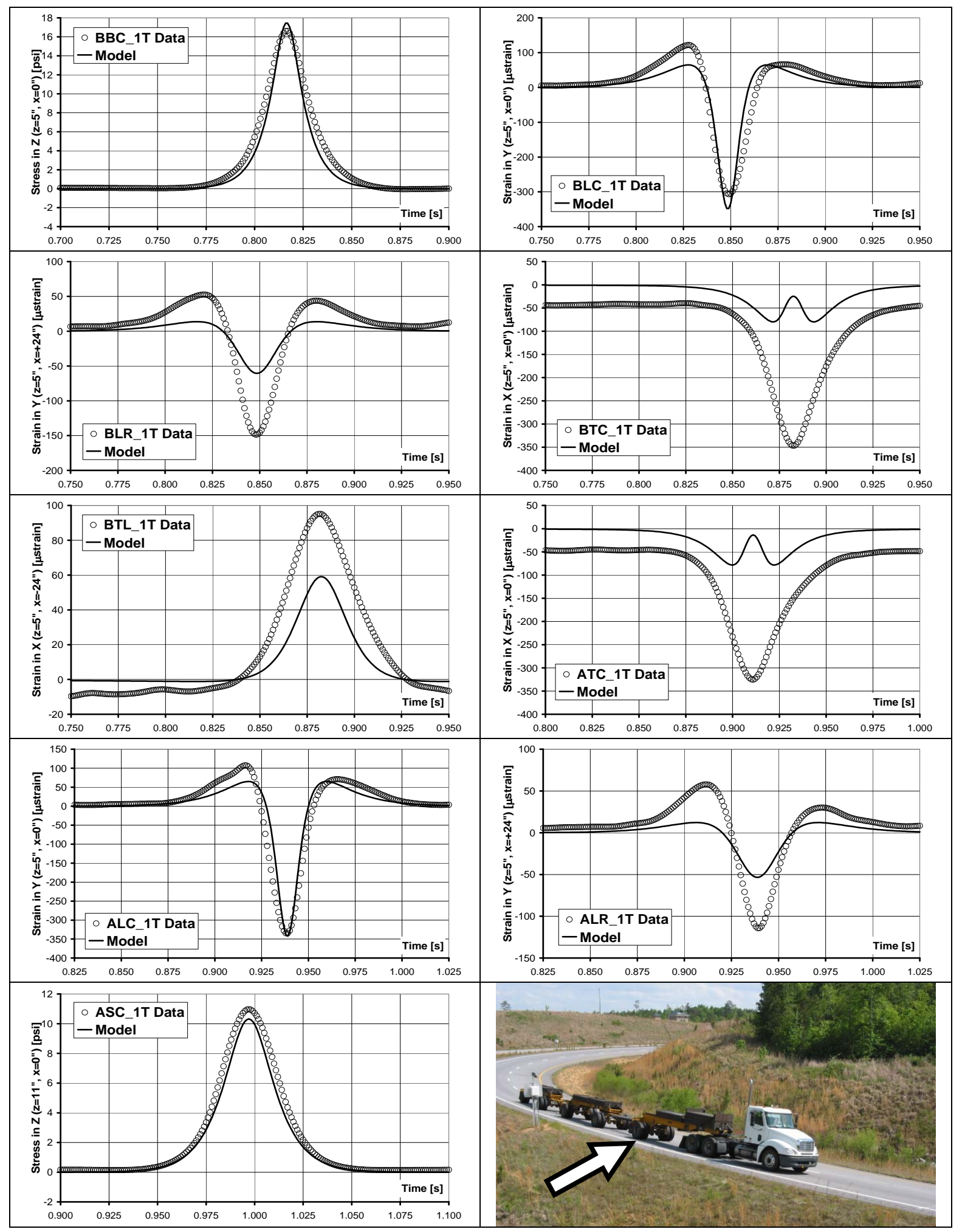

Figure 4.3.8: Calculated and measured N1 responses - right side of first trailer axle (1T). 


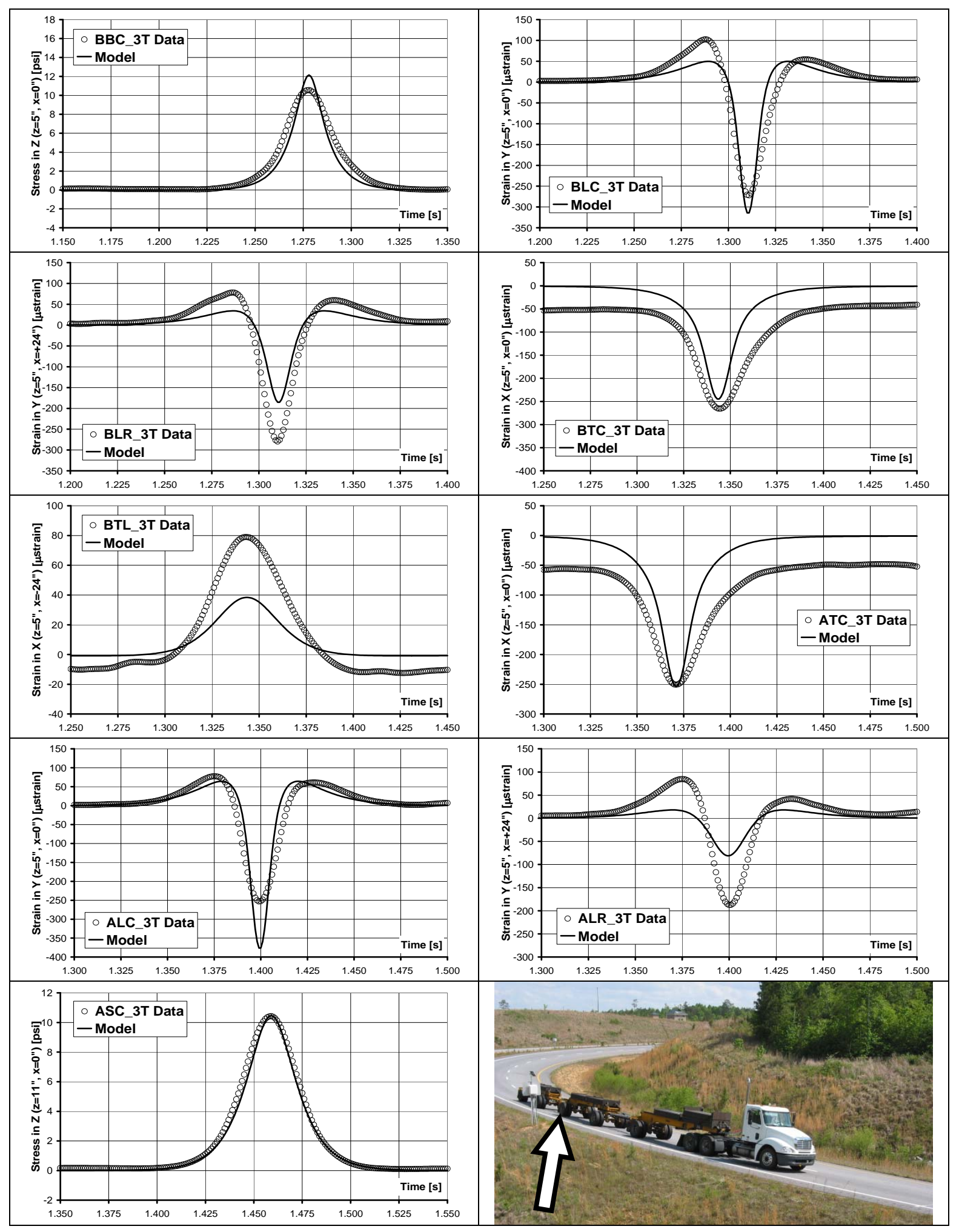

Figure 4.3.9: Calculated and measured N1 responses - right side of third trailer axle (3T). 


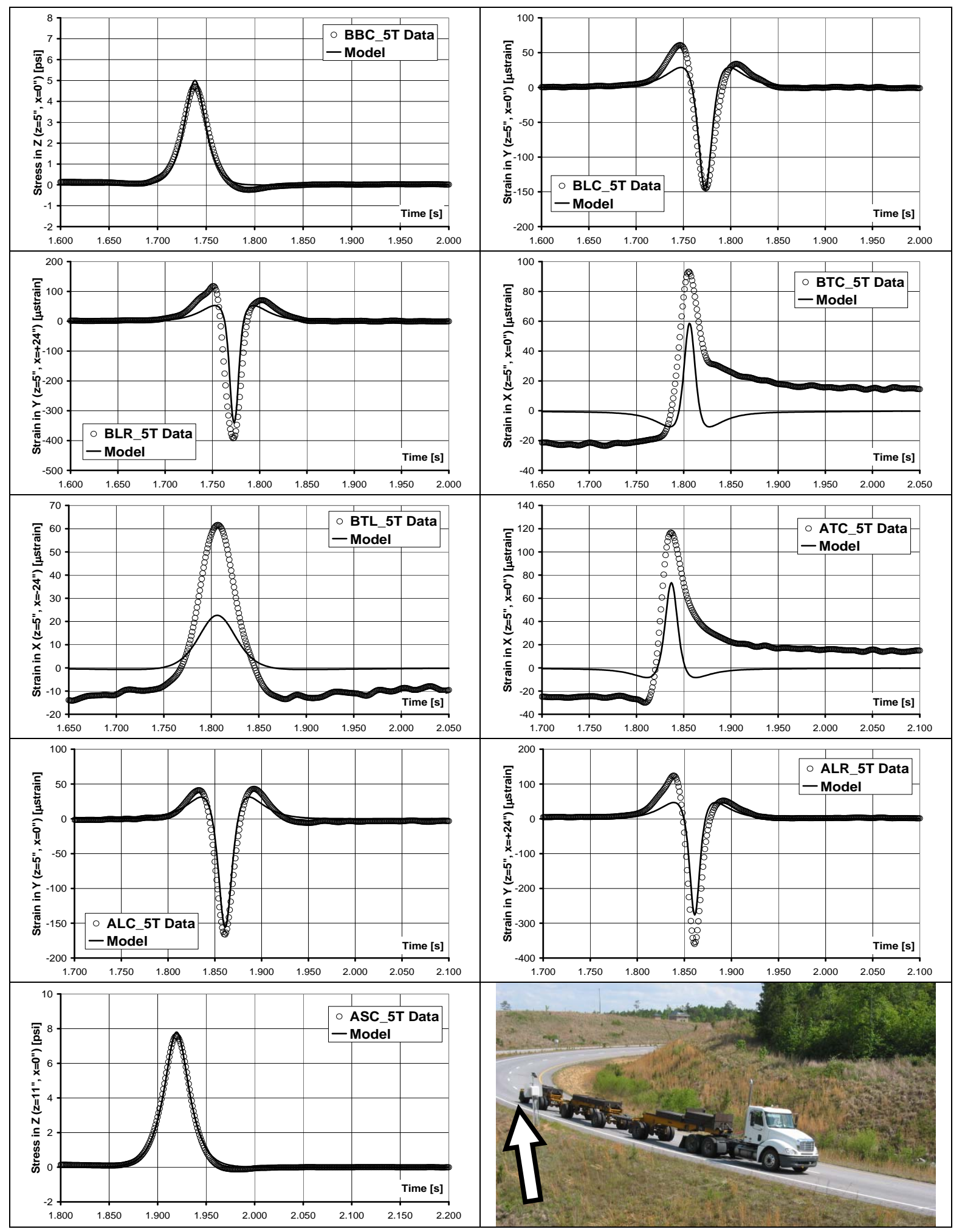

Figure 4.3.10: Calculated and measured N1 responses - right side of last trailer axle (5T). 


\subsection{APPRAISAL OF BASIC ANALYSIS}

This chapter tackled the primary study objective, dealing with: (i) development of a basic mechanistic pavement model based on isotropic LET; (ii) calibration of the layered model using APT data through a process of inverse analysis; (iii) extension of the model capabilities to apply to other loading configurations, other loading speeds, and different environmental conditions; (iv) application of the extended model to simulate the loading and environment at NCAT; and (v) assessment of forecastability.

Analysis of the APT structure was focused on the pavement in the initial phase of the experiment, after 5,000 load applications, and also after 80,000 load applications. The pavement was modeled using isotropic LET with material properties derived through a process of backcalculation. The analysis was performed twice, separately for each experimental stage, using the time history of the all gauge readings collected during one pass of the APT carriage. Overall, the calibrated model captured relatively well the trends in the test data (see Subsection 4.2.3).

Contrasting the backcalculated moduli in the two test stages revealed that the pavement components increased in stiffness during the experiment. Most significant was the increase in base stiffness, which was about 50\% stiffer after 80,000 load applications compared to the initial conditions, followed by about $16 \%$ increase in subgrade modulus. Further densification under load is believed to be the main cause for these changes.

The calibrated APT model was next extended to apply to other experimental conditions by adjusting the stiffness of the HMA to reflect changes in loading speed and temperature compared to the APT experiment. For this purpose the complex modulus data (see Subsection 3.3.2) were further analyzed; resulting in a new set of master curves and time-temperature shifting which were based on the combined properties of the different HMA lifts. Subsequently, changes in HMA temperature were related to modulus adjustments using time-temperature shifting; changes in loading speed were also related to modulus adjustments by manipulating the effective frequency level.

Focusing on the initial phase of the experiment, the extended APT model was then used to forecast load generated responses at NCAT, consisting of: peak FWD 
deflections and stresses and strains induced by a moving truck. Considering all simplifying assumptions made in the aforementioned scheme and the relatively few free parameters used to represent the pavement system, the extended model performed relatively well in projecting resilient responses at NCAT. 


\section{CHAPTER 5 - ADVANCED MECHANISTIC METHODS}

In this chapter two more advanced models compared with the preceding chapter are developed with the aim of establishing a superior link between the APT and NCAT experiments. The first involves LET with anisotropic material properties and the second involves layered viscoelastic theory (LVT) with isotropic material properties. The underlying theories and computational implementations are discussed in subsections 5.1.1 and 5.2.1. In subsections 5.1.2 and 5.2.2 the models are calibrated using the APT experiment; in subsections 5.1.3 and 5.2.3 they are further extended using laboratory data and then applied to forecast selected NCAT responses. A short summary and discussion of findings is provided in Section 5.3.

\subsection{LAYERED ELASTIC ANISOTROPIC MODEL}

\subsubsection{Theory and Computational Implementation}

This subsection contains the derivation of stresses and displacements for the case of transversely isotropic multilayered elastic half-space with vertical loads applied at the surface spread evenly over a circle. In terms of a Cartesian coordinate system $(x, y, z)$, with $x-y$ as the plane of material isotropy, the constitutive law is:

$$
\begin{aligned}
& \varepsilon_{x}=a_{11} \cdot \sigma_{x}+a_{12} \cdot \sigma_{y}+a_{13} \cdot \sigma_{z} \\
& \varepsilon_{y}=a_{12} \cdot \sigma_{x}+a_{11} \cdot \sigma_{y}+a_{13} \cdot \sigma_{z} \\
& \varepsilon_{z}=a_{13} \cdot \sigma_{x}+a_{13} \cdot \sigma_{y}+a_{33} \cdot \sigma_{z} \\
& \varepsilon_{y z}=\left(a_{44} / 2\right) \cdot \tau_{y z} \\
& \varepsilon_{x z}=\left(a_{44} / 2\right) \cdot \tau_{x z} \\
& \varepsilon_{x y}=\left(a_{11}-a_{12}\right) \cdot \tau_{x y} \ldots \ldots \ldots \ldots \ldots \ldots \ldots \ldots \ldots \ldots \ldots \ldots \ldots \ldots \ldots \ldots \ldots
\end{aligned}
$$

in which the six $\varepsilon$ 's are components of the strain tensor, the $\sigma$ 's and the $\tau$ 's are components of the stress tensor, and the $a$ 's are the material properties (i.e., elastic constants). 
In terms of a cylindrical coordinate system $(r, \theta, z)$, with $\mathrm{z}$ as the axis of material symmetry, and assuming an axially symmetric deformation field (i.e., $\left.\varepsilon_{z \theta}=\varepsilon_{r \theta}=0\right)$, the constitutive law becomes:

$$
\begin{aligned}
& \varepsilon_{r}=a_{11} \cdot \sigma_{r}+a_{12} \cdot \sigma_{\theta}+a_{13} \cdot \sigma_{z} \\
& \varepsilon_{\theta}=a_{12} \cdot \sigma_{r}+a_{11} \cdot \sigma_{\theta}+a_{13} \cdot \sigma_{z} \\
& \varepsilon_{z}=a_{13} \cdot \sigma_{r}+a_{13} \cdot \sigma_{\theta}+a_{33} \cdot \sigma_{z} \\
& \varepsilon_{r z}=\left(a_{44} / 2\right) \cdot \tau_{r z}
\end{aligned}
$$

in which $a_{11}=1 / E_{x}, \quad a_{33}=1 / E_{z}, \quad a_{12}=-v_{x y} / E_{x}, \quad a_{13}=-v_{z x} / E_{z}$ and $a_{44}=1 / G_{x z}$. Hence, five elastic constants are included, namely: two Young's moduli $E_{x}\left(=E_{y}\right)$ and $E_{z}$; two Poisson's ratios $v_{x y}\left(=v_{y x}\right)$ and $v_{z x}\left(=v_{x z} \cdot E_{z} / E_{x}\right)$; and one shear modulus $G_{x z}\left(=G_{y z}\right)$. The condition that the strain energy must be positive imposes the following property restrictions (PRs) on the values of the elastic constants (e.g., Poulus and Davis, 1974): (PR1) $E_{x}, E_{z}, G_{x z}>0$; (PR2) $1-v_{x y}-2 \cdot v_{x z} \cdot v_{z x}>0$; and (PR3) $1-\left|v_{x y}\right|>0$.

Following Lekhnitskii (1963) and Singh (1986), the stresses $\left(\sigma_{r}, \sigma_{\theta}, \sigma_{z}, \tau_{r z}\right)$ and displacements ( $u, w$ in the $r, z$ directions respectively) can be derived from a stress function $\phi(r, z)$ as follows:

$$
\begin{aligned}
& \sigma_{r}=-\frac{\partial}{\partial z}\left(\frac{\partial \phi^{2}}{\partial r^{2}}+\frac{b}{r} \cdot \frac{\partial \phi}{\partial r}+a \cdot \frac{\partial \phi^{2}}{\partial z^{2}}\right) \\
& \sigma_{\theta}=-\frac{\partial}{\partial z}\left(b \cdot \frac{\partial \phi^{2}}{\partial r^{2}}+\frac{1}{r} \cdot \frac{\partial \phi}{\partial r}+a \cdot \frac{\partial \phi^{2}}{\partial z^{2}}\right) \\
& \sigma_{z}=\frac{\partial}{\partial z}\left(c \cdot \frac{\partial \phi^{2}}{\partial r^{2}}+\frac{c}{r} \cdot \frac{\partial \phi}{\partial r}+d \cdot \frac{\partial \phi^{2}}{\partial z^{2}}\right) \\
& \tau_{r z}=\frac{\partial}{\partial r}\left(\frac{\partial \phi^{2}}{\partial r^{2}}+\frac{1}{r} \cdot \frac{\partial \phi}{\partial r}+a \cdot \frac{\partial \phi^{2}}{\partial z^{2}}\right)
\end{aligned}
$$




$$
\begin{aligned}
& u=\left(a_{12}-a_{11}\right) \cdot(1-b) \cdot \frac{\partial^{2} \phi}{\partial r \partial z} \\
& w=\left(2 \cdot a_{13} \cdot a-a_{33} \cdot d\right) \cdot \frac{\partial^{2} \phi}{\partial z^{2}}-a_{44}\left(\frac{\partial \phi^{2}}{\partial r^{2}}+\frac{1}{r} \cdot \frac{\partial \phi}{\partial r}\right)
\end{aligned}
$$

in which the parameters $a, b, c$ and $d$ are functions of the elastic constants:

$$
\begin{aligned}
& a=\frac{a_{13} \cdot\left(a_{11}-a_{12}\right)}{a_{11} \cdot a_{33}-a_{13}^{2}} \\
& b=\frac{a_{13} \cdot\left(a_{13}+a_{44}\right)-a_{12} \cdot a_{33}}{a_{11} \cdot a_{33}-a_{13}^{2}} \\
& c=\frac{a_{13} \cdot\left(a_{11}-a_{12}\right)+a_{11} \cdot a_{44}}{a_{11} \cdot a_{33}-a_{13}^{2}} \\
& d=\frac{a_{11}^{2}-a_{12}^{2}}{a_{11} \cdot a_{33}-a_{13}^{2}}
\end{aligned}
$$

and the stress function $\phi(r, z)$ satisfies the 'compatibility' equation $\nabla_{\alpha}^{2} \nabla_{\beta}^{2} \phi=0$ in which $\nabla_{\alpha}^{2}=\partial^{2} / \partial r^{2}+r^{-1} \cdot \partial / \partial r+\alpha^{-2} \cdot \partial^{2} / \partial z^{2}$ and $\nabla_{\beta}^{2}$ is identical to $\nabla_{\alpha}^{2}$ except for $\beta$ in place of $\alpha$. The terms $\alpha$ and $\beta$ are also derived from the material properties, as follows:

$$
\left(\begin{array}{l}
\alpha^{2} \\
\beta^{2}
\end{array}\right)=\frac{a+c \pm \sqrt{(a+c)^{2}-4 \cdot d}}{2 \cdot d}
$$

The above algorithm imposes additional restrictions (named algorithm restrictions or ARs) on the material properties: (AR1) $a_{11} \cdot a_{33}-a_{13}^{2} \neq 0$; (AR2) $(a+c)^{2}-4 \cdot d>0$; (AR3) $\alpha^{2}>0$; and (AR4) $\beta^{2}>0$. In addition, $\alpha$ and $\beta$ must be distinct or the following derivation becomes singular; for this reason the isotropic case, in which $\alpha=\beta=1$, can only be approached but not directly computed.

Consider a semi-infinite medium made of $n-1$ parallel layers lying over a halfspace. Each layer is identified by a subscript $i$ with material properties $\left(E_{z}\right)_{i},\left(E_{x}\right)_{i}$, 
$\left(v_{z x}\right)_{i},\left(v_{x y}\right)_{i}$ and $\left(G_{x z}\right)_{i}$. Layers are numbered serially, the layer at the top being layer 1 and the half-space, layer $n$. Similar to the isotropic case (see Subsection 4.2.1), the origin of the cylindrical coordinate system is placed at the surface of the first layer with the $z$-axis pointing into the medium and the $r$-axis parallel to the layers. As before, the depth to the individual interfaces (measured from the surface) is denoted by $Z_{i}$ $(i=1,2, . . n-1)$. Hence, $z_{1}$ is the thickness of layer $1, z_{2}$ is the combined thickness of layers 1 and 2, and so on. The combined thickness of the $n-1$ layers is denoted by $H$ (i.e., $\left.H=z_{n-1}\right)$.

Inspired by Huang (2004) and Lekhnitskii (1963), a stress function that complies with all of the above requirements is offered:

$$
\phi_{i}(\rho, \lambda)=\left(\frac{H^{3} \cdot J_{0}(m \cdot \rho)}{m^{2}}\right) \cdot\left(\begin{array}{l}
A_{i} \cdot e^{-m \cdot \alpha_{i} \cdot\left(\lambda_{i}-\lambda\right)}+B_{i} \cdot e^{-m \cdot \alpha_{i} \cdot\left(\lambda-\lambda_{i-1}\right)} \\
+C_{i} \cdot e^{-m \cdot \beta_{i} \cdot\left(\lambda_{i}-\lambda\right)}+D_{i} \cdot e^{-m \cdot \beta_{i} \cdot\left(\lambda-\lambda_{i-1}\right)}
\end{array}\right)
$$

in which $\rho=r / H, \lambda=z / H, \lambda_{i}=z_{i} / H$ and $m$ is a unitless parameter; $A_{i}, B_{i}, C_{i}$ and $D_{i}$ are all unitless functions of $m ; J_{k}$ denotes a Bessel function of the first kind of order $k$; and the subscript $i$ refers to the layer number. Substitution of this equation into equations 5.1.3a-f yields the responses of interest in a given layer $i$ due to a vertical non-dimensional surface load of the form $m \cdot J_{0}(m \cdot \rho)$ and not due to a uniform load distributed over a circular area (this fact is indicated by an asterisk). The resulting expressions are presented in what follows for completeness of the derivation.

$$
\begin{aligned}
& \left(\sigma_{r}^{*}\right)_{i}=\left(\begin{array}{l}
\alpha_{i} \cdot L_{i}^{\alpha} \cdot\left(A_{i} \cdot e^{-m \cdot \alpha_{i} \cdot\left(\lambda_{i}-\lambda\right)}-B_{i} \cdot e^{-m \cdot \alpha_{i} \cdot\left(\lambda-\lambda_{i-1}\right)}\right) \\
+\beta_{i} \cdot L_{i}^{\beta} \cdot\left(C_{i} \cdot e^{-m \cdot \beta_{i} \cdot\left(\lambda_{i}-\lambda\right)}-D_{i} \cdot e^{-m \cdot \beta_{i} \cdot\left(\lambda-\lambda_{i-1}\right)}\right)
\end{array}\right) \cdot m \cdot J_{0}(m \cdot \rho) \\
& +\left(\begin{array}{l}
\alpha_{i} \cdot\left(A_{i} \cdot e^{-m \cdot \alpha_{i} \cdot\left(\lambda_{i}-\lambda\right)}-B_{i} \cdot e^{-m \cdot \alpha_{i} \cdot\left(\lambda-\lambda_{i-1}\right)}\right) \\
+\beta_{i} \cdot\left(C_{i} \cdot e^{-m \cdot \beta_{i} \cdot\left(\lambda_{i}-\lambda\right)}-D_{i} \cdot e^{-m \cdot \beta_{i} \cdot\left(\lambda-\lambda_{i-1}\right)}\right)
\end{array}\right) \cdot \frac{\left(b_{i}-1\right) \cdot J_{1}(m \cdot \rho)}{\rho} \\
& \left(\sigma_{\theta}^{*}\right)_{i}=\left(\begin{array}{l}
Q_{i}^{\alpha} \cdot\left(A_{i} \cdot e^{-m \cdot \alpha_{i} \cdot\left(\lambda_{i}-\lambda\right)}-B_{i} \cdot e^{-m \cdot \alpha_{i} \cdot\left(\lambda-\lambda_{i-1}\right)}\right) \\
+Q_{i}^{\beta} \cdot\left(C_{i} \cdot e^{-m \cdot \beta_{i} \cdot\left(\lambda_{i}-\lambda\right)}-D_{i} \cdot e^{-m \cdot \beta_{i} \cdot\left(\lambda-\lambda_{i-1}\right)}\right)
\end{array}\right) \cdot m \cdot J_{0}(m \cdot \rho) \\
& +\left(\begin{array}{l}
S_{i}^{\alpha} \cdot\left(A_{i} \cdot e^{-m \cdot \alpha_{i} \cdot\left(\lambda_{i}-\lambda\right)}-B_{i} \cdot e^{-m \cdot \alpha_{i} \cdot\left(\lambda-\lambda_{i-1}\right)}\right) \\
+S_{i}^{\alpha} \cdot\left(C_{i} \cdot e^{-m \cdot \beta_{i} \cdot\left(\lambda_{i}-\lambda\right)}-D_{i} \cdot e^{-m \cdot \beta_{i} \cdot\left(\lambda-\lambda_{i-1}\right)}\right)
\end{array}\right) \cdot \frac{J_{1}(m \cdot \rho)}{\rho}
\end{aligned}
$$




$$
\begin{aligned}
& \left(\sigma_{z}^{*}\right)_{i}=m \cdot J_{0}(m \cdot \rho) \cdot\left(\begin{array}{l}
K_{i}^{\alpha} \cdot\left(A_{i} \cdot e^{-m \cdot \alpha_{i} \cdot\left(\lambda_{i}-\lambda\right)}-B_{i} \cdot e^{-m \cdot \alpha_{i} \cdot\left(\lambda-\lambda_{i-1}\right)}\right) \\
+K_{i}^{\beta} \cdot\left(C_{i} \cdot e^{-m \cdot \beta_{i} \cdot\left(\lambda_{i}-\lambda\right)}-D_{i} \cdot e^{-m \cdot \beta_{i} \cdot\left(\lambda-\lambda_{i-1}\right)}\right)
\end{array}\right) \cdots \\
& \left(\begin{array}{l}
\left.\tau_{r z}^{*}\right)_{i}=m \cdot J_{1}(m \cdot \rho) \cdot\left(\begin{array}{l}
L_{i}^{\alpha} \cdot\left(A_{i} \cdot e^{-m \cdot \alpha_{i} \cdot\left(\lambda_{i}-\lambda\right)}+B_{i} \cdot e^{-m \cdot \alpha_{i} \cdot\left(\lambda-\lambda_{i-1}\right)}\right) \\
+L_{i}^{\beta} \cdot\left(C_{i} \cdot e^{-m \cdot \beta_{i} \cdot\left(\lambda_{i}-\lambda\right)}+D_{i} \cdot e^{-m \cdot \beta_{i} \cdot\left(\lambda-\lambda_{i-1}\right)}\right)
\end{array}\right) \cdots \cdots \\
\left(u^{*}\right)_{i}=H \cdot F_{i} \cdot J_{1}(m \cdot \rho) \cdot\left(\begin{array}{l}
\alpha_{i} \cdot\left(A_{i} \cdot e^{-m \cdot \alpha_{i} \cdot\left(\lambda_{i}-\lambda\right)}-B_{i} \cdot e^{-m \cdot \alpha_{i} \cdot\left(\lambda-\lambda_{i-1}\right)}\right) \\
+\beta_{i} \cdot\left(C_{i} \cdot e^{-m \cdot \beta_{i} \cdot\left(\lambda_{i}-\lambda\right)}-D_{i} \cdot e^{-m \cdot \beta_{i} \cdot\left(\lambda-\lambda_{i-1}\right)}\right)
\end{array}\right)
\end{array}\right) \\
& \left(w^{*}\right)_{i}=H \cdot J_{0}(m \cdot \rho) \cdot\left(\begin{array}{l}
G_{i}^{\alpha} \cdot\left(A_{i} \cdot e^{-m \cdot \alpha_{i} \cdot\left(\lambda_{i}-\lambda\right)}+B_{i} \cdot e^{-m \cdot \alpha_{i} \cdot\left(\lambda-\lambda_{i-1}\right)}\right) \\
+G_{i}^{\beta} \cdot\left(C_{i} \cdot e^{-m \cdot \beta_{i} \cdot\left(\lambda_{i}-\lambda\right)}+D_{i} \cdot e^{-m \cdot \beta_{i} \cdot\left(\lambda-\lambda_{i-1}\right)}\right)
\end{array}\right) \cdots
\end{aligned}
$$

in which the following settings were used in order to save space:

$$
\begin{aligned}
& L_{i}^{\alpha}=1-a_{i} \cdot \alpha_{i}^{2} \\
& L_{i}^{\beta}=1-a_{i} \cdot \beta_{i}^{2} \\
& Q_{i}^{\alpha}=\alpha_{i} \cdot\left(b_{i}-a_{i} \cdot \alpha_{i}^{2}\right) \\
& Q_{i}^{\beta}=\beta_{i} \cdot\left(b_{i}-a_{i} \cdot \beta_{i}^{2}\right) \\
& S_{i}^{\alpha}=\alpha_{i} \cdot\left(1-b_{i}\right) \\
& S_{i}^{\beta}=\beta_{i} \cdot\left(1-b_{i}\right)
\end{aligned}
$$$$
K_{i}^{\alpha}=d_{i} \cdot \alpha_{i}^{3}-c_{i} \cdot \alpha_{i}
$$$$
K_{i}^{\beta}=d_{i} \cdot \beta_{i}^{3}-c_{i} \cdot \beta_{i}
$$

$$
G_{i}^{\beta}=\left(a_{44}\right)_{i}-\beta_{i}^{2} \cdot\left(\left(a_{33}\right)_{i} \cdot d_{i}-2 \cdot\left(a_{13}\right)_{i} \cdot a_{i}\right)
$$

The value of each of the functions $A_{i}(m), B_{i}(m), C_{i}(m)$ and $D_{i}(m)$ in equations 5.1.7a-f is determined, for any given $m$, by solving a set of linear equations that represent the boundary conditions. For the lowest layer, where $i=n$ and $\lambda \rightarrow \infty$, 
the stresses and displacements must vanish; this leads to $A_{n}=C_{n}=0$. For a vertical load of the form $m \cdot J_{0}(m \cdot \rho)$ applied to the surface of layer 1 (i.e., $i=1, \lambda=0$ ), and in the absence of shearing forces, we obtain the two conditions: $\left(\sigma_{z}^{*}\right)_{1}=m \cdot J_{0}(m \cdot \rho)$ and $\left(\tau_{r z}^{*}\right)_{1}=0$. Using equations $5.1 .7 \mathrm{c}$ and $5.1 .7 \mathrm{~d}$ these conditions can be written explicitly as follows:

$$
\begin{aligned}
& K_{1}^{\alpha} \cdot\left(A_{1} \cdot e^{-m \cdot \alpha_{1} \cdot \lambda_{1}}-B_{1}\right)+K_{1}^{\beta} \cdot\left(C_{1} \cdot e^{-m \cdot \beta_{1} \cdot \lambda_{1}}-D_{1}\right)=1 \\
& L_{1}^{\alpha} \cdot\left(A_{1} \cdot e^{-m \cdot \alpha_{1} \cdot \lambda_{1}}+B_{1}\right)+L_{1}^{\beta} \cdot\left(C_{1} \cdot e^{-m \cdot \beta_{1} \cdot \lambda_{1}}+D_{1}\right)=0
\end{aligned}
$$

Continuity of vertical and shear stresses at the interface between layers $i$ and $i+1$ when $\lambda=\lambda_{i}$, is represented by the equations $\left(\sigma_{z}^{*}\right)_{i}=\left(\sigma_{z}^{*}\right)_{i+1}$ and $\left(\tau_{r z}^{*}\right)_{i}=\left(\tau_{r z}^{*}\right)_{i+1}$ or more explicitly:

$$
\begin{aligned}
& \left(\begin{array}{l}
K_{i}^{\alpha} \cdot\left(A_{i}-B_{i} \cdot e^{-m \cdot \alpha_{i} \cdot\left(\lambda_{i}-\lambda_{i-1}\right)}\right) \\
+K_{i}^{\beta} \cdot\left(C_{i}-D_{i} \cdot e^{-m \cdot \beta_{i} \cdot\left(\lambda_{i}-\lambda_{i-1}\right)}\right)
\end{array}\right)=\left(\begin{array}{l}
K_{i+1}^{\alpha} \cdot\left(A_{i+1} \cdot e^{-m \cdot \alpha_{i+1} \cdot\left(\lambda_{i+1}-\lambda_{i}\right)}-B_{i+1}\right) \\
+K_{i+1}^{\beta} \cdot\left(C_{i+1} \cdot e^{-m \cdot \beta_{i+1} \cdot\left(\lambda_{i+1}-\lambda_{i}\right)}-D_{i+1}\right)
\end{array}\right) \\
& \left(\begin{array}{l}
L_{i}^{\alpha} \cdot\left(A_{i}+B_{i} \cdot e^{-m \cdot \alpha_{i} \cdot\left(\lambda_{i}-\lambda_{i-1}\right)}\right) \\
+L_{i}^{\beta} \cdot\left(C_{i}+D_{i} \cdot e^{-m \cdot \beta_{i} \cdot\left(\lambda_{i}-\lambda_{i-1}\right)}\right)
\end{array}\right)=\left(\begin{array}{l}
L_{i+1}^{\alpha} \cdot\left(A_{i+1} \cdot e^{-m \cdot \alpha_{i+1} \cdot\left(\lambda_{i+1}-\lambda_{i}\right)}+B_{i+1}\right) \\
+L_{i+1}^{\beta} \cdot\left(C_{i+1} \cdot e^{-m \cdot \beta_{i+1} \cdot\left(\lambda_{i+1}-\lambda_{i}\right)}+D_{i+1}\right)
\end{array}\right)
\end{aligned}
$$

Continuity of vertical and radial displacements at the interface between layers $i$ and $i+1$ when $\lambda=\lambda_{i}$, is represented by the equations $\left(w^{*}\right)_{i}=\left(w^{*}\right)_{i+1}$ and $\left(u^{*}\right)_{i}=\left(u^{*}\right)_{i+1}$ or explicitly:

$$
\begin{aligned}
& \left\{\begin{array}{l}
G_{i}^{\alpha} \cdot\left(A_{i}+B_{i} \cdot e^{-m \cdot \alpha_{i} \cdot\left(\lambda_{i}-\lambda_{i-1}\right)}\right) \\
+G_{i}^{\beta} \cdot\left(C_{i}+D_{i} \cdot e^{-m \cdot \beta_{i} \cdot\left(\lambda_{i}-\lambda_{i-1}\right)}\right)
\end{array}\right\}=\left\{\begin{array}{l}
G_{i+1}^{\alpha} \cdot\left(A_{i+1} \cdot e^{-m \cdot x_{i+1} \cdot\left(\lambda_{i+1}-\lambda_{i}\right)}+B_{i+1}\right) \\
+G_{i+1}^{\beta} \cdot\left(C_{i+1} \cdot e^{-m \cdot \beta_{i+1} \cdot\left(\lambda_{i+1}-\lambda_{i}\right)}+D_{i+1}\right)
\end{array}\right\} \cdots \ldots . . \\
& \frac{F_{i}}{F_{i+1}} \cdot\left\{\begin{array}{l}
\alpha_{i} \cdot\left(A_{i}-B_{i} \cdot e^{-m \cdot \alpha_{i} \cdot\left(\lambda_{i}-\lambda_{i-1}\right)}\right) \\
+\beta_{i} \cdot\left(C_{i}-D_{i} \cdot e^{-m \cdot \beta_{i} \cdot\left(\lambda_{i}-\lambda_{i-1}\right)}\right)
\end{array}\right\}=\left\{\begin{array}{l}
\alpha_{i+1} \cdot\left(A_{i+1} \cdot e^{-m \cdot \alpha_{i+1} \cdot\left(\lambda_{i+1}-\lambda_{i}\right)}-B_{i+1}\right) \\
+\beta_{i+1} \cdot\left(C_{i+1} \cdot e^{-m \cdot \beta_{i+1} \cdot\left(\lambda_{i+1}-\lambda_{i}\right)}-D_{i+1}\right)
\end{array}\right\} .
\end{aligned}
$$

Note that equation 5.1.9f represents full bonding at the interface between layers $i$ and $i+1$. Finally, the response of interest $R$ generated by a uniform load $q$ distributed over a circular area of radius $\bar{a}$ is obtained by performing the integration: 


$$
R=(q \cdot \alpha) \cdot \int_{m=0}^{\infty} \frac{R^{*}}{m} \cdot J_{1}(m \cdot \alpha) \cdot d m
$$

in which $\alpha=\bar{a} / H$ and $R^{*}$ is any stress or displacement of interest from equations 5.1.7a-f. Thereafter, the strains are calculated from equations 5.1.2a-d.

The aforementioned derivation was programmed into an Excel worksheet (see program ELLEA2 in Appendix B). Similar to the isotropic program ELLEA1 (see Chapter 4 and also Appendix B), the case of fully bonded five layers and two independent loaded areas was considered. The integration method for equation 5.1.10 was also similar to that used for equation 4.2.5. The program was verified against three cases: (i) closed form solution for a transversely-isotropic half-space acted upon by a concentrated force applied at the surface (Lekhnitskii, 1963); (ii) tabulated solution for a transversely-isotropic half-space due to a distributed load over a circular area (Poulos and Davis, 1974); and (iii) numerical solution for isotropic LET (De Jong et al., 1973) when the transversely-isotropic material properties approach the isotropic case.

The program's user interface is shown in Figure 5.1.1. As can be seen, the structural information is located in the topmost table. For each layer six attributes are needed, namely the thickness and the five elastic constants. The loading information is located in the following table; two loads are considered, each requiring four input values: the stress intensity $q$, the radius $\bar{a}$, and two relative coordinates in $\mathrm{X}$ and in $\mathrm{Y}$ identifying the location of application. The computational results are shown in the bottom table. Negative values for stresses or strains (or both) in the $\mathrm{X}, \mathrm{Y}$ and $\mathrm{Z}$ directions indicate tension while positive values indicate compression; positive displacement refers to a downward deflection while a negative displacement refers to heaving. Similar to ELLEA1, the calculations are done in real-time which means that any change in the input parameters is automatically reflected in the results. It should be noted that the program also calculates and displays (separately for each layer), property restrictions 2 and 3 (i.e., PR2 and PR3) and algorithm restrictions 2, 3 and 4 (i.e., AR2, AR3 and AR4). For the example in Figure 5.1.1 these are shown separately in Figure 5.1.2. In ELLEA2 these restrictions are displayed to the right hand side of the topmost input table. 


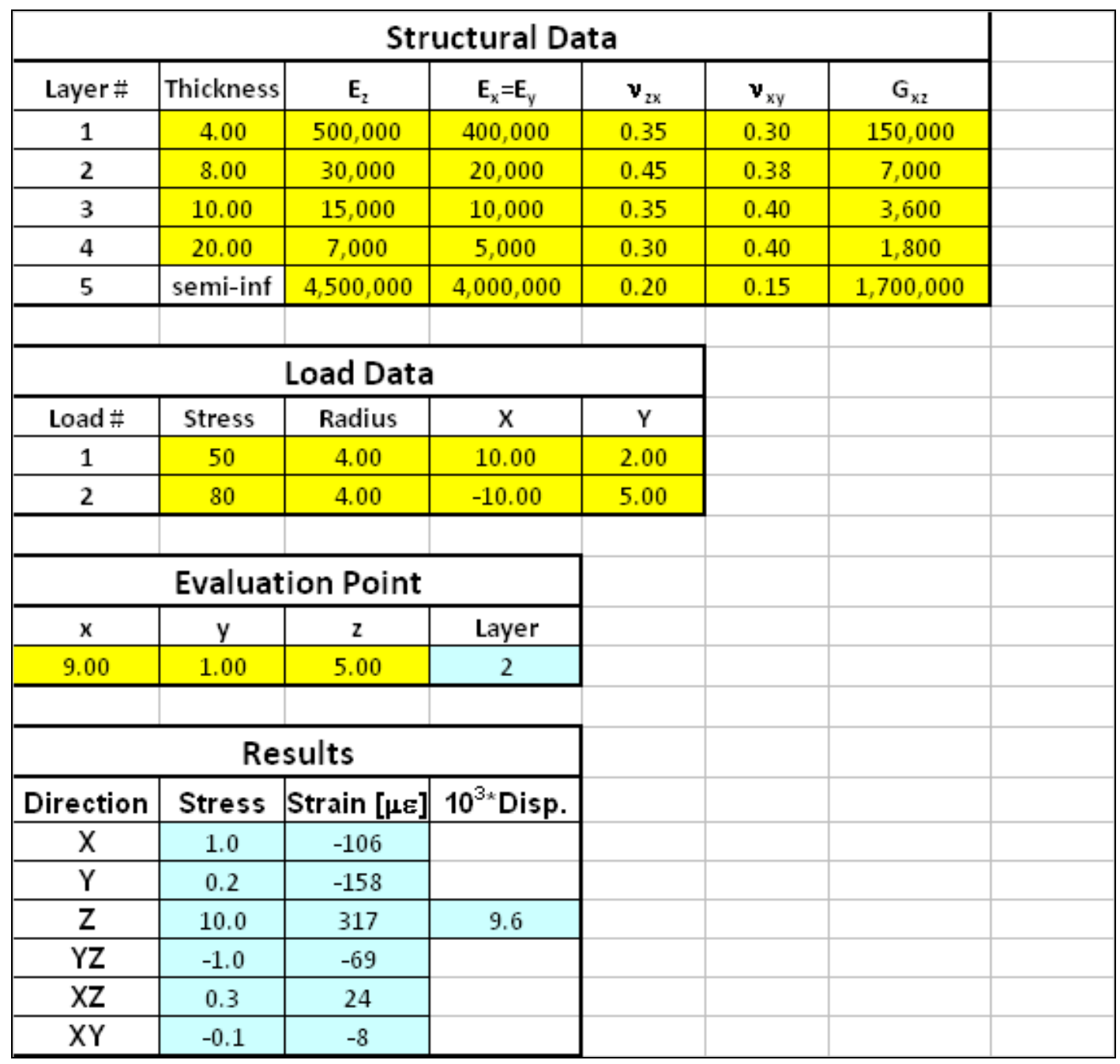

Figure 5.1.1: User interface of the anisotropic LET program ELLEA2.

\begin{tabular}{|c|c|c|c|c|}
\hline \multicolumn{2}{|c|}{ Property Restrictions } & \multicolumn{3}{c|}{ Algorithm Restrictions } \\
\hline PR2: $1-v_{x y}-2^{*} E_{x} / E_{2}^{*}\left(v_{2 x}\right)^{2}>0$ & PR3: $1-a b s\left(v_{x y}\right)>0$ & AR2: $(a+c)^{2}-4 d>0$ & AR3: $\alpha^{2}>0$ & AR4: $\beta^{2}>0$ \\
\hline 0.504 & 0.70 & 2.174 & 1.650 & 0.481 \\
\hline 0.350 & 0.62 & 6.447 & 2.041 & 0.330 \\
\hline 0.437 & 0.60 & 6.553 & 2.197 & 0.332 \\
\hline 0.471 & 0.60 & 5.590 & 2.237 & 0.356 \\
\hline 0.779 & 0.85 & 0.581 & 1.329 & 0.660 \\
\hline
\end{tabular}

Figure 5.1.2: ELLEA2 display of property and algorithm restriction for the example shown in Figure 5.1.1. 


\subsubsection{Calibration to APT Conditions}

The anisotropic LET properties were backcalculated using the APT study. This was performed for the initial stages of the experiment, during pass number 5,000. Similar to the isotropic analysis, a four layered structure was assumed with a semi-infinite (isotropic) concrete bottom. The properties of the HMA $(i=1)$, aggregate base $(i=2)$ and subgrade $(i=3)$ were obtained by matching model generated responses with measured responses. Calculation steps used for this purpose were identical to those outlined in Subsection 4.2.2 except for the number of unknowns. In the most general case, since each anisotropic layer is characterized by five independent elastic properties, there are 15 unknowns to be determined. In order to simplify matters and reduce the number of free parameters to a more realistic level, only the vertical and horizontal moduli were backcalculated for each layer, namely $\left(E_{z}\right)_{i}$ and $\left(E_{x}\right)_{i}$.

The numerical values of the remaining elastic constants were a priori assumed. The shear modulus $G_{x z}$ in each layer was related to the other elastic properties according to the following expression (Wolf, 1935; Barden, 1963; Christian, 1968):

$$
G_{x z}=\frac{E_{x} \cdot E_{z}}{E_{z}+E_{x} \cdot\left(1+2 \cdot v_{z x}\right)}
$$

Also, the two Poisson's ratios were assumed to be identical, i.e., $\left(v_{z x}\right)_{i}=\left(v_{x y}\right)_{i}=v_{i}$ with values predetermined similar to the isotropic case (see Table 4.2.1). This latter assumption is also made in the Australian pavement design guide (Austroads, 2004). Other assumptions are known to exist, most noteworthy of which (although not applied herein) was developed by Graham and Houlsby (1983):

$$
\begin{aligned}
& v_{x y}=v_{z x} \cdot \sqrt{\frac{E_{x}}{E_{z}}} \ldots \\
& G_{x z}=\frac{\sqrt{E_{x} \cdot E_{z}}}{2 \cdot\left(1+v_{x y}\right)}
\end{aligned}
$$


in which it can be seen that the two Poisson's ratios $v_{x y}$ and $v_{z x}$ are dissimilar. Note that both equation 5.1.12b and equation 5.1.11 yield the isotropic shear modulus (as expected) when the isotropic case is introduced, with $E_{z}=E_{x}=E$ and $v_{x y}=v_{z x}=v$.

Table 5.1.1 presents the calibrated (backcalculated) anisotropic elastic constants for APT pass number 5,000. The global error term (equation 4.2.7) was $4.73 \%$ which is only slightly lower compared to $4.89 \%$ in the isotropic case (note that similar values of $\min \left(E R R_{g}\right)$ were used to make this comparison valid). Also listed in the table are the Poisson's ratios and the resulting shear moduli calculated according to equation 5.1.11.

Table 5.1.1: Backcalculated anisotropic layer moduli for pass \#5,000.

\begin{tabular}{|c|c|c|c|c|c|c|}
\hline \multirow{2}{*}{$\#$} & \multirow{2}{*}{ Layer } & \multirow{2}{*}{$\begin{array}{c}\text { Thickness, } \\
\text { in. (mm) }\end{array}$} & $\begin{array}{c}\text { Poisson's } \\
\text { Ratio } \\
v_{z x}=v_{x y}\end{array}$ & \multicolumn{2}{|c|}{$\begin{array}{c}\text { Backcalculated Moduli, } \\
\text { psi (MPa) }\end{array}$} & $\begin{array}{c}\text { Shear } \\
\text { Modulus } G_{x z} \\
\text { (equation 5.1.11) } \\
\text { psi (MPa) }\end{array}$ \\
\hline 1 & HMA & $5(127)$ & 0.30 & $152,000(1,050)$ & $358,500(2,470)$ & $75,100(520)$ \\
\hline 2 & Base & $6(152)$ & 0.35 & $61,300(422)$ & $10,850(75)$ & $8,340(58)$ \\
\hline 3 & Subgrade & $61(1,549)$ & 0.40 & $11,950(82)$ & $9,250(64)$ & $3,865(27)$ \\
\hline 4 & Concrete & $\begin{array}{c}\text { Semi- } \\
\text { infinite }\end{array}$ & 0.20 & $4,000,000(27,580)$ & $\begin{array}{c}|c| 666,670 \\
(11,490)\end{array}$ \\
\hline
\end{tabular}

It may be seen that both the subgrade and aggregate base were found to be stiffer in the vertical direction compared to the horizontal direction. Because compaction processes produce preferred aggregate orientation (e.g., Oda et al., 1985; Saadeh et al., 2002) and lock-in of horizontal stresses within the different layers (e.g., Uzan 1985; Duncan et al. 1991), this outcome is expected (at least conceptually). For the HMA, however, the trend is reversed with greater stiffness in the horizontal direction. Quantitatively, the ratio of $E_{z} / E_{x}$ is $1.29,5.65$ and 0.42 for the subgrade, base and HMA (respectively). 
In the vertical $(z)$ direction, the HMA is only 2.5 times stiffer than the underlying aggregate base; this ratio seems relatively low. The base itself is about 5.0 times stiffer than the subgrade; a ratio that is relatively high considering the thinness of the layer and structure. In the horizontal $(x-y)$ direction, the HMA was found to be 33 times stiffer than the aggregate base; the value of $E_{x}$ seems too low for the base (only 1.2 stiffer than the subgrade), but reasonable for the HMA.

By comparison with the isotropic case for pass number 5,000 (see Table 4.2.1) it may be seen that the isotropic analysis gives moduli values that more or less range between $E_{z}$ and $E_{x}$ (separately for each layer); the stiffness ratios, however, do not match.

Figure 5.1.3 graphically contrasts the measured and computed responses in the APT (refer to Figure 3.5.1). Gauge readings are shown using solid markers (two types) and the anisotropic model responses are shown using solid lines. The isotropic case, reproduced from Figure 4.2.2, is also included (using dashed lines) for comparison. As can be seen, the anisotropic model offers only a slight advantage in capturing the measured responses compared to the isotropic case. Both models underestimate the peak vertical stresses on top of the base (gauges 1178 and 1187) and the peak horizontal strains in the $\mathrm{X}$ direction measured between the dual-wheel assembly (gauges G1 and G3). The other three responses are relatively well reproduced. 


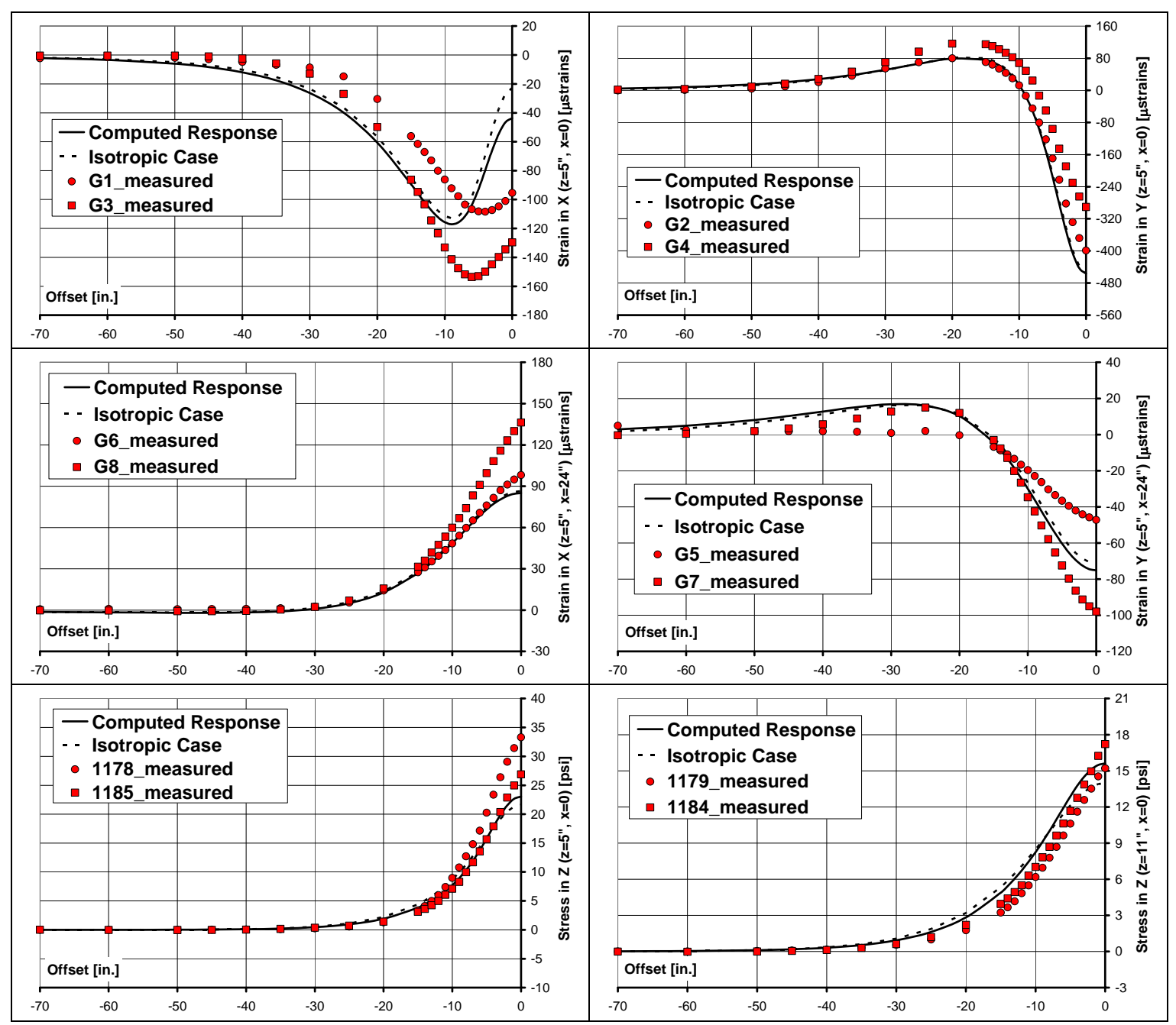

Figure 5.1.3: Comparison of measured resilient responses in the APT during pass \#5,000 with responses computed using the anisotropic layered model (isotropic case is reproduced from Figure 4.2.2).

\subsubsection{NCAT Response Prediction}

In this subsection the anisotropic layered model is applied to forecast resilient responses measured at NCAT. Guided by the methodology developed in Chapter 4, the computations are done with the calibrated properties shown in Table 5.1.1; the HMA moduli are adjusted to account for the differences in loading speed and temperature (relative to the APT experiment). For the anisotropic HMA case, there is an additional assumption that the ratio $E_{z} / E_{x}$ is unaffected by loading speed and temperature (i.e., remains equal to 0.42 ). 
Referring first to peak FWD deflections, the first three basins obtained on Nov. 3, 2003, Dec. 15, 2003, and on Jan. 26, 2004 are considered (Table 2.3.9). In the isotropic analysis of these same deflections (see Subsection 4.3.2), the adjusted HMA moduli were: $\left.E_{\text {HмA }}\right|_{\text {Nov.03 }}=1,000 \mathrm{MPa}(145,000 \mathrm{psi}),\left.E_{\text {HMA }}\right|_{\text {Dec. } 03}=2,900 \mathrm{MPa}(420,500$ psi) and $\left.E_{\text {HMA }}\right|_{\text {Jan.04 }}=4,600 \mathrm{MPa}(667,000 \mathrm{psi})$. These stiffness values reflected (respectively): $41.4 \%, 120.1 \%$ and $190.6 \%$ of the APT backcalculated modulus of 2,412 MPa (350,000 psi; see Table 4.2.1). These percentages were applied to the anisotropic HMA moduli in Table 5.1.1; the resulting stiffnesses are listed in Table 5.1.2.

Table 5.1.2: Adjusted anisotropic HMA moduli for FWD response prediction.

\begin{tabular}{|c|c|c|}
\hline $\begin{array}{c}\text { FWD Test Date } \\
\text { (refer to Table 2.3.9) }\end{array}$ & $\begin{array}{c}\text { Vertical Modulus } E_{z}, \\
\text { psi (MPa) }\end{array}$ & $\begin{array}{c}\text { Horizontal Modulus } E_{x}, \\
\text { psi (MPa) }\end{array}$ \\
\hline Nov. 3, 2003 & $63,000(435)$ & $148,420(1,023)$ \\
\hline Dec. 15, 2003 & $182,550(1,260)$ & $430,560(2,970)$ \\
\hline Jan. 26, 2004 & $289,700(2,000)$ & $683,300(4,711)$ \\
\hline
\end{tabular}

Figure 5.1.4 shows the peak measured FWD deflections at NCAT (solid markers) and also the corresponding computed/projected deflections using the anisotropic LET (solid lines); the isotropic case from Figure 4.3.3 is reproduced here for comparison (dashed lines). It may graphically be seen that the trend in the computations follows the trend in the data. Quantitatively, the average absolute difference between test data and the anisotropic model for the three dates considered is 33.6 microns (vs. 32.4 microns in the isotropic case); the average absolute relative error is $40.1 \%$ (vs. $44.1 \%$ in the isotropic case). If the anisotropic model was directly calibrated using the NCAT deflections, allowing only for the HMA modulus to differ in each case (while maintaining the ratio: $E_{z} / E_{x}=0.42$ ), the aforementioned errors would have been 12.0 microns and $20.1 \%$ respectively (vs. 12.8 microns and $22.2 \%$ in the isotropic analysis). Hence, the forecasting errors of the anisotropic model are 2.8 to 2.0 times higher compared to the calibrated case. This outcome was also obtained in the isotropic case. 
Based on these findings it may be concluded that the anisotropic analysis FWD deflections offers little advantage over an isotropic analysis.

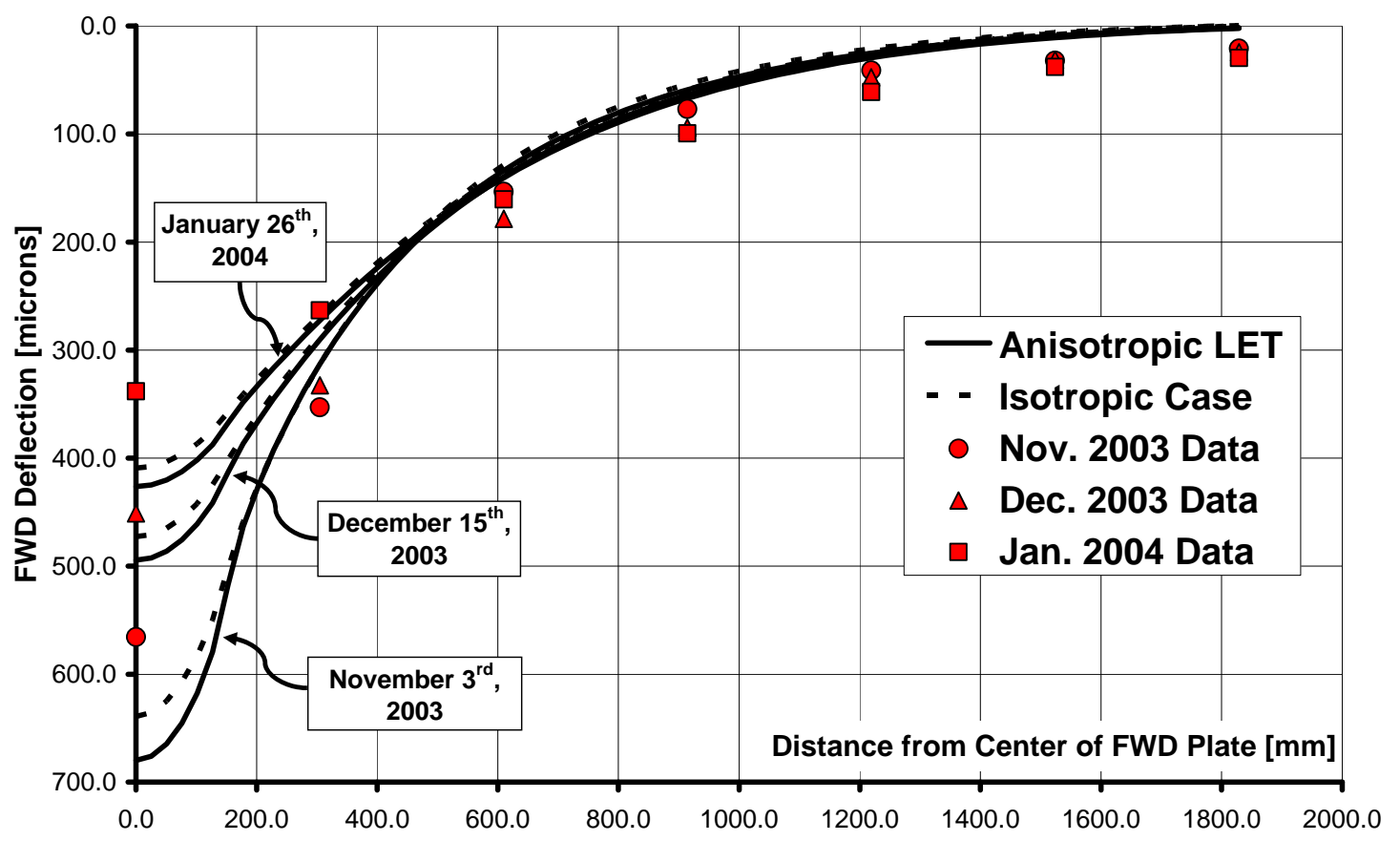

Figure 5.1.4: Comparison of measured peak FWD deflections at NCAT N1 with projected peak deflections using anisotropic LET (isotropic case reproduced from Figure 4.3.3).

Referring next to the projection of truck traffic responses, recall that in the isotropic case the HMA modulus was adjusted from 350,000 psi (2,414 MPa) in the calibrated APT model to 290,000 psi (2,000 MPa) for NCAT conditions (Subsection 4.3.3). This $17.1 \%$ reduction was calculated based on the combined dynamic modulus master curve (Figure 4.3.1) and on the combined time-temperature shifting (Figure 4.3.2) to reflect both truck speeds and HMA temperatures. For the anisotropic case, assuming a constant $E_{z} / E_{x}(=0.42)$, the new HMA moduli for NCAT speed and temperature are simply those in Table 5.1 .1 but each reduced by $17.1 \%$. As before, all other layer properties remain unchanged for the forward calculations.

In the following two figures, the computed and measured NCAT responses are graphically contrasted. Given that the location of the NCAT trucks was not measured, assumptions were made regarding their location relative to the gauge array. First, the travel paths of the truck axles shown in Figure 4.3.5 were reused for performing the 
forward calculations in the anisotropic case. Recall that these were obtained by the requirement that the model matches the measured vertical stress responses (hence the stress responses should not used to assess the modeling and predictive scheme). Second, in order to position the axles longitudinally, the calculated and measured peaks were made (forced) to coincide with each other.

With reference to Figure 2.1.2, Figure 5.1.5 is devoted to the responses caused by the steer axle (1S), and Figure 5.1.6 is devoted to the responses caused by the third trailer axle (3T). As performed in Subsection 4.3.3, each figure is comprised of nine charts, depicting the calculated (solid lines) and measured (circular markers) response of the individual gauges shown in Figure 4.3.5. The abscissa represents time in seconds, matching the timeline in Figures 2.5.1 to 2.5.4. The ordinate depicts either vertical stress (in psi) or horizontal strain (in microstrains) depending on the gauge considered. Note that the scale changes from chart to chart. Each figure also includes a picture of the NCAT truck with an arrow identifying the axle being considered. The isotropic predictions, seen in Figures 4.3.6 and 4.3.9, are reproduced here for graphical reference using dashed lines.

As a general observation, it may be seen in these figures that the solid and dashed lines essentially coincide, indicating that the anisotropic model offers little advantage over the isotropic case. Consequently, for the pavement system herein considered, it seems that the added complexity involved in the anisotropic analysis (although conceptually appealing) did not prove worthy. It very well may be that this outcome transpired from imposing a time-independent model on the test data. Henceforth, in the following section, time-dependence is introduced into the (isotropic) model by treating the HMA, and therefore the entire system response, as viscoelastic. 


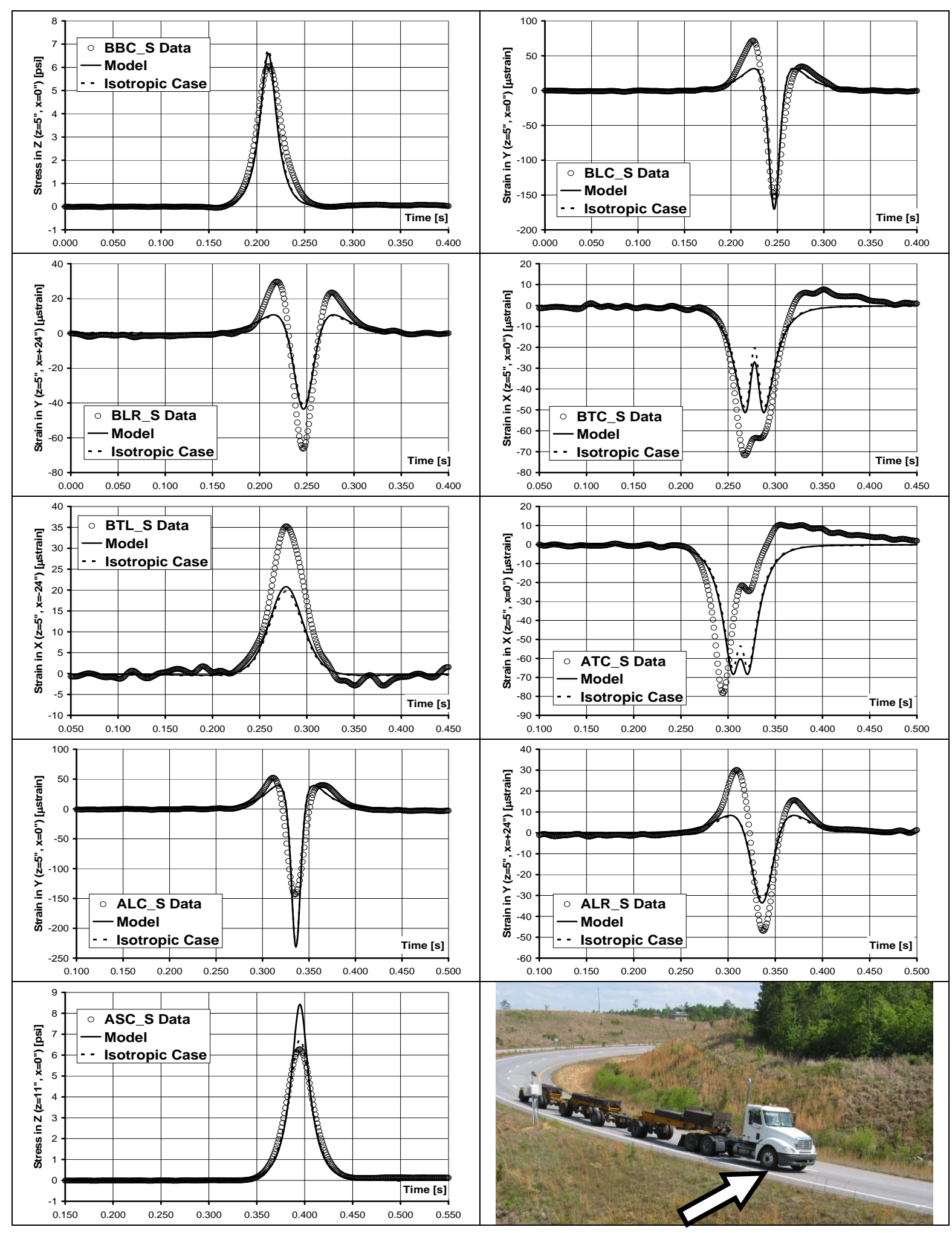

Figure 5.1.5: Comparison of anisotropic LET projections with measured N1 responses right side of steering axle (1S). Isotropic case reproduced from Figure 4.3.6. 


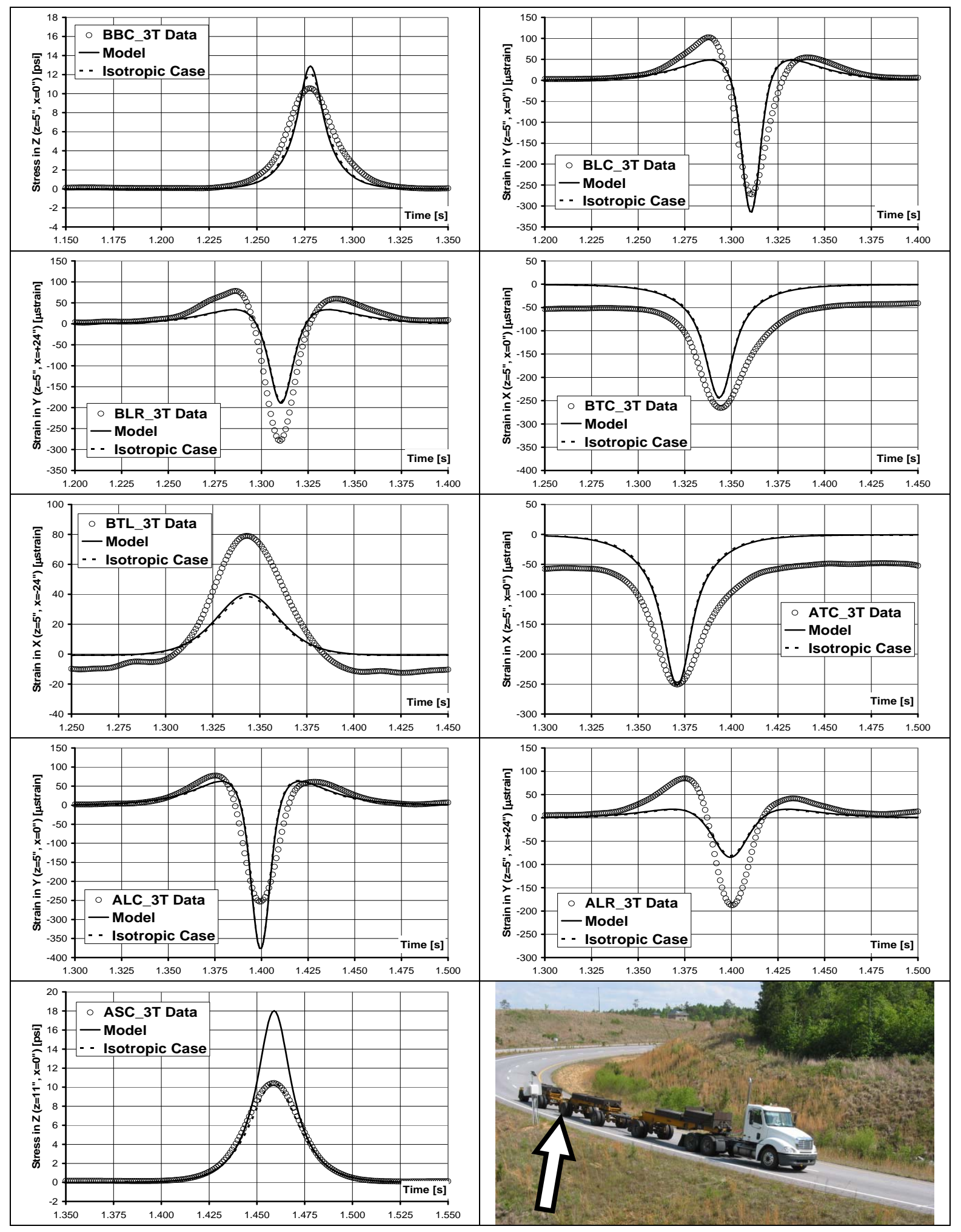

Figure 5.1.6: Comparison of anisotropic LET projections with measured N1 responses right side of third trailer axle (3T). Isotropic case reproduced from Figure 4.3.9. 


\subsection{LAYERED VISCOELASTIC ISOTROPIC MODEL}

\subsubsection{Theory and Computational Implementation}

The time-dependent response $R^{v e}(t)$ of a linear viscoelastic (non-aging) system under isothermal conditions to a given time-dependent input $I(t)$ is fully characterized by a function $R_{H}^{v e}(t)$ named indicial admittance (von Karman and Biot, 1940). This function represents the response of the system to a unit input applied as a step function in time, i.e., $R_{H}^{v e}(t)=R^{v e}(t)$ for the case where $I(t)=H(t)$ in which $H(t)$ is the Heaviside unit step function. In essence $R_{H}^{v e}(t)$ is a function of the problem geometry, boundary conditions and the material properties; it also depends on the preselected input (e.g., force or displacement on the boundary), and on the type of response of interest (e.g., stress, strain or displacement at a point). Whatever the case may be, once the unit response function $R_{H}^{v e}(t)$ is available, the response to an arbitrary time-varying input can be calculated using Boltzmann’s superposition integral (e.g., Schapery, 1974):

$$
R^{v e}(t)=\int_{\tau=0}^{t} R_{H}^{v e}(t-\tau) \cdot d I(\tau)
$$

in which $t$ is physical time and $\tau$ is a time-like integration variable.

In many cases (e.g., Pipkin, 1972; Lockett, 1972) the analysis of a linear viscoelastic system can converted to a mathematically equivalent linear elasticity problem. In general terms, this 'elastic-viscoelastic' correspondence principal states that a Laplace transformed viscoelastic response to a given input can be obtained from the corresponding response in an associated elastic problem having the same geometry, but with the input replaced by its Laplace transform and all elastic material parameters replaced by the s-multiplied Laplace transform of the corresponding viscoelastic material functions. Subsequently, the time-domain (viscoelastic) response is obtained from Laplace transform inversion of the associated elastic solution (Lakes, 1998).

Accordingly, let $R^{e}(t)$ represent the elastic response in the associated elastic problem corresponding to $R^{v e}(t)$ in equation 5.2.1. Then, the elastic response can be expressed as follows: 


$$
R^{e}(t)=R_{H}^{e}\left(c_{1}^{e}, c_{2}^{e}, \ldots\right) \cdot I(t)
$$

in which $I(t)$ is the input and $R_{H}^{e}$ is the indicial admittance of the associated elastic system. The function $R_{H}^{e}$ is seen to depend on a set of elastic time-independent material constants denoted here as $c_{1}^{e}, c_{2}^{e}$, etc, corresponding to a set of viscoelastic (timedependent) material properties: $c_{1}^{v e}, c_{2}^{v e}$, etc. Although not shown explicitly, $R_{H}^{e}$ also depends on the geometry of the problem, boundary conditions and the type of response being calculated.

The indicial admittance of the viscoelastic system $R_{H}^{v e}(t)$ can be obtained by first setting the input $I(t)$ in equation 5.2.2 to equal a unit input $H(t)$ and then applying the elastic-viscoelastic correspondence principal. The resulting expression is:

$$
\bar{R}_{H}^{v e}(s)=R_{H}^{e}\left(s \cdot \bar{C}_{1}^{v e}(s), s \cdot \bar{C}_{2}^{v e}(s), \ldots\right) \cdot(1 / s)
$$

where the macron (or overbar) denotes a Laplace transformed time-function with $s$ being the transform variable. Furthermore, it can be seen that the elastic constants were replaced by the s-multiplied Laplace transforms of the corresponding viscoelastic material properties. The term $(1 / s)$ on the right hand side is the Laplace transform of the unit load $H(t)$. By defining the Carson transform as the s-multiplied Laplace transform (denoted by an overtilde), equation 5.2 .3 can be rearranged as follows:

$$
\widetilde{R}_{H}^{v e}(s)=R_{H}^{e}\left(\tilde{c}_{1}^{v e}(s), \widetilde{c}_{2}^{v e}(s), \ldots\right) .
$$

At this point, the correspondence principal requires performing a Laplace transform inversion of $\tilde{R}_{H}^{v e}(s)$ to obtain $R_{H}^{v e}(t)$. This last step is most often impossible to perform using exact methods because either the elastic solution in known only numerically or its analytic form cannot be inverted; the use of realistic relaxation and creep functions adds further complexity to analytical inversion methods. In view of these complications, Schapery (1962) had proposed two transform inversion methods for which only the numerical values of the elastic solutions are required. The first is the so-called 'collocation method' in which the elastic solution is first collocated by a finite Dirichlet (or Prony) series and later inverted analytically. In principle, increasing the 
length of the series increases the accuracy of the method, but at the cost of additional computation time. The second inversion method is known as the 'direct method'. It is much easier and faster to apply, and although it offers little means of reducing error, several studies have found it adequate for practical (engineering) purposes (Schapery, 1965; Hufferd and Lai, 1978). The 'direct' method was selected for use herein, and is briefly described in what follows.

The Carson transform of $f(t)$ is defined as:

$$
\tilde{f}(s)=s \cdot \int_{t=0}^{+\infty} f(t) \cdot e^{-s t} \cdot d t
$$

Using the change of variable $w=\log (s \cdot t)$, equation 5.2 .5 becomes:

$$
\tilde{f}(s)=\int_{-\infty}^{+\infty} f\left(\frac{10^{w}}{s}\right) \cdot g(w) \cdot d w
$$

In which $g(w)=10^{w} \cdot e^{-10^{w}} \cdot \ln (10)$. The key point of the 'direct inversion' method lies in the properties of the function $g(w)$ which is quasi-null everywhere except for $-2 \leq w \leq 1$ and also satisfies the relation:

$$
\int_{-\infty}^{+\infty} g(w) \cdot d w=1
$$

Therefore, $g(w)$ may be replaced in equation 5.2.6 by a Dirac delta function of the form: $\delta\left(w-w_{0}\right)$. This approximation leads to the direct inversion formula:

$$
\tilde{f}(s) \approx f(\beta / s)
$$

or inversely

$$
f(t) \approx \tilde{f}(\beta / t)
$$

in which $\beta=10^{w_{0}}$. The free parameter $\beta$ (or equivalently $w_{0}$ ) needs to be determined to optimize the approximation accuracy. In the case where $f(t)$ is a power law in time, $\beta$ can be selected to yield the exact inversion. Schapery (1962; 1965; 1974) proposed using $\beta \approx 0.5$. 
Without assuming the numerical value of $\beta$, equation $5.2 .8 \mathrm{~b}$ is first applied to equation 5.2.4, giving:

$$
R_{H}^{v e}(t) \approx \widetilde{R}_{H}^{v e}(\beta / t)=R_{H}^{e}\left(\widetilde{c}_{1}^{v e}(\beta / t), \widetilde{c}_{2}^{v e}(\beta / t), \ldots\right)
$$

After applying equation 5.2.8b again, this time to the right hand side of equation 5.2.9, the parameter $\beta$ cancels out, resulting in:

$$
R_{H}^{v e}(t) \approx R_{H}^{e}\left(c_{1}^{v e}(t), c_{2}^{v e}(t), \ldots\right)
$$

The meaning of equation 5.2.10 is that the indicial admittance of the viscoelastic system $R_{H}^{v e}(t)$ can be approximated at any given time $t$ using the unit response of the associated elastic problem $R_{H}^{e}$ with viscoelastic material functions replacing the corresponding elastic constants and evaluated at time $t$. This procedure is also referred to as the ‘quasi-elastic’ approximation (Schapery, 1962; 1965; 1974).

It is important to point out that, due to the assumption of linearity, equation 5.2.1 along with the entire aforementioned derivation can be generalized to compute the response due to several $\alpha$ inputs $(\alpha=1,2, \ldots)$ :

$$
R^{v e}(t)=\sum_{\alpha} \int_{\tau=0}^{t} R_{H \alpha}^{v e}(t-\tau) \cdot d I_{\alpha}(\tau)
$$

in which $R_{H \alpha}^{v e}$ is the viscoelastic response $R^{v e}(t)$ due to a unit input $I_{\alpha}(t)=H(t)$, with all other inputs zero (i.e., the viscoelastic indicial admittance for an isolated $\alpha$ input).

Equation 5.2.11 was ultimately used here to calculate preselected responses (e.g., horizontal strains, vertical stresses) due to a moving half-axle at a given speed and under constant temperature conditions. For computational implementation of the above derivation, the isotropic LET program ELLEA1 was applied for generating solutions to the associated elastic problem. Only the HMA modulus was assumed to be timedependent, while the moduli of all other layers and the Poisson's ratio of all layers (including that of the HMA) were assumed to be time-independent. Axle movements ware simulated by sequentially loading and unloading the pavement surface at different points located along the line of travel. A total of 63 points were used in equation 5.2.11 
(i.e., $\alpha=1,2, \ldots, 63$ ) to yield 63 unit response time functions $R_{H \alpha}^{v e}(t)$ at different offset distances. These offsets ranged from -76 in. $(1.93 \mathrm{~m})$ to $+76 \mathrm{in}$. $(1.93 \mathrm{~m})$ relative to the evaluation point, with 31 points before (approaching) the evaluation point, 31 points after the evaluation point, and one additional point exactly in line with the evaluation point. Spacing of these points ranged between 4 in. $(101.6 \mathrm{~mm})$ to $1.0 \mathrm{in}$. $(25.4 \mathrm{~mm})$ with denser spacing closer to the evaluation point.

A triangular loading shape was applied at each of the loading points. This was done in such a way that the pavement system always carried the full load of the halfaxle. As a point on the pavement was loaded, the previous (adjacent) point was unloaded. When the peak load was reached at a given loading point, the load was completely removed from the previous loading point that same instant. This scheme is demonstrated in Figure 5.2.1. The lower part of the figure shows one evaluation point (crossed circle) and also six load application points (out of the 63) located along the Yaxis (refer also to Figure 3.5.1). The spacing of these points is denoted by $\Delta y_{\alpha}$, with the subscript indicating that the spacing was not uniform.
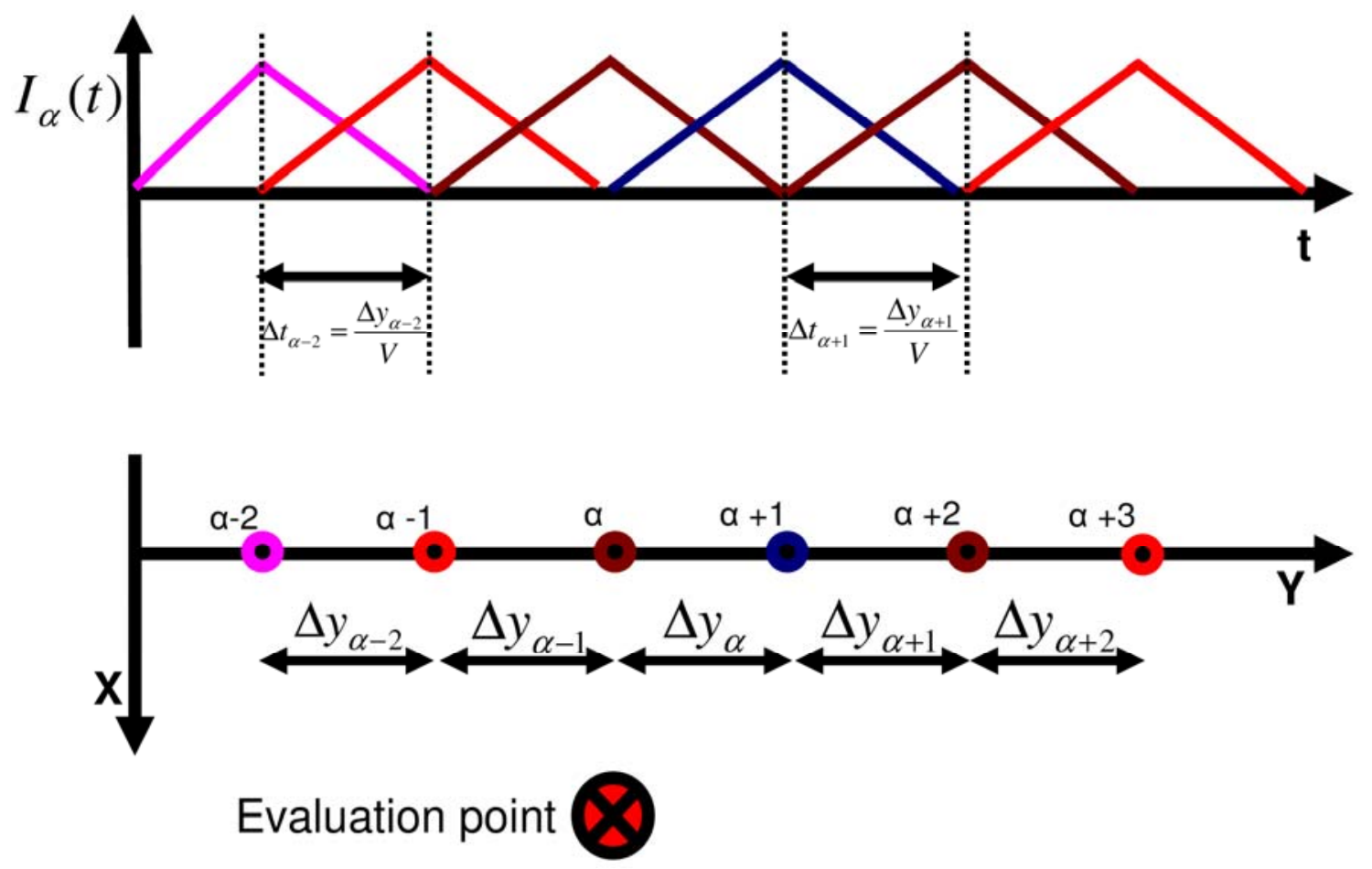

Figure 5.2.1: Scheme for simulating a moving load on a layered viscoelastic model. 
The upper part of Figure 5.2.1 shows the individual 'input' functions vs. time, illustrating the triangular load-unload shape applied to each point. Given that the loading points were not spaced uniformly apart, the time difference between adjacent load peaks (denoted as $\Delta t$ ) was varied such that the load would appear to be moving at a constant speed of choice (denoted as $V$ in the figure).

Given a set of time-independent material properties, and a loading point $\alpha$, $R_{H \alpha}^{v e}(t)$ in equation 5.2.11 was first evaluated using ELLEA1. For this purpose, the response of interest due to a unit stress intensity was computed 51 times, each with a different HMA modulus level ranging between an upper bound $E_{0}$ and a lower bound $E_{\infty}$. The time $t$ associated with each modulus level depended on the shape of the relaxation modulus $E(t)$, was which was represented using the expression:

$$
E(t)=\frac{E_{\infty} \cdot\left[1+\left(t / \tau_{D}\right)^{n_{D}}\right]}{\left(t / \tau_{D}\right)^{n_{D}}+\left(E_{\infty} / E_{0}\right)}
$$

in which $\tau_{D}$ (units of time) and $n_{D}$ (unitless) are constants that control the shape that $E(t)$ takes in the 'transition' between the two extreme values $E_{0}$ and $E_{\infty}$ (note that $\lim _{t \rightarrow 0} E(t)=E_{0}$ and also that $\left.\lim _{t \rightarrow \infty} E(t)=E_{\infty}\right)$.

The resulting elastic response, plotted vs. time, formed points on the $R_{H \alpha}^{v e}(t)$ curves. These points were thereafter interpolated using piecewise linear functions (with time in logarithmic scale) for generating $R_{H \alpha}^{v e}(t)$ values for any given $\alpha$ and for any $t$ of choice. As an example, Figure 5.2.2 shows four such curves, each representing the strain response in the travel direction at the bottom of the asphalt layer due to the APT dual-wheel loading with an applied stress level of 1 psi (i.e., unit intensity input). Each curve corresponds to a different offset distance from the evaluation point (indicated in the chart). The strain responses are denoted using solid lines with values depicted on the left ordinate. Values of $E(t)$ are shown on the right ordinate, and time is depicted on the abscissa. In generating these curves, layer properties were taken from Table 4.2.1 (pass number 5,000 data). The HMA modulus was varied between $E_{0}=40825 \mathrm{MPa}$ (5,920,000 psi) and $E_{\infty}=164 \mathrm{MPa}(23,800 \mathrm{psi})$, such that the associated times will be 
evenly spaced on a logarithmic scale. Both extreme modulus values were obtained from the combined dynamic modulus master curve in Figure 4.3.1 using $E_{0}=\lim _{f_{r} \rightarrow \infty}\left|E_{c o m}^{*}\right|$ and $E_{\infty}=\lim _{f_{r} \rightarrow 0}\left|E_{c o m}^{*}\right|$. The constants $\tau_{D}$ and $n_{D}$ were arbitrarily determined as: $\tau_{D}=35,000$ seconds and $n_{D}=0.240$.

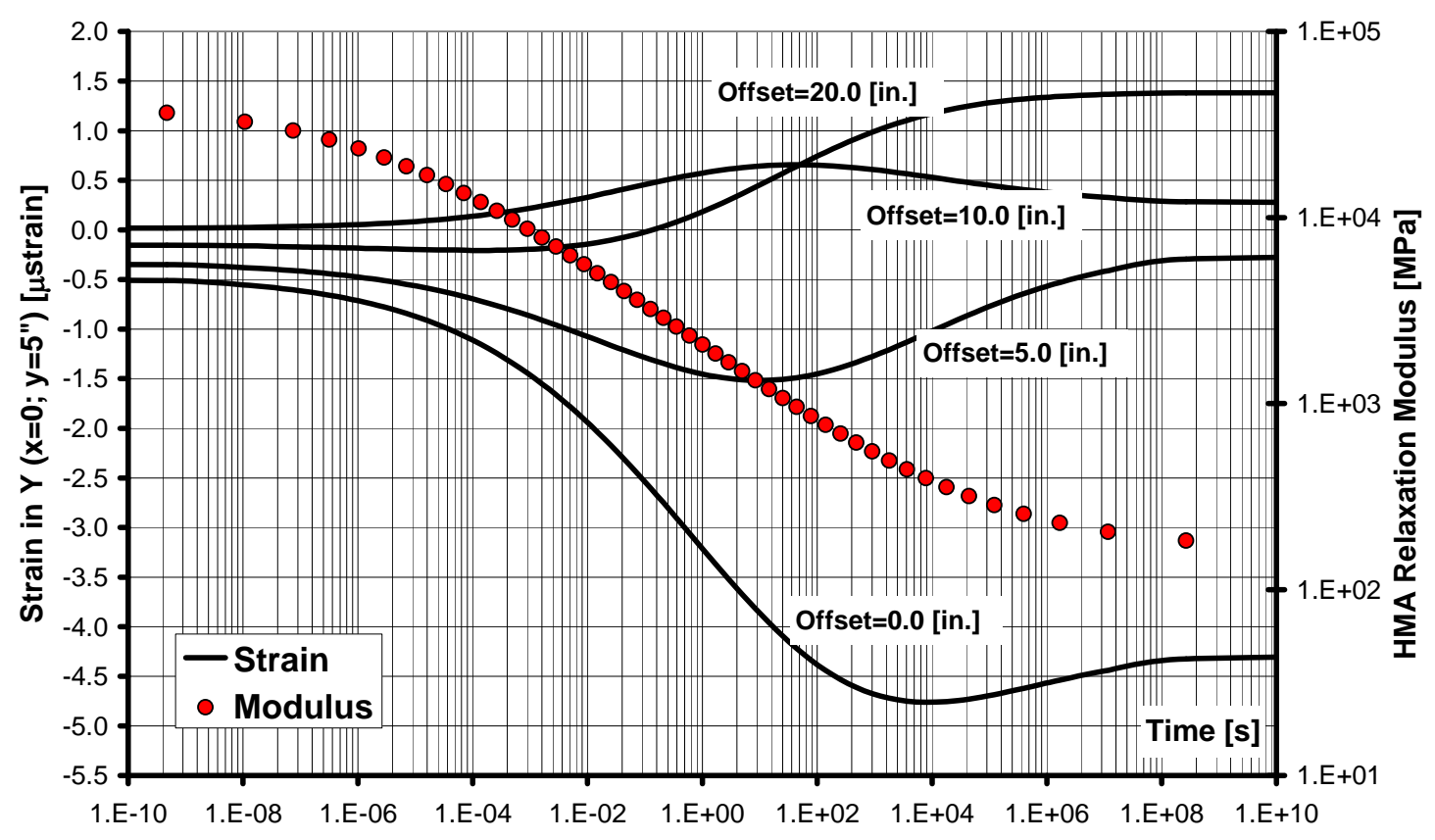

Figure 5.2.2: Indicial admittance of a layered viscoelastic system (example). Strain response due to a unit intensity 'input' of an APT half-axle passing along four offset distances from the evaluation point.

Subsequently, the integration in equation 5.2.11 was commenced; this was performed numerically based on the expression:

$$
R^{v e}\left(t_{n}\right)=\sum_{\alpha}\left(\sum_{m} H\left(t_{n}-\tau_{m}\right) \cdot R_{H \alpha}^{v e}\left(t_{n}-\tau_{m}\right) \cdot \Delta I_{\alpha}\left(\tau_{m}\right)\right)
$$

in which $R^{v e}\left(t_{n}\right)$ is the viscoelastic response of interest evaluated at time $t_{n}, H(\cdot)$ is the unit Heaviside function, $R_{H \alpha}^{v e}\left(t_{n}-\tau_{m}\right)$ is the indicial admittance of the viscoelastic system for a given $\alpha$, evaluated at time $t_{n}-\tau_{m}$, and $\Delta I_{\alpha}\left(\tau_{m}\right)=I_{\alpha}\left(\tau_{m}\right)-I_{\alpha}\left(\tau_{m-1}\right)$. The parameter $n$ ranged between 1 and 23 resulting in 23 values of $t_{n}$, chosen such that the 
shape of the viscoelastic response was adequately captured within the offset range of \pm 70 in. ( $\pm 1.78 \mathrm{~m}$ ). Physically, the calculation points were spaced 2 to 5 in. (50.8 to 127 $\mathrm{mm}$ ) apart; a cubic spline interpolation scheme was used to generate intermediate responses. The parameter $m$ ranged between 1 and 3,200 with values of $\tau_{m}$ chosen such that each triangular load-unload 'input' (see Figure 5.2.1) was divided into 100 time intervals: 50 during loading and 50 during unloading.

It should be noted that the time $t$ associated with each relaxation modulus level $E(t)$ depends also on the temperature considered in the analysis. Using the timetemperature superposition concept it was possible to include temperature dependence in the aforementioned formulation by horizontal shifting of the $E(t)$ curve in Figure 5.2.2. Mathematically, this was accomplished by multiplying $\tau_{D}$ in equation 5.2 .12 by the time-temperature shift factor $a_{T}$ (see equation 3.3.6). Naturally, for analysis performed under the reference temperature in which $E(t)$ was determined, $a_{T}$ equaled unity and equation 5.2.12 was unaffected. For an analysis temperature that was different than the reference temperature, $a_{T}$ departed from unity (see Figure 4.3.2), resulting in horizontal shifting of the $E(t)$ curve. For example, when the analysis temperature was higher than the reference temperature, $a_{T}$ became greater than unity, resulting in horizontal shifting of $E(t)$ to the left. As expected, under such circumstances the material appears softer because the transition from $E_{0}$ to $E_{\infty}$ becomes, in effect, faster.

\subsubsection{Calibration to APT Conditions}

The layered viscoelastic model was calibrated using the time history of the responses measured in the APT during pass number 5,000. Similar to the previous analyses (subsections 4.2.2 and 5.1.2) material properties were determined using a nonlinear error minimization algorithm by matching computed and measured responses as best as possible. The objective function had similar structure to that used previously (see equations 4.2.6 and 4.2.7) so as to ensure that all gauge readings were equally weighted in the backcalculation process. Ideally, the moving half-axle load in the APT should have been applied many times over in the simulation, and the matching performed using 
the responses computed in the last simulated pass. However, due to computational power limitations, only one movement of the half-axle was simulated. Furthermore, it should be noted that unlike the previous analyses (refer to Figures 4.2.2 and 5.1.3), the matching of computed and measured responses was not limited to the approaching branches and was also performed for the receding branches.

The pavement system was modeled as a four layered half-space. Only the top layer, representing the HMA, with a thickness of 5 in. $(127 \mathrm{~mm})$ was treated as viscoelastic while the remaining three layers were treated as time-independent (elastic). A constant Poisson's ratio of 0.30 was assumed for the HMA with a relaxation modulus $E(t)$ that follows equation 5.2.12. The extreme values of $E(t)$ at $t \rightarrow 0$ and $t \rightarrow \infty$ were prefixed to: 5,920,000 psi (40,825 MPa) and 23,800 psi (164 MPa) respectively. These values were obtained from the combined dynamic modulus master curve in Figure 4.3.1 using $E_{0}=\lim _{f_{r} \rightarrow \infty}\left|E_{c o m}^{*}\right|$ and $E_{\infty}=\lim _{f_{r} \rightarrow 0}\left|E_{c o m}^{*}\right|$. The second layer from the top represented the aggregate base with a thickness of 6 in. (152 mm), Poisson's ratio of 0.35 and modulus $E_{2}$. The third layer from the top represented the subgrade with a thickness of $61 \mathrm{in.}(1,549 \mathrm{~mm})$, Poisson's ratio of 0.40 and modulus $E_{3}$. The bottom (fourth) layer with semi-infinite thickness represented the concrete floor of the test pit, having the following properties: $v_{4}=0.20$ and $E_{4}=4,000,000$ psi $(27,580$ $\mathrm{MPa}$ ). Consequently, four unknown parameters were determined by the inverse analysis, namely: the two remaining HMA properties $\tau_{D}$ and $n_{D}$ (equation 5.2.12), the base modulus $E_{2}$, and the subgrade modulus $E_{3}$.

Table 5.2.1 presents the calibrated material properties of the layered viscoelastic model corresponding to APT pass number 5,000. The global error term (equation 4.2.7) was $1.32 \%$ which is much lower than, but not directly comparable to, the timeindependent cases, mainly because matching was performed for both the approaching and receding branches of the responses. As can be seen in the table, the base modulus was found to be lower than the subgrade modulus, which contradicts the findings from the time-independent analyses. With reference to the resilient modulus tests (see Figure 3.3.1), a subgrade modulus of 25,915 psi (180 MPa) seems too high (i.e., exceeding the 
resilient modulus range of test results), and a base modulus of 6,820 psi (47 MPa) appears too low for a material compacted to $97 \%$ (which is the compaction degree in the APT experiment). In comparison with the isotropic LET analysis (Table 4.2.1), the subgrade here is about 2.2 times stiffer; the base modulus here is merely $28 \%$ of that backcalculated in the time-independent isotropic case.

Table 5.2.1: Backcalculated material properties for the layered viscoelastic model during APT pass \#5,000.

\begin{tabular}{|c|c|c|c|c|}
\hline$\#$ & Layer & $\begin{array}{c}\text { Thickness, } \\
\text { in. (mm) }\end{array}$ & $\begin{array}{c}\text { Poisson's } \\
\text { Ratio }\end{array}$ & $\begin{array}{c}\text { Modulus, psi (MPa) } \\
+ \text { equation 5.2.12 parameters }\end{array}$ \\
\hline 1 & HMA & $5(127)$ & 0.30 & $\begin{array}{c}E_{0}=5,920,000(40,825) ; E_{\infty}=23,800(164) ; \\
\tau_{D}=21.8 \mathrm{~s} ; n_{D}=0.532\end{array}$ \\
\hline 2 & Base & $6(152)$ & 0.35 & $6,820(47)$ \\
\hline 3 & Subgrade & $61(1,549)$ & 0.40 & $25,915(180)$ \\
\hline 4 & Concrete & $\begin{array}{c}\text { Semi- } \\
\text { infinite }\end{array}$ & 0.20 & $4,000,000(27,580)$ \\
\hline
\end{tabular}

As for the HMA, Figure 5.2.3 superimposes the backcalculated relaxation modulus $E(t)$, i.e., equation 5.2 .12 and parameters from Table 5.2.1, with the relaxation modulus interconverted from the combined dynamic modulus and phase angle master curves in Figure 4.3.1. The interconversion from the frequency domain to the time was performed using the following equation (Levenberg and Shah, 2008):

$$
E(t)=E_{\infty}+\int_{\tau=0}^{\infty} h(\tau) \cdot e^{-t / \tau} \cdot d(\ln \tau)
$$

in which $h(\tau)$ is the relaxation spectrum given in equation 3.3.7 with parameters listed in Subsection 4.3.1 (calibrated to results in the frequency domain). As can be seen in the figure, although both curves were derived for a reference temperature of $15.5^{\circ} \mathrm{C}$, and although the extreme values $E_{0}$ and $E_{\infty}$ were forced to coincide, the transitions from $E_{0}$ to $E_{\infty}$ are completely different (faster in the backcalculated case). 


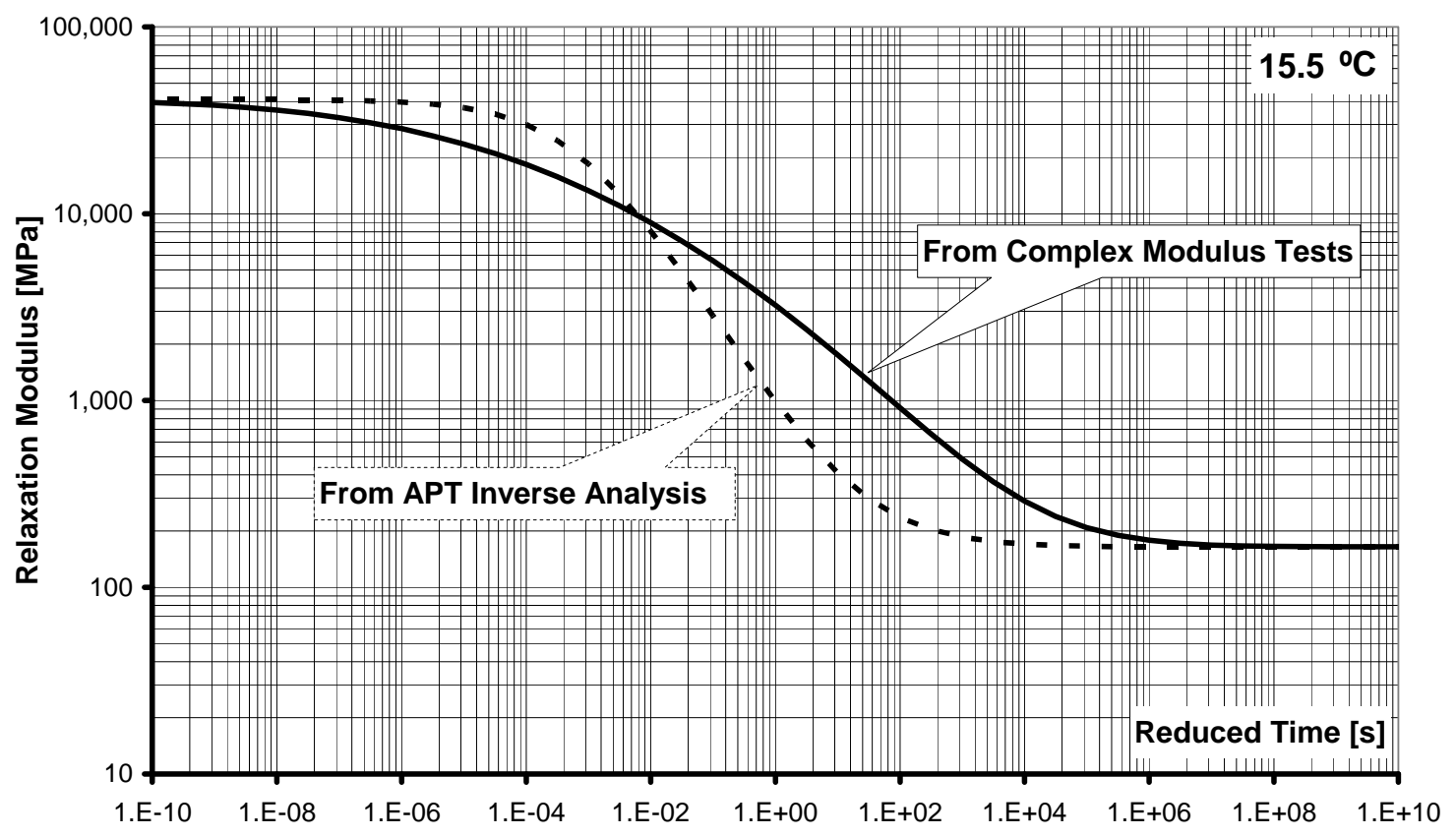

Figure 5.2.3: Comparison of backcalculated relaxation modulus with that interconverted from complex modulus test results.

In the context of inverse analysis, the base and subgrade moduli values, together with the viscoelastic HMA properties, provided the best overall match with the embedded gauge readings; as such they are expected to ensure good forecastability. The apparent unrealistic base and subgrade moduli values, and the differences observed between the relaxation modulus curves in Figure 5.2.3, can be mainly caused by: (i) use of linear elastic isotropic properties to represent the base and subgrade layers and isotropic properties to represent the HMA course; and (ii) simulating just a single load pass to match a response measured after many consecutive load passes. In future studies the first cause can be addressed by using anisotropic elastic (linear or nonlinear) properties to represent the base and subgrade materials and by treating the HMA as anisotropic (viscoelastic); the second cause can be addressed in future studies by simulating more load passes over the viscoelastic system while performing the inverse analysis.

In addition, it should be noted that the dissimilarity in the relaxation modulus curves in Figure 5.2.3 can also reflect differences in strain levels, given that complex modulus tests were performed under very small strains ( 100 microstrains) while the 
backcalculated viscoelastic properties are associated with strain levels that are about four times higher. Furthermore, the dissimilarity can originate from the differences in aggregate structure between an HMA prepared in the laboratory versus an HMA prepared using full-scale construction equipment.

Figure 5.2.4 shows the measured and calibrated model responses for APT pass number 5,000. Six charts are included, each showing a different response vs. offset distance from the gauge. With reference to Figure 3.5.1, the two topmost charts show horizontal strains in $\mathrm{X}$ (left) and in $\mathrm{Y}$ (right) for gauges located along the loading centerline (between the dual tires). The charts in the middle of the figure show horizontal strains in X (left) and in Y (right) for gauges positioned outside the loading path. The bottom charts show vertical stresses as measured by pressure cells located on top of the base (left) and on top of the subgrade (right). In each chart the measured gauge data is represented by two types of solid markers and the calibrated model responses are shown using solid lines.

From the figure it may be graphically seen that the isotropic LVT captures relatively well both the shape and magnitude of all measured responses except for the vertical stresses on top of the base (gauges 1178 and 1185) which are under predicted. It should be noted that the non-symmetry is the responses were also captured very well; the LVT was able to simulate the differences between the approaching and receding response branches and the delay in the peaks (occurring slightly after the load had passed a gauge). 


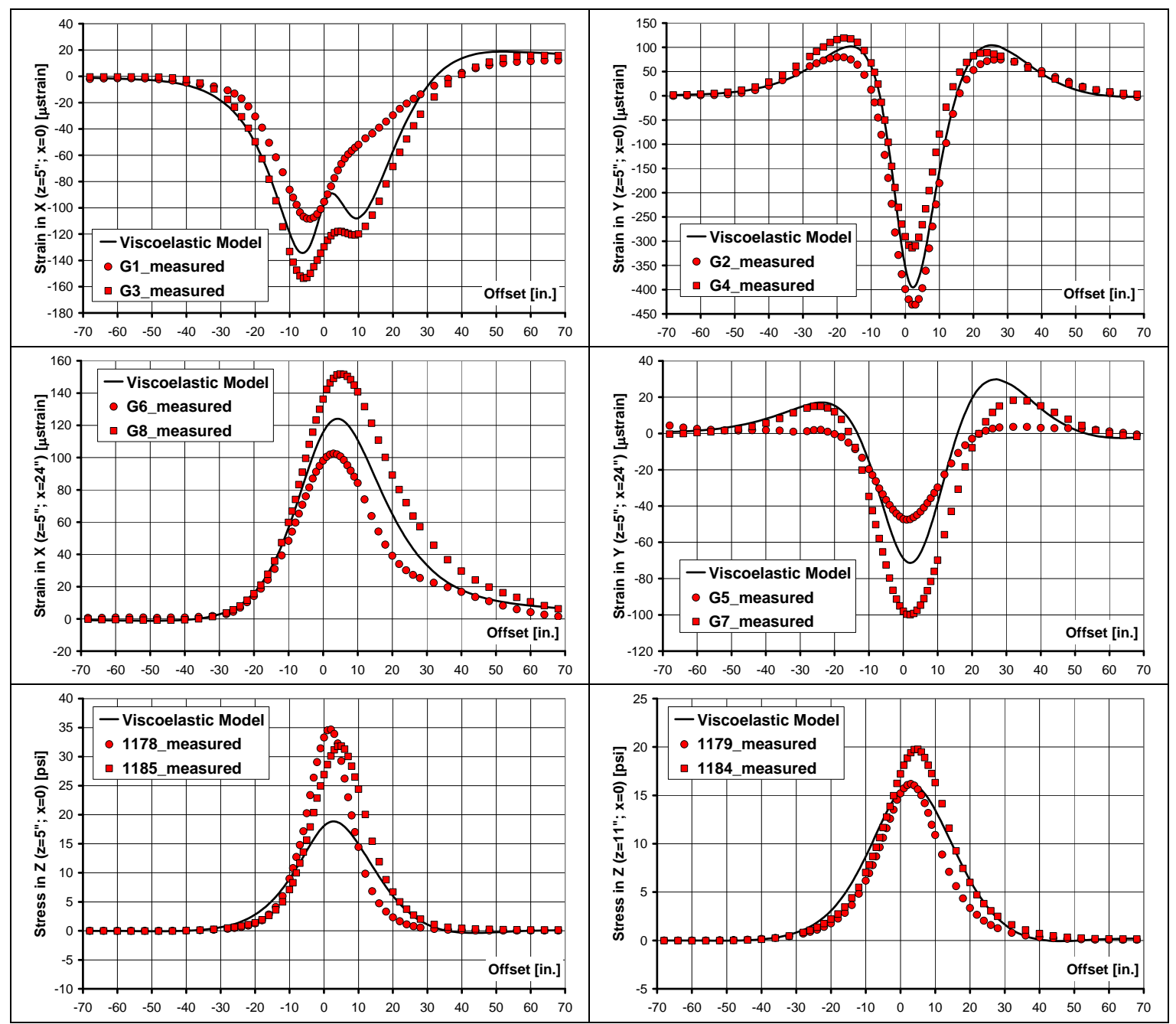

Figure 5.2.4: Comparison of measured resilient responses in the APT during pass \#5,000 with responses computed using the isotropic viscoelastic layered model.

\subsubsection{NCAT Response Prediction}

The calibrated APT model (Table 5.2.1) was used to forecast the NCAT responses due to a moving truck. No attempt was made to forecast peak FWD deflections as was done using the time-independent models, mainly because the time history of the FWD loading is unavailable. Therefore, the forecasting here is aimed at projecting the collected responses shown in Figures 2.5.1 to 2.5.4 as measured by the gauge array in Figure 2.4.3. The fact that layered viscoelastic theory is employed means that all main differences between the APT experiment and NCAT (i.e., axle configuration, axle weight, loading speed and HMA temperature) are endogenously and naturally taken into account. 
As before, the elastic properties of the base and subgrade (and rigid bottom) were assumed to be identical in both experiments, unaffected by the different loading conditions. The appropriate HMA temperature at NCAT was determined in Subsection 4.3.3 to be $80.6^{\circ} \mathrm{F}\left(27.0^{\circ} \mathrm{C}\right)$; based on Figure 4.3.2 this temperature level is associated with a time temperature shift factor of 0.062 (i.e., $a_{T}=0.062$ ). Using a loading speed ( $V$ in Figure 5.2.1) that is nine times higher than the APT, responses were generated by the calibrated viscoelastic model for the different axle configurations and loads given by Table 2.1.1. The travel paths of the truck axles shown in Figure 4.3.5 were reused for performing the forward calculations here. Recall that these were obtained by forcing the measured and calculated stresses to match in the isotropic LET case (see subsections 4.3.3 and 5.1.3). Hence, the modeling capabilities should only be judged based on the strain responses. Additionally, in order to position the axles longitudinally (as their location was not measured in NCAT), the calculated and measured peaks were made (forced) to coincide with each other.

Figures 5.2.5 to 5.2.9 graphically contrast the computational model and the measured resilient responses at NCAT (vs. time). Each figure separately presents the stresses and strains due to a different half-axle. Referring to Figure 2.1.2 and Table 2.1.1, these are respectively: steering wheel (1S), drive axle (1D and 2D), first trailer axle (1T), third trailer axle (3T), and fifth (last) trailer axle (5T). Each figure is comprised of nine charts, individually depicting the measured response (circular markers) and calculated response of the viscoelastic model (solid lines) for the gauges shown in Figure 4.3.5. The isotropic LET case is also shown for graphical comparison (dashed lines), reproduced from Figures 4.3.6 to 4.3.10. The abscissa represents time in seconds, matching the timeline in Figures 2.5.1 to 2.5.4. The ordinate depicts either vertical stress (in psi) or horizontal strain (in microstrains) depending on the gauge considered (note that the scale changes from case to case). In addition, each figure also includes a picture of the NCAT truck with an arrow identifying the half-axle considered.

As a general observation, these figures show that the model predictions capture relatively well the magnitudes as well as the trends in the measured responses. 
Quantitatively, the matching errors in these figures were almost consistently lower than those in Figures 4.3.6 to 4.3.10. This is shown in Table 5.2.2, which lists the improvement in predictive power (in percent) of the viscoelastic model over the isotropic elastic case (as given in Table 4.3.2). As can be seen, the improvement ranged between $0.9 \%$ to $71.7 \%$ with an overall average of $27.8 \%$; only a single negative (worsening) case was obtained (1S axle, ATC gauge).

Table 5.2.2: Relative improvement in response predictions for the isotropic LVT compared to the isotropic LET case given in Table 4.3.2.

\begin{tabular}{|l|c|c|c|c|c|}
\hline \multirow{2}{*}{ Gauge } & \multicolumn{5}{|c|}{ NCAT truck axle designation from Table 2.1.1 } \\
\cline { 2 - 6 } & 1S & 1D+2D & 1T & 3T & 5T \\
\hline BLC & $39.9 \%$ & $47.6 \%$ & $49.3 \%$ & $46.3 \%$ & $20.5 \%$ \\
\hline BLR & $21.2 \%$ & $9.9 \%$ & $7.5 \%$ & $19.8 \%$ & $21.2 \%$ \\
\hline BTC & $64.6 \%$ & $3.9 \%$ & $6.8 \%$ & $42.7 \%$ & $49.0 \%$ \\
\hline BTL & $26.4 \%$ & $71.7 \%$ & $67.5 \%$ & $27.2 \%$ & $0.9 \%$ \\
\hline ATC & $-28.0 \%$ & $2.6 \%$ & $5.0 \%$ & $41.9 \%$ & $51.4 \%$ \\
\hline ALC & $29.0 \%$ & $42.3 \%$ & $52.0 \%$ & $32.7 \%$ & $41.0 \%$ \\
\hline ALR & $15.5 \%$ & $11.8 \%$ & $5.0 \%$ & $7.8 \%$ & $18.3 \%$ \\
\hline
\end{tabular}




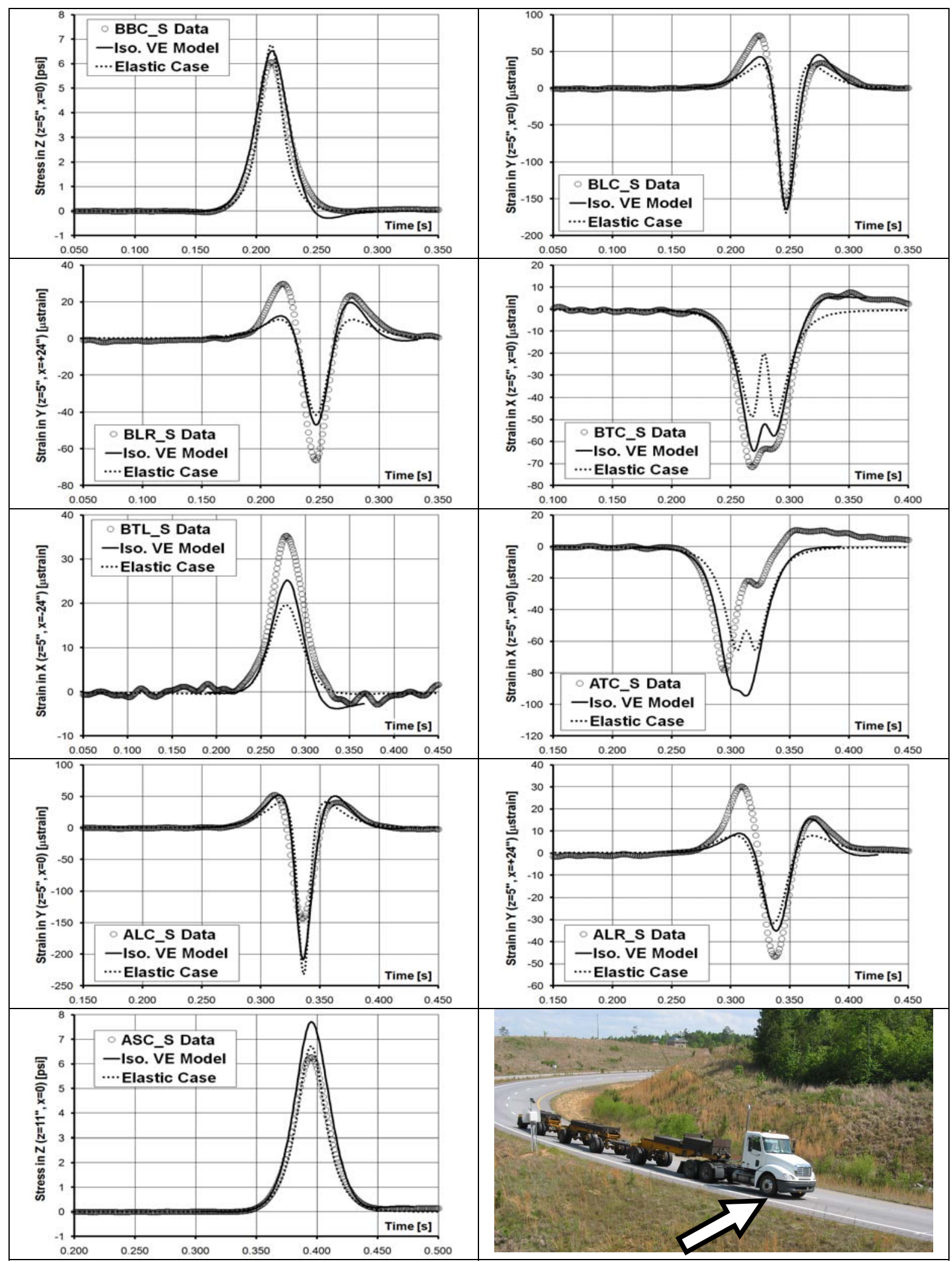

Figure 5.2.5: Comparison of isotropic LVT projections with measured N1 responses right side of steer axle (1S). Isotropic case reproduced from Figure 4.3.6. 


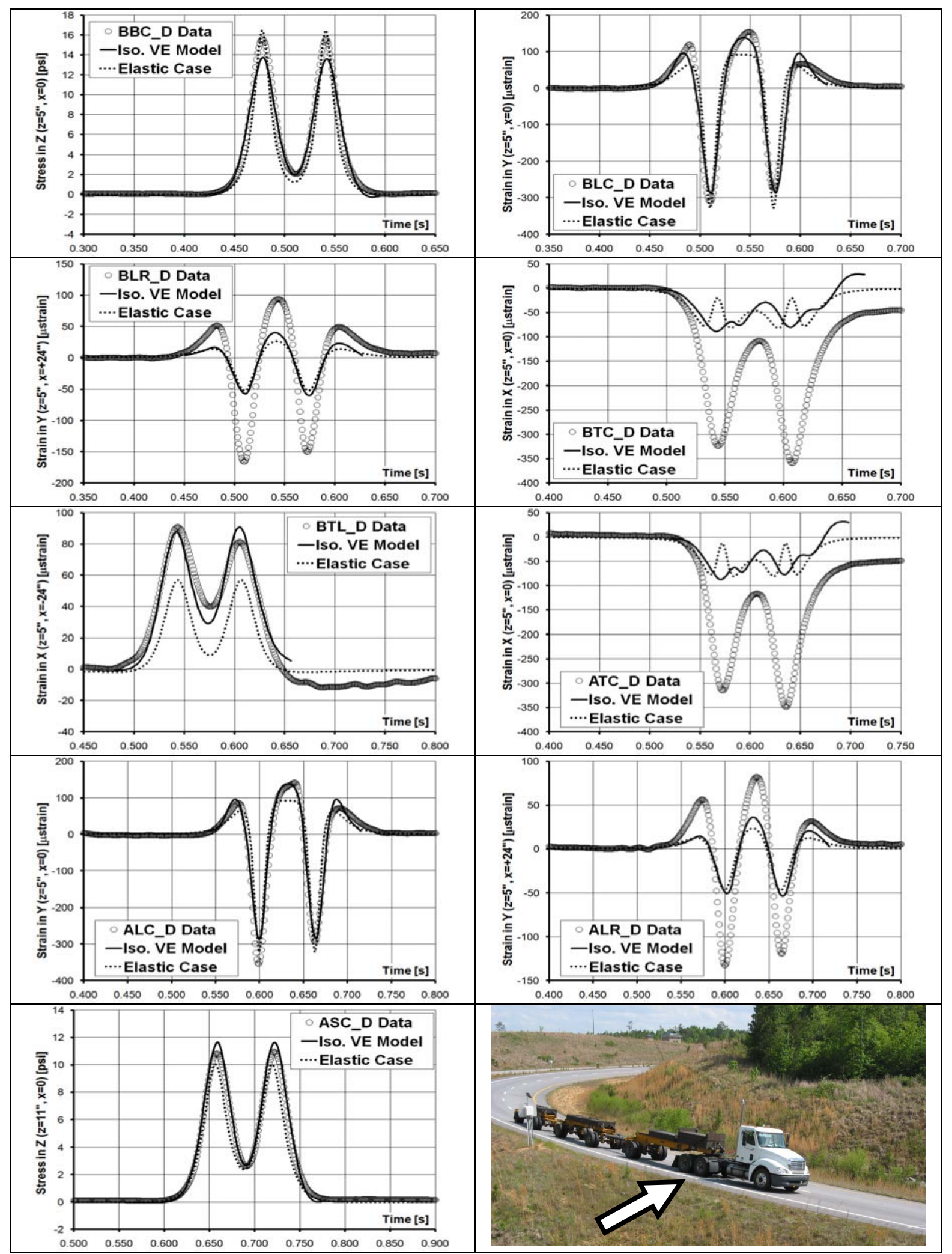

Figure 5.2.6: Comparison of isotropic LVT projections with measured N1 responses right side of drive axle (1D and 2D). Isotropic case reproduced from Figure 4.3.7. 


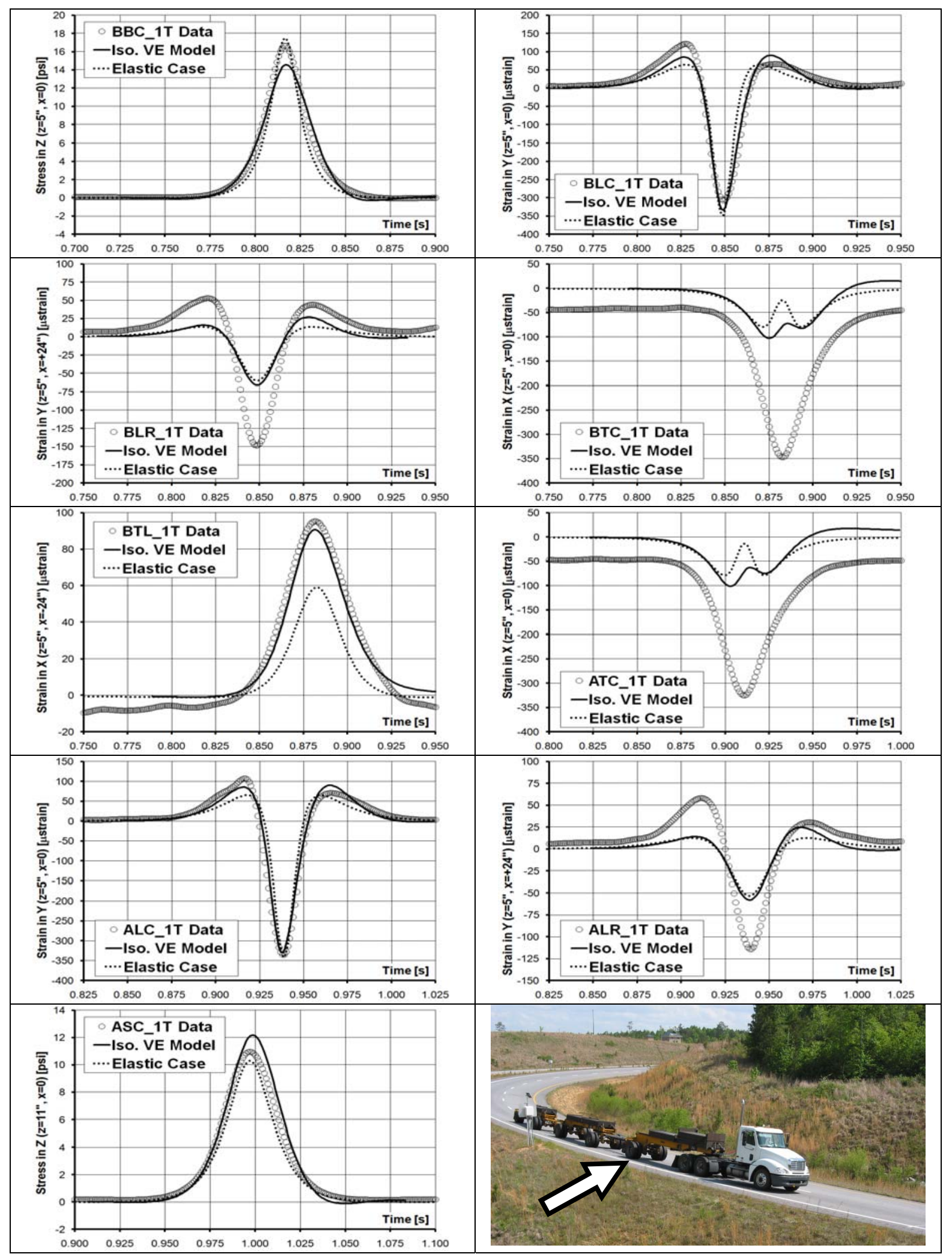

Figure 5.2.7: Comparison of isotropic LVT projections with measured N1 responses right side of first trailer axle (1T). Isotropic case reproduced from Figure 4.3.8. 


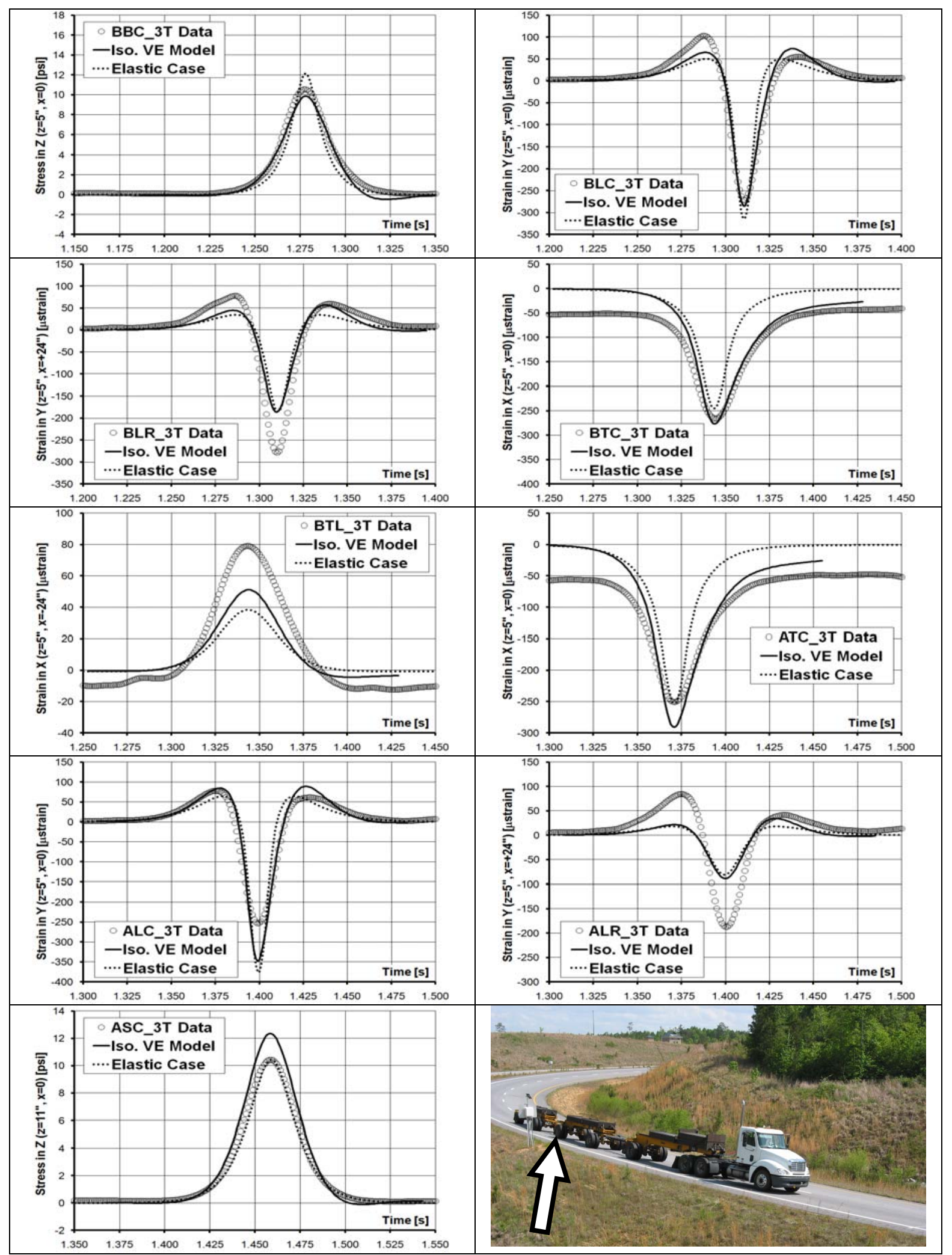

Figure 5.2.8: Comparison of isotropic LVT projections with measured N1 responses right side of third trailer axle (3T). Isotropic case reproduced from Figure 4.3.9. 


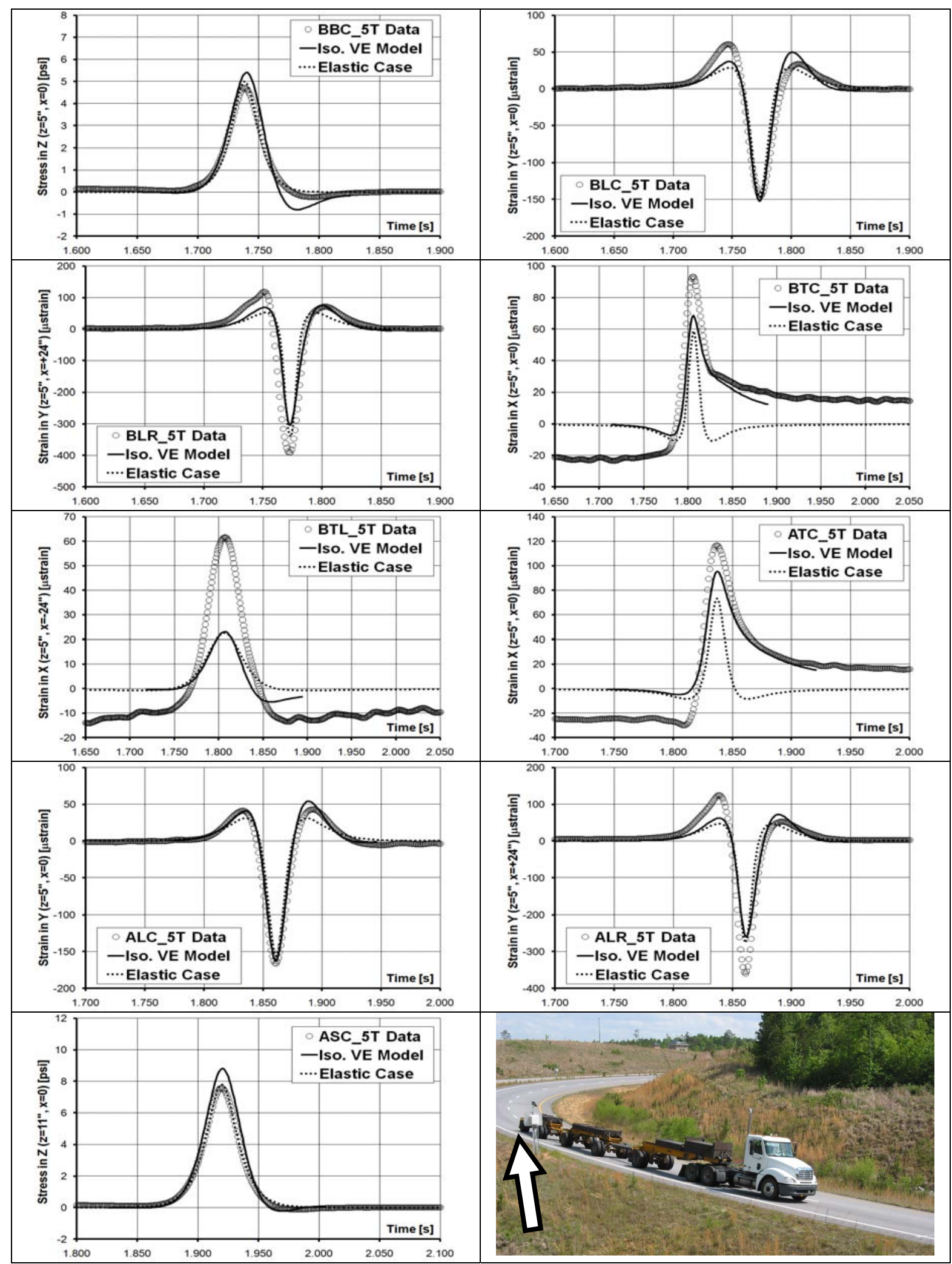

Figure 5.2.9: Comparison of isotropic LVT projections with measured N1 responses right side of fifth (last) trailer axle (5T). Isotropic case reproduced from Figure 4.3.10. 


\subsection{APPRAISAL OF ADVANCED METHODS}

As an extension to Chapter 4, two more advanced pavement models were employed here to address the primary study objective of linking the APT and NCAT experiments, namely: anisotropic layered elasticity, and isotropic layered viscoelasticity. The analyses were performed for the pavements in the very early stages of the experiment, focusing on resilient responses. First, the mathematical derivation of the models was presented in detail. From then on, the models were calibrated using APT response data and later enhanced using laboratory results to apply to other conditions not included in the calibration. Thereafter, the loading and environment at NCAT were simulated and the advanced models applied to forecast measured resilient responses consisting of peak FWD deflections, and stresses and strains induced by a moving truck. The calculated results were graphically and quantitatively compared with the measurements.

Referring first to the anisotropic LET, the model requires five elastic constants for characterizing each layer. For calibration purposes, only two constants were manipulated, namely the elastic moduli in the vertical and horizontal directions; the numerical values of the remaining parameters were assumed. It was found that both the subgrade and base were stiffer in the vertical direction (which can be expected) while the HMA was found to be stiffer in the horizontal direction. While the calibration error was only slightly lower compared to the isotropic case, questionable stiffness ratios and levels were produced. After applying the model to forecast NCAT responses it was found, both graphically and quantitatively, that the anisotropic treatment yielded only slight improvements over the isotropic case. In conclusion, although the application of anisotropic behavior is conceptually appealing, for the pavement system herein considered, the added complexity involved in the anisotropic analysis did not prove worthy.

Referring next to the isotropic LVT, the subgrade and aggregate base were each characterized as time-independent using an elastic modulus and a Poisson's ratio. The HMA was characterized as viscoelastic using a relaxation modulus and a constant Poisson's ratio. A mathematical expression consisting of four parameters was used to represent the relaxation modulus. Two out of the four parameters were directly 
determined from complex modulus test results. The calibration to APT conditions was performed after assuming the numerical values of the Poisson's ratios and manipulating the remaining material parameters. Hence, in effect, the viscoelastic analysis included only one additional free material parameter compared to the basic isotropic layered elastic analysis. The calibration produced seemingly unrealistic unbound material properties with a very high subgrade stiffness and very low base stiffness, much lower than the subgrade. Also, the backcalculated HMA relaxation modulus did not coincide with that obtained from complex modulus tests. The probable reasons for these findings were discussed in the text along with possible corrective measures (yet to be implemented). Nevertheless, when forecastability is considered, the LVT proved superior to the other theories and as such should be preferred in any future attempt to apply APT results to other conditions. 


\section{CHAPTER 6 - CONCLUSION}

This chapter offers a short summary of the entire report and highlights the main findings/results (Section 6.1). In Section 6.2, general recommendations are suggested including future research ideas, followed by specific advice on how INDOT should implement the study results.

\subsection{SUMMARY AND FINDINGS}

The main objective of this study was to devise and validate an analysis scheme by which experimental data collected in INDOT's APT facility could be used to successfully forecast the corresponding pavement behavior at the NCAT test track. More details related to the overall objective and approach can be found in Chapter 1 .

Only one pavement system was addressed, consisting of a relatively thin structure composed of 5 in. $(127 \mathrm{~mm})$ of HMA and 6 in. (152 mm) of aggregate base overlaying an untreated silty soil serving as subgrade. In both the APT and NCAT studies this pavement system was built with embedded instrumentation, in order to measure environmental changes (temperatures, moisture content) and load induced transient responses (vertical stresses, horizontal strains). The pavement was loaded during the Phase II experiment at NCAT between the years 2003 and 2005 while the APT study took place between the years 2004 and 2006. In association with these studies, laboratory tests were also performed on the individual pavement constituents.

The main study objective was pursued in this report according to the following steps: (i) development of mechanistic models to represent the pavement system; (ii) analysis of laboratory test results; (iii) calibration of the necessary material properties from the APT experiment by means of inverse analysis; (iv) enhancement of the modeling capabilities to apply to other loading and environmental conditions not included in the calibration using laboratory test results; (v) simulation of the loading and environmental conditions at NCAT and forward calculation of load induced responses due to an FWD and a moving truck; and (vi) comparison of measured and calculated responses to assess the forecastability of the proposed scheme. 
Chapter 2 included relevant information from the NCAT study. Chapter 3 summarized the APT work and laid the groundwork for commencing the mechanistic analyses. These two chapters revealed that:

(i) Even though nominally identical pavement systems were constructed in the APT and at NCAT, the differences in loading and environmental conditions produced completely distinct responses and dissimilar cracking and rutting performances. Hence, it was concluded that a direct (empirical) relation between the two experiments cannot be established and that it is unavoidable to apply a more fundamental/rational, mechanistic based approach;

(ii) Neither the APT nor NCAT studies were designed and carried out with pure mechanistic interpretation in mind. Partially for this reason, a large part of the collected data in the APT could not be utilized. Similarly, only limited means for validating the proposed scheme were offered by the NCAT data;

(iii) The instrumentation used in both experiments to observe and record mechanical responses was suited for monitoring dynamic transient responses but not for monitoring permanent responses. This fact confined the subsequent analyses to focusing on resilient (recoverable) responses only;

(iv) In the APT study, large differences in response were observed in pairs of gauges that were expected to record identical readings. These differences were assumed to be the result of structural heterogeneity (also manifested in the rutting results) and slight dissimilarities in gauge installation conditions. Because of these differences the advantage of using more sophisticated models to represent the pavement system may not be evident/noticeable;

(v) Due to the controlled conditions in the APT facility it was possible to observe that the resilient responses exhibited permanent changes during the experiment, presumably due to load generated permanent changes in the material properties. Similar findings could not be made for the NCAT study because of the changing environmental conditions and because the axle loadings were not applied exactly at the same location (and the location of application relative to the gauges was not 
recorded). Mainly for this reason, subsequent analyses were focused on the initial stages of both experiments.

Both Chapters 4 and 5 contained the development of mechanistic models for representing the pavement system. These were followed by calibration procedures and utilization of laboratory test results to enhance the applicability of these models to other loading and environmental conditions. Then, the models were used in forward calculation mode to forecast load induced resilient responses at NCAT. Finally, the calculations were compared with the measurements to assess their forecastability.

In Chapter 4 the pavement was modeled as an isotropic layered elastic halfspace (see program ELLEA1 in Appendix B). Four layers were used with properties calibrated under APT conditions using backcalculation by manipulating the elastic constants until model-generated responses matched as closely as possible the time history of all embedded gauge readings collected during one APT pass. This inverse analysis procedure is a key point in the proposed methodology, both for the basic model and for the more advanced models, as it minimizes any systematic errors associated with the modeling simplifications. After calibration, the model was extended to apply to other loading and environmental conditions using complex modulus test results. This was accomplished by varying the stiffness of the HMA to reflect changes in temperature and loading speed relative to the APT conditions while maintaining all other material properties. The extended APT model was then used to forecast load induced responses at NCAT, consisting of peak FWD deflections, and stresses and strains resulting from a moving truck. Considering the relative simplicity of the above scheme and the small number of free parameters used to represent the pavement system, the isotropic layered elastic model performed relatively well in projecting resilient responses at NCAT.

In Chapter 5 the pavement was represented using two more advanced models: anisotropic layered elasticity (see program ELLEA2 in Appendix B), and isotropic layered viscoelasticity. Again (and separately for each case), the models were first calibrated using APT response data by means of inverse analysis and later enhanced using laboratory results to apply to other conditions not included in the calibration. 
Thereafter, the loading and environment at NCAT were simulated and the advanced models applied to forecast measured resilient responses. Referring first to the anisotropic model, it was found that only mild improvements over the isotropic elastic case were offered and hence concluded that the added complexity involved in the anisotropic analysis did not prove worthy. As for the viscoelastic model, it was found that although computationally more demanding, a relatively simple calibration procedure could be followed, involving laboratory test results and one additional free parameter over the isotropic elastic case. The resulting calculations generated superior forecastability compared to the other two theories suggesting that this model should be preferred in the future. It is important to note that the related mathematical derivation was based on the elastic-viscoelastic correspondence principle, considerably simplifying the computational implementation, allowing readily available elastic programs to be utilized.

\subsection{RECCOMENDATIONS AND IMPLEMENTATION}

As previously stated, the main objective of this study was to try and link INDOT's APT results with those obtained at NCAT for nominally similar pavement systems. In the present work this objective was pursued by means of a mechanistic approach given that establishing a direct comparison was deemed unachievable. However, since both experiments were not a priori designed and carried out with a pure mechanistic interpretation in mind, the collected results limited the analysis efforts to dealing with resilient responses only, disregarding performance, i.e., permanent deformations (rutting) and load induced cracking. Henceforth, as a general recommendation, it is suggested that the traditional (empirical) approach of using APT studies as 'rut testers' or 'pavement comparators' be completely abandoned for future APT studies in favor of more modern (advanced) uses. The benefits gained from employing empirical/oversimplified approaches in the past are mostly exhausted, and if meaningful improvements are to be made in the pavement design field, future efforts should be placed on developing a more rational framework.

Subsequently, it is proposed that future APT research studies should aim at developing a mechanistic scheme for applying APT results to field conditions for 
similar constructions. Once such a methodology is available, huge financial benefits can be gained, for example by using the facility as a learning tool to improve pavement design methods or to promote the incorporation of new nontraditional materials. The present work offered a mechanistic approach to account for resilient (recoverable) responses. However, the proposed method was developed and validated using only one pavement type. As a first future step, this work should be reapplied, validated, and refined (if found necessary) using other pavement systems ${ }^{1}$. At a later stage, the analysis process should be extended to account for permanent (irrecoverable) deformations ${ }^{2}$ and to account for cracking of the HMA layers. It is further proposed that the research approach continue along the same lines as done here, i.e., building two nominally identical instrumented pavement systems, one in the INDOT APT and another in the field. The APT pavement should be thoroughly investigated to generate predictions for field conditions; these are then assessed/validated by comparison with field measurements.

It is important to emphasize that laboratory work, and advancement of our basic understanding of how the individual materials behave, plays an essential role here in that it is equally as important as the structural experiments for achieving the aforementioned objective. Only through the availability of both high quality structural data and high quality laboratory test results may it be possible to link construction processes, material properties and pavement behavior.

In what follows, and based on the study findings, a list of specific recommendations is offered for implementation in future studies involving INDOT's APT. The recommendations are separated into several topics: (i) embedded instrumentation; (ii) APT testing program; (iii) structural behavior; (iv) data acquisition; (v) laboratory work; and (vi) field project testing. Topics associated with new

\footnotetext{
${ }^{1}$ At the time of writing this report, a new research work was undertaken by INDOT and Purdue University (via the NCSC), involving the replication of a section of US31 in the APT facility. The pavement structure is composed of 17 in. (432 mm) HMA over a granular subgrade of which the top 16 in. $(406 \mathrm{~mm})$ were stabilized with cement.

${ }^{2}$ At the time of writing this report, a new research proposal was prepared, targeting the rutting behavior of pavements constructed with low void mixtures and involving the NCAT phase III test cycle. Naturally, this study would require addressing permanent deformations.
} 
construction of APT test sections (e.g., organization, scheduling, gauge installations, etc) are covered in Llenín and Pellinen (2004).

(i) Embedded Instrumentation. Before being installed, all gauge types should be checked for functionality, investigated for temperature sensitivity, and calibration factors validated. There is a need to devise ways to calibrate the gauges after installation in the pavement system given that their presence influences the free field behavior. Consider calibrating the pressure cells (used for vertical stresses) using single tire loading and comparing the 'volume' of stresses to the total applied load. There is a need to find more dependable and accurate methods to measure permanent stress and strain changes, and also moisture content changes. In addition to the gauge types installed in this study, it may be beneficial to add multi depth deflectometers. An attempt should be made to measure horizontal stresses in the different pavement layers (especially in the unbound layers) originating from the construction processes and later on due to trafficking. Additional stress and strain gauges should also be embedded deeper in the subgrade for better characterization and support for advanced modeling. A way to measure or estimate suction levels in the unbound materials should be sought.

(ii) APT Testing Program. Each experiment should be performed under at least three different temperature levels within the available range, e.g., $60^{\circ} \mathrm{F}\left(15.5^{\circ} \mathrm{C}\right), 77^{\circ} \mathrm{F}$ $\left(25.0^{\circ} \mathrm{C}\right)$, and $95^{\circ} \mathrm{F}\left(35^{\circ} \mathrm{C}\right)$. When switching from one temperature to another no loading should be applied until sufficient time (of about a week) has elapsed for the conditions to stabilize while monitoring and recording instrumentation readings; doing this will help to quantify the sensitivity of the instrumentation to temperature changes which could potentially be used later to adjust the raw readings. At any given temperature, APT passes should initially be applied using the super-single tire and later using the dual-tire assembly. For each temperature, the loadings should be executed at four different speeds within the available range, e.g., $0.05 \mathrm{mph}(0.08$ $\mathrm{km} / \mathrm{h}), 0.50 \mathrm{mph}(0.80 \mathrm{~km} / \mathrm{h}), 1.0 \mathrm{mph}(1.61 \mathrm{~km} / \mathrm{h})$, and $5.0 \mathrm{mph}(8.0 \mathrm{~km} / \mathrm{h})$. Also, at any given speed data should be collected at different load levels to study nonlinear response with respect to load level, e.g., 5 kips (2,270 kg); 7.5 kips (3,400 kg); 15.0 kips (6,800 kg) and 20 kips (9,070 kg). Modification of the APT should be considered 
so that two new loading modes can be applied to the surface of the pavement: horizontal (shear) and turning (torsion); these will allow the study of intersection conditions. An investigation to study the effects of various tire pressures should also be targeted. In this connection, a way to measure the actual loading area and distribution of stresses under the APT tires should be explored.

(iii) Structural Behavior. Completed structures in the APT should be tested using the Dynamic Cone Penetrometer, but also using more advanced geotechnical equipment such as the pressuremeter (cavity expansion). FWD testing should periodically be performed while recording the entire time history of the loading and deflections. At the same time, the responses captured by the embedded gauge array should also be recorded. Vertical surface deflection during APT testing and also during FWD testing ought to be monitored with external LVDTs. In addition, stand-alone accelerometers and geophones should be attached to the pavement surface to supplement the LVDTs. During all experiment types rutting measurements should be collected; more profiles ought to be collected at the initial stages of the experiment where most of the rutting is accumulated. The first profile measurement, taken before any passes are applied, should be repeated several times for each cross section since these are used as reference for all other measurements and hence need to be determined at a higher accuracy level. A method should be found for measuring surface profiles such that the loading system does not have to stop; this will facilitate the study of permanent deformation development. A systematic way to detect and record cracks as they appear (and when they appear) on the surface should be found; in this connection, a method should be developed to ascertain whether the observed cracks are so-called 'bottom up’ or 'top down'. Friction testing in the APT should be routinely performed, e.g., using the Circular Texture Meter (ASTM E-2157) and the Dynamic Friction Tester (ASTM E-1911). This type of data may be used to investigate the effects of temperature, wheel wander, tire type, and loading intensity on frictional attributes. With additional friction data from the corresponding field study, a scheme may be developed for using APT experiments to forecast friction performance.

(iv) Data Logging. All types of available data should continuously be recorded from the very instant of gauge installation, throughout all testing modes, until the test 
sections are removed. Data sampling rates should be varied based on the current 'action'; e.g., use 100 scans per second for APT passes (including when the wheels are lifted of the ground and returned to the startup location), use 5,000 scans per second for FWD tests, and record data every 5 to 10 minutes when monitoring environmental changes. The carriage position relative to the gauge array ought to be recorded at all time; this information is very important for performing inverse analysis. In the present study the APT carriage position was only recorded in the longitudinal direction. In future studies, whenever wander is applied, lateral positions of the carriage should also be recorded.

(v) Laboratory Work. Standard tests such as soil classification, laboratory compaction, HMA complex modulus, and resilient modulus for the unbound materials should be performed. An attempt should be made to employ a large range of conditions in these tests, e.g., execute the resilient modulus tests at different compaction and moisture levels. In addition, capabilities to perform more advanced tests should be developed; e.g., creep and recovery for the HMA in uniaxial (either tension or compression) or isotropic conditions. In this connection, radial strain measurements in all mechanical tests should be included. Whenever possible, test specimens should be fabricated from samples cut or cored from the as-built pavement.

(vi) Field project testing. As discussed in Topic (i) above, field project should be instrumented during construction by embedding gauges similar to the APT study or by retrofitting an existing pavement with gauges (e.g., temperature probes and multi depth deflectometers). Response data in the field may be collected only at certain times during which the lane will be closed to traffic and a truck of known weight and speed will drive over the gauge array. It is very important that the applied loading be accurately positioned in space $(x, y, z)$ and time $(t)$ relative to the gauges. For longitudinal positioning, a triggering device is suggested that can sense reflective targets mounted on the truck; for transverse positioning it may be sufficient to mark the pavement with lines spaced 1 to 2 in. (25 to $51 \mathrm{~mm}$ ) apart and take high definition video of the moving truck. Other tests should be conducted to investigate the overall structural behavior, as recommended in Topic (iii) above. 


\section{REFERENCES}

Al-Qadi, I. L. (2007), “True Viscoelastic Analyses of Pavement Structures to Validate \& Enhance Current Modulus Selection from Load Pulse Duration in NCHRP 1-37a Design Guide," obtained through private communication from the FHWA, TFHRC.

Andrei, D., Witczak, M. W., Schwartz, C. W., and Uzan, J. (2004), "Harmonized Resilient Modulus Test Method for Unbound Pavement Materials," Transportation Research Record 1874, Journal of the Transportation Research Board, pp. 29-37.

ARA Inc. (2004), "Guide for Mechanistic-Empirical Design of New and Rehabilitated Pavement Structures,” Applied Research Associates Inc., ERES Consultants Division, National Cooperative Highway Research Program (NCHRP) Project 1-37A, Final Report, Transportation Research Board, Washington, DC.

Austroads (2004), "Pavement Design - A Guide to the Structural Design of Road Pavements," Austroads Publication No. AP-G17/04, Sydney, Australia.

Barde, V. and Cardone, F. (2004), "Dynamic Modulus Testing of NCAT Mixtures," CE 597 Course Report, submitted to Dr. Terhi Pellinen, School of Civil Engineering, Purdue University.

Barden, L. (1963), "Stresses and Displacements in a Cross-anisotropic Soils," Géotechnique, Vol. 13 (3), pp. 198-210.

Brown, E. R., Cooley, L. A., Hanson, D., Lynn, C., Powell, B., Prowell, B., and Watson, D. (2002), "NCAT Test Track Design, Construction, and Performance," National Center for Asphalt Technology, Report No. 02-12.

Brown, S. F. (1977), "State-of-the-Art Report on Field Instrumentation for Pavement Experiments," Transportation Research Record 640, Journal of the Transportation Research Board, pp. 13-28.

Burmister, D. M. (1943), "The Theory of Stresses and Displacements in Layered Systems and Application to the Design of Airport Runways," Proceedings of the Highway Research Board, Vol. 23, Washington, D.C., pp. 126-148.

Burmister, D. M. (1945), "The General Theory of Stresses and Displacements in Layered Systems,” Journal of Applied Physics, Vol. 16, pp. 89-94 (Part I); pp. 126-127 (Part II); pp. 296-302 (Part III).

Christian, J. T. (1968), "Undrained Stress Distribution by Numerical Methods,” ASCE Journal of the Soil Mechanics and Foundation Engineering Division, Vol. 94 (6), pp. 1335-1345. 
De Jong, D. L., Peatz, M. G. F., and Korswagen, A. R. (1973), “Computer Program Bisar Layered Systems Under Normal and Tangential Loads,” Konin Klijke ShellLaboratorium, Amsterdam, External Report AMSR.0006.73.

Di Benedetto, H., Delaporte, B., and Sauzeat, C. (2007), “Three-Dimensional Linear Behavior of Bituminous Materials: Experiments and Modeling, ” International Journal of Geomechanics, Vol. 7(2), pp 149-157.

Duncan, J. M., Williams, G. W., Sehn, A. L., and Seed, R. B. (1991), "Estimation of Earth Pressures due to Compaction,” Journal of Geotechnical Engineering, Vol. 117 (12), pp. 1833-1847.

Dunnicliff, J. (1988), “Geotechnical Instrumentation for Monitoring Field Performance,” John Wiley and Sons, Inc., New York.

Elseifi, M. A., Al-Qadi, I. L., and Yoo, P. J. (2006), "Viscoelastic Modeling and Field Validation of Flexible Pavements,” Journal of Engineering Mechanics, Vol. 132 (2), pp. 172-178.

Freeman, R. B., Tommy, C. H, McEwen, T., and Powell, R. B. (2001), "Instrumentation at the National Center for Asphalt Technology Test Track," U.S. Army Corps of Engineers, Engineer Research and Development Center, Report No. ERDC TR-01-9.

Fylstra, D., Lasdon, L., Watson, J., and Waren, A. (1998), "Design and Use of the Microsoft Excel Solver,” Interfaces, Vol. 28(5), pp. 29-55.

Galal, K. A. and White, T. D. (1999), “INDOT-APT Test Facility Experience,” Paper CS8-4, presented at the International Conference on Accelerated Pavement Testing, Reno, Nevada, October 18-20.

Galal, K. A., White T. D., and Reck, C. (1998), “Accelerated Pavement Testing Facility,” INDOT - Division of Research report, West Lafayette, Indiana.

Graham, J., and Houlsby, G. T. (1983), “Anisotropic Elasticity of a Natural Clay,” Géotechnique, Vol. 33 (2), pp. 165-180.

Huang, H. (1995), "Analysis of Accelerated Pavement Tests and Finite Element Modeling of Rutting Phenomenon,” Ph.D. dissertation, Purdue university.

Huang, Y. H. (2004), "Pavement Analysis and Design,” Second Edition, Pearson Prentice Hall, p. 775.

Hufferd, W. L., and Lai, J. S. (1978), “Analysis of N-layered Viscoelastic Pavement Systems,” Report FHWA-RD-78-82, Federal Highway Administration, p. 22.

Labuz, J. F., and Theroux, B. (2005), “Laboratory Calibration of Earth Pressure Cells,” Geotechnical Testing Journal, Vol. 28 (2). 
Lakes, R. S. (1998), Viscoelastic Solids, CRC Press (Boca Raton, FL), p. 476.

Lekhnitskii, S. G. (1963), “Theory of Elasticity of an Anisotropic Elastic Body, HoldenDay, San Francisco, p. 404.

Levenberg, E. (2006), "Constitutive Modeling of Asphalt-Aggregate Mixes with Damage and Healing,” Ph.D. Dissertation, Technion - Israel Institute of Technology.

Levenberg, E., and Shah, A. (2008), "Interpretation of Complex Modulus Test Results for Asphalt-Aggregate Mixes,” ASTM Journal of Testing and Evaluation, Vol. 36 (4).

Llenín, J. A., and Pellinen, T. K. (2004), "Validation of NCAT Structural Test Track Experiment using INDOT APT Facility," Interim Draft Final Report, Joint Transportation Research Program, SPR 2813 Project, Purdue University.

Llenín, J. A., Pellinen, T. K., and Abraham, D. M. (2006), "Construction Management of a Small-Scale Accelerated Pavement Testing Facility," Journal of Performance of Constructed Facilities, Vol. 20 (3), pp. 229-236.

Locket, F. J. (1972), Nonlinear Viscoelastic Solids, Academic Press Inc. (London), p. 195.

Love, A. E. H. (1923), “Treatise on the Mathematical Theory of Elasticity”, Cambridge University Press, UK.

Lytton, R. L., Uzan, J., Fernando, E. G., Roque, R., Hiltunen, D., and Stoffels, S. M. (1993), "Development and Validation of Performance Prediction Models and Specifications for Asphalt Binders and Paving Mixes,” Report SHRP A-357, Strategic Highway Research Program, National Research Council, Washington, D.C.

Monfore, G. E. (1950), "An Analysis of the Stress Distribution in and near Stress Gauges Embedded in Elastic Soils,” Structural Laboratory Report No. SP 26, U.S. Bureau of Reclamation, Denver, CO.

NCHRP (2002), "Standard Test Method for Dynamic Modulus of Asphalt Concrete Mixtures”, Provisional Test Method DM-1, Project 1-37A, Arizona State University.

Oda, M., Nemat-Nasser, S., and Konish, J. (1985), "Stress-induced Anisotropy in Granular Masses,” Journal of Soils and Foundation, Vol. 25 (3), pp. 85-97.

Peattie, K. R., and Sparrow, R. W. (1954), “The Fundamental Action of Earth Pressure Cells,” Journal of the Mechanics and Physics of Solid, Vol. 2, pp. 141-155.

Pipkin, A. C. (1972), Lectures on Viscoelasticity Theory, Applied Mathematical Sciences Vol. 7, Springer-Verlag Inc. (New York), pp. 180.

Plazek, D. J. (1996), “Oh, Thermorheological Simplicity, Wherefore Art Thou?,” 1995 Bingham Medal Address, Journal of Rheology, Vol. 40 (6), pp. 987-1014. 
Poulos, H. G., and Davis, E. H. (1974), "Elastic Solutions for Soil and Rock Mechanics, Center for Geotechnical Research,” John Wiley \& Sons, Inc. (reprinted and corrected 1991).

Powell, R. B., and Brown, E. R. (2004), "Construction of the 2003 NCAT Pavement Test Track,” National Center for Asphalt Technology, Draft Report.

Priest, A. L., and Timm, D. H. (2006), "Methodology and Calibration of Fatigue Transfer Functions for Mechanistic-Empirical Flexible Pavement Design,” National Center for Asphalt Technology, Report 06-03.

Priest, A. L., Timm, D. H., and Barrett, W. E. (2005), "Mechanistic Comparison of Wide-base Single vs. Standard Dual Tire Configurations,” National Center for Asphalt Technology, Report 05-03.

Saadeh, S., Tashman, L., Masad, E., and Mogawer, W. (2002), "Spatial and Directional Distribution of Aggregates in Asphalt Mixes," ASTM Journal of Testing and Evaluation, Vol. 30 (6).

Schapery, R. A. (1962), “Approximate Methods of Transform Inversion for Viscoelastic Stress Analysis,” Proceedings of the $4^{\text {th }}$ U.S. National Congress on Applied Mechanics, Vol. 2, ASME, pp. 1075-1085.

Schapery, R. A. (1965), “A Method of Viscoelastic Stress Analysis using Elastic Solutions,” Journal of the Franklin Institute, Vol. 279 (4), pp. 268-289.

Schapery, R. A. (1974), "Viscoelastic Behavior and Analysis of Composite Materials, ” Mechanics of Composite Materials, Vol. 2, pp. 85-168, edited by G. P. Sendeckyj, Academic Press (New York).

Schwarzl, F., and Staverman, A. J. (1952), “Time-Temperature Dependence of Linear Viscoelastic Behavior,” Journal of Applied Physics, Vol. 23 (8), pp. 838-843.

Shields, D. H., Zeng, M., and Kwok, R. (1998), "Nonlinear Viscoelastic Behavior of Asphalt Concrete in Stress Relaxation," Journal of the Association of Asphalt Pavement Technologists, Vol. 67.

Singh, S. J. (1986), "Static Deformation of a Transversely Isotropic Multilayered Halfspace by Surface Loads," Physics of the Earth and Planetary Interiors, Vol. 42 (4), pp. 263-273.

Sugihara, M. (1987), "Methods of Numerical Integration of Oscillatory Functions by the DE-formula with Richardson Extrapolation,” Journal of Computational and Applied Mathematics, Vol. 17, pp. 47-68.

Tabatabaee, N., and Sebaaly, P. (1990), "State-of-the-Art Pavement Instrumentation,” Transportation Research Record 1260, Journal of the Transportation Research Board, pp. 246-255. 
Taylor, D. W., 1945, "Review of Pressure Distribution Theories, Earth Pressure Cell Investigations, and Pressure Distribution Data," Contract Report W22-053 eng-185, U.S. Army Engineer Waterways Experiment Station, Vicksburg, MS.

Tesarik, D. R., Seymour, J. B., Williams, T. J., Martin, L. A., and Jones, F. M. (2006), "Temperature Corrections to Earth Pressure Cells Embedded in Cemented Backfill," Report of Investigations No. 9665, U.S. Department of Health and Human Services, Centers for Disease Control and Prevention, National Institute for Occupational Safety and Health, Spokane Research Laboratory, Spokane, WA.

Theroux, B., Labuz, J. F., and Dai, S. (2001), "Field Installation of an Earth Pressure Cell,” Transportation Research Record 1772, Journal of the Transportation Research Board, pp. 12-19.

Timm, D. H., and Priest, A. L. (2006), "Material Properties of the 2003 NCAT Test Track Structural Study,” National Center for Asphalt Technology, Report No. 06-01.

Timm, D. H., Priest, A. L., and McEwen, T. V. (2004), "Design and Instrumentation of the Structural Pavement Experiment at the NCAT Test Track," National Center for Asphalt Technology, Report No. 04-01.

Timm, D. H., West, R. C., Priest, A. L., Powell, B., Selvaraj, I., Zhang, J., and Brown, E. R. (2006), "Phase II NCAT Test Track Results," National Center for Asphalt Technology, Report 06-05.

Tory, A. C., and Sparrow, R. W. (1967), "The Influence of Diaphragm Flexibility on the Performance of an Earth Pressure Cell,” Journal of Scientific Instruments, Vol. 44, pp. 781-785.

Tutumluer, E., and Thompson, M. R. (1997), “Anisotropic Modeling of Granular Bases in Flexible Pavements," Transportation Research Record 1557, Journal of the Transportation Research Board, pp. 18-26.

Uzan, J. (1976), "The Influence of the Interface Condition on the Stress Distribution in a Layered System,” Transportation Research Record 616, Journal of the Transportation Research Board, pp. 71-73.

Uzan, J. (1985), “Characterization of Granular Material,” Transportation Research Record 1022, Journal of the Transportation Research Board, pp. 52-59.

Uzan J. (1992), "Resilient Characterization of Pavement Materials," International Journal of Numerical and Analytical Methods in Geomechanics, Vol. 16, pp. 453-459.

Uzan, J., and Levenberg, E. (2007), “Advanced Testing and Characterization of Asphalt Concrete Materials in Tension,” International Journal of Geomechanics, Vol. 7 (2), pp. 158-165. 
von Karman, T., and Biot, M. A. (1940), Mathematical Methods in Engineering: An Introduction to the Mathematical Treatment of Engineering Problems, McGraw-Hill Inc., New York, p. 505.

Weiler, W. A., and Kulhawy, F. H. (1982), "Factors Affecting Stress Cell Measurements in Soil,” ASCE Journal of the Geotechnical and Foundation Division, Vol. 108 (GT12), pp. 1529-1548.

White, T. D., Albers, J. M., and Haddock, J. E. (1990), “An Accelerated Testing System to Determine Percent Crushed Aggregate Requirements in Bituminous Mixtures - Final Report," Joint Transportation Research Program, FHWA/IN/JTRP-90-8, Purdue University.

Williams, M. L., Landel, R. F., and Ferry, J. D. (1955), “The Temperature Dependence of Relaxation Mechanisms in Amorphous Polymers and Other Glass-forming Liquids,” Journal of the American Chemical Society, Vol. 77, pp. 3701-3707.

Witczak, M. W., and Uzan, J. (1988), “The Universal Airport Design System, Report I of IV: Granular Material Characterization,” Department of Civil Engineering, University of Maryland, College Park.

Wolf, K. (1935), "Distribution of Stress in a Half-plane and a Half-space of Anisotropic Material, “ ZAMM - Journal of Applied Mathematics and Mechanics, Vol. 15 (5), pp. 249-254. 


\section{APPENDICES (DVD UPON REQUEST)}

'Appendix_A', 'Appendix_B' and 'Appendix_C' are available on a DVD from the JTRP Office upon request by e-mail jtrp@ecn.purdue.edu or call 765-494-9310. Each folder contains the associated appendix material. A short description of each is hereafter provided.

\section{APPENDIX A: RAW EXPERIMENTAL DATA}

The 'Appendix_A' folder on the DVD contains the raw experimental data collected during this entire study. Three subfolders are included, named: 'A1_APT Experiment', 'A2_Common Data', and 'A3_NCAT Experiment'.

The A1 Subfolder contains information related to the APT study. It consists of the following six folders: (i) A11_ RTR Files. These are text files that include information recorded by the PC running the APT carriage. An explanation on how to read these RTR files can be found in the PDF document placed in the same folder; (ii) A12_Moisture Data. These files contain the recorded moisture data. A viewer program is needed (also provided); (iii) A13_StrainSmart Data. These files contain the stress, strain and temperature data. The write protected program 'StrainSmart' is required to read these files (not included); (iv) A14_Pictures. Includes the pictures and video clips taken during the APT experiment; (v) A15_Rutting Profiles. All rutting measurements done in the APT are included, sorted according to measurement date. Indexes 1 and 2 in the rutting file names refer to sections $\mathrm{n} 1$ and $\mathrm{n} 2$ before rehabilitation (respectively) while indexes 3 and 4 refer to test sections $\mathrm{n} 1$ and $\mathrm{n} 2$ after rehabilitation (respectively); and (vi) A16_FWD. Includes FWD files that consist of peak deflection data (and corresponding HMA temperatures.

The A2 Subfolder contains information that is common to both the APT and NCAT experiments. This subfolder consists of the following three folders, having self explanatory names: (i) A21_Beam Fatigue; (ii) A22_Complex Modulus; and (iii) A23_Presentations.

The A3 Subfolder contains information related to the NCAT study. It consists of nine folders (again, self explanatory names): (i) A31_Pictures; (ii) A32_DCPT\&CBR; 
(iii) A33_FWD; (iv) A34_Reports\&Presentations; (v) A35_HMA; (vi) A36_Resilient Modulus; (vii) A37_Response\&Performance; (viii) A38_Spray Applications; and (ix) A39_Design\&Spec.

\section{APPENDIX B: COMPUTER PROGRAMS}

The 'Appendix_B' folder on the DVD contains two structural analysis programs developed under this study: (i) 'ELLEA1' which is based on isotropic LET (see Chapter 4); and (ii) 'ELLEA2' which is based on anisotropic LET (see Chapter 5). These programs are built into Excel workbooks for real-time computations and ease of use. Two versions are included for each program: (i) an Excel 2003 version identified by a file name extension .xls; and (ii) an Excel 2007 version identified by a file name extension .xlsx.

In order for these programs to run correctly it is important to enable the Analysis ToolPak, an Add-In normally included in Excel, which sometimes needs manual installation (a one time event). It is important to note that both structural analysis programs do not contain any VBA code so that any Macro related virus warning can be ignored. If you experience any trouble or have any additional questions or requests, contact me through this Email address: Eyal.Levenberg@yahoo.com.

\section{APPENDIX C: REPORTS AND CORRESPONDANCE}

The 'Appendix_C' folder on the DVD contains previous reports and correspondence related to the APT project (PDF format). These are chronologically ordered, each placed in a separate folder. This report is also included so that it can be reviewed or reproduced with colors if desired. Note that some of the PDF files are scanned versions of the printed originals; as such they are relatively large in size.

The included titles are as follows: (i) C1_Research Proposal (Pellinen and Galal, May 2003); (ii) C2_ Interim Instrumentation Plan Report (Llenín, April 2004); (iii) C3_Interim Materials Shipping Plan Report (Llenín, April 2004); (iv) C4_APT Construction Specifications (Llenín and Pellinen, May_2004); (v) C5_Instrumentation Installation Procedure Report (Llenín, May 2004); (vi) C6_APT First Construction Cycle Report (Llenín and Pellinen, August 2004); (vii) C7_Feasibility Analysis (Llenín 
and Pellinen, November 2004); (viii) C8_Dynamic Modulus Testing of NCAT Mixes (Barde and Cardone, December 2004); (ix) C9_Interim Draft Final Report (Llenín and Pellinen, December 2004); (x) C10_APT Instrumentation and Loading Experiments (Pellinen and Webster, January 2005); (xi) C11_Preparation of Beams for SPR-2813 (Pellinen, Webster and Brower, February 2005); (xii) C12_Advanced Analysis of Beam Fatigue Test Results of NCAT mixes (Agrawal, August 2005); (xiii) C13_Laboratory Fatige Testing of HMA (Webster and Pellinen, May 2005); (xiv) C14_Quarterly Progress Report 2005 (Pellinen and Nantung, December 2005); and (xv) C15_This (Final) Report (Levenberg, 2008). 